\title{
Preliminary Metallogenic Belt and Mineral Deposit Maps for Northeast Asia
}

By Sergey M. Rodionov ${ }^{1}$, Alexander A. Obolenskiy ${ }^{2}$, Elimir G. Distanov ${ }^{2}$, Gombosuren Badarch ${ }^{3}$, Gunchin Dejidmaa ${ }^{4}$, Duk Hwan Hwang $^{5}$, Alexander I.Khanchuk ${ }^{6}$, Masatsugu Ogasawara ${ }^{7}$, Warren J. Nokleberg ${ }^{8}$, Leonid M. Parfenov ${ }^{9}$, Andrei V. Prokopiev ${ }^{9}$, Zhan V. Seminskiy ${ }^{10}$, Alexander P. Smelov ${ }^{9}$, Hongquan Yan ${ }^{11}$, Gennandiy V. Birul'kin ${ }^{9}$, Yuriy V. V. Davydov ${ }^{9}$, Valeriy Yu. Fridovskiy ${ }^{12}$, Gennandiy N. Gamyanin ${ }^{9}$, Ochir Gerel' ${ }^{13}$, Alexei V. Kostin ${ }^{9}$, Sergey A. Letunov ${ }^{14}$, Xujun $\mathrm{Li}^{11}$, Valeriy M. Nikitin ${ }^{12}$, Sadahisa Sudo ${ }^{7}$ Vitaly I. Sotnikov ${ }^{2}$, Alexander V. Spiridonov ${ }^{14}$, Vitaly A. Stepanov ${ }^{15}$, Fengyue Sun ${ }^{11}$, Jiapeng Sun ${ }^{11}$, Weizhi Sun ${ }^{11}$, Valeriy M. Supletsov ${ }^{9}$, Vladimir F. Timofeev ${ }^{9}$, Oleg A. Tyan ${ }^{9}$, Valeriy G. Vetluzhskikh ${ }^{9}$, Koji Wakita ${ }^{7}$, Yakov V. Yakovlev ${ }^{9}$, and Lydia M. Zorina ${ }^{14}$

\section{Open-File Report 03-204}

\section{3}

This report is preliminary and has not been reviewed for conformity with U.S. Geological Survey editorial standards or with the North American Stratigraphic Code. Any use of trade, firm, or product names in this publication is for descriptive purposes only and does not imply endorsement by the U.S. Government.

${ }^{1}$ Russian Academy of Sciences, Khabarovsk

${ }^{2}$ Russian Academy of Sciences, Novosibirsk

${ }^{3}$ Mongolian Academy of Sciences, Ulaanbaatar

${ }^{4}$ Mineral Resources Authority of Mongolia, Ulaanbaatar

${ }^{5}$ Korean Institute of Geology, Mining, and Materials, Taejon

${ }^{6}$ Russian Academy of Sciences, Vladivostok

${ }^{7}$ Geological Survey of Japan/AIST, Tsukuba

${ }^{8}$ U.S. Geological Survey, Menlo Park

${ }^{9}$ Russian Academy of Sciences, Yakutsk

${ }^{10}$ Irkutsk State Technical University, Irkutsk

${ }^{11}$ Jilin University, Changchun

${ }^{12}$ Yakutian State University, Yakutsk

${ }^{13}$ Mongolian University of Science and Technology, Ulaanbaatar

${ }^{14}$ Russian Academy of Sciences, Irkutsk

${ }^{15}$ Russian Academy of Sciences, Blagoveschensk 
Prepared in Collaboration with Russian Academy of Sciences, Mongolian Academy of Sciences, Jilin University (Changchun Branch), Korean Institute of Geology, Mining, and Materials, and Geological Survey of Japan/AIST.

\section{U.S. DEPARTMENT OF THE INTERIOR U.S. GEOLOGICAL SURVEY}

\section{Introduction and Companion Studies}

The metallogenic belts and locations of major mineral deposits of Northeast Asia are portrayed on Sheets 1-4. Sheet 1 portrays the location of significant lode deposits and placer districts at a scale of $1: 7,500,000$. Sheets $2-4$ portray the metallogenic belts of the region in a series of 12 time-slices from the Archean through the Quaternary at a scale of $1: 15,000,000$. For all four map sheets, a generalized geodynamics base map, derived from a more detailed map by Parfenov and others (2003), is used as an underlay for the metallogenic belt maps. This geodynamics map underlay permits depicts the major host geologic units and structures that host metallogenic belts. Four tables are included in this report. A hierarchial ranking of mineral deposit models is listed in Table 1. And summary features of lode deposits, placer districts, and metallogenic belts are described in Tables 2, 3, and 4, respectively.

The metallogenic belts for Northeast Asia are synthesized, compiled, described, and interpreted with the use of modern concepts of plate tectonics, analysis of terranes and overlap assemblages, and synthesis of mineral deposit models. The data supporting the compilation are: (1) comprehensive descriptions of mineral deposits; (2) compilation and synthesis of a regional geodynamics map the region at 5 million scale with detailed explanations and cited references; and (3) compilation and synthesis of metallogenic belt maps at 15 million scale with detailed explanations and cited references. These studies are part of a major international collaborative study of the Mineral Resources, Metallogenesis, and Tectonics of Northeast Asia that is being conducted from 1997 through 2002 by geologists from earth science agencies and universities in Russia, Mongolia, Northeastern China, South Korea, Japan, and the USA. Companion studies and previous publications are: (1) a detailed geodynamics map of Northeast Asia (Parfenov and 2003); (2) a compilation of major mineral deposit models (Rodionov and Nokleberg, 2000; Rodionov and others, 2000; Obolenskiy and others, 2003); and (3) a database on significant metalliferous and selected nonmetalliferous lode deposits, and selected placer districts (Ariunbileg and others, 2003).

\section{Concepts and Problems for Synthesis of Metallogenic Belts}

Metallogenic belts are characterized by a narrow age of formation, and include districts, deposits, and occurrences. The metallogenic belts are synthesized and described for the main structural units of the North Asian Craton and Sino-Korean Craton, framing orogenic belts that consist of collage of accreted tectonostratigraphic terranes, younger overlap volcanic and sedimentary rock sequences, and younger stitching plutonic sequences. The major units in the region are the North Asian Craton, exterior passive continental margin units (Baikal-Patom, Enisey Ridge, Southern Taymir, and Verkhoyansk passive continental margin units), the early Paleozoic Central Asian orogenic belt, and various Mesozoic and Cenozoic continental margin arcs. Metallogenic belts are interpreted according to specific geodynamic environments including cratonal, active and passive continental margin, continentalmargin arc, island arc, oceanic or continental rift, collisional, transform-continental margin, and impact.

Previous metallogenic units published by various authors for studies of metallogenic zonation include (Bilibin, 1955; Itsikson and others, 1965; Shatalov, 1965; Itsikson, 1973, 1979; Guild, 1978; Scheglov, 1980; Mitchell and Garson, 1981; Radkevich, 1982; Tomson, 1988; Zonenshain and others, 1992; Koroteev, 1996; Parfenov and others, 1999; Sukhov and others, 2000; Plyuschev, 2001): (1) planetary deposit-hosting province or planetary metallogenic belt $\left(\geq 1000\right.$ by $10^{3}$ $\left.\mathrm{km}^{2}\right)$; (2) deposit-hosting belt or metallogenic belt (150 to 1000 by $10^{3} \mathrm{~km}^{2}$ ); (3) deposit-hosting system or metallogenic system (40 to 150 by $10^{3} \mathrm{~km}^{2}$ ); (4) deposit-hosting zone or metallogenic zone ( 20 to 40 by $10^{3} \mathrm{~km}^{2}$ ); (5) deposit-hosting subzone or metallogenic subzone ( 2 to 20 by $10^{3} \mathrm{~km}^{2}$ ); and (6) ore district ( 0.4 to 2.0 by $\left.10^{3} \mathrm{~km}^{2}\right)$.

However, often determination of differences between some of these metallogenic units is difficult. Examples are metallogenic system versus metallogenic zone, or ore district versus deposit-hosting subzone. For this study, only a two simple terms are employed: metallogenic belt and contained district. Generally, the size of metallogenic belts is partly a function of the scale of the analysis. For this study, metallogenic belts are synthesized and compiled at $5 \mathrm{M}$ scale. 
In this study, a metallogenic belt is essentially the synonymous with the term mineral resource tract as originally defined by Pratt (1981) and used for assessment of mineral resource potential in the USA, as in exemplified in Luddington and Cox (1996). The metallogenic belt maps and underlying regional geologic (terrane and overlap assemblage maps) constitute a basic part of the three-part methodology of quantitative mineral resource assessment as described by Cox (1993) and Singer $(1993,1994)$.

The following concepts are employed for the synthesis of metallogenic belts.

Mineral Deposit Association. Each mineral resource tract (or metallogenic belt) includes a single mineral deposit type or a group of coeval, closely-located and genetically-related mineral deposits types.

Geodynamic Event for Deposit Formation. Each metallogenic belt contains a group of coeval and genetically related deposits that were formed in a specific geodynamic event. Examples are collision, continental-margin arc, accretion, rifting, and others.

Favorable Geological Environment. Each metallogenic belt is underlain by a geological host rock and (or) structure that is favorable for a particular suite of mineral deposit types.

Tectonic or Geological Boundaries. Each mineral resource tract (or metallogenic belt) is usually bounded by favorable either stratigraphic or magmatic units, or by major faults (sutures) along which substantial translations have occurred.

Relation of Features of Metallogenic Belt to Host Unit. The name, boundaries, and inner composition of each metallogenic belt corresponds to previously define characteristics of rocks or structures hosting the deposits, and to a suite of characteristics for the group of deposits and host rocks.

With these definitions and principles, the area defined for a metallogenic belt is predictive or prognostic for undiscovered deposits. Consequently, the synthesis and compilation of metallogenic belts is a powerful tool for mineral exploration, land-use planning, and environmental studies.

For modern metallogenic analysis, three interrelated problems exist.

(1) What is the relation of geodynamics to regional or global metallogeny? As discussed by Zonenshain and others (1992) and Dobretsov and Kirdyashkin (1994), this problem includes the role of convective processes in mantle and mantle plumes, the global processes of formation of the continents and oceans, the dynamics of development of major tectonic units of the earth's crust, metallogenic evolution of the earth, and the role mantle processes in the origin of major-belts of deposits.

(2) What is relation of regional metallogeny to individual lithosphere blocks? As discussed by Guild (1978), Mitchell and Garson (1981), and Koroteev (1996), this problem includes the genesis of specific metallogenic belts as a function of specific geodynamic environments using the modem concepts of plate tectonics.

And (3) what is the relation of metallogeny to individual tectonostratigraphic terranes and overlap assemblages? As discussed by Nokleberg and others (1993, 1998) and Parfenov and others (1999), this problem includes the genesis of specific metallogenic belts in individual fault-bounded units of distinctive stratigraphy, defined as tectonostratigraphic terranes, and in younger overlapping assemblages often containing igneous rocks formed in continental margin or island arcs, along rift systems in continents, or along transform continental margins.

\section{Methodology of Metallogenic Analysis, Key Definitions, Geologic Time Scale, and Time Spans}

\section{Methodology of Metallogenic and Tectonic Analysis}

The compilation, synthesis, description, and interpretation of metallogenic belts of Northeast Asia is part of a intricate process to analyze the complex metallogenic and tectonic history of the region. The methodology for this type of analysis of consists of the following steps. (1) The major lode deposits are described and classified according to defined mineral deposit models. (2) Metallogenic belts are delineated. (3) Tectonic environments for the cratons, craton margins, orogenic collages of terranes, overlap assemblages, and contained metallogenic belts are assigned from regional compilation and synthesis of stratigraphic, structural, metamorphic, isotopic, faunal, and provenance data. The tectonic environments include cratonal, passive continental margin, metamorphosed continental margin, continental-margin arc, island arc, transform continental-margin arc, oceanic crust, seamount, ophiolite, accretionary wedge, subduction zone, turbidite basin, and metamorphic. (4) Correlations are made between terranes, fragments of overlap assemblages, and fragments of contained metallogenic belts. (5) Coeval terranes and their contained metallogenic belts are grouped into a single 
metallogenic and tectonic origin, for instance, a single island arc or subduction zone. (6) Igneous-arc and subduction-zone terranes, which are interpreted as being tectonically linked, and their contained metallogenic belts, are grouped into coeval, curvilinear arc-subduction-zone-complexes. (7) By use of geologic, faunal, and paleomagnetic data, the original positions of terranes and their metallogenic belts are interpreted. (8) The paths of tectonic migration of terranes and contained metallogenic belts are constructed. (9) The timings and nature of accretions of terranes and contained metallogenic belts are determined from geologic, age, and structural data; (10) The nature of collision-related geologic units and their contained metallogenic belts are determined from geologic data. And (11) the nature and timing of postaccretionary overlap assemblages and contained metallogenic belts are determined from geologic and age data.

\section{Key Metallogenic and Tectonic Definitions}

For the compilation, synthesis, description, and interpretation of metallogenic belts, the following and mineral deposit, metallogenic, and tectonic definitions are employed. The definitions are adapted from Coney and others (1980), Jones and others (1983), Howell and others (1985), Monger and Berg (1987), Nokleberg and others (1994a, b, 2001), Wheeler and others (1988), and Scotese and others (2001).

Accretion. Tectonic juxtaposition of two or more terranes, or tectonic juxtaposition of terranes to a craton margin. Accretion of terranes to one another or to a craton margin also defines a major change in the tectonic evolution of terranes and craton margins.

Accretionary wedge and subduction-zone terrane. Fragment of a mildly to intensely deformed complex consisting of varying amounts of turbidite deposits, continental-margin rocks, oceanic crust and overlying units, and oceanic mantle. Divided into units composed predominantly of turbidite deposits or predominantly of oceanic rocks. Units are interpreted to have formed during tectonic juxtaposition in a zone of major thrusting of one lithosphere plate beneath another, generally in zones of thrusting along the margin of a continent or an island arc. May include large faultbounded units with a coherent stratigraphy. Many subduction-zone terranes contain fragments of oceanic crust and associated rocks that exhibit a complex structural history, occur in a major thrust zone, and possess blueschist-facies metamorphism.

Collage of terranes. Groups of tectonostratigraphic terranes, generally in oceanic areas, for which insufficient data exist to separate units.
Craton. Chiefly regionally metamorphosed and deformed shield assemblages of Archean and Early Proterozoic sedimentary, volcanic, and plutonic rocks, and overlying platform successions of Late Proterozoic, Paleozoic, and local Mesozoic and Cenozoic sedimentary and lesser volcanic rocks.

Craton margin. Chiefly Late Proterozoic through Jurassic sedimentary rocks deposited on a continental shelf or slope. Consists mainly of platform successions. Locally has, or may have had an Archean and Early Proterozoic cratonal basement.

\section{Cratonal terrane. Fragment of a craton.}

Continental-margin arc terrane. Fragment of an igneous belt of coeval plutonic and volcanic rocks, and associated sedimentary rocks that formed above a subduction zone dipping beneath a continent. Inferred to possess a sialic basement.

Deposit. A general term for any lode or placer mineral occurrence, mineral deposit, prospect, and (or) mine.

Island-arc terrane. Fragment of an igneous belt of plutonic rocks, coeval volcanic rocks, and associated sedimentary rocks that formed above an oceanic subduction zone. Inferred to possess a simatic basement.

Metallogenic belt. A geologic unit (area) that either contains or is favorable for a group of coeval and genetically-related, significant lode and placer deposit models. With this definition, a metallogenic belt is a predictive for undiscovered deposits.

Metamorphic terrane. Fragment of a highly metamorphosed or deformed assemblage of sedimentary, volcanic, or plutonic rocks that cannot be assigned to a single tectonic environment because the original stratigraphy and structure are obscured. Includes intensely-deformed structural melanges that contain intensely-deformed fragments of two or more terranes.

Metamorphosed continental margin terrane. Fragment of a passive continental margin, in places moderately to highly metamorphosed and deformed, that cannot be linked with certainty to the nearby craton margin. May be derived either from a nearby craton margin or from a distant site.

Mine. A site where valuable minerals have been extracted.

Mineral deposit. A site where concentrations of potentially valuable minerals for which grade and tonnage estimates have been made. 
Mineral occurrence. A site of potentially valuable minerals on which no visible exploration has occurred, or for which no grade and tonnage estimates have been made.

Oceanic crust, seamount, and ophiolite terrane. Fragment of part or all of a suite of eugeoclinal deepmarine sedimentary rocks, pillow basalt, gabbro, and ultramafic rocks that are interpreted as oceanic sedimentary and volcanic rocks and the upper mantle. Includes both inferred offshore oceanic and marginal ocean basin rocks, minor volcaniclastic rocks of magmatic arc derivation, and major marine volcanic accumulations formed at a hotspot, fracture zone, or spreading axis.

Overlap assemblage. A postaccretion unit of sedimentary or igneous rocks deposited on, or intruded into, two or more adjacent terranes. The sedimentary and volcanic parts either depositionally overlie, or are interpreted to have originally depositionally overlain, two or more adjacent terranes, or terranes and the craton margin. Overlapping plutonic rocks, which may be coeval and genetically related to overlap volcanic rocks, link or stitch together adjacent terranes, or a terrane and a craton margin.

Passive continental margin terrane. Fragment of a craton margin.

Post-accretion rock unit. Suite of sedimentary, volcanic, or plutonic rocks that formed in the late history of a terrane, after accretion. May occur also on adjacent terranes or on the craton margin either as an overlap assemblage or as a basinal deposit. A relativetime term denoting rocks formed after tectonic juxtaposition of one terrane to an adjacent terrane.

Pre-accretion rock unit. Suite of sedimentary, volcanic, or plutonic rocks that formed in the early history of a terrane, before accretion. Constitutes the stratigraphy and igneous geology inherent to a terrane. A relative-time term denoting rocks formed before tectonic juxtaposition of one terrane to an adjacent terrane.

Prospect. A site of potentially valuable minerals in which excavation has occurred.

Significant mineral deposit. A mine, mineral deposit, prospect, or occurrence that is judged as important for the metallogenesis of a geographic region.

Subterrane. A fault-bounded unit within a terrane that exhibit similar, but not identical geologic history relative to another fault bounded unit in the same terrane.
Superterrane. An aggregate of terranes that is interpreted to share either a similar stratigraphic kindred or affinity, or a common geologic history after accretion (Moore, 1992). An approximate synonym is composite terrane.

Tectonic linkage. The interpreted association of a suite of coeval tectonic units that formed in the same region and as the result of the same tectonic processes. An example is the linking of a coeval continentalmargin arc, forearc deposits, a back-arc rift assemblage, and a subduction-zone complex, all related to the underthrusting of a continental margin by oceanic crust.

Tectonostratigraphic terrane. A fault-bounded geologic entity or fragment that is characterized by a distinctive geologic history that differs markedly from that of adjacent terranes (Jones and others, 1983; Howell and others, 1985).

Transform continental-margin arc. An igneous belt of coeval plutonic and volcanic rocks, and associated sedimentary rocks that formed along a transform fault that occurs along the margin of a craton, passive continental margin, and (or) collage of terranes accreted to a continental margin.

Turbidite basin terrane. Fragment of a basin filled with deep-marine clastic deposits in either an orogenic forearc or backarc setting. May include continentalslope and continental-rise turbidite deposits, and submarine-fan turbidite deposits deposited on oceanic crust. May include minor epiclastic and volcaniclastic deposits.

\section{Geologic Time Scale and Time Spans}

Geologic time scale units are according to the IUGS Global Stratigraphic Chart (Remane, 1998). For this study, for some descriptions of metallogenic belt and geologic units, the term Riphean is used for the Mesoproterozoic through Middle Neoproterozoic (1600 to $650 \mathrm{Ma}$ ), and the term Vendian is used for Neoproterozoic III (650 to $540 \mathrm{Ma}$ ).

According to the main geodynamic events and the major deposit-forming and metallogenic belt-forming events for Northeast Asia, the following twelve time spans are used for groupings of metallogenic belts.

Archean (>2500 Ma)

Paleoproterozoic (2500 to $1600 \mathrm{Ma}$ )

Mesoproterozoic (1600 to $1000 \mathrm{Ma}$ )

Neoproterozoic (1000 to $540 \mathrm{Ma}$ )

Cambrian through Silurian (540 to $410 \mathrm{Ma}$ )

Devonian through Early Carboniferous (Mississippian)

(410 to $320 \mathrm{Ma}$ ) 
Late Carboniferous (Pennsylvanian) through Middle Triassic (320 to $230 \mathrm{Ma}$ )

Late Triassic through Early Jurassic (230 to $175 \mathrm{Ma}$ )

Middle Jurassic through Early Cretaceous (175 to 96 $\mathrm{Ma})$

Cenomanian through Campanian (96 to $72 \mathrm{Ma}$ )

Maastrichnian through Oligocene (72 to $24 \mathrm{Ma}$ )

Miocene through Quaternary (24 to $0 \mathrm{Ma}$ )

\section{Mineral Deposit Models}

For descriptions of metallogenic belts, lode mineral deposits are classified into various models or types. Detailed descriptions are provided in the companion paper by Obolenskiy and others (2003). The following three main principles are employed for synthesis of mineral deposit models for this study. (1) Deposit forming processes are close related to rock forming processes (Obruchev, 1928) and mineral deposits originate as the result of mineral mass differentiation under their constant circulation in sedimentary, magmatic, and metamorphic circles of formation of rocks and geological structures (Smirnov, 1969). (2) The classification must be as more comfortable and understandable for appropriate user as possible. And (3) the classification must be open so that new types of the deposits can be added in the future (Cox and Singer, 1986).

In this classification for this study, lode deposits are grouped into the hierarchic levels of metallogenic taxons according to such their stable features as: (a) environment of formation of host and geneticallyrelated rocks, (b) genetic features of the deposit, and (c) mineral and (or) elemental composition of the ore. The six hierarchial levels are as follows.

Group of deposits

Class of deposits

Clan of deposits

Deposit types (models)

The deposit models are subdivided into the following four large groups according to major geological rock-forming processes (Table 1): (1) deposits related to magmatic processes; (2) deposits related to hydrothermal-sedimentary processes; (3) deposits related to metamorphic processes; (4) deposits related to surficial processes and (6) exotic deposits. Each group includes several classes. For example, the group of deposits related to magmatic processes includes two classes: (1) those related to intrusive rocks; and (2) those related to extrusive rocks. Each class includes several clans, and so on. The most detailed subdivisions are for magmatic-related deposits because they are the most abundant in the project area. In the below classification, lode deposit types models that share a similar origin, such as magnesian and (or) calcic skarns, or porphyry deposits, are grouped together under a single genus with several types (or species) within the genus.

Some of the below deposit models differ from cited descriptions. For example, the Bayan Obo type was described previously as a carbonatite-related deposit. However, modern isotopic, mineralogical, and geological data recently obtained by Chinese geologists have resulted in a new interpretation of the deposit origin. These new data indicate that the deposit consists of ores that formed during Mesoproterozoic sedimentary-exhalative process, and along with coeval metasomatic activity, sedimentary diagenesis of dolomite, and alteration. The sedimentary-exhalative process consisted of both sedimentation and metasomatism. Later deformation, especially during the Caledonian orogeny, further enriched the ore. Consequently, the Bayan Obo deposit type is herein described as related to sedimentary-exhalative processes, not to magmatic processes. However, magmatic processes also played an important role in deposit formation. Consequently, this deposit model is part of the family of polygenetic carbonate-hosted deposits. Similar revisions are made for carbonatehosted $\mathrm{Hg}-\mathrm{Sb}$ and other deposit models.

Table 1. Hierarchial ranking of mineral deposit models.

Deposits related to magmatic processes

Deposits related to intrusive magmatic rocks

I. Deposits related to mafic and ultramafic intrusions

A. Deposits associated with differentiated mafic-ultramafic complexes

Mafic-ultramafic related $\mathrm{Cu}-\mathrm{Ni}-\mathrm{PGE}$

Mafic-ultramafic related $\mathrm{Ti}-\mathrm{Fe}( \pm \mathrm{V})$

Zoned mafic-ultramafic Cr-PGE

B. Deposits associated with ophiolitic complexes 
Podiform chromite

Serpentinite-hosted asbestos

C. Deposits associated with anorthosite complexes

Anorthosite apatite-Ti-Fe-P

D. Deposits associated with kimberlite

Diamond-bearing kimberlite

II. Deposits related to intermediate and felsic intrusions

A. Pegmatite

Muscovite pegmatite

REE-Li pegmatite

B. Greisen and quartz vein

Fluorite greisen

Sn-W greisen, stockwork, and quartz vein

W-Mo-Be greisen, stockwork, and quartz vein

C. Alkaline metasomatite

Ta-Nb-REE alkaline metasomatite

D. Skarn (contact metasomatic)

Au skarn

Boron (datolite) skarn

Carbonate-hosted asbestos

Co skarn

$\mathrm{Cu}( \pm \mathrm{Fe}, \mathrm{Au}, \mathrm{Ag}, \mathrm{Mo})$ skarn

Fe skarn

Fe-Zn skarn

Sn skarn

Sn-B (Fe) skarn (ludwigite)

$\mathrm{W} \pm \mathrm{Mo} \pm \mathrm{Be}$ skarn

$\mathrm{Zn}-\mathrm{Pb}( \pm \mathrm{Ag}, \mathrm{Cu})$ skarn

E. Porphyry and granitoid pluton-hosted deposit

Cassiterite-sulfide-silicate vein and stockwork

Felsic plutonic U-REE

Granitoid-related $\mathrm{Au}$ vein

Polymetallic $\mathrm{Pb}-\mathrm{Zn} \pm \mathrm{Cu}( \pm \mathrm{Ag}, \mathrm{Au})$ vein and stockwork

Porphyry $\mathrm{Au}$

Porphyry $\mathrm{Cu}( \pm \mathrm{Au})$

Porphyry $\mathrm{Cu}-\mathrm{Mo}( \pm \mathrm{Au}, \mathrm{Ag})$

Porphyry Mo $( \pm \mathrm{W}, \mathrm{Bi})$

Porphyry Sn

III. Deposits related to alkaline intrusions

A. Carbonatite-related deposits

Apatite carbonatite

Fe-REE carbonatite

$\mathrm{Fe}-\mathrm{Ti}( \pm \mathrm{Ta}, \mathrm{Nb}, \mathrm{Fe}, \mathrm{Cu}$, apatite) carbonatite

Phlogopite carbonatite

$\mathrm{REE}( \pm \mathrm{Ta}, \mathrm{Nb}, \mathrm{Fe})$ carbonatite

B. Alkaline-silisic intrusions related deposits

Alkaline complex-hosted Au

Peralkaline granitoid-related $\mathrm{Nb}-\mathrm{Zr}$-REE

Albite syenite-related REE

Ta-Li ongonite

C. Alkaline-gabbroic intrusion-related deposits

Charoite metasomatite

Magmatic and metasomatic apatite

Magmatic graphite

Magmatic nepheline

Deposits related to extrusive rocks

IV. Deposits related to marine extrusive rocks 
A. Massive sulfide deposits

Besshi $\mathrm{Cu}-\mathrm{Zn}-\mathrm{Ag}$ massive sulfide

Cyprus $\mathrm{Cu}-\mathrm{Zn}$ massive sulfide

Korean $\mathrm{Pb}-\mathrm{Zn}$ massive sulfide

Volcanogenic $\mathrm{Cu}-\mathrm{Zn}$ massive sulfide (Urals type)

Volcanogenic $\mathrm{Zn}-\mathrm{Pb}-\mathrm{Cu}$ massive sulfide (Kuroko, Altai types)

B. Volcanogenic-sedimentary deposits

Volcanogenic-hydrothermal-sedimentary massive sulfide $\mathrm{Pb}-\mathrm{Zn}( \pm \mathrm{Cu})$

Volcanogenic-sedimentary Fe

Volcanogenic-sedimentary Mn

V. Deposits related to subaerial extrusive rocks

A. Deposits associated with mafic extrusive rocks and dike complexes

$\mathrm{Ag}-\mathrm{Sb}$ vein

Basaltic native $\mathrm{Cu}$ (Lake Superior type)

$\mathrm{Hg}-\mathrm{Sb}-\mathrm{W}$ vein and stockwork

Hydrothermal Iceland spar

$\mathrm{Ni}-\mathrm{Co}$ arsenide vein

Silica-carbonate (listvenite) $\mathrm{Hg}$

Trap related Fe skarn (Angara-Ilim type)

B. Deposits associated with felsic to intermediate extrusive rocks

Au-Ag epithermal vein

$\mathrm{Ag}-\mathrm{Pb}$ epithermal vein

Au potassium metasomatite (Kuranakh type)

Barite vein

Be tuff

Carbonate-hosted As-Au metasomatite

Carbonate-hosted fluorspar

Carbonate-hosted $\mathrm{Hg}-\mathrm{Sb}$

Clastic sediment-hosted $\mathrm{Hg} \pm \mathrm{Sb}$

Epithermal quartz-alunite

Fluorspar vein

Hydrothermal-sedimentary fluorite

Limonite from spring water

Mn vein

Polymetallic $(\mathrm{Pb}, \mathrm{Zn} \pm \mathrm{Cu}, \mathrm{Ba}, \mathrm{Ag}, \mathrm{Au})$ volcanic-hosted metasomatite

Polymetallic $(\mathrm{Pb}, \mathrm{Zn}, \mathrm{Ag})$ carbonate-hosted metasomatite

Rhyolite-hosted Sn

Sulfur-sulfide (S, $\left.\mathrm{FeS}_{2}\right)$

Volcanic-hosted Au-base-metal metasomatite

Volcanic-hosted $\mathrm{Hg}$

Volcanic-hosted U

Volcanic-hosted zeolite

Deposits related to hydrothermal-sedimentary sedimentary processes

VI. Stratiform and stratabound deposits

Bedded barite

Carbonate-hosted $\mathrm{Pb}-\mathrm{Zn}$ (Mississippi valley type)

Sediment-hosted $\mathrm{Cu}$

Sedimentary exhalative Pb-Zn (SEDEX)

VII. Sedimentary rock-hosted deposits

Chemical-sedimentary Fe-Mn

Evaporate halite

Evaporate sedimentary gypsum

Sedimentary bauxite

Sedimentary celestite

Sedimentary phosphate

Sedimentary Fe-V

Sedimentary siderite $\mathrm{Fe}$ 
Stratiform Zr (Algama Type)

VIII. Polygenic carbonate-hosted deposits

Polygenic REE-Fe-Nb deposits (Bayan-Obo type)

Deposits related to metamorphic processes

IX. Sedimentary-metamorphic deposits

Banded iron formation (BIF, Algoma Fe)

Banded iron formation (BIF, Superior Fe)

Homestake Au

Sedimentary-metamorphic borate

Sedimentary-metamorphic magnesite

$\mathrm{X}$. Deposits related to regionally metamorphosed rocks

$\mathrm{Au}$ in black shale

$\mathrm{Au}$ in shear zone and quartz vein

Clastic-sediment-hosted Sb-Au

$\mathrm{Cu}-\mathrm{Ag}$ vein

Piezoquartz

Rhodusite asbestos

Talc (magnesite) replacement

Metamorphic graphite

Metamorphic sillimanite

Phlogopite skarn

Deposits related to surficial proceses

XI. Residual deposts

Bauxite (karst type)

Laterite $\mathrm{Ni}$

Weathering crust $\mathrm{Mn}( \pm \mathrm{Fe})$

Weathering crust and karst phosphate

Weathering crust carbonatite REE-Zr-Nb-Li

XII. Depositional deposits

Placer and paleoplacer $\mathrm{Au}$

Placer diamond

Placer PGE

Placer Sn

Placer Ti-Zr

REE and Fe oolite

Exotic deposits

Impact diamond

\section{Acknowledgements}

For the preparation of this report, we thank the many geologists who have worked with us for their valuable expertise in each region of Northeast Asia. We also thank managers N.L. Dobretsov, L.C. Gundersen, P.P. Hearn, K. Johnson, R. Koski, L.P. Leahy, J. Medlin, M. Power, and J.N. Weaver for their encouragement and support of the project. We thank Russian interpreters Tatiana Bounaeva and Elena Koltunova for their skill and assistance during long and complex scientific dialogues, and for translation of complex geologic descriptions and references.

\section{References Cited}

Ariunbileg, Sodov, Biryul'kin' G.V., Byamba' Jamba, Davydov, Y.V., Dejidmaa, Gunchin, Distanov, E.G.,
Dorjgotov, Gamyanin, G.N., Gerel, Ochir, Fridovskiy, V.Yu., Gotovsuren' Ayurzana, Hwang, Duk Hwan, Kochnev, A.P., Kostin, A.V., Kuzmin, M.I., Letunov, S.A., Li, Jiliang, Li, Xujun, Malceva, G.D., Melnikov, V.D., Nikitin, V.M., Obolenskiy, A.A., Ogasawara, Masatsugu, Orolmaa, Demberel, Parfenov, L.M.,. Popov, N.V., Prokopiev, A.V., Ratkin, V.V., Rodionov, S.M., Seminskiy, Z.V., Shpikerman, V.I., Smelov, A.P., Sotnikov, V.I., Spiridonov, A.V., Stogniy, V.V., Sudo, Sadahisa, Sun, Fengyue, Sun, Jiapeng, Sun, Weizhi,. Supletsov, V.M., Timofeev, V.F., Tyan, O.A., Vetluzhskikh, V.G., Xi, Aihua, Yakovlev, Y.V., Yan, Hongquan, Zhizhin, V.I., Zinchuk, N.N., and Zorina, L.M., 2003, Databases on significant metalliferous and selected non-metalliferous lode deposits, and selected placer districts for Northeast Asia, in Nokleberg, W.J., and 10 others, eds., Preliminary Publications Book 2 from Project on Mineral Resources, Metallogenesis, and Tectonics of 
Northeast Asia: U.S. Geological Survey Open-File Report 03-203 (CD-ROM).

Bilibin, Yu.A., 1955, Metallogenic provinces and metallogenic epochs: Moscow, Gosgeoltechizdat, 356 p. (in Russian).

Coney, P.J., Jones, D.L., and Monger, J.W.H., 1980, Cordilleran suspect terranes: Nature, v. 288, p. 329333.

Cox, D.P., 1993, Estimation of undiscovered deposits in quantitative mineral resource assessmentsexamples from Venezuela and Puerto Rico: Nonrenewable Resources, v. 2, no. 2, p. 82-91.

Cox, D.P. and Singer, D.A., eds., 1986, Mineral deposit models: U.S. Geological Survey Bulletin 1693, 379 p.

Dobretsov, N.L., and Kirdyashkin, A.G., 1994, Deep level geodynamics. Siberian Branch, Russian Academy of Sciences Press, Novosibirsk, 299 p. (in Russian).

Guild, P.W., 1978, Metallogenic maps; principles and progress: Global Tectonics Metallogeny, v. 1, no. 10 , p. $10-15$.

Howell, D.G., Jones, D.L., and Schermer, E.R., 1985, Tectonostratigraphic terranes of the Circum-Pacific region: Principles of terrane analysis, in Howell, D.G., ed., Tectonostratigraphic terranes of the Circum-Pacific region: Circum-Pacific Council for Energy and Mineral Resources, Houston, Texas, p. 3-31.

Itsikson, M.I., 1973, Metallogeny of planetary volcanogenic belts of Circum-Pacific: Evolution of volcanism in Earth's history: Nauka, Moscow, p.230-232 (in Russian).

Itsikson, M.I., 1979, Metallogenic zoning of CircumPacific: Nauka, Moscow, 232 p. (in Russian).

Itsikson, M.I., Krasny, L.I., and Matveenko, V.T., 1965, Volcanic belts of Circum-Pacific and their Metallogeny, in Ore-bearing Capacity of Volcanogenic Formations: Nedra, Moscow, p.181196 (in Russian).

Jones, D.L., Howell, D.G., Coney, P.J., and Monger, J.W.H., 1983, Recognition, character, and analysis of tectonostratigraphic terranes in western North America, in Hashimoto, M., and Uyeda, S., eds., Accretion tectonics in the circum-Pacific regions; Proceedings of the Oji International Seminar on Accretion Tectonics, Japan, 1981: Advances in Earth and Planetary Sciences, Tokyo, Terra Scientific Publishing Company, p. 21-35.

Koroteev, V.A., ed., 1996, Metallogeny of fold system with respect to plate tectonics: Urals Branch, Russian Academy of Sciences Press, Ekaterinburg, 248 p. (in Russian).

Ludington, S., and Cox, D., 1996, Data base for a national mineral-resource assessment of undiscovered deposits of gold, silver, copper, lead, and zinc in the conterminous United States by U.S. Geological Survey Minerals Team: U.S. Geological Survey Open-File Report 96-96, CD-ROM.

Mitchell, A.G., and Garson, M.S., 1981, Mineral deposits and global tectonic settings: Academic Press, London, $421 \mathrm{p}$.

Monger, J.W.H., and Berg, H.C., 1987, Lithotectonic terrane map of western Canada and southeastern Alaska: U. S. Geological Survey Miscellaneous
Field Studies Map MF-1874-B, 1 sheet, scale $1: 2,500,000,12 \mathrm{p}$.

Nokleberg, W.J., Bundtzen, T.K., Berg, H.C., Brew, D.A., Grybeck, Donald, Robinson, M.S., Smith, T.E., Yeend, Warren, 1994a, Metallogeny and major mineral deposits of Alaska, in Plafker, G. and Berg, H.C., eds., The Geology of Alaska: Boulder, Colorado, Geological Society of America: The Geology of North America, v. G1, p. 855-904.

Nokleberg, W.J., Parfenov, L.M., Monger, J.W.H., Baranov, B.V., Byalobzhesky, S.G. Bundtzen, T.K., Feeney, T.D., Fujita, Kazuya, Gordey, S.P., Grantz, A., Khanchuk, A.I., Natal'in, B.A. Natapov, L.M., Norton, 1.0., Patton, W.W. Jr., Planer, G., Csholl, D.W., Sokolov, S.D., Sosunov, G.M., Stone, D.B., Tabor, R.W., Tsukanov, N.V., Vallier, T.L. and Wakita, Koji, 1994b, Circum-North Pacific tectonostratigraphic terrane map: U.S. Geological Survey Open-File Report 94-714, 221 pages, 2 sheets, scale $1: 5,000,000 ; 2$ sheets, scale 1 : $10,000,000$.

Nokleberg, W.J., Parfenov, L.M., Monger, J.W.H., Norton, I.0. Khanchuk, A.I., Stone, D.B., Scotese, C.R., Scholl. D.W., and Fujita, K., 2001, Phanerozoic tectonic evolution of the Circum-North Pacific: U.S. Geological Survey Professional Paper 1626, $122 \mathrm{p}$

Nokleberg, W.J., Bundtzen, T.K., Grybeck, D., Koch, R.D., Eremin, R.A., Rozenblum, I.S., Sidorov, A.A., Byalobzhesky, S.G., Sosunov, G.M., Shpikennan, V.I., and Gorodinsky, M.E., 1993, Metallogenesis of mainland Alaska and the Russian Northeast: Mineral deposit maps, models, and tables, metallogenic belt maps and interpretation, and references cited: U.S. Geological Survey Open-File Report 93-339, 222 pages, 1 map, scale 1:4, 000,000, 5 maps, scale 1:10,000,000.

Nokleberg, W.J., West, T.D., Dawson, K.M., Shpikerman, V.I., Bundtzen, T.K., Parfenov, L.M., Monger, J.W.H., Ratkin, V.V., Baranov, B.V., Byalobzhesky, S.G., Diggles, M.F., Eremin, R.A., Fujita, K., Gordey, S.P., Gorodinskiy, M.E., Goryachev, N.A., Feeney, T.D., Frolov, Y.F., Grantz, A., Khanchuk, A.I., Koch, R.D., Natalin, B.A., Natapov, L.M., Norton, I.O., Patton, W.W. Jr., Plafker, G., Pozdeev, A.I., Rozenblum, I.S., Scholl, D.W., Sokolov, S.D., Sosunov, G.M., Stone, D.V., Tabor, R.W., Tsukanov, N.V., and Vallier, T.L., 1998, Summary terrane, mineral deposit, and metallogenic belt maps of the Russian Far East, Alaska, and the Canadian Cordillera: U.S. Geological Survey Open-File Report 98-136, CDROM.

Obolenskiy, A.A., Rodionov, S.M., Ariunbileg, Sodov, Dejidmaa, Gunchin, Distanov, E.G., Dorjgotov, Dangindorjiin, Gerel, Ochir, Hwang, Duk Hwan, Sun, Fengyue, Gotovsuren, Ayurzana, Letunov, S.N., Li, Xujun, Nokleberg, W.J., Ogasawara, Masatsugu, Seminsky, Z.V., Smelov, A.P., Sotnikov, V.I., Spiridonov, A.A., Zorina, L.V., and Yan, Hongquan, 2003, Mineral deposit models for Northeast Asia, in Nokleberg, W.J., and 10 others, eds.,: Preliminary Publications Book 2 from Project on Mineral Resources, Metallogenesis, and 
Tectonics of Northeast Asia: U.S. Geological Survey Open-File Report 03-203 (CD-ROM), 47 p.

Obolenskiy, A.A., Rodionov, S.M., Parfenov, L.M., Kuzmin, M.I., Distanov, E.G., Sotnikov, V.I., Seminskiy, Zh.V., Spiridonov, A.M., Stepanov, V.A., Khanchuk, A.I., Nokleberg, W.J., Tomurtogoo, O., Dejidmaa, G., Hongquan, Y., Fengyue, S., Hwang, D.H., and Ogasawara, M., 2001, Metallogenic belt map of Northeast Asia [abs.]: Joint $6^{\text {th }}$ Biennial SGA-SEG Meeting Program with abstracts, in Piestrzynski, Adam., and others, eds., Mineral Deposits at the Beginning of the 21st Century: Proceedings of Joint Sixth Biennial SGA-SEG Meeting, Krakow, Poland, A.A. Balkema Publishers, p.1133-1135.

Obruchev, V.V., 1928, Various investigations on ore deposit systematics: Journal of Mineralogy, Geology, and Paleontology, v. A., no. 4, p. 143-146 (in German).

Parfenov, L.M., Vetluzhskikh, V.G., Gamyanin, G.N., Davydov, Yu.V., Deikunenko, A.V., Kostin, A.V., Nikitin, V.M., Prokopyev, A.V., Smelov, A.P., Supletsov, V.M., Timofeev, V.F., Fridovsky, V.YU., Kholmogorov, A.I., Yakovlev, Ya.V., 1999, Metallogenic zonation of the territory of Sakha Republic: Pacific Ocean Geology, no. 2, p. 8-40.

Parfenov, L.M., Vetluzhskikh, V.G., Gamyanin, G.N., Davydov, Yu.V., Deikunenko, A.V., Kostin, A.V., Nikitin, V.M., Prokopyev, A.V., Smelov, A.P., Supletsov, V.M., Timofeev, V.F., Fridovsky, V.YU., Kholmogorov, A.I., Yakovlev, Ya.V., 1999, Metallogenic zonation of the territory of Sakha Republic: Pacific Ocean Geology, no. 2, p. 8-40.

Plyuschev, E.V., ed., 2001, Ore knots of Russia: VSEGEI, Saint-Petersburg, 416 p. (in Russian).

Pratt, W.P., ed., 1981, Metallic mineral-resource potential of the Rolla quadrangle, Missouri, as appraised in September 1980: U.S. Geological Survey Open-File Report 81-518, 77 p., 11 plates, scale 1:250,000.

Radkevich, E.A., 1982, Metallogeny of Circum-Pacific ore belt, in Metallogeny of Circum-Pacific: Far Eastern Branch, U.S.S.R. Academy of Sciences, p.3-16 (in Russian).

Remane, Jurgen, 1998, Explanatory note to global stratigraphic chart, in Circular of International Subcommission on Stratigraphic Classification (ISSC) of IUGS Commission on Stratigraphy, Appendix B: International Union of Geological Sciences (IUGS) Commission on Stratigraphy, v. 93, $11 \mathrm{p}$.

Rodionov, S.M., and Nokleberg, W.J., 2000, Mineral deposit models for Northeast Asia [abs.], in Mineral Resources and Tectonics of Northeast Asia: ITIT International Symposium June 8-9, Abstracts, AIST Research Center, Tsukuba, Japan, p. 51-53.

Rodionov, S.M., Obolenskiy, A.A., Khanchuk, A.I., Dejidmaa, G., Hongquan, Y., Hwang, D.H., and Nokleberg, W.J., 2000, Metallogenic belts of Northeast Asia: Definitions, principles, and examples [abs.], in Mineral Resources and Tectonics of Northeast Asia: ITIT International Symposium, June 8-9, Abstracts. AIST Research Center, Tsukuba, Japan, p. 82-83.
Scheglov, A.D., 1980, Basis of metallogenic analyses: Nedra, Moscow, 431p (in Russian).

Shatalov, E.G., 1965, Principles of metallogenic map compilation, in Questions of Metallogeny: Nedra, Moscow, p.45-61 (in Russian).

Singer, D.A., 1993, Development of grade and tonnage models for different deposit types, in Kirkham, R.V., Sinclair, R.V., Thorpe, W.D., and Duke, J.M., eds., Mineral deposit modeling: Geological Association Canada Special Paper 40, 27 p. 21-30.

Singer, D.A., 1994, The relationship of estimated number of undiscovered deposits to grade and tonnage models in three-part mineral resource assessments: 1994 Intern. Assoc Math. Geology Annual Conference, Papers and Entended Abstracts, Oct. 3-5, 1994, Mount Tremblant, Quebec, Canada, p. 325-326.

Scotese, C.R., Nokleberg, W.J., Monger, J.W.H., Norton, I.O., Parfenov, L.M., Bundtzen, T.K., Dawson, K.M., Eremin, R.A., Frolov, Y.F., Fujita, Kazuya, Goryachev, N.A., Khanchuk, A.I., Pozdeev, A.I., Ratkin, V.V., Rodinov, S.M., Rozenblum, I.S., Shpikerman, V.I., Sidorov, A.A., and Stone, D.B., 2001, in Nokleberg, W..J. and Diggles, M.F., eds., Dynamic Computer Model for the Metallogenesis and Tectonics of the Circum-North Pacific: U.S. Geological Survey Open-File Report 01-161, CDROM.

Smirnov, V.I., 1969, Geology of useful minerals: Nedra, Moscow, 687 p. (in Russian).

Sukhov, V.I., Bakulin, Yu.I., Loshak, N.P., Khitrunov, A.T., Rodionova, L.N., and Karas, N.A., 2000, Metallogeny of Russian Far East: DVIMS Publishing House, Khabarovsk, 217p (in Russian).

Tomson, I.N., 1988, Metallogeny of ore regions. Nedra, Moscow, $215 \mathrm{p}$ (in Russian).

Wheeler, J.O., Brookfield, A.J., Gabrielse, H., Monger, J.W.H., Tipper, H.W., and Woodsworth, G.J., 1988, Terrane map of the Canadian Cordillera: Geological Survey of Canada Open File Report 1894, scale $1: 2,000,000,9 \mathrm{p}$.

Zonenshain, L.P., Kuzmin, M.I. and Natapov, L.M. 1992, Plate tectonics and ore deposits in Northern Eurasia (the former USSR) [abs.]: Colorado School of Mines Quarterly Review, v. 92, no. 2, p. 13. 
Table 2. Summary table for significant metalliferous and selected nonmetalliferous lode deposits of Northeast Asia. Deposits listed in numerical order by map row and map column for quadrants of latitude and longitude.

\begin{tabular}{|c|c|c|c|c|c|c|}
\hline Row & Col. & No. & Deposit Name & Mineral Deposit Type & Major Metals & Metallogenic Belt \\
\hline $\mathrm{H}$ & 52 & 1 & Shika & $\mathrm{Ag}-\mathrm{Sb}$ vein & $\mathrm{Sb}$ & Outer Zone Southwest Japan \\
\hline $\mathrm{H}$ & 52 & 2 & Yamagano & Au-Ag epithermal vein & $\mathrm{Au}$ & Kyushu \\
\hline $\mathrm{H}$ & 52 & 3 & Kushikino & Au-Ag epithermal vein & $\mathrm{Au}, \mathrm{Ag}$ & Kyushu \\
\hline $\mathrm{H}$ & 52 & 4 & Suzuyama & Sn-W greisen, stockwork, and quartz vein & Sn & Outer Zone Southwest Japan \\
\hline $\mathrm{H}$ & 52 & 5 & Akeshi & Au-Ag epithermal vein & $\mathrm{Au}, \mathrm{Ag}$ & Kyushu \\
\hline $\mathrm{H}$ & 52 & 6 & Iwato & $\mathrm{Au}-\mathrm{Ag}$ epithermal vein & $\mathrm{Au}, \mathrm{Ag}$ & Kyushu \\
\hline $\mathrm{H}$ & 52 & 7 & Kasuga & $\mathrm{Au}-\mathrm{Ag}$ epithermal vein & $\mathrm{Au}, \mathrm{Ag}$ & Kyushu \\
\hline $\mathrm{H}$ & 52 & 8 & Nitta-Yakushima & W-Mo-Be greisen, stockwork, and quartz vein & $\mathrm{W}$ & Outer Zone Southwest Japan \\
\hline I & 52 & 1 & Kyeongju & Polymetallic $\mathrm{Pb}-\mathrm{Zn} \pm \mathrm{Cu}( \pm \mathrm{Ag}, \mathrm{Au})$ vein and stockwork & Mo & Gyeongpuk \\
\hline I & 52 & 10 & Mulkum & $\begin{array}{l}\text { Polymetallic }(\mathrm{Pb}, \mathrm{Zn} \pm \mathrm{Cu}, \mathrm{Ba}, \mathrm{Ag}, \mathrm{Au}) \text { volcanic-hosted } \\
\text { metasomatite }\end{array}$ & $\mathrm{Fe}$ & Gyeongnam \\
\hline I & 52 & 11 & Dongbogwang & W-Mo-Be greisen, stockwork, and quartz vein & $\mathrm{W}$ & Gyeongnam \\
\hline I & 52 & 12 & Yongho & $\mathrm{Cu}-\mathrm{Ag}$ vein & $\mathrm{Cu}$ & Gyeongnam \\
\hline $\mathrm{I}$ & 52 & 13 & Akimoto & Volcanogenic-sedimentary Mn & $\mathrm{Mn}$ & Sambagawa-Chichibu-Shimanto \\
\hline I & 52 & 13 & Dongjin & $\mathrm{Au}$ in shear zone and quartz vein & $\mathrm{Au}, \mathrm{Ag}$ & Sannae \\
\hline I & 52 & 14 & Masan & $\begin{array}{l}\text { Polymetallic }(\mathrm{Pb}, \mathrm{Zn} \pm \mathrm{Cu}, \mathrm{Ba}, \mathrm{Ag}, \mathrm{Au}) \text { volcanic-hosted } \\
\text { metasomatite }\end{array}$ & $\mathrm{Cu}, \mathrm{Pb}, \mathrm{Zn}$ & Gyeongnam \\
\hline I & 52 & 15 & Kuryong & $\begin{array}{l}\text { Polymetallic }(\mathrm{Pb}, \mathrm{Zn} \pm \mathrm{Cu}, \mathrm{Ba}, \mathrm{Ag}, \mathrm{Au}) \text { volcanic-hosted } \\
\text { metasomatite }\end{array}$ & $\mathrm{Fe}, \mathrm{Cu}$ & Gyeongnam \\
\hline I & 52 & 16 & Jinju & Polymetallic $\mathrm{Pb}-\mathrm{Zn} \pm \mathrm{Cu}( \pm \mathrm{Ag}, \mathrm{Au})$ vein and stockwork & $\mathrm{Pb}, \mathrm{Zn}$ & Gyeongnam \\
\hline I & 52 & 17 & Haman-Gunpuk & $\begin{array}{l}\text { Polymetallic }(\mathrm{Pb}, \mathrm{Zn} \pm \mathrm{Cu}, \mathrm{Ba}, \mathrm{Ag}, \mathrm{Au}) \text { volcanic-hosted } \\
\text { metasomatite }\end{array}$ & $\mathrm{Cu}, \mathrm{Pb}, \mathrm{Zn}$ & Gyeongnam \\
\hline I & 52 & 18 & Sannae & $\mathrm{Ni}-\mathrm{Co}$ arsenide vein & $\mathrm{Ni}, \mathrm{Co}$ & Sannae \\
\hline I & 52 & 19 & Yungchang 1 & Polymetallic $\mathrm{Pb}-\mathrm{Zn} \pm \mathrm{Cu}( \pm \mathrm{Ag}, \mathrm{Au})$ vein and stockwork & $\mathrm{Cu}, \mathrm{Pb}, \mathrm{Zn}$ & Gyeongnam \\
\hline $\mathrm{I}$ & 52 & 2 & Ulsan & Fe skarn & $\mathrm{Fe}$ & Gyeongnam \\
\hline $\mathrm{I}$ & 52 & 20 & Goseong & $\mathrm{Cu}-\mathrm{Ag}$ vein & $\mathrm{Cu}, \mathrm{Au}, \mathrm{Ag}$ & Gyeongnam \\
\hline I & 52 & 21 & Koksung & Metamorphic graphite & Graphite & Koksung \\
\hline I & 52 & 22 & Tongyoung & $\mathrm{Au}$ in shear zone and quartz vein & $\mathrm{Au}, \mathrm{Ag}$ & Gyeongnam \\
\hline $\mathrm{I}$ & 52 & 23 & Samdong & Porphyry Mo $( \pm \mathrm{W}, \mathrm{Sn}, \mathrm{Bi})$ & $\mathrm{Mo}, \mathrm{Cu}$ & Gyeongnam \\
\hline I & 52 & 24 & Taishu & Polymetallic $\mathrm{Pb}-\mathrm{Zn} \pm \mathrm{Cu}( \pm \mathrm{Ag}, \mathrm{Au})$ vein and stockwork & $\mathrm{Zn}, \mathrm{Pb}$ & Hokuriku-Sanin \\
\hline I & 52 & 25 & Hoshino & Au-Ag epithermal vein & $\mathrm{Au}$ & Kyushu \\
\hline I & 52 & 26 & Taio & $\mathrm{Au}-\mathrm{Ag}$ epithermal vein & $\mathrm{Au}, \mathrm{Ag}$ & Kyushu \\
\hline $\mathrm{I}$ & 52 & 27 & Hoei & Sn skarn & Sn & Outer Zone Southwest Japan \\
\hline I & 52 & 28 & Shinkiura & Sn skarn & Sn & Outer Zone Southwest Japan \\
\hline $\mathrm{I}$ & 52 & 29 & Obira & Cassiterite-sulfide-silicate vein and stockwork & Sn, As & Outer Zone Southwest Japan \\
\hline $\mathrm{I}$ & 52 & 3 & Darak & Polymetallic $\mathrm{Pb}-\mathrm{Zn} \pm \mathrm{Cu}( \pm \mathrm{Ag}, \mathrm{Au})$ vein and stockwork & $\mathrm{Pb}, \mathrm{Zn}$ & Gyeongpuk \\
\hline I & 52 & 30 & Mitate & Sn skarn & Sn & Outer Zone Southwest Japan \\
\hline $\mathrm{I}$ & 52 & 31 & Toroku & Sn skarn & Sn, As & Outer Zone Southwest Japan \\
\hline I & 52 & 32 & Makimine & Besshi $\mathrm{Cu}-\mathrm{Zn}-\mathrm{Ag}$ massive sulfide & $\mathrm{Cu}$ & Sambagawa-Chichibu-Shimanto \\
\hline I & 52 & 34 & Miyazaki-Matsuo & Polymetallic $\mathrm{Pb}-\mathrm{Zn} \pm \mathrm{Cu}( \pm \mathrm{Ag}, \mathrm{Au})$ vein and stockwork & As & Outer Zone Southwest Japan \\
\hline $\mathrm{I}$ & 52 & 35 & Saeki district & Volcanogenic-sedimentary Mn & $\mathrm{Mn}$ & Sambagawa-Chichibu-Shimanto \\
\hline $\mathrm{I}$ & 52 & 36 & Itsuki district & Volcanogenic-sedimentary Mn & $\mathrm{Mn}$ & Sambagawa-Chichibu-Shimanto \\
\hline I & 52 & 37 & Fuke & Au-Ag epithermal vein & $\mathrm{Au}, \mathrm{Ag}$ & Kyushu \\
\hline $\mathrm{I}$ & 52 & 38 & Ohkuchi & $\mathrm{Au}-\mathrm{Ag}$ epithermal vein & $\mathrm{Au}, \mathrm{Ag}$ & Kyushu \\
\hline
\end{tabular}




\begin{tabular}{|c|c|c|c|c|c|c|}
\hline I & 52 & 39 & Hishikari & Au-Ag epithermal vein & $\mathrm{Au}, \mathrm{Ag}$ & Kyushu \\
\hline I & 52 & 4 & Wolak & W-Mo-Be greisen, stockwork, and quartz vein & $\mathrm{W}, \mathrm{Mo}$ & Wolak \\
\hline I & 52 & 5 & Sasagatani & $\mathrm{Cu}( \pm \mathrm{Fe}, \mathrm{Au}, \mathrm{Ag}, \mathrm{Mo})$ skarn & $\mathrm{Cu}, \mathrm{Zn}$ & Inner Zone Southwest Japan \\
\hline I & 52 & 6 & Donggok & Polymetallic $\mathrm{Pb}-\mathrm{Zn} \pm \mathrm{Cu}( \pm \mathrm{Ag}, \mathrm{Au})$ vein and stockwork & $\mathrm{Pb}, \mathrm{Zn}$ & Gyeongnam \\
\hline I & 52 & 7 & Yanggudong & $\mathrm{Cu}-\mathrm{Ag}$ vein & $\mathrm{Cu}, \mathrm{Pb}, \mathrm{Zn}$ & Gyeongnam \\
\hline I & 52 & 8 & Gwymyeong & $\begin{array}{l}\text { Polymetallic }(\mathrm{Pb}, \mathrm{Zn} \pm \mathrm{Cu}, \mathrm{Ba}, \mathrm{Ag}, \mathrm{Au}) \text { volcanic-hosted } \\
\text { metasomatite }\end{array}$ & $\mathrm{Pb}, \mathrm{Zn}$ & Gyeongnam \\
\hline I & 52 & 9 & Cheolma & $\mathrm{Au}$ in shear zone and quartz vein & $\mathrm{Au}, \mathrm{Ag}$ & Gyeongnam \\
\hline I & 53 & 1 & Hamayokokawa & Volcanogenic-sedimentary Mn & $\mathrm{Mn}$ & Mino-Tamba-Chugoku \\
\hline I & 53 & 10 & Yaei & Volcanogenic-sedimentary Mn & $\mathrm{Mn}$ & Mino-Tamba-Chugoku \\
\hline I & 53 & 11 & Tamba district & Volcanogenic-sedimentary Mn & $\mathrm{Mn}$ & Mino-Tamba-Chugoku \\
\hline I & 53 & 12 & Kaneuchi & W-Mo-Be greisen, stockwork, and quartz vein & $\mathrm{W}$ & Inner Zone Southwest Japan \\
\hline I & 53 & 13 & Tonoda district & Volcanogenic-sedimentary $\mathrm{Mn}$ & $\mathrm{Mn}$ & Mino-Tamba-Chugoku \\
\hline I & 53 & 14 & Iwami & $\mathrm{Au}-\mathrm{Ag}$ epithermal vein & $\mathrm{Cu}$ & Hokuriku-Sanin \\
\hline I & 53 & 15 & Nakase & $\mathrm{Ag}-\mathrm{Sb}$ vein & $\mathrm{Sb}, \mathrm{Au}, \mathrm{Ag}$ & Hokuriku-Sanin \\
\hline I & 53 & 16 & Otani & W-Mo-Be greisen, stockwork, and quartz vein & $\mathrm{W}, \mathrm{Cu}, \mathrm{Sn}$ & Inner Zone Southwest Japan \\
\hline I & 53 & 17 & Akenobe & Polymetallic $\mathrm{Pb}-\mathrm{Zn} \pm \mathrm{Cu}( \pm \mathrm{Ag}, \mathrm{Au})$ vein and stockwork & $\mathrm{Zn}, \mathrm{Cu}, \mathrm{Sn}$ & Inner Zone Southwest Japan \\
\hline I & 53 & 18 & Ikuno & Polymetallic $\mathrm{Pb}-\mathrm{Zn} \pm \mathrm{Cu}( \pm \mathrm{Ag}, \mathrm{Au})$ vein and stockwork & $\begin{array}{l}\mathrm{Ag}, \mathrm{Au}, \mathrm{Cu} \\
\mathrm{Zn}, \mathrm{Sn}, \mathrm{Pb}\end{array}$ & Inner Zone Southwest Japan \\
\hline I & 53 & 19 & Ningyotoge & Clastic-sediment-hosted U & $\mathrm{U}$ & Hokuriku-Sanin \\
\hline I & 53 & 2 & Nakatatsu & $\mathrm{Zn}-\mathrm{Pb}(\mathrm{Ag}, \mathrm{Cu}, \mathrm{W})$ skarn & $\mathrm{Zn}, \mathrm{Ag}, \mathrm{Pb}$ & Inner Zone Southwest Japan \\
\hline I & 53 & 20 & Yamatosuigin & Hg-Sb-W vein and stockwork & $\mathrm{Hg}$ & Outer Zone Southwest Japan \\
\hline I & 53 & 21 & Kamio & Volcanic-hosted Hg. & $\mathrm{Hg}$ & Outer Zone Southwest Japan \\
\hline I & 53 & 22 & Yanahara & Besshi $\mathrm{Cu}-\mathrm{Zn}-\mathrm{Ag}$ massive sulfide & Pyrite & Mino-Tamba-Chugoku \\
\hline I & 53 & 23 & Daito & W-Mo-Be greisen, stockwork, and quartz vein & Mo & Inner Zone Southwest Japan \\
\hline $\mathrm{I}$ & 53 & 24 & Seikyu & Porphyry Mo $( \pm \mathrm{W}, \mathrm{Sn}, \mathrm{Bi})$ & Mo & Inner Zone Southwest Japan \\
\hline $\mathrm{I}$ & 53 & 25 & Niu & $\mathrm{Hg}-\mathrm{Sb}-\mathrm{W}$ vein and stockwork & $\mathrm{Hg}$ & Outer Zone Southwest Japan \\
\hline I & 53 & 26 & Wakamatsu & Podiform chromite & $\mathrm{Cr}$ & Mino-Tamba-Chugoku \\
\hline $\mathrm{I}$ & 53 & 27 & Hirose & Podiform chromite & $\mathrm{Cr}$ & Mino-Tamba-Chugoku \\
\hline $\mathrm{I}$ & 53 & 28 & Komaki & W-Mo-Be greisen, stockwork, and quartz vein & Mo & Inner Zone Southwest Japan \\
\hline I & 53 & 29 & Iimori & Besshi $\mathrm{Cu}-\mathrm{Zn}-\mathrm{Ag}$ massive sulfide & $\mathrm{Cu}$ & Sambagawa-Chichibu-Shimanto \\
\hline I & 53 & 3 & Hiraiwa-Sasabora & Fluospar vein & Fluorite & Inner Zone Southwest Japan \\
\hline I & 53 & 30 & Yoshioka & Polymetallic $\mathrm{Pb}-\mathrm{Zn} \pm \mathrm{Cu}( \pm \mathrm{Ag}, \mathrm{Au})$ vein and stockwork & $\mathrm{Cu}, \mathrm{Ag}$ & Inner Zone Southwest Japan \\
\hline $\mathrm{I}$ & 53 & 31 & Omori & Au-Ag epithermal vein & $\mathrm{Ag}, \mathrm{Au}$ & Hokuriku-Sanin \\
\hline I & 53 & 32 & Obie & Polymetallic $\mathrm{Pb}-\mathrm{Zn} \pm \mathrm{Cu}( \pm \mathrm{Ag}, \mathrm{Au})$ vein and stockwork & $\mathrm{Cu}, \mathrm{Ag}$ & Inner Zone Southwest Japan \\
\hline $\mathrm{I}$ & 53 & 33 & Kishu & $\mathrm{Au}-\mathrm{Ag}$ epithermal vein & $\mathrm{Ag}, \mathrm{Au}, \mathrm{Cu}$ & Outer Zone Southwest Japan \\
\hline $\mathrm{I}$ & 53 & 34 & Myoho & Polymetallic $\mathrm{Pb}-\mathrm{Zn} \pm \mathrm{Cu}( \pm \mathrm{Ag}, \mathrm{Au})$ vein and stockwork & $\mathrm{Cu}$ & Outer Zone Southwest Japan \\
\hline I & 53 & 35 & Higashiyama & Besshi $\mathrm{Cu}-\mathrm{Zn}-\mathrm{Ag}$ massive sulfide & $\mathrm{Cu}$, Pyrite & Sambagawa-Chichibu-Shimanto \\
\hline $\mathrm{I}$ & 53 & 37 & Sazare & Besshi $\mathrm{Cu}-\mathrm{Zn}-\mathrm{Ag}$ massive sulfide & $\mathrm{Cu}$ & Sambagawa-Chichibu-Shimanto \\
\hline I & 53 & 38 & Shirataki & Besshi $\mathrm{Cu}-\mathrm{Zn}-\mathrm{Ag}$ massive sulfide & $\mathrm{Cu}$, Pyrite & Sambagawa-Chichibu-Shimanto \\
\hline I & 53 & 39 & Besshi & Besshi $\mathrm{Cu}-\mathrm{Zn}-\mathrm{Ag}$ massive sulfide & $\mathrm{Cu}, \mathrm{Au}, \mathrm{Ag}$ & Sambagawa-Chichibu-Shimanto \\
\hline $\mathrm{I}$ & 53 & 39 & Kootsu & Besshi $\mathrm{Cu}-\mathrm{Zn}-\mathrm{Ag}$ massive sulfide & $\mathrm{Cu}$, Pyrite & Sambagawa-Chichibu-Shimanto \\
\hline I & 53 & 4 & Higashimino district & Volcanogenic-sedimentary Mn & $\mathrm{Mn}$ & Mino-Tamba-Chugoku \\
\hline $\mathrm{I}$ & 53 & 40 & Ichinokawa & Clastic-sediment-hosted Sb-Au & $\mathrm{Sb}$ & Outer Zone Southwest Japan \\
\hline I & 53 & 41 & Kawayama & Besshi $\mathrm{Cu}-\mathrm{Zn}-\mathrm{Ag}$ massive sulfide & $\mathrm{Cu}, \mathrm{Zn}, \mathrm{Ag}, \mathrm{S}$ & Mino-Tamba-Chugoku \\
\hline I & 53 & 42 & Ananai district & Volcanogenic-sedimentary $\mathrm{Mn}$ & $\mathrm{Mn}$ & Sambagawa-Chichibu-Shimanto \\
\hline $\mathrm{I}$ & 53 & 43 & Kuga & $\mathrm{W} \pm \mathrm{Mo} \pm \mathrm{Be}$ skarn & $\mathrm{W}$ & Inner Zone Southwest Japan \\
\hline I & 53 & 44 & Fujigatani & $\mathrm{W} \pm \mathrm{Mo} \pm \mathrm{Be}$ skarn & W & Inner Zone Southwest Japan \\
\hline I & 53 & 45 & Iwakuni district & Volcanogenic-sedimentary $\mathrm{Mn}$ & $\mathrm{Mn}$ & Mino-Tamba-Chugoku \\
\hline I & 53 & 46 & Okuki & Cyprus $\mathrm{Cu}-\mathrm{Zn}$ massive sulfide & $\mathrm{Cu}$ & Sambagawa-Chichibu-Shimanto \\
\hline I & 53 & 47 & Uwajima district & Volcanogenic-sedimentary Mn & $\mathrm{Mn}$ & Sambagawa-Chichibu-Shimanto \\
\hline
\end{tabular}




\begin{tabular}{|c|c|c|c|c|c|c|}
\hline Row & Col. & No. & Deposit Name & Mineral Deposit Type & Major Metals & Metallogenic Belt \\
\hline I & 53 & 48 & Fujinokawa & $\mathrm{Ag}-\mathrm{Sb}$ vein & $\mathrm{Sb}$ & Outer Zone Southwest Japan \\
\hline I & 53 & 5 & Nishimino district & Volcanogenic-sedimentary Mn & $\mathrm{Mn}$ & Mino-Tamba-Chugoku \\
\hline $\mathrm{I}$ & 53 & 7 & Kune & Besshi Cu-Zn-Ag massive sulfide & $\mathrm{Cu}$ & Sambagawa-Chichibu-Shimanto \\
\hline $\mathrm{I}$ & 53 & 8 & Minenosawa & Besshi $\mathrm{Cu}-\mathrm{Zn}-\mathrm{Ag}$ massive sulfide & $\mathrm{Cu}, \mathrm{Zn}, \mathrm{Au}, \mathrm{Ag}$ & Sambagawa-Chichibu-Shimanto \\
\hline I & 53 & 9 & Kitatamba district & Volcanogenic-sedimentary $\mathrm{Mn}$ & $\mathrm{Mn}$ & Mino-Tamba-Chugoku \\
\hline I & 54 & 1 & Takara & $\begin{array}{l}\text { Volcanogenic } \mathrm{Zn}-\mathrm{Pb}-\mathrm{Cu} \text { massive sulfide (Kuroko, Altai } \\
\text { type) }\end{array}$ & $\mathrm{Zn}, \mathrm{Cu}$ & Northeast Japan \\
\hline I & 54 & 2 & Seikoshi & Au-Ag epithermal vein & $\mathrm{Ag}, \mathrm{Au}$ & Northeast Japan \\
\hline I & 54 & 3 & Mochikoshi & $\mathrm{Au}-\mathrm{Ag}$ epithermal vein & $\mathrm{Au}, \mathrm{Ag}$ & Northeast Japan \\
\hline $\mathrm{I}$ & 54 & 4 & Toi & Au-Ag epithermal vein & $\mathrm{Au}, \mathrm{Ag}$ & Northeast Japan \\
\hline $\mathrm{J}$ & 49 & 1 & $\begin{array}{l}\text { Yixingzai, Fanshi, Shanxi } \\
\text { Province }\end{array}$ & Volcanic-hosted Au-base-metal metasomatite & $\mathrm{Au}$ & Yanshan \\
\hline $\mathrm{J}$ & 49 & 10 & $\begin{array}{l}\text { Guopanliang, Baode } \\
\text { County, Shanxi Province }\end{array}$ & Sedimentary bauxite & $\mathrm{Al}$ & Shanxi \\
\hline $\mathrm{J}$ & 49 & 11 & Taiyuan, Shanxi Province & Evaporate sedimentary gypsum & Gypsum & Jinzhong \\
\hline $\mathrm{J}$ & 49 & 12 & $\begin{array}{l}\text { Yuanjiachun, Shanxi } \\
\text { Province }\end{array}$ & Banded iron formation (BIF, Superior Fe) & $\mathrm{Fe}$ & Luliangshan \\
\hline $\mathrm{J}$ & 49 & 13 & Sitou, Shaxi Province & Banded iron formation (BIF, Superior Fe) & $\mathrm{Fe}$ & Luliangshan \\
\hline $\mathrm{J}$ & 49 & 14 & Lingshi, Shanxi Province & Evaporate sedimentary gypsum & Gypsum & Jinzhong \\
\hline $\mathrm{J}$ & 49 & 15 & $\begin{array}{l}\text { Shigong, Xiaoyi, Shanxi } \\
\text { Province }\end{array}$ & Sedimentary bauxite & $\mathrm{Al}$ & Shanxi \\
\hline $\mathrm{J}$ & 49 & 16 & $\begin{array}{l}\text { Xiangwang, Xiaoyi, } \\
\text { Shanxi Province }\end{array}$ & Sedimentary bauxite & $\mathrm{Al}$ & Shanxi \\
\hline $\mathrm{J}$ & 49 & 17 & Re'er, Shanxi Province & Sedimentary bauxite & $\mathrm{Al}$ & Shanxi \\
\hline $\mathrm{J}$ & 49 & 18 & $\begin{array}{l}\text { Xihedi, Xiaoyi, Shanxi } \\
\text { Province }\end{array}$ & Sedimentary bauxite & $\mathrm{Al}$ & Shanxi \\
\hline $\mathrm{J}$ & 49 & 19 & $\begin{array}{l}\text { Duchun, Xiaoyi, Shanxi } \\
\text { Province }\end{array}$ & Sedimentary bauxite & $\mathrm{Al}$ & Shanxi \\
\hline $\mathrm{J}$ & 49 & 2 & $\begin{array}{l}\text { Baizhiyan, Shanxi } \\
\text { Province }\end{array}$ & Banded iron formation (BIF, Algoma Fe) & $\mathrm{Fe}$ & Wutai \\
\hline $\mathrm{J}$ & 49 & 20 & $\begin{array}{l}\text { Yangjiashan, Lishi } \\
\text { County, Shanxi Province }\end{array}$ & Sedimentary bauxite & $\mathrm{Al}$ & Shanxi \\
\hline $\mathrm{J}$ & 49 & 21 & $\begin{array}{l}\text { Xiangyi, Jiaokou County, } \\
\text { Shanxi Province }\end{array}$ & Sedimentary bauxite & $\mathrm{Al}$ & Shanxi \\
\hline $\mathrm{J}$ & 49 & 22 & Ke'er, Shanxi Province & Sedimentary bauxite & $\mathrm{Al}$ & Shanxi \\
\hline $\mathrm{J}$ & 49 & 23 & $\begin{array}{l}\text { Shanyangping, Daixian } \\
\text { County,Shanxi Province }\end{array}$ & Banded iron formation (BIF, Algoma Fe) & $\mathrm{Fe}$ & Wutai \\
\hline $\mathrm{J}$ & 49 & 3 & Jingangku, Shaxi Province & Banded iron formation (BIF, Algoma Fe) & $\mathrm{S}$ & Witai \\
\hline $\mathrm{J}$ & 49 & 4 & $\begin{array}{l}\text { Yangchaoping, Daixian } \\
\text { County, Shanxi Province }\end{array}$ & Banded iron formation (BIF, Algoma Fe) & $\mathrm{Fe}$ & Wutain \\
\hline $\mathrm{J}$ & 49 & 5 & $\begin{array}{l}\text { Tianqiao, Baode County, } \\
\text { Shanxi Province }\end{array}$ & Sedimentary bauxite & $\mathrm{Al}$ & Shanxi \\
\hline $\mathrm{J}$ & 49 & 6 & Hulishan, Shanxi Province & $\mathrm{Au}$ in shear zone and quartz vein & $\mathrm{Au}$ & Luliangshan \\
\hline $\mathrm{J}$ & 49 & 7 & $\begin{array}{l}\text { Baiquan, Yangquan, } \\
\text { Shanxi Province }\end{array}$ & Sedimentary bauxite & $\mathrm{Al}$ & Shanxi \\
\hline $\mathrm{J}$ & 49 & 8 & $\begin{array}{l}\text { Taihushi, Yangquan } \\
\text { County, Shanxi Province }\end{array}$ & Sedimentary bauxite & $\mathrm{Al}$ & Shanxi \\
\hline $\mathrm{J}$ & 49 & 9 & $\begin{array}{l}\text { Qianmuping, Yangquan } \\
\text { County, Shanxi Province }\end{array}$ & Sedimentary bauxite & $\mathrm{Al}$ & Shanxi \\
\hline $\mathrm{J}$ & 50 & 1 & Sijiaying, Hebei Province & Banded iron formation (BIF, Algoma Fe) & $\mathrm{Fe}$ & Jidong \\
\hline $\mathrm{J}$ & 50 & 10 & Xishimen, Wu'an, Hebei & Fe skarn & $\mathrm{Fe}$ & Hanxing \\
\hline
\end{tabular}




\begin{tabular}{|c|c|c|c|c|c|c|}
\hline & & & Province & & & \\
\hline $\mathrm{J}$ & 50 & 11 & $\begin{array}{l}\text { Zhongguan, Wu'an, Hebei } \\
\text { Province }\end{array}$ & Fe skarn & $\mathrm{Fe}$ & Hanxing \\
\hline $\mathrm{J}$ & 50 & 2 & $\begin{array}{l}\text { Dawan, Laiyuan County, } \\
\text { Hebei Province }\end{array}$ & Porphyry $\mathrm{Cu}-\mathrm{Mo}( \pm \mathrm{Au}, \mathrm{Ag})$ & Mo & Yanshan \\
\hline $\mathrm{J}$ & 50 & 3 & $\begin{array}{l}\text { Pingxingguan, Shanxi } \\
\text { Province }\end{array}$ & Banded iron formation (BIF, Algoma Fe) & $\mathrm{Fe}$ & Wutai \\
\hline $\mathrm{J}$ & 50 & 4 & $\begin{array}{l}\text { Tuling-Shihu, Lishou, } \\
\text { Hebei Province }\end{array}$ & Granitoid-related Au vein & $\mathrm{Au}$ & Yanshan \\
\hline $\mathrm{J}$ & 50 & 5 & $\begin{array}{l}\text { Jinling, Shandong } \\
\text { Province }\end{array}$ & Fe-Zn skarn & $\mathrm{Fe}$ & Laiwu \\
\hline $\mathrm{J}$ & 50 & 6 & $\begin{array}{l}\text { Zihe (Heiwang), } \\
\text { Shandong Province }\end{array}$ & Fe skarn & $\mathrm{Fe}$ & Laiwu \\
\hline $\mathrm{J}$ & 50 & 7 & Zibe, Shandong Province & Sedimentary bauxite & $\mathrm{Al}$ & Zibe 1 \\
\hline $\mathrm{J}$ & 50 & 8 & $\begin{array}{l}\text { Xishimen, Wuan, Hebei } \\
\text { Province }\end{array}$ & Fe skarn & $\mathrm{Fe}$ & Hanxing \\
\hline $\mathrm{J}$ & 50 & 9 & $\begin{array}{l}\text { Yushiwa, Wuan, Hebei } \\
\text { Province }\end{array}$ & Fe skarn & $\mathrm{Fe}$ & Hanxing \\
\hline $\mathrm{J}$ & 51 & 1 & $\begin{array}{l}\text { Huatong, Liaoning } \\
\text { Province }\end{array}$ & $\mathrm{Cu}( \pm \mathrm{Fe}, \mathrm{Au}, \mathrm{Ag}, \mathrm{Mo})$ skarn & $\mathrm{Cu}$ & Jiliaolu \\
\hline $\mathrm{J}$ & 51 & 10 & $\begin{array}{l}\text { Sanshandao, Shandong } \\
\text { Province }\end{array}$ & Granitoid-related Au vein & $\mathrm{Au}$ & Jiliaolu \\
\hline $\mathrm{J}$ & 51 & 11 & $\begin{array}{l}\text { Jiaojia, Shandong } \\
\text { Province }\end{array}$ & Granitoid-related Au vein & $\mathrm{Au}$ & Jiliaolu \\
\hline $\bar{J}$ & 51 & 12 & $\begin{array}{l}\text { Nanshu, Shandong } \\
\text { Province }\end{array}$ & Metamorphic graphite & Graphite & Jiliaojiao \\
\hline $\mathrm{J}$ & 51 & 2 & Fuxian, Liaoning Province & Diamond kimberlite & Diamond & East Liaoning \\
\hline $\mathrm{J}$ & 51 & 3 & Soyonpyong-do & Mafic-ultramafic related Ti-Fe (V) & $\mathrm{Fe}, \mathrm{Cr}, \mathrm{Ti}$ & Gyeonggi \\
\hline $\mathrm{J}$ & 51 & 4 & $\begin{array}{l}\text { Xiangkuang, Shandong } \\
\text { Province }\end{array}$ & $\mathrm{Zn}-\mathrm{Pb}(\mathrm{Ag}, \mathrm{Cu}, \mathrm{W})$ skarn & $\mathrm{Pb} \mathrm{Zn}$ & Jiliaolu \\
\hline $\mathrm{J}$ & 51 & 5 & $\begin{array}{l}\text { Linglong, Shandong } \\
\text { Province }\end{array}$ & Granitoid-related Au vein & $\mathrm{Au}$ & Jiliaolu \\
\hline $\mathrm{J}$ & 51 & 6 & Jiehe, Shandong Province & Granitoid-related Au vein & $\mathrm{Au}$ & Jiliaolu \\
\hline $\mathrm{J}$ & 51 & 7 & $\begin{array}{l}\text { Xincheng, Shandong } \\
\text { Province }\end{array}$ & Granitoid-related $\mathrm{Au}$ vein & $\mathrm{Au}$ & Jiliaolu \\
\hline $\mathrm{J}$ & 51 & 8 & $\begin{array}{l}\text { Jinqingding, Shandong } \\
\text { Province }\end{array}$ & Granitoid-related Au vein & $\mathrm{Au}$ & Jiliaolu \\
\hline $\mathrm{J}$ & 51 & 9 & $\begin{array}{l}\text { Shilipu, Shandong } \\
\text { Province }\end{array}$ & $\begin{array}{l}\text { Polymetallic }(\mathrm{Pb}, \mathrm{Zn} \pm \mathrm{Cu}, \mathrm{Ba}, \mathrm{Ag}, \mathrm{Au}) \text { volcanic-hosted } \\
\text { metasomatite }\end{array}$ & $\mathrm{Ag}$ & Jiliaolu \\
\hline $\mathrm{J}$ & 52 & 1 & Yangyang & Banded iron formation (BIF, Superior Fe) & $\mathrm{Fe}$ & Taebaegsan \\
\hline $\mathrm{J}$ & 52 & 10 & Chulam & Au skarn & $\mathrm{Au}, \mathrm{Ag}$ & Taebaegsan \\
\hline $\mathrm{J}$ & 52 & 11 & Uirim-Samwon & Polymetallic $(\mathrm{Pb}, \mathrm{Zn}, \mathrm{Ag})$ carbonate-hosted metasomatite & $\mathrm{Cu}, \mathrm{Pb}, \mathrm{Zn}$ & Taebaegsan \\
\hline $\mathrm{J}$ & 52 & 12 & Yomisan (Sinyemi) & $\mathrm{Zn}-\mathrm{Pb}(\mathrm{Ag}, \mathrm{Cu}, \mathrm{W})$ skarn & $\mathrm{Zn}, \mathrm{Fe}$ & Taebaegsan \\
\hline $\mathrm{J}$ & 52 & 13 & Soonkyong & Sn-W greisen, stockwork, and quartz vein & $\mathrm{Sn}$ & Taebaegsan \\
\hline $\mathrm{J}$ & 52 & 14 & Kumsan & W-Mo-Be greisen, stockwork, and quartz vein & W, Mo & Gyeongpuk \\
\hline $\mathrm{J}$ & 52 & 15 & Wangpiri & Sn-W greisen, stockwork, and quartz vein & $\mathrm{Sn}$ & Gyeongpuk \\
\hline $\mathrm{J}$ & 52 & 16 & Chilbo & $\mathrm{W} \pm \mathrm{Mo} \pm \mathrm{Be}$ skarn & $\mathrm{W}, \mathrm{Fe}$ & Gyeonggi \\
\hline $\mathrm{J}$ & 52 & 17 & Kumjang & Polymetallic $\mathrm{Pb}-\mathrm{Zn} \pm \mathrm{Cu}( \pm \mathrm{Ag}, \mathrm{Au})$ vein and stockwork & $\mathrm{Cu}, \mathrm{Pb}, \mathrm{Zn}$ & Gyeongpuk \\
\hline $\mathrm{J}$ & 52 & 18 & Eungok & Polymetallic $\mathrm{Pb}-\mathrm{Zn} \pm \mathrm{Cu}( \pm \mathrm{Ag}, \mathrm{Au})$ vein and stockwork & $\mathrm{Pb}, \mathrm{Zn}$ & Eungok \\
\hline $\mathrm{J}$ & 52 & 19 & Daejang & Fe-Zn skarn & $\mathrm{Fe}, \mathrm{Cu}$ & Gyeongpuk \\
\hline $\mathrm{J}$ & 52 & 2 & Kangwon & Fe skarn & $\mathrm{Fe}$ & Taebaegsan \\
\hline
\end{tabular}




\begin{tabular}{|c|c|c|c|c|c|c|}
\hline Row & Col. & No. & Deposit Name & Mineral Deposit Type & Major Metals & Metallogenic Belt \\
\hline $\mathrm{J}$ & 52 & 20 & Bupyoung & Volcanic-hosted Au-base-metal metasomatite & $\mathrm{Ag}, \mathrm{Pb}$ & Gyeonggi \\
\hline $\mathrm{J}$ & 52 & 21 & Oryu-dong & Metamorphic graphite & Graphite & Gyeonggi \\
\hline $\mathrm{J}$ & 52 & 22 & Youngdeog & Polymetallic $\mathrm{Pb}-\mathrm{Zn} \pm \mathrm{Cu}( \pm \mathrm{Ag}, \mathrm{Au})$ vein and stockwork & $\mathrm{Pb}, \mathrm{Zn}$ & Wolak \\
\hline $\mathrm{J}$ & 52 & 23 & Jesamuk & $\mathrm{Cu}( \pm \mathrm{Fe}, \mathrm{Au}, \mathrm{Ag}, \mathrm{Mo})$ skarn & $\mathrm{Cu}, \mathrm{Pb}, \mathrm{Zn}$ & Wolak \\
\hline $\mathrm{J}$ & 52 & 24 & Susan & Fe skarn & $\mathrm{Fe}, \mathrm{Mn}$ & Wolak \\
\hline $\mathrm{J}$ & 52 & 25 & Seojom & Au in shear zone and quartz vein & $\mathrm{Au}, \mathrm{Ag}$ & Taebaegsan \\
\hline $\mathrm{J}$ & 52 & 26 & Seosan & Banded iron formation (BIF, Superior Fe) & $\mathrm{Fe}, \mathrm{Cu}$ & Chungnam \\
\hline $\mathrm{J}$ & 52 & 27 & Yungchang 2 & $\mathrm{Ni}-\mathrm{Co}$ arsenide vein & $\mathrm{Co}, \mathrm{Ni}$ & Eungok \\
\hline $\mathrm{J}$ & 52 & 28 & Chilgok & Polymetallic $\mathrm{Pb}-\mathrm{Zn} \pm \mathrm{Cu}( \pm \mathrm{Ag}, \mathrm{Au})$ vein and stockwork & $\mathrm{Pb}, \mathrm{Zn}$ & Gyeongpuk \\
\hline $\mathrm{J}$ & 52 & 29 & Samkwang & Polymetaliic Ni vein & $\mathrm{Ni}$ & Gyeongpuk \\
\hline $\mathrm{J}$ & 52 & 3 & Hongcheon-Jaun & Polygenic REE-Fe-Nb deposit (Bayan-Obo type) & $\mathrm{Fe}, \mathrm{SrO}$ & Taebaegsan \\
\hline $\mathrm{J}$ & 52 & 30 & Kongju & Metamorphic graphite & Graphite & Chungnam \\
\hline $\mathrm{J}$ & 52 & 31 & Samgoe-Soryong & Clastic-sediment-hosted U & $\mathrm{U}$ & Chungnam \\
\hline $\mathrm{J}$ & 52 & 32 & Sangdong & $\mathrm{W} \pm \mathrm{Mo} \pm \mathrm{Be}$ skarn & $\mathrm{W}$ & Taebaegsan \\
\hline $\mathrm{J}$ & 52 & 4 & Susuk & Fe skarn & $\mathrm{Fe}$ & Taebaegsan \\
\hline $\mathrm{J}$ & 52 & 5 & Seongdong & Fe skarn & $\mathrm{Fe}$ & Gyeonggi \\
\hline $\mathrm{J}$ & 52 & 6 & Samchok & Banded iron formation (BIF, Superior Fe) & $\mathrm{Fe}$ & Taebaegsan \\
\hline $\mathrm{J}$ & 52 & 7 & Wondong & $\mathrm{W} \pm \mathrm{Mo} \pm \mathrm{Be}$ skarn & $\mathrm{W}, \mathrm{Fe}, \mathrm{Pb}$. & Taebaegsan \\
\hline $\mathrm{J}$ & 52 & 8 & Dongnam & Fe-Zn skarn & $\mathrm{Fe}, \mathrm{Mo}$ & Taebaegsan \\
\hline $\mathrm{J}$ & 52 & 9 & Gapyeong & Metamorphic graphite & Graphite & Gyeonggi \\
\hline $\mathrm{J}$ & 53 & 1 & Sennotani & Metamorphic graphite & Graphite & Inner Zone Southwest Japan \\
\hline $\mathrm{J}$ & 53 & 2 & Kamioka Mozumi & $\mathrm{Zn}-\mathrm{Pb}(\mathrm{Ag}, \mathrm{Cu}, \mathrm{W})$ skarn & $\mathrm{Zn}, \mathrm{Pb}, \mathrm{Ag}$ & Inner Zone Southwest Japan \\
\hline $\mathrm{J}$ & 53 & 3 & Koshimizu & Metamorphic graphite & Graphite & Inner Zone Southwest Japan \\
\hline $\mathrm{J}$ & 53 & 4 & Kamioka Tochibora & $\mathrm{Zn}-\mathrm{Pb}(\mathrm{Ag}, \mathrm{Cu}, \mathrm{W})$ skarn & $\mathrm{Zn}, \mathrm{Pb}, \mathrm{Ag}$ & Inner Zone Southwest Japan \\
\hline $\mathrm{J}$ & 53 & 5 & Amo & Metamorphic graphite & Graphite & Inner Zone Southwest Japan \\
\hline $\mathrm{J}$ & 53 & 6 & Hokuriku & Au-Ag epithermal vein & $\mathrm{Cu}, \mathrm{Zn}, \mathrm{Pb}, \mathrm{Ag}$ & Hokuriku-Sanin \\
\hline $\mathrm{J}$ & 53 & 7 & Hirase & Porphyry Mo $( \pm \mathrm{W}, \mathrm{Sn}, \mathrm{Bi})$ & Mo & Inner Zone Southwest Japan \\
\hline $\mathrm{J}$ & 53 & 8 & Bandojima & $\mathrm{Cu}( \pm \mathrm{Fe}, \mathrm{Au}, \mathrm{Ag}, \mathrm{Mo})$ skarn & $\mathrm{Pb}, \mathrm{Zn}, \mathrm{Cu}$ & Inner Zone Southwest Japan \\
\hline $\mathrm{J}$ & 54 & 1 & Taro & $\begin{array}{l}\text { Volcanogenic } \mathrm{Zn}-\mathrm{Pb}-\mathrm{Cu} \text { massive sulfide (Kuroko, Altai } \\
\text { type) }\end{array}$ & $\mathrm{Cu}, \mathrm{Zn}, \mathrm{Pb}$ & North Kitakami \\
\hline $\mathrm{J}$ & 54 & 10 & Unekura & $\mathrm{Au}-\mathrm{Ag}$ epithermal vein & $\mathrm{Cu}$ & Northeast Japan \\
\hline $\mathrm{J}$ & 54 & 11 & Oya & Granitoid-related $\mathrm{Au}$ vein & $\mathrm{Au}, \mathrm{Ag}$ & Kitakami \\
\hline $\mathrm{J}$ & 54 & 12 & Yoshino & $\begin{array}{l}\text { Volcanogenic } \mathrm{Zn}, \mathrm{Pb}, \mathrm{Cu} \text { massive sulfide (Kuroko, Altai } \\
\text { type) }\end{array}$ & $\mathrm{Cu}, \mathrm{Ag}$ & Northeast Japan \\
\hline $\mathrm{J}$ & 54 & 13 & Hosokura & Au-Ag epithermal vein & $\mathrm{Zn}, \mathrm{Pb}, \mathrm{Cu}$ & Northeast Japan \\
\hline $\mathrm{J}$ & 54 & 14 & Nagamatsu & $\mathrm{Au}-\mathrm{Ag}$ epithermal vein & $\mathrm{Cu}$ & Northeast Japan \\
\hline $\mathrm{J}$ & 54 & 15 & Koyama & $\mathrm{Au}-\mathrm{Ag}$ epithermal vein & $\mathrm{Au}, \mathrm{Ag}, \mathrm{Cu}$ & Northeast Japan \\
\hline $\mathrm{J}$ & 54 & 16 & Oizumi & $\mathrm{Au}-\mathrm{Ag}$ epithermal vein & $\mathrm{Zn}, \mathrm{Pb}, \mathrm{Cu}$ & Northeast Japan \\
\hline $\mathrm{J}$ & 54 & 17 & Zao & Sulfur-sulfide (S, FeS2) & $\mathrm{S}$ & Northeast Japan \\
\hline $\mathrm{J}$ & 54 & 18 & Asahi (Budo) & Polymetallic $\mathrm{Pb}-\mathrm{Zn} \pm \mathrm{Cu}( \pm \mathrm{Ag}, \mathrm{Au})$ vein and stockwork & $\mathrm{Zn}, \mathrm{Pb}$ & Northeast Japan \\
\hline $\mathrm{J}$ & 54 & 19 & Yamagata-Yoshino & $\begin{array}{l}\text { Volcanogenic } \mathrm{Zn}-\mathrm{Pb}-\mathrm{Cu} \text { massive sulfide (Kuroko, Altai } \\
\text { type) }\end{array}$ & $\mathrm{Cu}, \mathrm{Zn}$ & Northeast Japan \\
\hline $\mathrm{J}$ & 54 & 2 & Matsuo & Sulfur-sulfide $(\mathrm{S}, \mathrm{FeS} 2)$ & $\mathrm{S}$ & Northeast Japan \\
\hline $\mathrm{J}$ & 54 & 20 & Yatani & $\mathrm{Au}-\mathrm{Ag}$ epithermal vein & $\mathrm{Zn}, \mathrm{Pb}, \mathrm{Ag}$ & Northeast Japan \\
\hline $\mathrm{J}$ & 54 & 21 & Numajiri & Sulfur-sulfide (S, FeS2) & $\mathrm{S}$ & Northeast Japan \\
\hline $\mathrm{J}$ & 54 & 22 & Iide & $\mathrm{Zn}-\mathrm{Pb}(\mathrm{Ag}, \mathrm{Cu}, \mathrm{W})$ skarn & $\mathrm{Zn}, \mathrm{Pb}$ & Inner Zone Southwest Japan \\
\hline $\mathrm{J}$ & 54 & 23 & Takatama & Au-Ag epithermal vein & $\mathrm{Au}, \mathrm{Ag}$ & Northeast Japan \\
\hline $\mathrm{J}$ & 54 & 24 & Yaguki & $\mathrm{Cu}( \pm \mathrm{Fe}, \mathrm{Au}, \mathrm{Ag}, \mathrm{Mo})$ skarn & $\mathrm{Cu}, \mathrm{Fe}$ & Kitakami \\
\hline $\mathrm{J}$ & 54 & 25 & Sado & $\mathrm{Au}-\mathrm{Ag}$ epithermal vein & $\mathrm{Au}, \mathrm{Ag}, \mathrm{Cu}$ & Northeast Japan \\
\hline $\mathrm{J}$ & 54 & 26 & Yasou & $\mathrm{Au}-\mathrm{Ag}$ epithermal vein & $\mathrm{Cu}$ & Northeast Japan \\
\hline $\mathrm{J}$ & 54 & 27 & Hitachi & Volcanogenic $\mathrm{Zn}-\mathrm{Pb}-\mathrm{Cu}$ massive sulfide (Kuroko, Altai & $\mathrm{Cu}$ & Hitachi \\
\hline
\end{tabular}




\begin{tabular}{|c|c|c|c|c|c|c|}
\hline & & & & type) & & \\
\hline $\mathrm{J}$ & 54 & 28 & Nan'etsu & Polymetallic $\mathrm{Pb}-\mathrm{Zn} \pm \mathrm{Cu}( \pm \mathrm{Ag}, \mathrm{Au})$ vein and stockwork & $\mathrm{Zn}, \mathrm{Pb}$ & Inner Zone Southwest Japan \\
\hline $\mathrm{J}$ & 54 & 29 & Tochigi & $\mathrm{Au}-\mathrm{Ag}$ epithermal vein & $\mathrm{Cu}$ & Northeast Japan \\
\hline $\mathrm{J}$ & 54 & 3 & Yamada district & Volcanogenic-sedimentary Mn & $\mathrm{Mn}$ & North Kitakami \\
\hline $\mathrm{J}$ & 54 & 30 & Nebazawa & Au-Ag epithermal vein & $\mathrm{Ag}, \mathrm{Au}$ & Northeast Japan \\
\hline $\mathrm{J}$ & 54 & 31 & Takatori & W-Mo-Be greisen, stockwork, and quartz vein & $\mathrm{W}$ & Inner Zone Southwest Japan \\
\hline $\mathrm{J}$ & 54 & 32 & Ashio & Polymetallic $\mathrm{Pb}-\mathrm{Zn} \pm \mathrm{Cu}( \pm \mathrm{Ag}, \mathrm{Au})$ vein and stockwork & $\mathrm{Cu}, \mathrm{Zn}, \mathrm{Au}, \mathrm{Ag}$ & Northeast Japan \\
\hline $\mathrm{J}$ & 54 & 33 & Awano district & Volcanogenic-sedimentary Mn & $\mathrm{Mn}$ & Mino-Tamba-Chugoku \\
\hline $\mathrm{J}$ & 54 & 34 & Watarase district & Volcanogenic-sedimentary $\mathrm{Mn}$ & $\mathrm{Mn}$ & Mino-Tamba-Chugoku \\
\hline $\mathrm{J}$ & 54 & 35 & Gumma & Limonite from spring water & $\mathrm{Fe}$ & Northeast Japan \\
\hline $\mathrm{J}$ & 54 & 36 & Kusatsu-Shirane district & Sulfur-sulfide (S, FeS2) & $\mathrm{S}$ & Northeast Japan \\
\hline $\mathrm{J}$ & 54 & 37 & Yonago & Sulfur-sulfide (S, FeS2) & $\mathrm{S}$ & Northeast Japan \\
\hline $\mathrm{J}$ & 54 & 38 & Chichibu & $\mathrm{Zn}-\mathrm{Pb}( \pm \mathrm{Ag}, \mathrm{Cu}, \mathrm{W})$ skarn & $\begin{array}{l}\mathrm{Ag}, \mathrm{Au}, \mathrm{Zn}, \mathrm{Pb} \\
\mathrm{Fe}\end{array}$ & Outer Zone Southwest Japan \\
\hline $\mathrm{J}$ & 54 & 39 & Omine & $\mathrm{Cu}( \pm \mathrm{Fe}, \mathrm{Au}, \mathrm{Ag}, \mathrm{Mo})$ skarn & $\mathrm{Cu}, \mathrm{Au}, \mathrm{Ag}$ & Kitakami \\
\hline $\mathrm{J}$ & 54 & 4 & Ani & $\mathrm{Au}-\mathrm{Ag}$ epithermal vein & $\mathrm{Cu}, \mathrm{Au}, \mathrm{Ag}$ & Northeast Japan \\
\hline $\mathrm{J}$ & 54 & 5 & Kamaishi & $\mathrm{Cu}( \pm \mathrm{Fe}, \mathrm{Au}, \mathrm{Ag}, \mathrm{Mo})$ skarn & $\mathrm{Fe}, \mathrm{Cu}$ & Kitakami \\
\hline $\mathrm{J}$ & 54 & 6 & Arakawa & Au-Ag epithermal vein & $\mathrm{Cu}$ & Northeast Japan \\
\hline $\mathrm{J}$ & 54 & 7 & Akagane & $\mathrm{Cu}( \pm \mathrm{Fe}, \mathrm{Au}, \mathrm{Ag}, \mathrm{Mo})$ skarn & $\mathrm{Cu}, \mathrm{Fe}, \mathrm{Au}$ & Kitakami \\
\hline $\mathrm{J}$ & 54 & 8 & Tsuchihata & $\begin{array}{l}\text { Volcanogenic } \mathrm{Zn}-\mathrm{Pb}-\mathrm{Cu} \text { massive sulfide (Kuroko, Altai } \\
\text { type) }\end{array}$ & $\mathrm{Cu}$ & Northeast Japan \\
\hline $\mathrm{J}$ & 54 & 9 & Washiaimori & $\mathrm{Au}-\mathrm{Ag}$ epithermal vein & $\mathrm{Cu}, \mathrm{Ag}$ & Northeast Japan \\
\hline $\mathrm{K}$ & 47 & 1 & Khatansuudal & Clastic-sediment-hosted Sb-Au & $\mathrm{Au}$ & Tomortein Nuruu . \\
\hline $\mathrm{K}$ & 47 & 2 & Qiyishan, Inner Mongolia & $\mathrm{W} \pm \mathrm{Mo} \pm \mathrm{Be}$ skarn & $\mathrm{Rb}, \mathrm{W}, \mathrm{Sn}$ & Hartolgoi-sulinheer \\
\hline $\mathrm{K}$ & 47 & 3 & East Khatansuudal & Clastic-sediment-hosted Sb-Au & $\mathrm{Au}$ & Tomortein Nuruu \\
\hline $\mathrm{K}$ & 47 & 4 & Talynmeltes & Clastic-sediment-hosted Sb-Au & $\mathrm{Au}$ & Tomortein Nuruu \\
\hline K & 47 & 5 & $\begin{array}{l}\text { Liusashan, Inner } \\
\text { Mongolia }\end{array}$ & Porphyry Mo $( \pm \mathrm{W}, \mathrm{Sn}, \mathrm{Bi})$ & Mo & Hartolgoi-Sulinheer \\
\hline $\mathrm{K}$ & 47 & 6 & Guut hudag & Au-Ag epithermal vein & $\mathrm{Au}$ & Unassigned \\
\hline $\mathrm{K}$ & 48 & 1 & Shuteen & $\mathrm{Au}-\mathrm{Ag}$ epithermal vein & $\mathrm{Au}$ & Harmagtai-Hongoot-Oyut \\
\hline $\mathrm{K}$ & 48 & 10 & Huogeqi, Inner Mongolia & Sedimentary exhalative $\mathrm{Pb}-\mathrm{Zn}$ (SEDEX) & $\mathrm{Cu}, \mathrm{Pb}, \mathrm{Zn}$ & Langshan-Bayan Obo \\
\hline $\bar{K}$ & 48 & 12 & $\begin{array}{l}\text { Dongshengmiao, Inner } \\
\text { Mongolia }\end{array}$ & Sedimentary exhalative $\mathrm{Pb}-\mathrm{Zn}$ (SEDEX) & $\mathrm{Zn}, \mathrm{Pb}, \mathrm{Cu}$ & Langshan-Bayan Obo \\
\hline K & 48 & 13 & $\begin{array}{l}\text { Tanyaokou, Inner } \\
\text { Mongolia }\end{array}$ & Sedimentary exhalative $\mathrm{Pb}-\mathrm{Zn}$ (SEDEX) & $\mathrm{Zn} \mathrm{Cu}$ & Langshan-Bayan Obo \\
\hline $\mathrm{K}$ & 48 & 2 & Khan Bogd & Ta-Nb-REE alkaline metasomatite & $\mathrm{Nb}, \mathrm{REE}$ & Harmorit-Hanbogd-Lugiingol \\
\hline $\mathrm{K}$ & 48 & 3 & Oyu Tolgoi & Porphyry $\mathrm{Cu}( \pm \mathrm{Au})$ & $\mathrm{Cu}$ & Tsagaansuvarga \\
\hline $\mathrm{K}$ & 48 & 4 & Zuun Togoo Uul & Carbonate-hosted $\mathrm{Hg}$-Sb & $\mathrm{Sb}$ & Hartolgoi-Sulinheer \\
\hline K & 48 & 5 & Alag tolgoi & Granitoid-related $\mathrm{Au}$ vein & $\mathrm{Au}$ & Tsagaansuvarga \\
\hline $\mathrm{K}$ & 48 & 6 & Suhayt & Au-Ag epithermal vein & $\mathrm{Au}$ & Unassigned \\
\hline $\mathrm{K}$ & 48 & 7 & Khartolgoi & Polymetallic $\mathrm{Pb}-\mathrm{Zn} \pm \mathrm{Cu}( \pm \mathrm{Ag}, \mathrm{Au})$ vein and stockwork & $\mathrm{Pb}, \mathrm{Ag}, \mathrm{Sb}$ & Hartolgoi-Sulinheer \\
\hline $\mathrm{K}$ & 48 & 8 & Zurkh & Barite vein & $\mathrm{Ag}, \mathrm{Pb}$ & Unassigned \\
\hline K & 48 & 9 & Khar morit & Sn-W greisen, stockwork, and quartz vein & Sn, W & Harmorit-Hanbogd-Lugiingol \\
\hline $\mathrm{K}$ & 49 & 1 & $\begin{array}{l}\text { Wenduermiao, Inner } \\
\text { Mongolia }\end{array}$ & Volcanogenic-sedimentary Fe & $\mathrm{Fe}$ & Wunduermiao \\
\hline K & 49 & 10 & Khoit Barjin & Au-Ag epithermal vein & $\mathrm{Au}$ & Hartolgoi-Sulinheer \\
\hline $\mathrm{K}$ & 49 & 11 & $\begin{array}{l}\text { Saiyinwusu, Inner } \\
\text { Mongolia }\end{array}$ & Granitoid-related Au vein & $\mathrm{Au}$ & Wulashan-Zhangbei \\
\hline $\mathrm{K}$ & 49 & 12 & Sulinheer group & Podiform chromite & $\mathrm{Cr}$ & Sulinheer \\
\hline K & 49 & 13 & $\begin{array}{l}\text { Bayan Obo, Inner } \\
\text { Mongolia }\end{array}$ & Polygenic REE-Fe-Nb deposit (Bayan-Obo type) & REE, $\mathrm{Fe}, \mathrm{Nb}$ & Langshan-Bayan Obo \\
\hline
\end{tabular}




\begin{tabular}{|c|c|c|c|c|c|c|}
\hline Row & Col. & No. & Deposit Name & Mineral Deposit Type & Major Metals & Metallogenic Belt \\
\hline K & 49 & 14 & $\begin{array}{l}\text { Sanheming, Inner } \\
\text { Mongolia }\end{array}$ & Banded iron formation (BIF, Algoma Fe) & $\mathrm{Fe}$ & Yinshan \\
\hline K & 49 & 15 & $\begin{array}{l}\text { Donghuofuang, Inner } \\
\text { Mongolia }\end{array}$ & Alkaline complex-hosted $\mathrm{Au}$ & $\mathrm{Au}$ & Wulashan-Zhangbei \\
\hline K & 49 & 16 & $\begin{array}{l}\text { Houshihua, Inner } \\
\text { Mongolia }\end{array}$ & Granitoid-related Au vein & $\mathrm{Au}$ & Wulashan-Zhangbei \\
\hline K & 49 & 17 & $\begin{array}{l}\text { Jiashengpan, Inner } \\
\text { Mongolia }\end{array}$ & Sedimentary exhalative Pb-Zn (SEDEX) & $\mathrm{Pb} \mathrm{Zn}$ & Langshan-Bayan Obo \\
\hline K & 49 & 18 & $\begin{array}{l}\text { Hadamengou, Inner } \\
\text { Mongolia }\end{array}$ & Au potassium metasomatite (Kuranakh type) & $\mathrm{Au}$ & Wulashan-Zhangbei \\
\hline K & 49 & 19 & $\begin{array}{l}\text { Wulashan, Baotou City, } \\
\text { Inner Mongolia }\end{array}$ & Au potassium metasomatite (Kuranakh type) & $\mathrm{Au}$ & Wulashan-Zhangbei \\
\hline K & 49 & 2 & $\begin{array}{l}\text { Sumochaganaobao, Inner } \\
\text { Mongolia }\end{array}$ & Hydrothermal-sedimentary fluorite & fluorite & Sumochaganaobo \\
\hline K & 49 & 3 & $\begin{array}{l}\text { Bieluwutu, Inner } \\
\text { Mongolia }\end{array}$ & $\begin{array}{l}\text { Volcanogenic-hydrothermal-sedimentary massive sulfide } \\
\quad \mathrm{Pb}-\mathrm{Zn}( \pm \mathrm{Cu})\end{array}$ & $\mathrm{Cu}$ & Bieluwutu \\
\hline K & 49 & 4 & Aobaotu, Inner Mongolia & Hydrothermal-sedimentary fluorite & Fluorite & Sumochaganaobo \\
\hline K & 49 & 5 & $\begin{array}{l}\text { Hadamiao, Inner } \\
\text { Mongolia }\end{array}$ & Granitoid-related $\mathrm{Au}$ vein & $\mathrm{Au}$ & Wulashan-Zhangbei \\
\hline $\mathrm{K}$ & 49 & 6 & Tsagaan Suvarga & Porphyry $\mathrm{Cu}-\mathrm{Mo}( \pm \mathrm{Au}, \mathrm{Ag})$ & $\mathrm{Cu}, \mathrm{Mo}$ & Tsagaansuvarga \\
\hline K & 49 & 7 & $\begin{array}{l}\text { Bainaimiao, Inner } \\
\text { Mongolia }\end{array}$ & Porphyry $\mathrm{Cu}-\mathrm{Mo}( \pm \mathrm{Au}, \mathrm{Ag})$ & $\mathrm{Cu}$ & Bainaimiao \\
\hline K & 49 & 8 & Horgo uul & Sedimentary celestite & $\mathrm{Sr}$ & Govi-Tamsag \\
\hline $\mathrm{K}$ & 49 & 9 & Lugingol & $\mathrm{REE}( \pm \mathrm{Ta}, \mathrm{Nb}, \mathrm{Fe})$ carbonatite & REE & Harmorit-Hanbogd-Lugiingol \\
\hline K & 50 & 1 & Dajing,Inner Mongolia & Polymetallic $\mathrm{Pb}-\mathrm{Zn} \pm \mathrm{Cu}( \pm \mathrm{Ag}, \mathrm{Au})$ vein and stockwork & $\mathrm{Ag}, \mathrm{Sn}$ & Daxinganling \\
\hline K & 50 & 10 & $\begin{array}{l}\text { Honghuagou, Inner } \\
\text { Mongolia }\end{array}$ & Granitoid-related $\mathrm{Au}$ vein & $\mathrm{Au}$ & Yanshan \\
\hline K & 50 & 11 & $\begin{array}{l}\text { Xiaotazhigou, Liaoning } \\
\text { Province }\end{array}$ & Granitoid-related Au vein & $\mathrm{Au}$ & Yanshan \\
\hline K & 50 & 12 & $\begin{array}{l}\text { Anjiayingzhi, Inner } \\
\text { Mongolia }\end{array}$ & Granitoid-related Au vein & $\mathrm{Au}$ & Yanshan \\
\hline K & 50 & 13 & $\begin{array}{l}\text { Reshui, Ningcheng, Inner } \\
\text { Mongolia }\end{array}$ & Granitoid-related Au vein & $\mathrm{Au}$ & Yanshan \\
\hline K & 50 & 14 & $\begin{array}{l}\text { Xiaojiayingzi, Liaoning } \\
\text { Province }\end{array}$ & $\mathrm{W} \pm \mathrm{Mo} \pm \mathrm{Be}$ skarn & Mo & Yanshan \\
\hline $\bar{K}$ & 50 & 15 & $\begin{array}{l}\text { Xiaokouhuaying, } \\
\text { Weichang, Hebei } \\
\text { Province }\end{array}$ & Au-Ag epithermal vein & $\mathrm{Ag}$ & Yanshan \\
\hline K & 50 & 16 & $\begin{array}{l}\text { Taipinggou, Liaoning } \\
\text { Province }\end{array}$ & Chemical-sedimentary Fe-Mn & $\mathrm{Mn}$ & Yanliao-2 \\
\hline $\bar{K}$ & 50 & 17 & $\begin{array}{l}\text { Xiaosigou, Pingquan } \\
\text { County, Hebei Province }\end{array}$ & Porphyry $\mathrm{Cu}-\mathrm{Mo}( \pm \mathrm{Au}, \mathrm{Ag})$ & $\mathrm{Cu}$ & Yanshan \\
\hline $\mathrm{K}$ & 50 & 18 & Gaositai, Hebei Province & Zoned mafic-ultramafic Cr-PGE & $\mathrm{Cr}$ & Damiao \\
\hline K & 50 & 19 & $\begin{array}{l}\text { Baizhangzhi, Liaoning } \\
\text { Province }\end{array}$ & Granitoid-related Au vein & $\mathrm{Au}$ & Yashan \\
\hline $\mathrm{K}$ & 50 & 2 & Anle, Inner Mongolia & Polymetallic $\mathrm{Pb}-\mathrm{Zn} \pm \mathrm{Cu}( \pm \mathrm{Ag}, \mathrm{Au})$ vein and stockwork & Sn & Daxinganling \\
\hline K & 50 & 20 & $\begin{array}{l}\text { Niujuan, Fengning, Hebei } \\
\text { Province }\end{array}$ & Au-Ag epithermal vein & $\mathrm{Ag}$ & Yanshan \\
\hline $\mathrm{K}$ & 50 & 21 & Damiao, Hebei Province & Mafic-ultramafic related Ti-Fe (V) & $\mathrm{Fe}, \mathrm{Ti}, \mathrm{V}$ & Damiao \\
\hline K & 50 & 22 & $\begin{array}{l}\text { Luoguozigou, Hebei } \\
\text { Province }\end{array}$ & Mafic-ultramafic related Ti-Fe (V) & $\mathrm{P}$ & Luoguozigou \\
\hline $\mathrm{K}$ & 50 & 23 & Xiazhangzhi, Hebei & Au-Ag epithermal vein & $\mathrm{Au}$ & Yanshan \\
\hline
\end{tabular}




\begin{tabular}{|c|c|c|c|c|c|c|}
\hline & & & Province & & & \\
\hline K & 50 & 24 & $\begin{array}{l}\text { Jiaodingshan, Chengde, } \\
\text { Hebei Province }\end{array}$ & Polymetallic $\mathrm{Pb}-\mathrm{Zn} \pm \mathrm{Cu}( \pm \mathrm{Ag}, \mathrm{Au})$ vein and stockwork & $\mathrm{Pb}$ & Yanshan \\
\hline $\mathrm{K}$ & 50 & 25 & Heishan, Hebei Province & Mafic-ultramafic related Ti-Fe (V) & $\mathrm{TiFe}$ & Damiao \\
\hline $\mathrm{K}$ & 50 & 26 & $\begin{array}{l}\text { Yingfang, Fengning, } \\
\text { Hebei Province }\end{array}$ & Polymetallic $\mathrm{Pb}-\mathrm{Zn} \pm \mathrm{Cu}( \pm \mathrm{Ag}, \mathrm{Au})$ vein and stockwork & $\mathrm{Ag}$ & Yanshan \\
\hline $\mathrm{K}$ & 50 & 27 & Yu'erya, Hebei Province & Granitoid-related Au vein & $\mathrm{Au}$ & Yanshan \\
\hline K & 50 & 28 & $\begin{array}{l}\text { Qinglonghe, Qinglong } \\
\text { County, Hebei Province }\end{array}$ & Clastic-sediment-hosted Sb-Au & $\mathrm{Au}$ & Qinglong \\
\hline $\mathrm{K}$ & 50 & 29 & Miaogou, Hebei Province & Banded iron formation (BIF, Algoma Fe) & $\mathrm{Fe}$ & Qinglong \\
\hline K & 50 & 3 & $\begin{array}{l}\text { Huanggan, Inner } \\
\text { Mongolia }\end{array}$ & Sn skarn & $\mathrm{Sn}$ & Daxinganling \\
\hline $\mathrm{K}$ & 50 & 30 & $\begin{array}{l}\text { Zhalanzhangzhi, Hebei } \\
\text { Province }\end{array}$ & Banded iron formation (BIF, Algoma Fe) & $\mathrm{Fe}$ & Qinglong \\
\hline $\mathrm{K}$ & 50 & 31 & $\begin{array}{l}\text { Guantangzhi, Hebei } \\
\text { Province }\end{array}$ & Sedimentary exhalative $\mathrm{Pb}-\mathrm{Zn}$ (SEDEX) & Pyrite & Yanliao-2 \\
\hline K & 50 & 32 & $\begin{array}{l}\text { Niuxinshan, Hebei } \\
\text { Province }\end{array}$ & Granitoid-related Au vein & $\mathrm{Au}$ & Yanshan \\
\hline K & 50 & 33 & $\begin{array}{l}\text { Wanquansi, Chicheng, } \\
\text { Hebei Province }\end{array}$ & $\begin{array}{l}\text { Polymetallic }(\mathrm{Pb}, \mathrm{Zn} \pm \mathrm{Cu}, \mathrm{Ba}, \mathrm{Ag}, \mathrm{Au}) \text { volcanic-hosted } \\
\text { metasomatite }\end{array}$ & $\mathrm{Ag}$ & Yanshan \\
\hline $\mathrm{K}$ & 50 & 34 & $\begin{array}{l}\text { Jinchangyu, Hebei } \\
\text { Province }\end{array}$ & $\mathrm{Au}$ in shear zone and quartz vein & $\mathrm{Au}$ & Jidong \\
\hline K & 50 & 35 & $\begin{array}{l}\text { Shouwangfen, Hebei } \\
\text { Province }\end{array}$ & $\mathrm{Cu}( \pm \mathrm{Fe}, \mathrm{Au}, \mathrm{Ag}, \mathrm{Mo})$ skarn & $\mathrm{Cu}$ & Yanshan \\
\hline K & 50 & 36 & $\begin{array}{l}\text { Caijiaying, Hebei } \\
\text { Province }\end{array}$ & Polymetallic $\mathrm{Pb}-\mathrm{Zn} \pm \mathrm{Cu}( \pm \mathrm{Ag}, \mathrm{Au})$ vein and stockwork & $\mathrm{Pb}, \mathrm{Zn}$ & Yanshan \\
\hline K & 50 & 37 & $\begin{array}{l}\text { Gaobanhe, Hebei } \\
\text { Province }\end{array}$ & Sedimentary exhalative $\mathrm{Pb}-\mathrm{Zn}$ (SEDEX) & $\mathrm{Pb}, \mathrm{Zn}$ & Yanliao-2 \\
\hline $\mathrm{K}$ & 50 & 38 & $\begin{array}{l}\text { Naobaogou, } \\
\text { Wulateqianqi, Inner } \\
\text { Mongolia }\end{array}$ & Alkaline complex-hosted $\mathrm{Au}$ & $\mathrm{Au}$ & Wulashan-Zhangbei \\
\hline $\mathrm{K}$ & 50 & 39 & $\begin{array}{l}\text { Shuichang, Hebei } \\
\text { Province }\end{array}$ & Banded iron formation (BIF, Algoma Fe) & $\mathrm{Fe}$ & Jidong \\
\hline K & 50 & 4 & $\begin{array}{l}\text { Aobaoshan,Inner } \\
\text { Mongolia }\end{array}$ & $\mathrm{Zn}-\mathrm{Pb}(\mathrm{Ag}, \mathrm{Cu}, \mathrm{W})$ skarn & $\mathrm{Pb}, \mathrm{Zn}$ & Daxinganling \\
\hline $\mathrm{K}$ & 50 & 40 & $\begin{array}{l}\text { Qingyanggou, Hebei } \\
\text { Province }\end{array}$ & Polymetallic $\mathrm{Pb}-\mathrm{Zn} \pm \mathrm{Cu}( \pm \mathrm{Ag}, \mathrm{Au})$ vein and stockwork & $\mathrm{Ag}$ & Yanshan \\
\hline $\mathrm{K}$ & 50 & 41 & Fengjiayu, Miyun, Beijing & Banded iron formation (BIF, Algoma Fe) & $\mathrm{Fe}$ & Jidong \\
\hline K & 50 & 42 & $\begin{array}{l}\text { Mengjiagou, Hebei } \\
\text { Province }\end{array}$ & Banded iron formation (BIF, Algoma Fe) & $\mathrm{Fe}$ & Jidong \\
\hline $\mathrm{K}$ & 50 & 43 & Donjiagou, Beijing & Porphyry $\mathrm{Cu}-\mathrm{Mo}( \pm \mathrm{Au}, \mathrm{Ag})$ & Mo & Yanshan \\
\hline $\mathrm{K}$ & 50 & 44 & $\begin{array}{l}\text { Shuijingtun, Chongli } \\
\text { County, Hebei Province }\end{array}$ & Granitoid-related Au vein & $\mathrm{Au}$ & Yanshan \\
\hline $\mathrm{K}$ & 50 & 45 & Pangjiapu, Hebei Province & Chemical-sedimentary Fe-Mn & $\mathrm{Fe}$ & Yanliao-1 \\
\hline $\mathrm{K}$ & 50 & 46 & $\begin{array}{l}\text { Zhongxinchun, Beijing } \\
\text { District }\end{array}$ & Chemical-sedimentary Fe-Mn & $\mathrm{B}, \mathrm{Mn}$ & Yanliao-2 \\
\hline K & 50 & 47 & $\begin{array}{l}\text { Xiaoyingpan, Hebei } \\
\text { Province }\end{array}$ & Alkaline complex-hosted $\mathrm{Au}$ & $\mathrm{Au}$ & Wulashan-Zhangbei \\
\hline $\mathrm{K}$ & 50 & 48 & Dazhuangke, Beijing & Porphyry Mo $( \pm \mathrm{W}, \mathrm{Sn}, \mathrm{Bi})$ & Mo & Yanshan \\
\hline $\mathrm{K}$ & 50 & 49 & $\begin{array}{l}\text { Yantongshan, Hebei } \\
\text { Province }\end{array}$ & Chemical-sedimentary Fe-Mn & $\mathrm{Fe}$ & Yanliao-1 \\
\hline $\mathrm{K}$ & 50 & 5 & Xiaoyingzi, Inner & $\mathrm{Zn}-\mathrm{Pb}(\mathrm{Ag}, \mathrm{Cu}, \mathrm{W})$ skarn & $\mathrm{Pb}, \mathrm{Zn}$ & Daxinganling \\
\hline
\end{tabular}




\begin{tabular}{|c|c|c|c|c|c|c|}
\hline & & & Mongolia & & & \\
\hline $\mathrm{K}$ & 50 & 50 & Fanshan, Hebei Province & Magmatic and metasomatic apatite & $\mathrm{P}$ & Fanshan \\
\hline $\mathrm{K}$ & 50 & 51 & Xinghe, Inner Mongolia & Metamorphic graphite & Graphite & Yanbei \\
\hline K & 50 & 52 & $\begin{array}{l}\text { Gongdianzhi (Qian'an), } \\
\text { Hebei Province }\end{array}$ & Banded iron formation (BIF, Algoma Fe) & $\mathrm{Fe}$ & Jidong \\
\hline $\mathrm{K}$ & 50 & 53 & Dongshiuchang, Tiejin & Chemical-sedimentary Fe-Mn & $\mathrm{B}, \mathrm{Mn}$ & Yanliao-2 \\
\hline K & 50 & 54 & $\begin{array}{l}\text { Shirengou, Hebei } \\
\text { Province }\end{array}$ & Banded iron formation (BIF, Algoma Fe) & $\mathrm{Fe}$ & Jidong \\
\hline K & 50 & 55 & Guzhigou, Hebei Province & $\begin{array}{l}\text { Polymetallic }(\mathrm{Pb}, \mathrm{Zn} \pm \mathrm{Cu}, \mathrm{Ba}, \mathrm{Ag}, \mathrm{Au}) \text { volcanic-hosted } \\
\text { metasomatite }\end{array}$ & $\mathrm{Ag}$ & Yanshan \\
\hline $\mathrm{K}$ & 50 & 56 & Dongping, Hebei Province & Alkaline complex-hosted $\mathrm{Au}$ & $\mathrm{Au}$ & Wulashan-Zhangbei \\
\hline K & 50 & 57 & $\begin{array}{l}\text { Jinjiazhuang, Hebei } \\
\text { Province }\end{array}$ & Granitoid-related $\mathrm{Au}$ vein & $\mathrm{Au}$ & Yanshan \\
\hline $\mathrm{K}$ & 50 & 58 & Shachang, Miyun, Beijing & Banded iron formation (BIF, Algoma Fe) & $\mathrm{Fe}$ & Jidong \\
\hline K & 50 & 59 & $\begin{array}{l}\text { Hougou, Chicheng, Hebei } \\
\text { Province }\end{array}$ & Alkaline complex-hosted $\mathrm{Au}$ & $\mathrm{Au}$ & Wulashan-Zhangbei \\
\hline K & 50 & 6 & Dongzi,Inner Mongolia & $\begin{array}{l}\text { Polymetallic }(\mathrm{Pb}, \mathrm{Zn} \pm \mathrm{Cu}, \mathrm{Ba}, \mathrm{Ag}, \mathrm{Au}) \text { volcanic-hosted } \\
\text { metasomatite }\end{array}$ & $\mathrm{Pb}, \mathrm{Zn}$ & Daxinganling \\
\hline $\mathrm{K}$ & 50 & 7 & Guandi, Inner Mongolia & Au-Ag epithermal vein & $\mathrm{Ag}$ & Daxinganling \\
\hline K & 50 & 8 & $\begin{array}{l}\text { Zhuanshanzi, Inner } \\
\text { Mongolia }\end{array}$ & Granitoid-related Au vein & $\mathrm{Au}$ & Yanshan \\
\hline $\bar{K}$ & 50 & 9 & $\begin{array}{l}\text { Shaoguoyingzhi, Jianping } \\
\text { County, Liaoning } \\
\text { Province }\end{array}$ & Granitoid-related Au vein & $\mathrm{Au}$ & Yanshan \\
\hline K & 51 & 1 & $\begin{array}{l}\text { Fangniugou, Jilin } \\
\text { Province }\end{array}$ & $\begin{array}{l}\text { Volcanogenic-hydrothermal-sedimentary massive sulfide } \\
\quad \mathrm{Pb}-\mathrm{Zn}( \pm \mathrm{Cu})\end{array}$ & $\mathrm{Zn}$ & Fangniugou \\
\hline K & 51 & 10 & $\begin{array}{l}\text { Hongtoushan, Liaoning } \\
\text { Province }\end{array}$ & $\begin{array}{l}\text { Volcanogenic } \mathrm{Zn}-\mathrm{Pb}-\mathrm{Cu} \text { massive sulfide (Kuroko, Altai } \\
\text { type) }\end{array}$ & $\mathrm{Cu}, \mathrm{Zn}$ & Liaoji \\
\hline $\mathrm{K}$ & 51 & 11 & Zihe, Liaoning Province & Carbonate-hosted $\mathrm{Pb}-\mathrm{Zn}$ (Mississippi valley type) & $\mathrm{Pb}, \mathrm{Zn}$ & Fanhe \\
\hline$\overline{\mathrm{K}}$ & 51 & 12 & $\begin{array}{l}\text { Wangjiadagou, Liaoning } \\
\text { Province }\end{array}$ & Granitoid-related Au vein & $\mathrm{Au}$ & Liaoji \\
\hline $\mathrm{K}$ & 51 & 13 & Zhengcha, Jilin Province & $\mathrm{Zn}-\mathrm{Pb}(\mathrm{Ag}, \mathrm{Cu}, \mathrm{W})$ skarn & $\mathrm{Pb}, \mathrm{Zn}$ & Liaoji \\
\hline K & 51 & 14 & $\begin{array}{l}\text { Huanren, Liaonig } \\
\text { Province }\end{array}$ & $\mathrm{Zn}-\mathrm{Pb}(\mathrm{Ag}, \mathrm{Cu}, \mathrm{W})$ skarn & $\mathrm{Pb}, \mathrm{Zn}$ & Jiliaolu \\
\hline $\mathrm{K}$ & 51 & 15 & $\begin{array}{l}\text { Yangmugan, Liaoning } \\
\text { Province }\end{array}$ & Sedimentary-metamorphic borate & B & Jiliaojiao \\
\hline K & 51 & 16 & $\begin{array}{l}\text { Waitoushan, Liaoning } \\
\text { Province }\end{array}$ & Banded iron formation (BIF, Algoma Fe) & $\mathrm{Fe}$ & Liaoji \\
\hline $\bar{K}$ & 51 & 17 & $\begin{array}{l}\text { Nanfen, Liaoning } \\
\text { Province }\end{array}$ & Banded iron formation (BIF, Algoma Fe) & $\mathrm{Fe}$ & Liaoji \\
\hline K & 51 & 18 & $\begin{array}{l}\text { Rongguan, Liaoning } \\
\text { Province }\end{array}$ & Evaporate sedimentary gypsum & Gypsum & Hunjuang-Taizihe \\
\hline $\bar{K}$ & 51 & 19 & $\begin{array}{l}\text { Zhuanmiao, Liaoning } \\
\text { Province }\end{array}$ & Sedimentary-metamorphic borate & B & Jiliaojiao \\
\hline $\mathrm{K}$ & 51 & 2 & Sanmen, Jilin Province & $\begin{array}{l}\text { Polymetallic }(\mathrm{Pb}, \mathrm{Zn} \pm \mathrm{Cu}, \mathrm{Ba}, \mathrm{Ag}, \mathrm{Au}) \text { volcanic-hosted } \\
\text { metasomatite }\end{array}$ & $\mathrm{Ag}$ & North Jilin \\
\hline K & 51 & 20 & $\begin{array}{l}\text { Beidacheng, Aohan, Inner } \\
\text { Mongolia }\end{array}$ & Au-Ag epithermal vein & $\mathrm{Au}$ & Yanshan \\
\hline $\mathrm{K}$ & 51 & 21 & Baoguosi, Liaoning & Banded iron formation (BIF, Algoma Fe) & $\mathrm{Fe}$ & Liaoxi \\
\hline K & 51 & 22 & $\begin{array}{l}\text { Gongchangling, Anshan, } \\
\text { Liaoning Province }\end{array}$ & Banded iron formation (BIF, Algoma Fe) & $\mathrm{Fe}$ & Liaoji \\
\hline $\mathrm{K}$ & 51 & 23 & Daheishan 1, Aohan, & Granitoid-related Au vein & $\mathrm{Au}$ & Yanshan \\
\hline
\end{tabular}




\begin{tabular}{|c|c|c|c|c|c|c|}
\hline Row & Col. & No. & Deposit Name & Mineral Deposit Type & Major Metals & Metallogenic Belt \\
\hline & & & Inner Mongolia & & & \\
\hline K & 51 & 24 & $\begin{array}{l}\text { Jinchanggouliang, Inner } \\
\text { Mongolia }\end{array}$ & Granitoid-related Au vein & $\mathrm{Au}$ & Yanshan \\
\hline K & 51 & 25 & $\begin{array}{l}\text { Qidashan, Anshan, } \\
\text { Liaoning Province }\end{array}$ & Banded iron formation (BIF, Algoma Fe) & $\mathrm{Fe}$ & Liaoji \\
\hline $\bar{K}$ & 51 & 26 & $\begin{array}{l}\text { Erdaogou, Liaoning } \\
\text { Province }\end{array}$ & Au-Ag epithermal vein & $\mathrm{Au}$ & Yanshan \\
\hline K & 51 & 27 & $\begin{array}{l}\text { Yingtaoyuan, Anshan, } \\
\text { Liaoning Province }\end{array}$ & Banded iron formation (BIF, Algoma Fe) & $\mathrm{Fe}$ & Liaoji \\
\hline K & 51 & 28 & $\begin{array}{l}\text { Zhangjiagou, Liaoning } \\
\text { Province }\end{array}$ & $\begin{array}{l}\text { Volcanogenic-hydrothermal-sedimentary massive sulfide } \\
\quad \mathrm{Pb}-\mathrm{Zn}( \pm \mathrm{Cu})\end{array}$ & Pyrrhotite & Jiliaojiao \\
\hline K & 51 & 29 & $\begin{array}{l}\text { Wengquangou, Liaoning } \\
\text { Province }\end{array}$ & Sedimentary-metamorphic borate & $\mathrm{B}$ & Jiliaojiao \\
\hline K & 51 & 3 & $\begin{array}{l}\text { Xiangluwanzhi, Jilin } \\
\text { Province }\end{array}$ & Au-Ag epithermal vein & $\mathrm{Au}$ & Liaoji \\
\hline $\bar{K}$ & 51 & 30 & $\begin{array}{l}\text { Baiyunshan, Liaoning } \\
\text { Province }\end{array}$ & $\mathrm{Au}$ in shear zone and quartz vein & $\mathrm{Au}$ & Jiliaojiao \\
\hline K & 51 & 31 & $\begin{array}{l}\text { Qingchengzi, Liaoning } \\
\text { Province }\end{array}$ & Korean $\mathrm{Pb}-\mathrm{Zn}$ massive sulfide & $\mathrm{Pb}, \mathrm{Zn}$ & Jiliaojiao \\
\hline K & 51 & 32 & $\begin{array}{l}\text { Fanjiapuzi, Liaoning } \\
\text { Province }\end{array}$ & Talc (magnesite) replacement & Talc & Jiliaojiao \\
\hline $\bar{K}$ & 51 & 33 & $\begin{array}{l}\text { Xiafangshen, Liaoning } \\
\text { Province }\end{array}$ & Sedimentary-metamorphic magnesite & Magnesite & Jiliaojiao \\
\hline K & 51 & 34 & $\begin{array}{l}\text { Wulong, Liaoning } \\
\text { Province }\end{array}$ & Granitoid-related Au vein & $\mathrm{Au}$ & Jiliaolu \\
\hline $\mathrm{K}$ & 51 & 35 & $\begin{array}{l}\text { Sidaogou, Liaoning } \\
\text { Province }\end{array}$ & Granitoid-related Au vein & $\mathrm{Au}$ & Jiliaolu \\
\hline $\mathrm{K}$ & 51 & 36 & $\begin{array}{l}\text { Houxianyu, Liaoning } \\
\text { Province }\end{array}$ & Sedimentary-metamorphic borate & $\mathrm{B}$ & Jiliaojiao \\
\hline K & 51 & 37 & $\begin{array}{l}\text { Xiaoshengshuisi, } \\
\text { Liaoning Province }\end{array}$ & Sedimentary-metamorphic magnesite & Magnesite & Jiliaojiao \\
\hline K & 51 & 38 & $\begin{array}{l}\text { Paishanlou, Liaoning } \\
\text { Province }\end{array}$ & $\mathrm{Au}$ in shear zone and quartz vein & $\mathrm{Au}$ & Liaoxi \\
\hline K & 51 & 39 & $\begin{array}{l}\text { Xiuyuan 1, Liaoning } \\
\text { Province }\end{array}$ & Granitoid-related Au vein & $\mathrm{Au}$ & Jiliaolu \\
\hline K & 51 & 4 & $\begin{array}{l}\text { Nanlongwangmiao, } \\
\text { Liaoning Province }\end{array}$ & $\mathrm{Au}$ in shear zone and quartz vein & $\mathrm{Au}$ & Liaoji \\
\hline K & 51 & 40 & $\begin{array}{l}\text { Xiuyuan 2, Liaoning } \\
\text { Province }\end{array}$ & Polymetallic $\mathrm{Pb}-\mathrm{Zn} \pm \mathrm{Cu}( \pm \mathrm{Ag}, \mathrm{Au})$ vein and stockwork & $\mathrm{Pb}, \mathrm{Zn}$ & Jiliaolu \\
\hline $\mathrm{K}$ & 51 & 41 & $\begin{array}{l}\text { Lanjiagou, Liaoning } \\
\text { Province }\end{array}$ & Porphyry Mo ( $\pm \mathrm{W}, \mathrm{Sn}, \mathrm{Bi})$ & Mo & Yanshan \\
\hline $\mathrm{K}$ & 51 & 42 & $\begin{array}{l}\text { Chaoyang, Liaoning } \\
\text { Province }\end{array}$ & Carbonate-hosted asbestos & Asbestos & Yanliao-2 \\
\hline $\mathrm{K}$ & 51 & 43 & $\begin{array}{l}\text { Maoling, Liaoning } \\
\text { Province }\end{array}$ & $\mathrm{Au}$ in shear zone and quartz vein & $\mathrm{Au}$ & Jiliaojiao \\
\hline $\mathrm{K}$ & 51 & 44 & Liutun, Liaoning Province & Granitoid-related $\mathrm{Au}$ vein & $\mathrm{Au}$ & Yanshan \\
\hline K & 51 & 45 & $\begin{array}{l}\text { Wafangzi, Liaoning } \\
\text { Province }\end{array}$ & Chemical-sedimentary Fe-Mn & $\mathrm{Mn}$ & Yanliao-2 \\
\hline K & 51 & 46 & $\begin{array}{l}\text { Yangjiazhangzi, Liaoning } \\
\text { Province }\end{array}$ & $\mathrm{W} \pm \mathrm{Mo} \pm$ Be skarn & Mo & Yanshan \\
\hline K & 51 & 47 & $\begin{array}{l}\text { Gadagou, Liaoning } \\
\text { Province }\end{array}$ & Granitoid-related Au vein & $\mathrm{Au}$ & Yanshan \\
\hline
\end{tabular}




\begin{tabular}{|c|c|c|c|c|c|c|}
\hline Row & Col. & No. & Deposit Name & Mineral Deposit Type & Major Metals & Metallogenic Belt \\
\hline K & 51 & 48 & $\begin{array}{l}\text { Bajiazi, Liaoning } \\
\text { Province }\end{array}$ & $\mathrm{Zn}-\mathrm{Pb}(\mathrm{Ag}, \mathrm{Cu}, \mathrm{W})$ skarn & $\mathrm{Pb}, \mathrm{Zn}$ & Yanshan \\
\hline $\mathrm{K}$ & 51 & 5 & $\begin{array}{l}\text { Dahuanggou, Jilin } \\
\text { Province }\end{array}$ & $\begin{array}{l}\text { Volcanogenic } \mathrm{Zn}-\mathrm{Pb}-\mathrm{Cu} \text { massive sulfide (Kuroko, Altai } \\
\text { type) }\end{array}$ & Pyrite & Liaoji \\
\hline K & 51 & 6 & $\begin{array}{l}\text { Chaihe, Liaoning } \\
\text { Province }\end{array}$ & Carbonate-hosted $\mathrm{Pb}-\mathrm{Zn}$ (Mississippi valley type) & $\mathrm{Pb}, \mathrm{Zn}$ & Fanhe \\
\hline K & 51 & 7 & $\begin{array}{l}\text { Xianjinchang, Qingyuan, } \\
\text { Liaoning Province }\end{array}$ & Granitoid-related $\mathrm{Au}$ vein & $\mathrm{Au}$ & Liaoji \\
\hline K & 51 & 8 & Chibaisong, Jilin Province & Mafic-ultramafic related $\mathrm{Cu}-\mathrm{Ni}$-PGE & $\mathrm{Ni}$ & Jiliaojiao \\
\hline $\mathrm{K}$ & 51 & 9 & Ermi, Jilin Province & Polymetallic $\mathrm{Pb}-\mathrm{Zn} \pm \mathrm{Cu}( \pm \mathrm{Ag}, \mathrm{Au})$ vein and stockwork & $\mathrm{Cu}$ & Jiliaolu \\
\hline K & 52 & 1 & $\begin{array}{l}\text { Huangsongdianzhi, } \\
\text { Hunchun City, Jilin } \\
\text { Province }\end{array}$ & Placer and paleoplacer $\mathrm{Au}$ & $\mathrm{Au}$ & North Jilin \\
\hline $\mathrm{K}$ & 52 & 10 & Naozhi, Jilin Province & Porphyry $\mathrm{Au}$ & $\mathrm{Au}$ & North Jilin \\
\hline K & 52 & 11 & $\begin{array}{l}\text { Tianbaoshan, Jilin } \\
\text { Province }\end{array}$ & $\mathrm{Zn}-\mathrm{Pb}(\mathrm{Ag}, \mathrm{Cu}, \mathrm{W})$ skarn & $\mathrm{Cu}, \mathrm{Pb}, \mathrm{Zn}$ & North Jilin \\
\hline $\mathrm{K}$ & 52 & 12 & Wufeng, Jilin Province & $\mathrm{Au}-\mathrm{Ag}$ epithermal vein & $\mathrm{Au}$ & North Jilin \\
\hline $\mathrm{K}$ & 52 & 13 & Changren, Jilin Province & Mafic-ultramafic related Cu-Ni-PGE & $\mathrm{Cu}$ & Hongqiling \\
\hline $\mathrm{K}$ & 52 & 14 & $\begin{array}{l}\text { Jinjia, Rongji County, } \\
\text { Jilin Province }\end{array}$ & Fluospar vein & Fluorite & North Jiling \\
\hline $\mathrm{K}$ & 52 & 15 & $\begin{array}{l}\text { Piaohechuan, Jilin } \\
\text { Province }\end{array}$ & Mafic-ultramafic related Cu-Ni-PGE & $\mathrm{Cu}$ & Hongqiling \\
\hline K & 52 & 16 & $\begin{array}{l}\text { Erdaodianzhi, Jilin } \\
\text { Province }\end{array}$ & Granitoid-related Au vein & $\mathrm{Au}$ & North Jilin \\
\hline K & 52 & 17 & $\begin{array}{l}\text { Haigou, Antu County, } \\
\text { Jilin Province }\end{array}$ & Granitoid-related $\mathrm{Au}$ vein & $\mathrm{Au}$ & North Jilin \\
\hline K & 52 & 18 & $\begin{array}{l}\text { Daheishan 2, Jilin } \\
\text { Province }\end{array}$ & Porphyry Mo $( \pm \mathrm{W}, \mathrm{Sn}, \mathrm{Bi})$ & Mo & North Jilin \\
\hline $\mathrm{K}$ & 52 & 19 & Laoniugou, Jilin Province & Banded iron formation (BIF, Algoma Fe) & $\mathrm{Fe}$ & Liaoji \\
\hline K & 52 & 2 & $\begin{array}{l}\text { Jinkuangtun, Antu } \\
\text { County, Jilin Province }\end{array}$ & $\mathrm{Au}-\mathrm{Ag}$ epithermal vein & $\mathrm{Au}$ & North Jilin \\
\hline $\mathrm{K}$ & 52 & 20 & Jiapigou, Jilin Province & $\mathrm{Au}$ in shear zone and quartz vein & $\mathrm{Au}$ & Liaoji \\
\hline $\mathrm{K}$ & 52 & 21 & Guanma, Jilin Province & Volcanic-hosted Au-base-metal metasomatite & $\mathrm{Au}$ & Hongqiling \\
\hline $\bar{K}$ & 52 & 22 & $\begin{array}{l}\text { Nanlishugou, Panshi } \\
\text { County, Jilin Province }\end{array}$ & Fluospar vein & Fluorite & North Jilin \\
\hline $\mathrm{K}$ & 52 & 23 & Sandaocha, Jilin Province & Au in shear zone and quartz vein & $\mathrm{Au}$ & Liaoji \\
\hline $\mathrm{K}$ & 52 & 24 & Hongqiling, Jilin Province & Mafic-ultramafic related $\mathrm{Cu}-\mathrm{Ni}-\mathrm{PGE}$ & $\mathrm{Cu}, \mathrm{Ni}$ & Hongqiling \\
\hline $\mathrm{K}$ & 52 & 25 & Banshigou, Jilin Province & Banded iron formation (BIF, Algoma Fe) & $\mathrm{Fe}$ & Liaoji \\
\hline K & 52 & 26 & $\begin{array}{l}\text { Liujiapuzhi (Liu } \\
\text { Daojiang), Jilin Province }\end{array}$ & Volcanic-hosted Au-base-metal metasomatite & $\mathrm{Au}$ & Jiliaolu \\
\hline K & 52 & 27 & $\begin{array}{l}\text { Erdaoyangca, Jilin } \\
\text { Province }\end{array}$ & Polymetallic $\mathrm{Pb}-\mathrm{Zn} \pm \mathrm{Cu}( \pm \mathrm{Ag}, \mathrm{Au})$ vein and stockwork & $\mathrm{Cu}$ & Jiliaolu \\
\hline $\mathrm{K}$ & 52 & 28 & Linjiang, Jilin Province & $\mathrm{Cu}( \pm \mathrm{Fe}, \mathrm{Au}, \mathrm{Ag}, \mathrm{Mo})$ skarn & $\mathrm{Cu}, \mathrm{Mo}$ & Liaoji \\
\hline $\mathrm{K}$ & 52 & 29 & Dalizi, Province & Banded iron formation (BIF, Superior Fe) & $\mathrm{Fe}$ & Jiliaojiao \\
\hline K & 52 & 3 & $\begin{array}{l}\text { Xiaoxinancha, Jilin } \\
\text { Province }\end{array}$ & Porphyry $\mathrm{Cu}( \pm \mathrm{Au})$ & $\mathrm{Cu}, \mathrm{Au}$ & North Jilin \\
\hline K & 52 & 30 & $\begin{array}{l}\text { Huanggoushan, Jilin } \\
\text { Province }\end{array}$ & Korean $\mathrm{Pb}-\mathrm{Zn}$ massive sulfide & $\mathrm{Pb}, \mathrm{Zn}$ & Jiliaojiao \\
\hline $\mathrm{K}$ & 52 & 31 & Nancha, Jilin Province & $\mathrm{Au}$ in shear zone and quartz vein & $\mathrm{Au}$ & Jiliaojiao \\
\hline K & 52 & 32 & Qidaogou, Jilin Province & Banded iron formation (BIF, Superior Fe) & $\mathrm{Fe}$ & Jiliaojiao \\
\hline $\mathrm{K}$ & 52 & 33 & Guojialing, Jilin Province & Polymetallic $(\mathrm{Pb}, \mathrm{Zn} \pm \mathrm{Cu}, \mathrm{Ba}, \mathrm{Ag}, \mathrm{Au})$ volcanic-hosted & $\mathrm{Pb}, \mathrm{Zn}$ & Jiliaolu \\
\hline
\end{tabular}




\begin{tabular}{|c|c|c|c|c|c|c|}
\hline & & & & metasomatite & & \\
\hline $\mathrm{K}$ & 52 & 4 & Tadong, Jilin Province & Volcanogenic-sedimentary Fe & $\mathrm{Fe}$ & Tadong \\
\hline $\mathrm{K}$ & 52 & 5 & $\begin{array}{l}\text { Hongtaiping, Jilin } \\
\text { Province }\end{array}$ & $\begin{array}{l}\text { Volcanogenic-hydrothermal-sedimentary massive sulfide } \\
\quad \mathrm{Pb}-\mathrm{Zn}( \pm \mathrm{Cu})\end{array}$ & $\mathrm{Pb} \mathrm{Zn}$ & Yanbian \\
\hline $\mathrm{K}$ & 52 & 6 & Ciweigou, Jilin Province & Au-Ag epithermal vein & $\mathrm{Au}, \mathrm{Ag}$ & North Jilin \\
\hline $\mathrm{K}$ & 52 & 7 & $\begin{array}{l}\text { Nongping, Hunchun City, } \\
\text { Jilin Province }\end{array}$ & Granitoid-related Au vein & $\mathrm{Au}$ & North Jilin \\
\hline K & 52 & 8 & Slavyanovskoe & Polymetallic $\mathrm{Pb}-\mathrm{Zn} \pm \mathrm{Cu}( \pm \mathrm{Ag}, \mathrm{Au})$ vein and stockwork & As & Laoeling-Grodekov \\
\hline $\bar{K}$ & 52 & 9 & $\begin{array}{l}\text { Sandaogang, Jilin } \\
\text { Province }\end{array}$ & Mafic-ultramafic related Cu-Ni-PGE & $\mathrm{Cu}$ & Hongqiling \\
\hline $\mathrm{K}$ & 53 & 1 & Fasolnoe & $\begin{array}{l}\text { Polymetallic }(\mathrm{Pb}, \mathrm{Zn} \pm \mathrm{Cu}, \mathrm{Ba}, \mathrm{Ag}, \mathrm{Au}) \text { volcanic-hosted } \\
\text { metasomatite }\end{array}$ & $\mathrm{Pb}, \mathrm{Zn}$ & Sergeevka-Taukha \\
\hline $\mathrm{K}$ & 53 & 10 & Krinichnoe & Granitoid-related $\mathrm{Au}$ vein & $\mathrm{Au}$ & Sergeevka-Taukha \\
\hline $\mathrm{K}$ & 53 & 11 & Askold & Granitoid-related $\mathrm{Au}$ vein & $\mathrm{Au}$ & Sergeevka-Taukha \\
\hline $\mathrm{K}$ & 53 & 2 & Shcherbakovskoe & Polymetallic $\mathrm{Pb}-\mathrm{Zn} \pm \mathrm{Cu}( \pm \mathrm{Ag}, \mathrm{Au})$ vein and stockwork & $\mathrm{Pb}, \mathrm{Zn}$ & Sergeevka-Taukha \\
\hline $\mathrm{K}$ & 53 & 3 & Nizhnee & Cassiterite-sulfide-silicate vein and stockwork & $\mathrm{Sn}, \mathrm{Pb}, \mathrm{Zn}$ & Luzhkinsky \\
\hline $\mathrm{K}$ & 53 & 4 & Soyuz & $\mathrm{Au}-\mathrm{Ag}$ epithermal vein & $\mathrm{Ag}, \mathrm{Au}$ & Sergeevka-Taukha \\
\hline $\mathrm{K}$ & 53 & 5 & Skalistoe & Porphyry Mo $( \pm \mathrm{W}, \mathrm{Sn}, \mathrm{Bi})$ & Mo & Samarka \\
\hline $\mathrm{K}$ & 53 & 6 & Benevskoe & $\mathrm{W} \pm \mathrm{Mo} \pm$ Be skarn & $\mathrm{W}$ & Benev \\
\hline $\mathrm{K}$ & 53 & 7 & Porozhistoe & Granitoid-related Au vein & $\mathrm{Au}$ & Sergeevka-Taukha \\
\hline $\mathrm{K}$ & 53 & 8 & Balykovskoe & Granitoid-related $\mathrm{Au}$ vein & $\mathrm{Au}$ & Sergeevka-Taukha \\
\hline $\mathrm{K}$ & 53 & 9 & Progress & Granitoid-related Au vein & $\mathrm{Au}$ & Sergeevka-Taukha \\
\hline $\mathrm{K}$ & 54 & 1 & Kitami & $\mathrm{Au}-\mathrm{Ag}$ epithermal vein & $\mathrm{Cu}, \mathrm{Pb}, \mathrm{Zn}$ & Northeast Hokkaido \\
\hline $\mathrm{K}$ & 54 & 10 & Oe & Mn vein & $\mathrm{Mn}$ & Northeast Japan \\
\hline $\mathrm{K}$ & 54 & 11 & Inakuraishi & Mn vein & $\mathrm{Mn}$ & Northeast Japan \\
\hline $\mathrm{K}$ & 54 & 12 & Toyoha & Au-Ag epithermal vein & $\mathrm{Zn}, \mathrm{Pb}, \mathrm{Ag}$ & Northeast Japan \\
\hline $\mathrm{K}$ & 54 & 13 & Todoroki & Au-Ag epithermal vein & $\mathrm{Au}, \mathrm{Ag}$ & Northeast Japan \\
\hline $\mathrm{K}$ & 54 & 14 & Kunitomi & $\begin{array}{l}\text { Volcanogenic } \mathrm{Zn}-\mathrm{Pb}-\mathrm{Cu} \text { massive sulfide (Kuroko, Altai } \\
\text { type) }\end{array}$ & $\mathrm{Zn}, \mathrm{Cu}, \mathrm{Pb}, \mathrm{Ag}$ & Northeast Japan \\
\hline $\mathrm{K}$ & 54 & 15 & Kucchan & Chemical-sedimentary Fe-Mn & $\mathrm{Fe}$ & Northeast Japan \\
\hline $\mathrm{K}$ & 54 & 16 & Chitose & Au-Ag epithermal vein & $\mathrm{Au}, \mathrm{Ag}$ & Northeast Japan \\
\hline $\mathrm{K}$ & 54 & 17 & Minamishiraoi & $\begin{array}{l}\text { Volcanogenic } \mathrm{Zn}-\mathrm{Pb}-\mathrm{Cu} \text { massive sulfide (Kuroko, Altai } \\
\text { type) }\end{array}$ & $\mathrm{Ba}$ & Northeast Japan \\
\hline $\bar{K}$ & 54 & 18 & Tokushunbetsu & Limonite from spring water & $\mathrm{Fe}$ & Northeast Japan \\
\hline $\mathrm{K}$ & 54 & 19 & Suttsu & Au-Ag epithermal vein & $\mathrm{Zn}, \mathrm{Pb}$ & Northeast Japan \\
\hline $\mathrm{K}$ & 54 & 2 & Tokoro & Clastic sediment-hosted $\mathrm{Hg} \pm \mathrm{Sb}$ & $\mathrm{Hg}$ & Northeast Hokkaido \\
\hline $\mathrm{K}$ & 54 & 20 & Horobetsu & Sulfur-sulfide $(\mathrm{S}, \mathrm{FeS} 2)$ & $\mathrm{S}$ & Northeast Japan \\
\hline $\mathrm{K}$ & 54 & 21 & Shizukari & Au-Ag epithermal vein & $\mathrm{Au}$ & Northeast Japan \\
\hline $\mathrm{K}$ & 54 & 22 & Pirika & Volcanogenic-sedimentary Mn & $\mathrm{Mn}$ & Northeast Japan \\
\hline $\mathrm{K}$ & 54 & 23 & Shojingawa & Sulfur-sulfide (S, FeS2) & $\mathrm{S}$ & Northeast Japan \\
\hline $\mathrm{K}$ & 54 & 24 & Yakumo & Mn vein & $\mathrm{Mn}, \mathrm{Zn}, \mathrm{Pb}$ & Northeast Japan \\
\hline $\mathrm{K}$ & 54 & 25 & Kinjo & Volcanogenic-sedimentary Mn & $\mathrm{Mn}$ & Northeast Japan \\
\hline $\mathrm{K}$ & 54 & 26 & Okushiri & Sulfur-sulfide (S, FeS2) & $\mathrm{S}$ & Northeast Japan \\
\hline $\mathrm{K}$ & 54 & 27 & Jokoku & Mn vein & $\mathrm{Mn}$ & Northeast Japan \\
\hline $\mathrm{K}$ & 54 & 28 & Imai-Ishizaki & Mn vein & $\mathrm{Mn}$ & Northeast Japan \\
\hline $\mathrm{K}$ & 54 & 29 & Abeshiro & $\begin{array}{l}\text { Volcanogenic } \mathrm{Zn}-\mathrm{Pb}-\mathrm{Cu} \text { massive sulfide (Kuroko, Altai } \\
\text { type) }\end{array}$ & $\mathrm{Cu}$ & Northeast Japan \\
\hline $\mathrm{K}$ & 54 & 3 & Itomuka & Volcanic-hosted $\mathrm{Hg}$ & $\mathrm{Hg}$ & Northeast Hokkaido \\
\hline K & 54 & 30 & Kamikita & $\begin{array}{l}\text { Volcanogenic } \mathrm{Zn}-\mathrm{Pb}-\mathrm{Cu} \text { massive sulfide (Kuroko, Altai } \\
\text { type) }\end{array}$ & $\mathrm{Cu}, \mathrm{Zn}$ & Northeast Japan \\
\hline $\mathrm{K}$ & 54 & 31 & Kunohe district & Volcanogenic-sedimentary Mn & $\mathrm{Mn}$ & North Kitakami \\
\hline
\end{tabular}




\begin{tabular}{|c|c|c|c|c|c|c|}
\hline Row & Col. & No. & Deposit Name & Mineral Deposit Type & Major Metals & Metallogenic Belt \\
\hline $\mathrm{K}$ & 54 & 32 & Nodatamagawa & Volcanogenic-sedimentary Mn & $\mathrm{Mn}$ & North Kitakami \\
\hline K & 54 & 33 & Funauchi & Au-Ag epithermal vein & $\mathrm{Zn}, \mathrm{Pb}, \mathrm{Cu}$ & Northeast Japan \\
\hline $\mathrm{K}$ & 54 & 34 & Furutobe & $\begin{array}{l}\text { Volcanogenic } \mathrm{Zn}-\mathrm{Pb}-\mathrm{Cu} \text { massive sulfide (Kuroko, Altai } \\
\text { type) }\end{array}$ & $\mathrm{Cu}, \mathrm{Zn}, \mathrm{Pb}, \mathrm{Ag}$ & Northeast Japan \\
\hline $\bar{K}$ & 54 & 35 & Ainai & $\begin{array}{l}\text { Volcanogenic } \mathrm{Zn}-\mathrm{Pb}-\mathrm{Cu} \text { massive sulfide (Kuroko, Altai } \\
\text { type) }\end{array}$ & $\mathrm{Zn}, \mathrm{Cu}, \mathrm{Pb}, \mathrm{Ag}$ & Northeast Japan \\
\hline $\mathrm{K}$ & 54 & 36 & Kosaka & $\begin{array}{l}\text { Volcanogenic } \mathrm{Zn}-\mathrm{Pb}-\mathrm{Cu} \text { massive sulfide (Kuroko, Altai } \\
\text { type) }\end{array}$ & $\mathrm{Zn}, \mathrm{Pb}, \mathrm{Cu}$ & Northeast Japan \\
\hline $\mathrm{K}$ & 54 & 37 & Oppu & $\mathrm{Au}-\mathrm{Ag}$ epithermal vein & $\mathrm{Zn}, \mathrm{Pb}, \mathrm{Cu}$ & Northeast Japan \\
\hline $\mathrm{K}$ & 54 & 38 & Fujikura & Volcanogenic-sedimentary Mn & $\mathrm{Mn}$ & North Kitakami \\
\hline $\bar{K}$ & 54 & 39 & Hanaoka-Fukasawa & $\begin{array}{l}\text { Volcanogenic } \mathrm{Zn}-\mathrm{Pb}-\mathrm{Cu} \text { massive sulfide (Kuroko, Altai } \\
\text { type) }\end{array}$ & $\begin{array}{l}\mathrm{Zn}, \mathrm{Pb}, \mathrm{Cu}, \mathrm{Ag}, \\
\mathrm{Au}\end{array}$ & Northeast Japan \\
\hline $\mathrm{K}$ & 54 & 4 & Nitto & Podiform chromite & $\mathrm{Cr}$ & Kamuikotan \\
\hline $\bar{K}$ & 54 & 40 & Shakanai & $\begin{array}{l}\text { Volcanogenic } \mathrm{Zn}-\mathrm{Pb}-\mathrm{Cu} \text { massive sulfide (Kuroko, Altai } \\
\text { type) }\end{array}$ & $\begin{array}{l}\mathrm{Zn}, \mathrm{Cu}, \mathrm{Pb}, \mathrm{Ag}, \\
\mathrm{Au}\end{array}$ & Northeast Japan \\
\hline K & 54 & 41 & Hanaoka & $\begin{array}{l}\text { Volcanogenic } \mathrm{Zn}-\mathrm{Pb}-\mathrm{Cu} \text { massive sulfide (Kuroko, Altai } \\
\text { type) }\end{array}$ & $\begin{array}{l}\mathrm{Zn}, \mathrm{Cu}, \mathrm{Pb}, \mathrm{Ag}, \\
\mathrm{Au}\end{array}$ & Northeast Japan \\
\hline $\mathrm{K}$ & 54 & 42 & Daira & Au-Ag epithermal vein & $\mathrm{Zn}, \mathrm{Pb}, \mathrm{Cu}$ & Northeast Japan \\
\hline K & 54 & 43 & Hanaoka-Matsumine & $\begin{array}{l}\text { Volcanogenic } \mathrm{Zn}-\mathrm{Pb}-\mathrm{Cu} \text { massive sulfide (Kuroko, Altai } \\
\text { type) }\end{array}$ & $\begin{array}{l}\mathrm{Zn}, \mathrm{Cu}, \mathrm{Pb}, \mathrm{Ag}, \\
\mathrm{Au}\end{array}$ & Northeast Japan \\
\hline $\mathrm{K}$ & 54 & 44 & Hanawa & $\begin{array}{l}\text { Volcanogenic } \mathrm{Zn}-\mathrm{Pb}-\mathrm{Cu} \text { massive sulfide (Kuroko, Altai } \\
\text { type) }\end{array}$ & $\begin{array}{l}\mathrm{Zn}, \mathrm{Cu}, \mathrm{Pb}, \mathrm{Ag}, \\
\mathrm{Au}\end{array}$ & Northeast Japan \\
\hline $\mathrm{K}$ & 54 & 45 & Hassei & $\begin{array}{l}\text { Volcanogenic } \mathrm{Zn}-\mathrm{Pb}-\mathrm{Cu} \text { massive sulfide (Kuroko, Altai } \\
\text { type) }\end{array}$ & $\mathrm{Zn}, \mathrm{Ag}$ & Northeast Japan \\
\hline $\mathrm{K}$ & 54 & 46 & Osarizawa & $\mathrm{Au}-\mathrm{Ag}$ epithermal vein & $\begin{array}{l}\mathrm{Cu}, \mathrm{Pb}, \mathrm{Zn}, \mathrm{Au}, \\
\mathrm{Ag}\end{array}$ & Northeast Japan \\
\hline $\mathrm{K}$ & 54 & 47 & Tatemata & Polymetallic $\mathrm{Pb}-\mathrm{Zn} \pm \mathrm{Cu}( \pm \mathrm{Ag}, \mathrm{Au})$ vein and stockwork & $\mathrm{Cu}, \mathrm{Zn}, \mathrm{Pb}$ & Northeast Japan \\
\hline $\mathrm{K}$ & 54 & 5 & Ganbi & Podiform chromite & $\mathrm{Cr}$ & Kamuikotan \\
\hline $\mathrm{K}$ & 54 & 6 & Hatta & Podiform chromite & $\mathrm{Cr}$ & Kamuikotan \\
\hline $\mathrm{K}$ & 54 & 7 & Teine & $\mathrm{Au}-\mathrm{Ag}$ epithermal vein & $\mathrm{Au}, \mathrm{Ag}, \mathrm{Cu}$ & Northeast Japan \\
\hline $\mathrm{K}$ & 54 & 8 & Yoichi & Au-Ag epithermal vein & $\mathrm{Zn}, \mathrm{Cu}, \mathrm{Pb}$ & Northeast Japan \\
\hline $\mathrm{K}$ & 54 & 9 & Otarumatsukura & $\begin{array}{l}\text { Volcanogenic } \mathrm{Zn}-\mathrm{Pb}-\mathrm{Cu} \text { massive sulfide (Kuroko, Altai } \\
\text { type) }\end{array}$ & $\mathrm{Ba}$ & Northeast Japan \\
\hline $\mathrm{L}$ & 45 & 1 & Sagsai & W-Mo-Be greisen, stockwork, and quartz vein & $\mathrm{W}$ & Mongol Altai \\
\hline $\mathrm{L}$ & 45 & 2 & Kelumute, Xinjiang & REE-Li pegmatite & $\mathrm{Li}, \mathrm{Be}, \mathrm{Nb}, \mathrm{Ta}$ & Altay \\
\hline $\mathrm{L}$ & 45 & 3 & Ayoubulake, Xingjiang & Muscovite pegmatite & Muscovite & Altay \\
\hline $\mathrm{L}$ & 45 & 4 & Keketuohai, Xinjiang & REE-Li pegmatite & $\mathrm{Be}, \mathrm{Ta}$ & Kelatongke \\
\hline $\mathrm{L}$ & 45 & 5 & Kalatongke, Xinjiang & Mafic-ultramafic related Cu-Ni-PGE & $\mathrm{Cu}, \mathrm{Ni}$ & Kelatongke \\
\hline $\mathrm{L}$ & 46 & 1 & Uet Ondor & Mafic-ultramafic related Ti-Fe (V) & $\mathrm{Fe}$ & Zavhanmandal-Jargalant \\
\hline $\mathrm{L}$ & 46 & 10 & Oyut tolgoi 1 & Cassiterite-sulfide-silicate vein and stockwork & $\mathrm{Sn}, \mathrm{Cu}$ & Deluun-Sagsai \\
\hline $\mathrm{L}$ & 46 & 11 & Boorch & $\mathrm{Ag}-\mathrm{Pb}$ epithermal vein & $\mathrm{Pb}, \mathrm{Ag}$ & Kurai-Tolbo Nuur \\
\hline $\mathrm{L}$ & 46 & 12 & Del Tsahir & $\mathrm{Au}$ in shear zone and quartz vein & $\mathrm{Cu}$ & Lake \\
\hline $\mathrm{L}$ & 46 & 13 & Bor uul & $\mathrm{Au}$ in shear zone and quartz vein & $\mathrm{Cu}$ & Lake \\
\hline $\mathrm{L}$ & 46 & 14 & Khardav & Sn-W greisen, stockwork, and quartz vein & $\mathrm{W}, \mathrm{Sn}$ & Altay \\
\hline $\mathrm{L}$ & 46 & 15 & Khargait 1 & W-Mo-Be greisen, stockwork, and quartz vein & $\mathrm{Be}$ & Mongolian-Altai \\
\hline $\mathrm{L}$ & 46 & 16 & Shoroot & REE-Li pegmatite & $\mathrm{Be}$ & Altay \\
\hline $\mathrm{L}$ & 46 & 17 & Angirt & REE-Li pegmatite & $\mathrm{Be}, \mathrm{Ta}, \mathrm{Nb}$ & Altai \\
\hline $\mathrm{L}$ & 46 & 18 & Bulgat & REE-Li pegmatite & $\mathrm{Be}$ & Altay \\
\hline $\mathrm{L}$ & 46 & 19 & Khurdet & REE-Li pegmatite & $\mathrm{Nb}, \mathrm{REE}$ & Altay \\
\hline $\mathrm{L}$ & 46 & 2 & Khalzan uul & Podiform chromite & $\mathrm{Cr}$ & Zavhan \\
\hline $\mathrm{L}$ & 46 & 20 & North Khuld & REE-Li pegmatite & $\mathrm{Be}$ & Altay \\
\hline
\end{tabular}




\begin{tabular}{|c|c|c|c|c|c|c|}
\hline Row & Col. & No. & Deposit Name & Mineral Deposit Type & Major Metals & Metallogenic Belt \\
\hline $\mathrm{L}$ & 46 & 21 & Bodonch & Muscovite pegmatite & Muscovite & Tseel \\
\hline $\mathrm{L}$ & 46 & 22 & Uench & REE-Li pegmatite & $\mathrm{Be}$ & Altay \\
\hline $\mathrm{L}$ & 46 & 23 & Buluket, Xinjiang & Muscovite pegmatite & Muscovite & Altay \\
\hline $\mathrm{L}$ & 46 & 24 & Alatasi, Xinjiang & Granitoid-related Au vein & $\mathrm{Au}$ & Kelatongke \\
\hline $\mathrm{L}$ & 46 & 25 & Khaltar-uul I & $\mathrm{Au}$ in shear zone and quartz vein & $\mathrm{Au}$ & Baruunkhuurai \\
\hline $\mathrm{L}$ & 46 & 26 & Khaltar Uul II & $\mathrm{Au}$ in shear zone and quartz vein & $\mathrm{Au}$ & Baruunkhuurai \\
\hline $\mathrm{L}$ & 46 & 27 & Ereen Uul & $\mathrm{Au}$ in shear zone and quartz vein & $\mathrm{Au}$ & Baruunkhuurai \\
\hline $\mathrm{L}$ & 46 & 28 & Khuld & REE-Li pegmatite & $\mathrm{Be}$ & Altay \\
\hline $\mathrm{L}$ & 46 & 3 & Olgoi Tsagaan & Polymetallic $\mathrm{Pb}-\mathrm{Zn} \pm \mathrm{Cu}( \pm \mathrm{Ag}, \mathrm{Au})$ vein and stockwork & $\mathrm{Cu}$ & Unassigned \\
\hline $\mathrm{L}$ & 46 & 4 & Alag Uul & Serpentinite-hosted asbestos & Asbestos & Lake \\
\hline $\mathrm{L}$ & 46 & 5 & Burged & $\begin{array}{l}\text { Polymetallic }(\mathrm{Pb}, \mathrm{Zn} \pm \mathrm{Cu}, \mathrm{Ba}, \mathrm{Ag}, \mathrm{Au}) \text { volcanic-hosted } \\
\text { metasomatite }\end{array}$ & $\mathrm{Cu}, \mathrm{Zn}, \mathrm{Pb}$ & Deluun-Sagsai \\
\hline $\mathrm{L}$ & 46 & 6 & Tsetserlegiin Nuruu & $\mathrm{Au}$ in shear zone and quartz vein & $\mathrm{Cu}$ & Lake \\
\hline $\mathrm{L}$ & 46 & 7 & $\begin{array}{l}\text { Aketishi, Aketishikan, } \\
\text { Xijiang }\end{array}$ & Au-Ag epithermal vein & $\mathrm{Au}$ & Hovdgol \\
\hline $\mathrm{L}$ & 46 & 8 & Jadat khar & Sn-W greisen, stockwork, and quartz vein & Sn & Mongol Altai \\
\hline $\mathrm{L}$ & 46 & 9 & Yargait & Cassiterite-sulfide-silicate vein and stockwork & $\mathrm{Sn}$ & Deluun-Sagsai \\
\hline $\mathrm{L}$ & 47 & 1 & Tomortolgoi & Banded iron formation (BIF, Superior Fe) & $\mathrm{Fe}$ & Hangai \\
\hline $\mathrm{L}$ & 47 & 10 & Saran uul & Porphyry $\mathrm{Cu}( \pm \mathrm{Au})$ & $\mathrm{Cu}$ & Central Mongolia \\
\hline $\mathrm{L}$ & 47 & 11 & Baidragiin gol & Banded iron formation (BIF, Superior Fe) & $\mathrm{Fe}$ & Baydrag \\
\hline $\mathrm{L}$ & 47 & 12 & Asgat uul & Fe skarn & $\mathrm{Fe}$ & Gentral Mongolian \\
\hline $\mathrm{L}$ & 47 & 13 & Buutsagaan & Au skarn & $\mathrm{Cu}, \mathrm{Au}, \mathrm{Fe}, \mathrm{Ag}$ & Central Mongolian \\
\hline $\mathrm{L}$ & 47 & 14 & Ondor Tsoohor uul & Volcanogenic-sedimentary Mn & $\mathrm{Mn}$ & Lake \\
\hline $\mathrm{L}$ & 47 & 15 & Bayangol 2 & Sedimentary phosphate & $\mathrm{P}$ & Tsagaanolom \\
\hline $\mathrm{L}$ & 47 & 16 & Ulaan Argalant & Fe skarn & $\mathrm{Fe}$ & Central Mongolia \\
\hline $\mathrm{L}$ & 47 & 17 & Bideriingol & Podiform chromite & $\mathrm{Cr}$ & Lake \\
\hline $\mathrm{L}$ & 47 & 18 & Gegeenii ovoo uul & Podiform chromite & $\mathrm{Cr}$ & Ikh Bogd \\
\hline $\mathrm{L}$ & 47 & 19 & Biderin gol & Sedimentary-metamorphic magnesite & Magnesite & Unassigned \\
\hline $\mathrm{L}$ & 47 & 2 & Zoogiin & Volcanogenic-sedimentary Fe & $\mathrm{Fe}$ & Hangai \\
\hline $\mathrm{L}$ & 47 & 20 & Nergui & Cyprus $\mathrm{Cu}-\mathrm{Zn}$ massive sulfide & $\mathrm{Cu}$ & Lake \\
\hline $\mathrm{L}$ & 47 & 21 & Nogoon tolgoi & Podiform chromite & $\mathrm{Cr}$ & Lake \\
\hline $\mathrm{L}$ & 47 & 22 & Ikh nart & Serpentinite-hosted asbestos & Asbestos & Unassigned \\
\hline $\mathrm{L}$ & 47 & 23 & Naran Davaa & Cyprus $\mathrm{Cu}-\mathrm{Zn}$ massive sulfide & $\mathrm{Cu}$ & Lake \\
\hline $\mathrm{L}$ & 47 & 24 & Taishir & Serpentinite-hosted asbestos & Asbestos & Lake \\
\hline $\mathrm{L}$ & 47 & 25 & Tsakhir khudag & $\mathrm{Cu}( \pm \mathrm{Fe}, \mathrm{Au}, \mathrm{Ag}, \mathrm{Mo})$ skarn & $\mathrm{Cu}-\mathrm{Au}$ & Central Mongolia \\
\hline $\mathrm{L}$ & 47 & 26 & Bayangovi district & $\mathrm{Au}$ in shear zone and quartz vein & $\mathrm{Au}$ & Bayangovi \\
\hline $\mathrm{L}$ & 47 & 27 & Tsagaan gol & Talc (magnesite) replacement & Talc & Ikh Bogd \\
\hline $\mathrm{L}$ & 47 & 28 & Sharturuutiin gol & Volcanogenic-sedimentary $\mathrm{Mn}$ & $\mathrm{Mn}$ & Govi-Altai \\
\hline $\mathrm{L}$ & 47 & 29 & Tahilgat uul & Volcanogenic-sedimentary Mn & $\mathrm{Mn}$ & Govi-Altai \\
\hline $\mathrm{L}$ & 47 & 3 & Khangai & Volcanogenic-sedimentary $\mathrm{Fe}$ & $\mathrm{Fe}$ & Hangai \\
\hline $\mathrm{L}$ & 47 & 30 & Bayantsagaan 1 & Besshi Cu-Zn-Ag massive sulfide & $\mathrm{Cu}$ & Bayanleg \\
\hline $\mathrm{L}$ & 47 & 31 & Uhiin ovoo & Volcanogenic-sedimentary Fe & $\mathrm{Fe}$ & Govi-Altai \\
\hline $\mathrm{L}$ & 47 & 32 & Bayan Undur & Bedded barite & $\mathrm{Ba}$ & Unassigned \\
\hline $\mathrm{L}$ & 47 & 33 & Olgiibulag & Volcanogenic-sedimentary Mn & $\mathrm{Mn}$ & Edrengiin \\
\hline $\mathrm{L}$ & 47 & 34 & Khadat Gunii khudag & $\mathrm{Au}$ in shear zone and quartz vein & $\mathrm{Au}$ & Edren-Zoolon \\
\hline $\mathrm{L}$ & 47 & 4 & Bogdyn Arshaan & Fe skarn & $\mathrm{Fe}$ & Central Mongolia \\
\hline $\mathrm{L}$ & 47 & 5 & Monhot & Volcanogenic-sedimentary Fe & $\mathrm{Fe}$ & Hangai \\
\hline $\mathrm{L}$ & 47 & 6 & Khokhbulgiin khondii & $\mathrm{Cu}( \pm \mathrm{Fe}, \mathrm{Au}, \mathrm{Ag}, \mathrm{Mo})$ skarn & $\mathrm{Cu}, \mathrm{Au}$ & Bayanhongor \\
\hline $\mathrm{L}$ & 47 & 7 & Zuun-Arts & Sedimentary phosphate & $\mathrm{P}$ & Tsagaanolom \\
\hline $\mathrm{L}$ & 47 & 8 & Baruun-Arts & Sedimentary phosphate & $\mathrm{P}$ & Tsagaanolom \\
\hline $\mathrm{L}$ & 47 & 9 & Tsagaantsakhir Uul & Granitoid-related Au vein & $\mathrm{Au}$ & Bayanhongor \\
\hline
\end{tabular}




\begin{tabular}{|c|c|c|c|c|c|c|}
\hline Row & Col. & No. & Deposit Name & Mineral Deposit Type & Major Metals & Metallogenic Belt \\
\hline $\mathrm{L}$ & 48 & 1 & Ulaanburd & Cassiterite-sulfide-silicate vein and stockwork & $\mathrm{Sn}$ & Eastern Mongolian-Preargunskiy \\
\hline $\mathrm{L}$ & 48 & 10 & Oortsog & Mafic-ultramafic related Cu-Ni-PGE & $\mathrm{Cu}, \mathrm{Ni}$ & Bayangol \\
\hline $\mathrm{L}$ & 48 & 11 & Suul-Undur & Fluospar vein & Fluorite & East Mongolian-Priargunskiy \\
\hline $\mathrm{L}$ & 48 & 12 & Tamirgol & Volcanogenic-sedimentary Fe & $\mathrm{Fe}$ & Tamirgol-Yoroogol \\
\hline $\mathrm{L}$ & 48 & 13 & Bayan Uul 2 & Porphyry $\mathrm{Cu}-\mathrm{Mo}( \pm \mathrm{Au}, \mathrm{Ag})$ & $\mathrm{Cu}$ & Delgerhaan \\
\hline $\mathrm{L}$ & 48 & 14 & Baga Gazar & Sn-W greisen, stockwork, and quartz vein & Sn & Central Hentii \\
\hline $\mathrm{L}$ & 48 & 15 & Sharga Ovoo & Granitoid-related $\mathrm{Au}$ vein & $\mathrm{Au}$ & Battsengel-Uyanga-Erdenedalai \\
\hline $\mathrm{L}$ & 48 & 16 & Bilkh-Uul & Fluospar vein & $\mathrm{CaF} 2$ & East Mongolian-Priargunskiy \\
\hline $\mathrm{L}$ & 48 & 17 & Zulegt & Metamorphic graphite & Graphite & Tsenhermandal-Modot \\
\hline $\mathrm{L}$ & 48 & 18 & Kharmagtai 1 & Serpentinite-hosted asbestos & Asbestos & Unassigned \\
\hline $\mathrm{L}$ & 48 & 19 & Tumurtei & Fe-Zn skarn & $\mathrm{Zn}, \mathrm{Fe}, \mathrm{Mo}$ & Govi-Ugtaal-Baruun-Urt \\
\hline $\mathrm{L}$ & 48 & 2 & $\begin{array}{l}\text { Janchivlan (Buural } \\
\text { khangai and Urt Gozgor) }\end{array}$ & Ta-Nb-REE alkaline metasomatite & $\mathrm{Ta}$ & Central Hentii \\
\hline $\mathrm{L}$ & 48 & 20 & Modon-Us & Evaporate sedimentary gypsum & Gypsum & Govi-Tamsag \\
\hline $\mathrm{L}$ & 48 & 21 & Khongoot & Porphyry $\mathrm{Cu}( \pm \mathrm{Au})$ & $\mathrm{Cu}$ & Harmagtai-Hongoot-Oyut \\
\hline $\mathrm{L}$ & 48 & 22 & Taragt & Evaporate sedimentary gypsum & Gypsum & Govi-Tamsag \\
\hline $\mathrm{L}$ & 48 & 23 & Shiree Uul (Taragt-2) & Evaporate sedimentary gypsum & Gypsum & Govi-Tamsag \\
\hline $\mathrm{L}$ & 48 & 24 & Dugshih hudag & Sedimentary celestite & $\mathrm{Sr}$ & Govi-Tamsag \\
\hline $\mathrm{L}$ & 48 & 25 & Kharmagtai 2 & Porphyry Cu-Mo $( \pm \mathrm{Au}, \mathrm{Ag})$ & $\mathrm{Cu}$ & Harmagtai-Hongoot-Oyut \\
\hline $\mathrm{L}$ & 48 & 26 & Dorvon Dert & Be tuff & $\mathrm{Be}$ & Mushgaihudag-Olgiihiid \\
\hline $\mathrm{L}$ & 48 & 27 & Olon Ovoot & $\mathrm{Au}$ in shear zone and quartz vein & $\mathrm{Au}$ & Ulziit \\
\hline $\mathrm{L}$ & 48 & 28 & Bayan Khoshuu & Barite vein & $\mathrm{Ba}$ & Unassigned \\
\hline $\mathrm{L}$ & 48 & 29 & Mushgai hudag & $\mathrm{REE}( \pm \mathrm{Ta}, \mathrm{Nb}, \mathrm{Fe})$ carbonatite & REE & Mushgaihudag-Olgiihiid \\
\hline $\mathrm{L}$ & 48 & 3 & Bayan Ovoo & Sn-W greisen, stockwork, and quartz vein & Sn, W & Central Hentii \\
\hline $\mathrm{L}$ & 48 & 30 & Khorimt khudag & Au in shear zone and quartz vein & $\mathrm{Au}$ & Ulziit \\
\hline $\mathrm{L}$ & 48 & 31 & Teg uul & Be tuff & $\mathrm{Be}$ & Mushgaihudag-Olgiihiid \\
\hline $\mathrm{L}$ & 48 & 32 & Khotgor & $\mathrm{REE}( \pm \mathrm{Ta}, \mathrm{Nb}, \mathrm{Fe})$ carbonatite & REE & Mushgaihudag-Olgiihiid \\
\hline $\mathrm{L}$ & 48 & 4 & Avdrant & Peralkaline granitoid-related Nb-Zr-REE & $\mathrm{Ta}$ & Central Hentii \\
\hline $\mathrm{L}$ & 48 & 5 & Chuluut tsagaan del & Fluospar vein & $\mathrm{CaF} 2$ & East Mongolian-Priargunskiy \\
\hline $\mathrm{L}$ & 48 & 6 & Urt Gozgor & Ta-Nb-REE alkaline metasomatite & $\mathrm{Li}, \mathrm{Ta}$, & Central Hentii \\
\hline $\mathrm{L}$ & 48 & 7 & Ikh Khairkhan & W-Mo-Be greisen, stockwork, and quartz vein & W, Mo & Ikh-Hairhan \\
\hline $\mathrm{L}$ & 48 & 8 & Ongon Khairhan & W-Mo-Be greisen, stockwork, and quartz vein & W, Mo & Ikh Hairhan \\
\hline $\mathrm{L}$ & 48 & 9 & Ongon Khairkhan & Ta-Li ongonite & $\mathrm{Ta}, \mathrm{Li}$ & Ikh Hairhan \\
\hline$\overline{\mathrm{L}}$ & 49 & 1 & Aryn nuur & Porphyry Mo $( \pm \mathrm{W}, \mathrm{Sn}, \mathrm{Bi})$ & Mo & Govi-Ugtaal-Baruun-Urt \\
\hline $\mathrm{L}$ & 49 & 10 & Tumurtiin-Ovoo & Fe-Zn skarn & $\mathrm{Zn}, \mathrm{Fe}$ & Govi-Ugtaal-Baruun-Urt \\
\hline $\mathrm{L}$ & 49 & 11 & Mongon Ondor & Polymetallic $\mathrm{Pb}-\mathrm{Zn} \pm \mathrm{Cu}( \pm \mathrm{Ag}, \mathrm{Au})$ vein and stockwork & $\mathrm{Ag}$ & East Mongolian-Priargunskiy \\
\hline $\mathrm{L}$ & 49 & 12 & Salaa & W-Mo-Be greisen, stockwork, and quartz vein & $\mathrm{W}$ & Central Mongolia \\
\hline $\mathrm{L}$ & 49 & 13 & Tsagaan chuluut & Porphyry Mo $( \pm \mathrm{W}, \mathrm{Sn}, \mathrm{Bi})$ & Mo & Eastern Mongolian-Preargunskiy \\
\hline $\mathrm{L}$ & 49 & 14 & Zaan shiree & Cassiterite-sulfide-silicate vein and stockwork & $\mathrm{Sn}$ & Eastern Mongolian-Preargunskiy \\
\hline $\mathrm{L}$ & 49 & 15 & Bees & $\mathrm{W} \pm \mathrm{Mo} \pm \mathrm{Be}$ skarn & $\mathrm{W}$ & Central Hentii \\
\hline $\mathrm{L}$ & 49 & 16 & Modot & Sn-W greisen, stockwork, and quartz vein & Sn, W & Central Hentii \\
\hline $\mathrm{L}$ & 49 & 17 & Khujkhan & Sn-W greisen, stockwork, and quartz vein & Sn, W & Central Hentii \\
\hline $\mathrm{L}$ & 49 & 18 & Bayan Mod & Sn-W greisen, stockwork, and quartz vein & Sn, W & Central Hentii \\
\hline $\mathrm{L}$ & 49 & 19 & Baruuntsogt & Ta-Nb-REE alkaline metasomatite & $\mathrm{Ta}$ & East Mongolian-Priargunskiy \\
\hline $\mathrm{L}$ & 49 & 2 & Tumen Tsogt & $\mathrm{W} \pm \mathrm{Mo} \pm \mathrm{Be}$ skarn & $\mathrm{W}$ & Eastern Mongolian-Preargunskiy \\
\hline$\overline{\mathrm{L}}$ & 49 & 20 & Tugalgatain nuruu & Sn-W greisen, stockwork, and quartz vein & $\mathrm{Zn}, \mathrm{Pb}, \mathrm{Sn}, \mathrm{Ag}$ & East Mongolian-Priargunskiy \\
\hline $\mathrm{L}$ & 49 & 21 & Galshar & Fluospar vein & $\mathrm{CaF} 2$ & East Mongolian-Priargunskiy \\
\hline $\mathrm{L}$ & 49 & 22 & Itgel Naidvar & Metamorphic graphite & Graphite & Tsenhermandal-Modot \\
\hline $\mathrm{L}$ & 49 & 23 & Khajuu Ulaan & Fluospar vein & $\mathrm{CaF} 2$ & East Mongolian-Priargunskiy \\
\hline $\mathrm{L}$ & 49 & 24 & Nars & Sediment-hosted U & $\mathrm{U}$ & Govi-Tamsag \\
\hline
\end{tabular}




\begin{tabular}{|c|c|c|c|c|c|c|}
\hline Row & Col. & No. & Deposit Name & Mineral Deposit Type & Major Metals & Metallogenic Belt \\
\hline $\mathrm{L}$ & 49 & 25 & Bor-Undur & Fluorspar vein & $\mathrm{CaF} 2$ & East Mongolian-Priargunskiy \\
\hline $\mathrm{L}$ & 49 & 26 & Khokh Del Uul & Ta-Nb-REE alkaline metasomatite & $\mathrm{Ta}, \mathrm{Nb}$ & Eastern Mongolian-Preargunskiy \\
\hline $\mathrm{L}$ & 49 & 27 & Khar Airag & Fluospar vein & $\mathrm{CaF} 2$ & East Mongolian-Priargunskiy \\
\hline $\mathrm{L}$ & 49 & 28 & Ikh Nartyn Khiid & Fluospar vein & $\mathrm{CaF} 2$ & East Mongolian-Priargunskiy \\
\hline $\mathrm{L}$ & 49 & 29 & Bujgar & Fluospar vein & $\mathrm{CaF} 2$ & East Mongolian-Priargunskiy \\
\hline $\mathrm{L}$ & 49 & 3 & Tumentsogt & W-Mo-Be greisen, stockwork, and quartz vein & $\mathrm{W}$ & Eastern Mongolian-Preargunskiy \\
\hline $\mathrm{L}$ & 49 & 30 & Ikh Zelend & W-Mo-Be greisen, stockwork, and quartz vein & $\mathrm{W}$ & Central Hentii \\
\hline $\mathrm{L}$ & 49 & 31 & Haraat & Sediment-hosted U & $\mathrm{U}$ & Govi-Tamsag \\
\hline $\mathrm{L}$ & 49 & 32 & Urgen 1 & Volcanic-hosted zeolite & Zeolite & Govi-Tamsag \\
\hline $\mathrm{L}$ & 49 & 33 & Urgen 2 & Carbonate-hosted fluorspar & Fluorite & Unassigned \\
\hline $\mathrm{L}$ & 49 & 34 & Oortsog ovoo & Sn skarn & $\mathrm{Sn}, \mathrm{Pb}, \mathrm{Zn}$ & Govi-Ugtaal-Baruun-Urt \\
\hline $\mathrm{L}$ & 49 & 35 & Tushleg & Volcanic-hosted zeolite & Zeolite & Govi-Tamsag \\
\hline $\mathrm{L}$ & 49 & 36 & Tsagaantsav & Volcanic-hosted zeolite & Zeolite & Govi-Tamsag \\
\hline $\mathrm{L}$ & 49 & 37 & Shine & Granitoid-related Au vein & $\mathrm{Au}-\mathrm{Ag}-\mathrm{Cu}$ & Harmagtai-Hongoot-Oyut \\
\hline $\mathrm{L}$ & 49 & 38 & Oyuut Ulaan ovoo & Porphyry $\mathrm{Cu}( \pm \mathrm{Au})$ & $\mathrm{Cu}$ & Harmagtai-Hongoot-Oyut \\
\hline $\mathrm{L}$ & 49 & 39 & Unegt Uul & Evaporate sedimentary gypsum & Gypsum & Govi-Tamsag \\
\hline $\mathrm{L}$ & 49 & 4 & Anas & Fluospar vein & Fluorite & East Mongolian-Priargunskiy \\
\hline $\mathrm{L}$ & 49 & 40 & Nariin khudag & Porphyry $\mathrm{Cu}( \pm \mathrm{Au})$ & $\mathrm{Cu}$ & Harmagtai-Hongoot-Oyut \\
\hline $\mathrm{L}$ & 49 & 5 & Berkh 1 & Fluospar vein & Fluorite & East Mongolian-Priargunskiy \\
\hline $\mathrm{L}$ & 49 & 6 & Khol khudag & Fe-Zn skarn & $\mathrm{Zn}, \mathrm{Fe}, \mathrm{Mo}$ & Govi-Ugtaal-Baruun-Urt \\
\hline $\mathrm{L}$ & 49 & 7 & Ondortsagan & W-Mo-Be greisen, stockwork, and quartz vein & W, Mo & Eastern Mongolian-Preargunskiy \\
\hline $\mathrm{L}$ & 49 & 8 & Omnodelger & Sn-W greisen, stockwork, and quartz vein & Sn & Central Khentii \\
\hline $\mathrm{L}$ & 49 & 9 & Mungon-Ondur & Sn-W greisen, stockwork, and quartz vein & $\mathrm{Zn}, \mathrm{Pb}, \mathrm{Sn}, \mathrm{Ag}$ & East Mongolian-Priargunskiy \\
\hline $\mathrm{L}$ & 50 & 1 & Caobulen, Inner Mongolia & $\mathrm{Zn}-\mathrm{Pb}(\mathrm{Ag}, \mathrm{Cu}, \mathrm{W})$ skarn & $\mathrm{Pb} \mathrm{Zn}$ & Daxinganling \\
\hline $\mathrm{L}$ & 50 & 2 & Modon & Polymetallic $\mathrm{Pb}-\mathrm{Zn} \pm \mathrm{Cu}( \pm \mathrm{Ag}, \mathrm{Au})$ vein and stockwork & $\mathrm{Pb}, \mathrm{Ag}$ & Nuhetdavaa \\
\hline $\mathrm{L}$ & 50 & 3 & $\begin{array}{l}\text { Aonaodaba,Inner } \\
\text { Mongolia }\end{array}$ & Polymetallic $\mathrm{Pb}-\mathrm{Zn} \pm \mathrm{Cu}( \pm \mathrm{Ag}, \mathrm{Au})$ vein and stockwork & $\mathrm{Ag}, \mathrm{Sn}$ & Daxinganling \\
\hline $\mathrm{L}$ & 50 & 4 & Haobugao,Inner Mongolia & Sn skarn & $\mathrm{Sn}$ & Daxinganling \\
\hline $\mathrm{L}$ & 50 & 5 & Yugzer & W-Mo-Be greisen, stockwork, and quartz vein & $\mathrm{W}, \mathrm{Mo}$ & Nuhetdavaa \\
\hline $\mathrm{L}$ & 50 & 6 & $\begin{array}{l}\text { Baiyinnuoer, Inner } \\
\text { Mongolia }\end{array}$ & $\mathrm{Zn}-\mathrm{Pb}(\mathrm{Ag}, \mathrm{Cu}, \mathrm{W})$ skarn & $\mathrm{Pb}, \mathrm{Zn}$ & Daxinganling \\
\hline $\mathrm{L}$ & 50 & 7 & $\begin{array}{l}\text { Hegenshan 3756, Inner } \\
\text { Mongolia }\end{array}$ & Podiform chromite & $\mathrm{Cr}$ & Hegenshan \\
\hline $\mathrm{L}$ & 50 & 8 & $\begin{array}{l}\text { Hegenshan 620, Inner } \\
\text { Mongolia }\end{array}$ & Podiform chromite & $\mathrm{Cr}$ & Hegenshan \\
\hline $\mathrm{L}$ & 50 & 9 & Maodeng, Inner Mongolia & Cassiterite-sulfide-silicate vein and stockwork & $\mathrm{Cu}$ & Daxinganling \\
\hline $\mathrm{L}$ & 51 & 1 & $\begin{array}{l}\text { Lianhuashan,Inner } \\
\text { Mongolia }\end{array}$ & Polymetallic $\mathrm{Pb}-\mathrm{Zn} \pm \mathrm{Cu}( \pm \mathrm{Ag}, \mathrm{Au})$ vein and stockwork & $\mathrm{Cu}, \mathrm{Ag}$ & Daxinganling \\
\hline $\mathrm{L}$ & 51 & 2 & $\begin{array}{l}\text { Meng'entaolegai, Inner } \\
\text { Mongolia }\end{array}$ & Polymetallic $\mathrm{Pb}-\mathrm{Zn} \pm \mathrm{Cu}( \pm \mathrm{Ag}, \mathrm{Au})$ vein and stockwork & $\mathrm{Ag}, \mathrm{Pb}, \mathrm{Zn}$ & Daxinganling \\
\hline $\mathrm{L}$ & 51 & 3 & Baerzhe, Inner Mongolia & Peralkaline granitoid-related Nb-Zr-REE & $\mathrm{Nb}, \mathrm{Ta}, \mathrm{Be}$ & Daxinganling \\
\hline $\mathrm{L}$ & 52 & 1 & $\begin{array}{l}\text { Yunshan, Luobei County, } \\
\text { Heilongjiang Province }\end{array}$ & Metamorphic graphite & Graphite & Jixi \\
\hline $\mathrm{L}$ & 52 & 10 & $\begin{array}{l}\text { Guangyi, Muling, } \\
\text { HeilongJiang Province }\end{array}$ & Magmatic graphite & Graphite & Jixi \\
\hline $\mathrm{L}$ & 52 & 11 & $\begin{array}{l}\text { Liumao, Heilongjiang } \\
\text { Province }\end{array}$ & Metamorphic graphite & Graphite & Jixi \\
\hline $\mathrm{L}$ & 52 & 12 & $\begin{array}{l}\text { Sandaogou, Heilongjiang } \\
\text { Province }\end{array}$ & Metamorphic sillimanite & Sillimanite & Jixi \\
\hline $\mathrm{L}$ & 52 & 13 & $\begin{array}{l}\text { Komissarovskoe } \\
\text { (Vorob'eva plad) }\end{array}$ & Au-Ag epithermal vein & $\mathrm{Au}, \mathrm{Ag}$ & Laoeling-Grodekov \\
\hline
\end{tabular}




\begin{tabular}{|c|c|c|c|c|c|c|}
\hline Row & Col. & No. & Deposit Name & Mineral Deposit Type & Major Metals & Metallogenic Belt \\
\hline $\mathrm{L}$ & 52 & 14 & $\begin{array}{l}\text { Mingli, Heilongjiang } \\
\text { Province }\end{array}$ & $\mathrm{Zn}-\mathrm{Pb}(\mathrm{Ag}, \mathrm{Cu}, \mathrm{W})$ skarn & $\mathrm{Zn}$ & Bindong \\
\hline $\mathrm{L}$ & 52 & 15 & $\begin{array}{l}\text { Zolotoi Stream (Sofie- } \\
\text { Alekseevskoe) }\end{array}$ & Au in shear zone and quartz vein & $\mathrm{Au}$ & Laoeling-Grodekov \\
\hline $\mathrm{L}$ & 52 & 16 & Baikal & Porphyry Cu-Mo $( \pm \mathrm{Au}, \mathrm{Ag})$ & $\mathrm{Cu}, \mathrm{Mo}$ & Laoeling-Grodekov \\
\hline $\mathrm{L}$ & 52 & 17 & $\begin{array}{l}\text { Wudaoling, Heilongjiang } \\
\text { Province }\end{array}$ & $\mathrm{W} \pm \mathrm{Mo} \pm \mathrm{Be}$ skarn & Mo & Bindong \\
\hline $\mathrm{L}$ & 52 & 18 & $\begin{array}{l}\text { Niutoushan, Jiutai } \\
\text { County, Jilin Province }\end{array}$ & Fluospar vein & Fluorite & North Jilin \\
\hline $\mathrm{L}$ & 52 & 19 & $\begin{array}{l}\text { Gongpengzi, Heilongjiang } \\
\text { Province }\end{array}$ & $\mathrm{Cu}( \pm \mathrm{Fe}, \mathrm{Au}, \mathrm{Ag}, \mathrm{Mo})$ skarn & $\mathrm{Cu}$ & Bindong \\
\hline $\mathrm{L}$ & 52 & 2 & $\begin{array}{l}\text { Dongfengshan, } \\
\text { Heilongjiang Province }\end{array}$ & Homestake $\mathrm{Au}$ & $\mathrm{Au}$ & Jixi \\
\hline $\mathrm{L}$ & 52 & 3 & $\begin{array}{l}\text { Daxilin, Heilongjiang } \\
\text { Province }\end{array}$ & $\begin{array}{l}\text { Volcanogenic-hydrothermal-sedimentary massive sulfide } \\
\mathrm{Pb}-\mathrm{Zn}( \pm \mathrm{Cu})\end{array}$ & $\mathrm{Fe}$ & Xilin \\
\hline $\mathrm{L}$ & 52 & 4 & $\begin{array}{l}\text { Shuangyashan, } \\
\text { Heilongjiang Province }\end{array}$ & Banded iron formation (BIF, Algoma Fe) & $\mathrm{Fe}$ & Jixi \\
\hline $\mathrm{L}$ & 52 & 5 & $\begin{array}{l}\text { Xiaoxilin, Heilongiiang } \\
\text { Province }\end{array}$ & $\begin{array}{l}\text { Volcanogenic-hydrothermal-sedimentary massive sulfide } \\
\qquad \mathrm{Pb}-\mathrm{Zn}( \pm \mathrm{Cu})\end{array}$ & $\mathrm{Pb}, \mathrm{Zn}$ & Xilin \\
\hline $\mathrm{L}$ & 52 & 6 & $\begin{array}{l}\text { Yangbishan, Heilongiang } \\
\text { Province }\end{array}$ & Magmatic graphite & Graphite & Jixi \\
\hline $\mathrm{L}$ & 52 & 7 & $\begin{array}{l}\text { Laozhuoshan, } \\
\text { Heilongjiang Province }\end{array}$ & Granitoid-related $\mathrm{Au}$ vein & $\mathrm{Au}$ & Laozhuoshan \\
\hline $\mathrm{L}$ & 52 & 8 & $\begin{array}{l}\text { Ergu-Xishan, Heilongjian } \\
\text { Province }\end{array}$ & $\mathrm{Zn}-\mathrm{Pb}(\mathrm{Ag}, \mathrm{Cu}, \mathrm{W})$ skarn & $\mathrm{Ag}, \mathrm{Pb}, \mathrm{Zn}$ & Bindong \\
\hline $\mathrm{L}$ & 52 & 9 & $\begin{array}{l}\text { Donghai, Jixi City, } \\
\text { Heilongjiang Province }\end{array}$ & Metamorphic graphite & Graphite & Jixi \\
\hline $\mathrm{L}$ & 53 & 1 & Khvoshchovoe & Porphyry $\mathrm{Cu}-\mathrm{Mo}( \pm \mathrm{Au}, \mathrm{Ag})$ & $\mathrm{Cu}, \mathrm{Mo}$ & Samarka \\
\hline $\mathrm{L}$ & 53 & 10 & Lermontovsky & $\mathrm{W} \pm \mathrm{Mo} \pm \mathrm{Be}$ skarn & $\mathrm{W}$ & Samarka \\
\hline $\mathrm{L}$ & 53 & 11 & Vostok-2 & $\mathrm{W} \pm \mathrm{Mo} \pm \mathrm{Be}$ skarn & $\mathrm{W}$ & Samarka \\
\hline $\mathrm{L}$ & 53 & 12 & Zvezdnoe & Porphyry Sn & Sn & Luzhkinsky \\
\hline $\mathrm{L}$ & 53 & 13 & Tigrinoe & Sn-W greisen, stockwork, and quartz vein & $\begin{array}{l}\text { Sn, W, Ta, Nb, } \\
\text { In }\end{array}$ & Luzhkinsky \\
\hline $\mathrm{L}$ & 53 & 14 & Zimnee & Sn-W greisen, stockwork, and quartz vein & $\mathrm{Sn}, \mathrm{Pb}, \mathrm{Zn}$ & Luzhkinsky \\
\hline $\mathrm{L}$ & 53 & 15 & Tayozhnoe 1 & $\mathrm{Au}-\mathrm{Ag}$ epithermal vein & $\mathrm{Ag}$ & Kema \\
\hline $\mathrm{L}$ & 53 & 16 & Dalnetayozhnoe & Sn-W greisen, stockwork, and quartz vein & $\mathrm{Sn}, \mathrm{Pb}, \mathrm{Zn}$ & Luzhkinsky \\
\hline $\mathrm{L}$ & 53 & 17 & Zabytoe & W-Mo-Be greisen, stockwork, and quartz vein & $\mathrm{W}, \mathrm{Sn}, \mathrm{Bi}$ & Luzhkinsky \\
\hline $\mathrm{L}$ & 53 & 18 & Malinovskoe & Porphyry $\mathrm{Cu}( \pm \mathrm{Au})$ & $\mathrm{Cu}$ & Luzhkinsky \\
\hline $\mathrm{L}$ & 53 & 19 & Plastun & Porphyry $\mathrm{Cu}( \pm \mathrm{Au})$ & $\mathrm{Cu}$ & Sergeevka-Taukha \\
\hline $\mathrm{L}$ & 53 & 2 & Kafen & Porphyry $\mathrm{Cu}-\mathrm{Mo}( \pm \mathrm{Au}, \mathrm{Ag})$ & $\mathrm{Cu}, \mathrm{Mo}$ & Samarka \\
\hline $\mathrm{L}$ & 53 & 20 & Ariadnoe & Mafic-ultramafic related Ti-Fe (V) & $\mathrm{Ti}$ & Ariadny \\
\hline $\mathrm{L}$ & 53 & 21 & Skrytoe & $\mathrm{W} \pm \mathrm{Mo} \pm \mathrm{Be}$ skarn & $\mathrm{W}$ & Samarka \\
\hline $\mathrm{L}$ & 53 & 22 & Yuzhnoe & Polymetallic $\mathrm{Pb}-\mathrm{Zn} \pm \mathrm{Cu}( \pm \mathrm{Ag}, \mathrm{Au})$ vein and stockwork & $\mathrm{Pb}, \mathrm{Zn}, \mathrm{Ag}$ & Luzhkinsky \\
\hline $\mathrm{L}$ & 53 & 23 & Ussuri & Banded iron formation (BIF, Superior Fe) & $\mathrm{Fe}$ & Kabarga \\
\hline $\mathrm{L}$ & 53 & 24 & Nikolaevskoe & $\mathrm{Zn}-\mathrm{Pb}(\mathrm{Ag}, \mathrm{Cu}, \mathrm{W})$ skarn & $\mathrm{Pb}, \mathrm{Zn}$ & Sergeevka-Taukha \\
\hline $\mathrm{L}$ & 53 & 25 & Smirnovskoe & Polymetallic $\mathrm{Pb}-\mathrm{Zn} \pm \mathrm{Cu}( \pm \mathrm{Ag}, \mathrm{Au})$ vein and stockwork & $\mathrm{Pb}, \mathrm{Zn}, \mathrm{Sn}$ & Luzhkinsky \\
\hline $\mathrm{L}$ & 53 & 26 & Krasnogorskoye 2 & $\begin{array}{l}\text { Polymetallic }(\mathrm{Pb}, \mathrm{Zn} \pm \mathrm{Cu}, \mathrm{Ba}, \mathrm{Ag}, \mathrm{Au}) \text { volcanic-hosted } \\
\text { metasomatite }\end{array}$ & $\mathrm{Pb}, \mathrm{Zn}$ & Sergeevka-Taukha \\
\hline $\mathrm{L}$ & 53 & 27 & Lidovskoe & Polymetallic $\mathrm{Pb}-\mathrm{Zn} \pm \mathrm{Cu}( \pm \mathrm{Ag}, \mathrm{Au})$ vein and stockwork & $\mathrm{Pb}, \mathrm{Zn}$ & Sergeevka-Taukha \\
\hline $\mathrm{L}$ & 53 & 28 & Dalnegorsk & Boron (datolite) skarn & $\mathrm{B}$ & Sergeevka-Taukha \\
\hline $\mathrm{L}$ & 53 & 29 & Partizanskoe (Soviet 2, & $\mathrm{Zn}-\mathrm{Pb}(\mathrm{Ag}, \mathrm{Cu}, \mathrm{W})$ skarn & $\mathrm{Pb}, \mathrm{Zn}$ & Sergeevka-Taukha \\
\hline
\end{tabular}


\begin{tabular}{|l|l|l|l|l|l|l|}
\hline Row & Col. & No. & Deposit Name & Mineral Deposit Type & Major Metals & Metallogenic Belt \\
\hline
\end{tabular}

\begin{tabular}{|c|c|c|c|c|c|c|}
\hline & & & Svetliy Otvod) & & & \\
\hline $\mathrm{L}$ & 53 & 3 & Katenskoe & Zoned mafic-ultramafic Cr-PGE & $\mathrm{Ti}$ & Ariadny \\
\hline $\mathrm{L}$ & 53 & 30 & Zarechnoe & Porphyry $\mathrm{Cu}( \pm \mathrm{Au})$ & $\mathrm{Cu}$ & Luzhkinsky \\
\hline $\mathrm{L}$ & 53 & 31 & Khrustalnoe & Cassiterite-sulfide-silicate vein and stockwork & Sn & Luzhkinsky \\
\hline $\mathrm{L}$ & 53 & 32 & Vysokogorskoe & Cassiterite-sulfide-silicate vein and stockwork & Sn & Luzhkinsky \\
\hline $\mathrm{L}$ & 53 & 33 & Arsenyevsky & Sn-W greisen, stockwork, and quartz vein & Sn & Luzhkinsky \\
\hline $\mathrm{L}$ & 53 & 34 & Koksharovskoe & Mafic-ultramafic related Ti-Fe (V) & $\mathrm{Ti}$ & Ariadny \\
\hline $\mathrm{L}$ & 53 & 35 & Lazurnoe & Porphyry Cu-Mo $( \pm \mathrm{Au}, \mathrm{Ag})$ & $\mathrm{Cu}, \mathrm{Mo}$ & Luzhkinsky \\
\hline $\mathrm{L}$ & 53 & 36 & Chernyshevskoe & Korean $\mathrm{Pb}-\mathrm{Zn}$ massive sulfide & $\mathrm{Zn}, \mathrm{Pb}$ & Voznesenka \\
\hline $\mathrm{L}$ & 53 & 37 & Voznesenka-I & Korean $\mathrm{Pb}-\mathrm{Zn}$ massive sulfide & $\mathrm{Zn}$ & Voznesenka \\
\hline $\mathrm{L}$ & 53 & 38 & Yaroslavskoe & Sn-W greisen, stockwork, and quartz vein & Sn & Yaroslavka \\
\hline $\mathrm{L}$ & 53 & 39 & Voznesenka-II & Fluorite greisen & Fluorite & Yaroslavka \\
\hline $\mathrm{L}$ & 53 & 4 & Salyut & $\mathrm{Au}-\mathrm{Ag}$ epithermal vein & $\mathrm{Au}, \mathrm{Ag}$ & Kema \\
\hline $\mathrm{L}$ & 53 & 5 & Glinyanoe & Au-Ag epithermal vein & $\mathrm{Au}, \mathrm{Ag}$ & Kema \\
\hline $\mathrm{L}$ & 53 & 6 & Malakhitovoe & Porphyry Cu-Mo $( \pm \mathrm{Au}, \mathrm{Ag})$ & $\mathrm{Cu}, \mathrm{Mo}$ & Samarka \\
\hline $\mathrm{L}$ & 53 & 7 & Verkhnezolotoe & Porphyry $\mathrm{Cu}( \pm \mathrm{Au})$ & $\mathrm{Cu}, \mathrm{Sn}$ & Kema? \\
\hline $\mathrm{L}$ & 53 & 8 & Nesterovskoe & Porphyry $\mathrm{Cu}( \pm \mathrm{Au})$ & $\mathrm{Cu}$ & Kema \\
\hline $\mathrm{L}$ & 53 & 9 & Yantarnoe & Porphyry Sn & $\mathrm{Sn}$ & Luzhkinsky \\
\hline $\mathrm{L}$ & 54 & 1 & Yagodnoe & $\mathrm{Au}-\mathrm{Ag}$ epithermal vein & $\mathrm{Au}, \mathrm{Ag}$ & Kema \\
\hline $\mathrm{L}$ & 54 & 2 & Burmatovskoe & Au-Ag epithermal vein & $\mathrm{Au}, \mathrm{Ag}$ & Kema \\
\hline $\mathrm{L}$ & 54 & 3 & Sukhoe & Au-Ag epithermal vein & $\mathrm{Au}, \mathrm{Ag}$ & Kema \\
\hline $\mathrm{L}$ & 54 & 4 & Motokura & Au-Ag epithermal vein & $\mathrm{Pb}, \mathrm{Zn}, \mathrm{Cu}$ & Northeast Hokkaido \\
\hline $\mathrm{L}$ & 54 & 5 & Ryushoden & $\mathrm{Hg}$-Sb-W vein and stockwork & $\mathrm{Hg}$ & Northeast Hokkaido \\
\hline $\mathrm{L}$ & 54 & 6 & Numanoue & Au-Ag epithermal vein & $\mathrm{Ag}, \mathrm{Au}$ & Northeast Hokkaido \\
\hline $\mathrm{L}$ & 54 & 7 & Sanru & $\mathrm{Au}-\mathrm{Ag}$ epithermal vein & $\mathrm{Au}, \mathrm{Ag}$ & Northeast Hokkaido \\
\hline $\mathrm{L}$ & 54 & 8 & Konomai & Au-Ag epithermal vein & $\mathrm{Au}, \mathrm{Ag}$ & Northeast Hokkaido \\
\hline $\mathrm{L}$ & 54 & 9 & Shimokawa & Besshi Cu-Zn-Ag massive sulfide & $\mathrm{Cu}, \mathrm{Zn}, \mathrm{Co}$ & Hidaka \\
\hline $\mathrm{M}$ & 44 & 1 & Alexandrovskoye 1 & W-Mo-Be greisen, stockwork, and quartz vein & $\mathrm{W}$ & Kolyvansk \\
\hline $\mathrm{M}$ & 44 & 10 & Maslenskoye & $\begin{array}{l}\text { Volcanogenic } \mathrm{Zn}-\mathrm{Pb}-\mathrm{Cu} \text { massive sulfide (Kuroko, Altai } \\
\text { type) }\end{array}$ & $\mathrm{Cu}$ & Rudny Altai \\
\hline $\mathrm{M}$ & 44 & 11 & Korgon & Volcanogenic-sedimentary Fe & $\mathrm{Fe}$ & Korgon-Kholzun \\
\hline $\mathrm{M}$ & 44 & 12 & Inskoye & Fe skarn & $\mathrm{Fe}$ & Korgon-Kholzun \\
\hline $\mathrm{M}$ & 44 & 13 & Zacharovskoye & $\begin{array}{l}\text { Volcanogenic } \mathrm{Zn}-\mathrm{Pb}-\mathrm{Cu} \text { massive sulfide (Kuroko, Altai } \\
\text { type) }\end{array}$ & $\mathrm{Zn}, \mathrm{Pb}, \mathrm{Cu}$ & Rudny Altai \\
\hline $\mathrm{M}$ & 44 & 14 & Stepnoye & $\begin{array}{l}\text { Volcanogenic } \mathrm{Zn}-\mathrm{Pb}-\mathrm{Cu} \text { massive sulfide (Kuroko, Altai } \\
\text { type) }\end{array}$ & $\mathrm{Pb}, \mathrm{Zn}$ & Rudny Altai \\
\hline $\mathrm{M}$ & 44 & 15 & Talovskoye & $\begin{array}{l}\text { Volcanogenic } \mathrm{Zn}-\mathrm{Pb}-\mathrm{Cu} \text { massive sulfide (Kuroko, Altai } \\
\text { type) }\end{array}$ & $\mathrm{Zn}, \mathrm{Pb}, \mathrm{Cu}$ & Rudny Altai \\
\hline $\mathrm{M}$ & 44 & 16 & Kolivanskoye & W-Mo-Be greisen, stockwork, and quartz vein & $\mathrm{W}$ & Kolyvansk \\
\hline M & 44 & 17 & Rubtsovskoye & $\begin{array}{l}\text { Volcanogenic } \mathrm{Zn}-\mathrm{Pb}-\mathrm{Cu} \text { massive sulfide (Kuroko, Altai } \\
\text { type) }\end{array}$ & $\mathrm{Cu}, \mathrm{Zn}, \mathrm{Pb}$ & Rudny Altai \\
\hline $\mathrm{M}$ & 44 & 18 & Cherepanovskoye & $\begin{array}{l}\text { Polymetallic }(\mathrm{Pb}, \mathrm{Zn} \pm \mathrm{Cu}, \mathrm{Ba}, \mathrm{Ag}, \mathrm{Au}) \text { volcanic-hosted } \\
\text { metasomatite }\end{array}$ & $\mathrm{Pb}, \mathrm{Zn}$ & Rudny Altai \\
\hline $\mathrm{M}$ & 44 & 19 & Mayskoye 2 & $\begin{array}{l}\text { Volcanogenic } \mathrm{Zn}-\mathrm{Pb}-\mathrm{Cu} \text { massive sulfide (Kuroko, Altai } \\
\text { type) }\end{array}$ & $\mathrm{Pb}, \mathrm{Zn}$ & Rudny Altai \\
\hline $\mathrm{M}$ & 44 & 2 & Mulchichinskoye & W-Mo-Be greisen, stockwork, and quartz vein & $\mathrm{W}, \mathrm{Mo}$ & Kolyvansk \\
\hline $\mathrm{M}$ & 44 & 20 & Korbalihinskoye & $\begin{array}{l}\text { Volcanogenic } \mathrm{Zn}-\mathrm{Pb}-\mathrm{Cu} \text { massive sulfide (Kuroko, Altai } \\
\text { type) }\end{array}$ & $\mathrm{Zn}, \mathrm{Pb}, \mathrm{Cu}$ & Rudny Altai \\
\hline $\mathrm{M}$ & 44 & 21 & Tushkanikhinskoye & $\begin{array}{l}\text { Volcanogenic } \mathrm{Zn}-\mathrm{Pb}-\mathrm{Cu} \text { massive sulfide (Kuroko, Altai } \\
\text { type) }\end{array}$ & $\mathrm{Pb}, \mathrm{Zn}$ & Rundy Altai \\
\hline $\mathrm{M}$ & 44 & 22 & Lazurskoye & Volcanogenic $\mathrm{Zn}-\mathrm{Pb}-\mathrm{Cu}$ massive sulfide (Kuroko, Altai & $\mathrm{Pb}, \mathrm{Zn}$ & Rudny Altai \\
\hline
\end{tabular}




\begin{tabular}{|c|c|c|c|c|c|c|}
\hline & & & & type) & & \\
\hline $\mathrm{M}$ & 44 & 23 & Semenovskoye & $\begin{array}{l}\text { Volcanogenic } \mathrm{Zn}-\mathrm{Pb}-\mathrm{Cu} \text { massive sulfide (Kuroko, Altai } \\
\text { type) }\end{array}$ & $\mathrm{Zn}, \mathrm{Pb}, \mathrm{Cu}$ & Rudny Altai \\
\hline $\mathrm{M}$ & 44 & 24 & Srednee & $\begin{array}{l}\text { Volcanogenic } \mathrm{Zn}-\mathrm{Pb}-\mathrm{Cu} \text { massive sulfide (Kuroko, Altai } \\
\text { type) }\end{array}$ & $\mathrm{Pb}, \mathrm{Zn}$ & Rudny Altai \\
\hline $\mathrm{M}$ & 44 & 25 & Zarechenskoye & $\begin{array}{l}\text { Volcanogenic } \mathrm{Zn}-\mathrm{Pb}-\mathrm{Cu} \text { massive sulfide (Kuroko, Altai } \\
\text { type) }\end{array}$ & $\mathrm{Au}, \mathrm{Pb}, \mathrm{Zn}, \mathrm{Ag}$ & Rudny Altai \\
\hline $\mathrm{M}$ & 44 & 26 & Zmeinogorskoye & $\begin{array}{l}\text { Volcanogenic } \mathrm{Zn}-\mathrm{Pb}-\mathrm{Cu} \text { massive sulfide (Kuroko, Altai } \\
\text { type) }\end{array}$ & $\mathrm{Zn}, \mathrm{Pb}, \mathrm{Cu}, \mathrm{Au}$ & Rudny Altai \\
\hline $\mathrm{M}$ & 44 & 27 & Beloretskoye & Fe skarn & $\mathrm{Fe}$ & Korgon-Kholzun \\
\hline $\mathrm{M}$ & 44 & 28 & Beloretsskoye & $\mathrm{W} \pm \mathrm{Mo} \pm \mathrm{Be}$ skarn & $\mathrm{W}, \mathrm{Be}$ & Kolyvansk \\
\hline $\mathrm{M}$ & 44 & 29 & Loktevskoye & $\begin{array}{l}\text { Volcanogenic } \mathrm{Zn}-\mathrm{Pb}-\mathrm{Cu} \text { massive sulfide (Kuroko, Altai } \\
\text { type) }\end{array}$ & $\mathrm{Pb}, \mathrm{Zn}$ & Rudny Altai \\
\hline $\mathrm{M}$ & 44 & 3 & Kazancevskoye & W-Mo-Be greisen, stockwork, and quartz vein & $\mathrm{W}$ & Kolyvansk \\
\hline $\mathrm{M}$ & 44 & 30 & Jubileinoye & $\begin{array}{l}\text { Volcanogenic } \mathrm{Zn}-\mathrm{Pb}-\mathrm{Cu} \text { massive sulfide (Kuroko, Altai } \\
\text { type) }\end{array}$ & $\mathrm{Zn}, \mathrm{Pb}, \mathrm{Cu}$ & Rudny Altai \\
\hline $\mathrm{M}$ & 44 & 31 & Yubileinoye 2 & $\begin{array}{l}\text { Volcanogenic } \mathrm{Zn}-\mathrm{Pb}-\mathrm{Cu} \text { massive sulfide (Kuroko, Altai } \\
\text { type) }\end{array}$ & $\mathrm{Zn}, \mathrm{Pb}, \mathrm{Cu}$ & Rudny Altai \\
\hline $\mathrm{M}$ & 44 & 32 & Kruchkovskoye & $\begin{array}{l}\text { Volcanogenic } \mathrm{Zn}-\mathrm{Pb}-\mathrm{Cu} \text { massive sulfide (Kuroko, Altai } \\
\text { type) }\end{array}$ & $\mathrm{Cu}, \mathrm{Pb}, \mathrm{Zn}$ & Rudny Altai \\
\hline $\mathrm{M}$ & 44 & 33 & Zolotushinskoye & $\begin{array}{l}\text { Volcanogenic } \mathrm{Zn}-\mathrm{Pb}-\mathrm{Cu} \text { massive sulfide (Kuroko, Altai } \\
\text { type) }\end{array}$ & $\mathrm{Zn}, \mathrm{Cu}, \mathrm{Pb}$ & Rudny Altai \\
\hline $\mathrm{M}$ & 44 & 34 & Novo-Zolotushinskoye & $\begin{array}{l}\text { Volcanogenic } \mathrm{Zn}-\mathrm{Pb}-\mathrm{Cu} \text { massive sulfide (Kuroko, Altai } \\
\text { type) }\end{array}$ & $\mathrm{Zn}, \mathrm{Cu}, \mathrm{Pb}$ & Rudny Altai \\
\hline $\mathrm{M}$ & 44 & 4 & Chernukhinskoye & W-Mo-Be greisen, stockwork, and quartz vein & $\mathrm{W}$ & Kolyvansk \\
\hline $\mathrm{M}$ & 44 & 5 & Verkhne-Sludianskoye & W-Mo-Be greisen, stockwork, and quartz vein & $\mathrm{W}$ & Kolyvansk \\
\hline $\mathrm{M}$ & 44 & 6 & Chagyrskoye & Polymetallic $(\mathrm{Pb}, \mathrm{Zn}, \mathrm{Ag})$ carbonate-hosted metasomatite & $\mathrm{Pb}, \mathrm{Zn}$ & Korgon-Kholzun \\
\hline $\mathrm{M}$ & 44 & 7 & Plotbistchenskoye & W-Mo-Be greisen, stockwork, and quartz vein & Mo & Kolyvansk \\
\hline $\mathrm{M}$ & 44 & 8 & Chesnokovskoye & Fe skarn & $\mathrm{Fe}$ & Korgon-Kholzun \\
\hline $\mathrm{M}$ & 44 & 9 & Kharlovskoye & Zoned mafic-ultramafic Cr-PGE & $\mathrm{Fe}, \mathrm{Ti}$ & Korgon-Kholzun \\
\hline $\mathrm{M}$ & 45 & 1 & Karagosh & W-Mo-Be greisen, stockwork, and quartz vein & Mo & Kolyvansk \\
\hline $\mathrm{M}$ & 45 & 10 & Ustaurikhinskoye & W-Mo-Be greisen, stockwork, and quartz vein & $\mathrm{W}$ & Kolyvansk \\
\hline $\mathrm{M}$ & 45 & 11 & Munguntayga & W-Mo-Be greisen, stockwork, and quartz vein & Mo & Kalgutinsk \\
\hline $\mathrm{M}$ & 45 & 12 & Osinovskoye & W-Mo-Be greisen, stockwork, and quartz vein & $\mathrm{W}$ & Kolyvansk \\
\hline $\mathrm{M}$ & 45 & 13 & Cheremshanskoye & Carbonate-hosted $\mathrm{Hg}-\mathrm{Sb}$ & $\mathrm{Hg}$ & Sarasinsk \\
\hline $\mathrm{M}$ & 45 & 14 & Ilyinskoye & Sedimentary exhalative $\mathrm{Pb}-\mathrm{Zn}$ (SEDEX) & $\mathrm{Pb}, \mathrm{Zn}$ & Shirgaita \\
\hline $\mathrm{M}$ & 45 & 15 & Ivankinskoye & W-Mo-Be greisen, stockwork, and quartz vein & $\mathrm{W}$ & Kolyvansk \\
\hline $\mathrm{M}$ & 45 & 16 & Asgat & Ag-Sb vein & $\mathrm{Ag}, \mathrm{Sb}$ & Kurai-Tolbo Nuur \\
\hline M & 45 & 17 & Ozernoye 1 & $\begin{array}{l}\text { Volcanogenic-hydrothermal-sedimentary massive sulfide } \\
\mathrm{Pb}-\mathrm{Zn}( \pm \mathrm{Cu})\end{array}$ & $\mathrm{Ag}, \mathrm{Sb}$ & Kurai-Tolbo Nuur \\
\hline $\mathrm{M}$ & 45 & 18 & Shirgaita & Sedimentary exhalative $\mathrm{Pb}-\mathrm{Zn}$ (SEDEX) & $\mathrm{Pb}, \mathrm{Zn}$ & Shirgaita \\
\hline $\mathrm{M}$ & 45 & 19 & Krasnogorskoye 1 & Silica-carbonate (listvenite) $\mathrm{Hg}$ & $\mathrm{Hg}$ & Kuraiy \\
\hline $\mathrm{M}$ & 45 & 2 & Jaryshol & Fe skarn & $\mathrm{Fe}$ & Unassigned \\
\hline $\mathrm{M}$ & 45 & 20 & Kyzylchin & Polymetallic $\mathrm{Pb}-\mathrm{Zn} \pm \mathrm{Cu}( \pm \mathrm{Ag}, \mathrm{Au})$ vein and stockwork & $\mathrm{Zn}, \mathrm{Pb}$ & Korgon-Kholzunskiy \\
\hline $\mathrm{M}$ & 45 & 21 & Chagan-Uzunskoye & Silica-carbonate (listvenite) $\mathrm{Hg}$ & $\mathrm{Hg}$ & Kurai-Tolbo Nuur \\
\hline $\mathrm{M}$ & 45 & 22 & Kazandinskoye & W-Mo-Be greisen, stockwork, and quartz vein & $\mathrm{W}$ & Kolyvansk \\
\hline $\mathrm{M}$ & 45 & 23 & Aktashskoye & Carbonate-hosted Hg-Sb & $\mathrm{Hg}$ & Kurai-Tolbo Nuur \\
\hline $\mathrm{M}$ & 45 & 24 & Chagan-Burgazy & Polymetallic $\mathrm{Pb}-\mathrm{Zn} \pm \mathrm{Cu}( \pm \mathrm{Ag}, \mathrm{Au})$ vein and stockwork & $\mathrm{Ag}, \mathrm{Pb}$ & Kalgutinsk \\
\hline $\mathrm{M}$ & 45 & 25 & Ursulskoye & $\begin{array}{l}\text { Volcanogenic } \mathrm{Zn}-\mathrm{Pb}-\mathrm{Cu} \text { massive sulfide (Kuroko, Altai } \\
\text { type) }\end{array}$ & $\mathrm{Pb}, \mathrm{Zn}$ & Shirgaita \\
\hline $\mathrm{M}$ & 45 & 26 & Rudny Log & Volcanogenic-sedimentary $\mathrm{Fe}$ & $\mathrm{Fe}$ & Korgon-Kholzun \\
\hline
\end{tabular}




\begin{tabular}{|c|c|c|c|c|c|c|}
\hline $\mathrm{M}$ & 45 & 27 & Timofeevskoe & Fe skarn & $\mathrm{Fe}$ & Korgon-Kholzun \\
\hline $\mathrm{M}$ & 45 & 28 & Kazinikhinskoye & $\begin{array}{l}\text { Polymetallic }(\mathrm{Pb}, \mathrm{Zn} \pm \mathrm{Cu}, \mathrm{Ba}, \mathrm{Ag}, \mathrm{Au}) \text { volcanic-hosted } \\
\text { metasomatite }\end{array}$ & $\mathrm{Zn}, \mathrm{Pb}, \mathrm{Cu}$ & Korgon-Kholzunskiy \\
\hline M & 45 & 29 & Karagem & Co skarn & $\mathrm{Co}$ & Unassigned \\
\hline $\mathrm{M}$ & 45 & 3 & Sinyukhinskoye & Au skarn & $\mathrm{Au}$ & Martaiginsk \\
\hline $\mathrm{M}$ & 45 & 30 & Khuren Khairkhan uul & Bedded barite & $\mathrm{Ba}$ & Unassigned \\
\hline $\mathrm{M}$ & 45 & 31 & Krasnoyarskoye & $\begin{array}{l}\text { Polymetallic }(\mathrm{Pb}, \mathrm{Zn} \pm \mathrm{Cu}, \mathrm{Ba}, \mathrm{Ag}, \mathrm{Au}) \text { volcanic-hosted } \\
\text { metasomatite }\end{array}$ & $\mathrm{Pb}, \mathrm{Zn}$ & Korgon-Kholzunskiy \\
\hline $\mathrm{M}$ & 45 & 32 & Toshint Uul & $\begin{array}{l}\text { Volcanogenic } \mathrm{Zn}-\mathrm{Pb}-\mathrm{Cu} \text { massive sulfide (Kuroko, Altai } \\
\text { type) }\end{array}$ & $\mathrm{Cu}$ & Deluun-Sagsai \\
\hline $\mathrm{M}$ & 45 & 33 & Ulaan khus & Clastic sediment-hosted $\mathrm{Hg} \pm \mathrm{Sb}$ & $\mathrm{Hg}$ & Kurai-Tolbo Nuur \\
\hline M & 45 & 34 & Urzarsaiskoye & W-Mo-Be greisen, stockwork, and quartz vein & $\mathrm{W}$ & Kalgutinsk \\
\hline $\mathrm{M}$ & 45 & 35 & Uzuurtolgoi & $\begin{array}{l}\text { Volcanogenic } \mathrm{Zn}-\mathrm{Pb}-\mathrm{Cu} \text { massive sulfide (Kuroko, Altai } \\
\text { type) }\end{array}$ & $\mathrm{Zn}, \mathrm{Pb}$ & Uzuurtolgoi \\
\hline $\mathrm{M}$ & 45 & 36 & Vladimirovskoye & 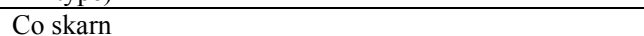 & Co & Unassigned \\
\hline $\mathrm{M}$ & 45 & 37 & Akkemskoye & W-Mo-Be greisen, stockwork, and quartz vein & Mo & Kalgutinsk \\
\hline $\mathrm{M}$ & 45 & 38 & Malachite & $\begin{array}{l}\text { Volcanogenic } \mathrm{Zn}-\mathrm{Pb}-\mathrm{Cu} \text { massive sulfide (Kuroko, Altai } \\
\text { type) }\end{array}$ & $\mathrm{Cu}$ & Uzuurtolgoi \\
\hline $\mathrm{M}$ & 45 & 39 & Kalgutinskoye 1 & W-Mo-Be greisen, stockwork, and quartz vein & $\mathrm{W}, \mathrm{Mo}$ & Kalgutinsk \\
\hline M & 45 & 4 & Kulbich & Porphyry Cu-Mo $( \pm \mathrm{Au}, \mathrm{Ag})$ & $\mathrm{Cu}, \mathrm{Mo}$ & Unassigned \\
\hline $\mathrm{M}$ & 45 & 40 & Kalgutinskoye 2 & Volcanogenic-sedimentary $\mathrm{Fe}$ & $\mathrm{Fe}$ & Korgon-Kholzun \\
\hline $\mathrm{M}$ & 45 & 41 & Mushgu & $\mathrm{Hg}-\mathrm{Sb}-\mathrm{W}$ vein and stockwork & $\mathrm{W}, \mathrm{Sb}$ & Hovdgol \\
\hline $\mathrm{M}$ & 45 & 42 & Onhot uul & Sediment-hosted $\mathrm{Cu}$ & $\mathrm{Cu}$ & Deluun-Sagsai \\
\hline $\mathrm{M}$ & 45 & 43 & Akalakhinskoye & Alkaline complex-hosted $\mathrm{Au}$ & $\begin{array}{l}\mathrm{Li}, \mathrm{Ta}, \mathrm{Nb}, \\
\text { REE }\end{array}$ & Kalgutinsk \\
\hline M & 45 & 44 & Kara-Alakha & W-Mo-Be greisen, stockwork, and quartz vein & $\mathrm{W}$ & Kalgutinsk \\
\hline M & 45 & 45 & Khovd gol & W-Mo-Be greisen, stockwork, and quartz vein & $\mathrm{W}, \mathrm{Sb}$ & Mongol Altai \\
\hline $\mathrm{M}$ & 45 & 46 & Koksinskoye & Volcanogenic-sedimentary $\mathrm{Fe}$ & $\mathrm{Fe}$ & Korgon-Kholzun \\
\hline $\mathrm{M}$ & 45 & 47 & Dungerekh & W-Mo-Be greisen, stockwork, and quartz vein & Mo, W & Mongol Altai \\
\hline M & 45 & 48 & Kok-Kolskoye & W-Mo-Be greisen, stockwork, and quartz vein & $\mathrm{W}, \mathrm{Mo}$ & Kalgutinsk \\
\hline $\mathrm{M}$ & 45 & 49 & Kholzunskoye & Volcanogenic-sedimentary $\mathrm{Fe}$ & $\mathrm{Fe}$ & Korgon-Kholzun \\
\hline $\mathrm{M}$ & 45 & 5 & Osokinskoye & W-Mo-Be greisen, stockwork, and quartz vein & $\mathrm{W}$ & Kolyvansk \\
\hline M & 45 & 50 & Nominy Am & Polymetallic $\mathrm{Pb}-\mathrm{Zn} \pm \mathrm{Cu}( \pm \mathrm{Ag}, \mathrm{Au})$ vein and stockwork & $\mathrm{Cu}$ & Deluun-Sagsai \\
\hline $\mathrm{M}$ & 45 & 51 & Chindagatuiskoye & W-Mo-Be greisen, stockwork, and quartz vein & $\mathrm{W}, \mathrm{Mo}$ & Kalgutinsk \\
\hline $\mathrm{M}$ & 45 & 52 & Qibeiling, Xinjiang & Muscovite pegmatite & Muscovite & Altay \\
\hline M & 45 & 53 & Qilinmutaer, Xinjiang & Muscovite pegmatite & Muscovite & Altay \\
\hline $\mathrm{M}$ & 45 & 6 & Baliktigkhem & Sn-W greisen, stockwork, and quartz vein & $\mathrm{Sn}$ & Kalgutinsk \\
\hline $\mathrm{M}$ & 45 & 7 & Sarasinskoye & Carbonate-hosted $\mathrm{Hg}-\mathrm{Sb}$ & $\mathrm{Hg}$ & Sarasinsk \\
\hline $\mathrm{M}$ & 45 & 8 & Sary-Gimatei & Carbonate-hosted Pb-Zn (Mississippi valley type) & $\mathrm{Pb}, \mathrm{Zn}$ & Unassigned \\
\hline $\mathrm{M}$ & 45 & 9 & Batunkovskoye & W-Mo-Be greisen, stockwork, and quartz vein & $\mathrm{W}$ & Kolyvansk \\
\hline $\mathrm{M}$ & 46 & 1 & Proezdnoye & Granitoid-related Au vein & $\mathrm{Au}$ & Ondumsk \\
\hline $\mathrm{M}$ & 46 & 10 & Karasugskoye & Fe-REE carbonatite & $\begin{array}{l}\text { REE, Fe, CaF2, } \\
\text { BaSO4 }\end{array}$ & Karasug \\
\hline $\mathrm{M}$ & 46 & 11 & Mugurskoye & Banded iron formation (BIF, Superior Fe) & $\mathrm{Fe}$ & Mugursk \\
\hline $\mathrm{M}$ & 46 & 12 & Eligkhem & Granitoid-related $\mathrm{Au}$ vein & $\mathrm{Au}$ & Unassigned \\
\hline $\mathrm{M}$ & 46 & 13 & Ulatayskoye & Fe-REE carbonatite & Fe, REE & Karasug \\
\hline $\mathrm{M}$ & 46 & 14 & Shuden uul & Evaporate halite & Halite & Unassigned \\
\hline $\mathrm{M}$ & 46 & 15 & Davst uul & Evaporate halite & Halite & Unassigned \\
\hline M & 46 & 16 & Chergak & $\mathrm{Ni}-\mathrm{Co}$ arsenide vein & $\mathrm{Co}, \mathrm{Cu}, \mathrm{Ni}$ & Chergak \\
\hline $\mathrm{M}$ & 46 & 17 & Actovrak & Serpentinite-hosted asbestos & Chrysotile & Khemchik-Kurtushubinsk \\
\hline $\mathrm{M}$ & 46 & 18 & Oyut tolgoi 2 & Mafic-ultramafic related $\mathrm{Cu}-\mathrm{Ni}$-PGE & $\mathrm{Cu}$ & Telmen \\
\hline
\end{tabular}




\begin{tabular}{|c|c|c|c|c|c|c|}
\hline $\mathrm{M}$ & 46 & 19 & Pertoyskoye & $\begin{array}{l}\text { Polymetallic }(\mathrm{Pb}, \mathrm{Zn} \pm \mathrm{Cu}, \mathrm{Ba}, \mathrm{Ag}, \mathrm{Au}) \text { volcanic-hosted } \\
\text { metasomatite }\end{array}$ & $\mathrm{Pb}, \mathrm{Zn}$ & Unassigned \\
\hline $\mathrm{M}$ & 46 & 2 & Tardan & Au skarn & $\mathrm{Au}$ & Ondumsk \\
\hline M & 46 & 20 & Tsagdaltyn Davaa & Mafic-ultramafic related $\mathrm{Cu}-\mathrm{Ni}$-PGE & $\mathrm{Ni}$ & Lake \\
\hline $\mathrm{M}$ & 46 & 21 & Tomorchuluut & Banded iron formation (BIF, Superior Fe) & $\mathrm{Fe}$ & Khan Hokhii \\
\hline $\mathrm{M}$ & 46 & 22 & Tolailyk & $\mathrm{Ni}-\mathrm{Co}$ arsenide vein & $\mathrm{Co}, \mathrm{Cu}$ & Chergak \\
\hline $\mathrm{M}$ & 46 & 23 & Borts Uul & Volcanogenic $\mathrm{Cu}-\mathrm{Zn}$ massive sulfide (Urals type) & $\mathrm{Cu}$ & Lake \\
\hline $\mathrm{M}$ & 46 & 24 & Chazadyrskoye & Silica-carbonate (listvenite) $\mathrm{Hg}$ & $\mathrm{Hg}$ & Terligkhaisk \\
\hline $\mathrm{M}$ & 46 & 25 & Ulaantolgoi & Peralkaline granitoid-related Nb-Zr-REE & $\begin{array}{l}\mathrm{Zr}, \mathrm{Nb}, \mathrm{Ta}, \\
\mathrm{REE}\end{array}$ & \\
\hline & Khalzanburged & & & & & \\
\hline $\mathrm{M}$ & 46 & 26 & Bayankhairkhan & Au skarn & $\mathrm{Au}, \mathrm{Cu}, \mathrm{Fe}$ & Gentral Mongolian \\
\hline $\mathrm{M}$ & 46 & 27 & Yolochka & $\mathrm{Cu}( \pm \mathrm{Fe}, \mathrm{Au}, \mathrm{Ag}, \mathrm{Mo})$ skarn & $\mathrm{Au}, \mathrm{Cu}, \mathrm{Fe}$ & Hovd \\
\hline $\mathrm{M}$ & 46 & 28 & Erdenekhairkhan & $\mathrm{Cu}( \pm \mathrm{Fe}, \mathrm{Au}, \mathrm{Ag}, \mathrm{Mo})$ skarn & $\mathrm{Au}, \mathrm{Cu}, \mathrm{Fe}$ & Central Mongolia \\
\hline $\mathrm{M}$ & 46 & 29 & Khagarlyn & $\mathrm{Cu}( \pm \mathrm{Fe}, \mathrm{Au}, \mathrm{Ag}, \mathrm{Mo})$ skarn & $\mathrm{Au}, \mathrm{Cu}$ & Hovd \\
\hline $\mathrm{M}$ & 46 & 3 & Tora-Sairskoye & Clastic sediment-hosted $\mathrm{Hg} \pm \mathrm{Sb}$ & $\mathrm{Hg}$ & Terligkhaisk \\
\hline $\mathrm{M}$ & 46 & 30 & Shartolgoi & Ta-Nb-REE alkaline metasomatite & $\mathrm{Ta}, \mathrm{Nb}, \mathrm{Zr}, \mathrm{Y}$ & Khalzanburged \\
\hline $\mathrm{M}$ & 46 & 31 & Nukhet & Carbonate-hosted Pb-Zn (Mississippi valley type) & $\mathrm{Zn}, \mathrm{Pb}$ & Central Mongolia \\
\hline $\mathrm{M}$ & 46 & 32 & Namiryn gol & Au-Ag epithermal vein & $\mathrm{Cu}$ & Uuregnuur \\
\hline $\mathrm{M}$ & 46 & 33 & Gozgor & Volcanogenic $\mathrm{Cu}-\mathrm{Zn}$ massive sulfide (Urals type) & $\mathrm{Cu}$ & Lake \\
\hline $\mathrm{M}$ & 46 & 34 & Achit nuur & W-Mo-Be greisen, stockwork, and quartz vein & $\mathrm{W}$ & Mongol Altai \\
\hline $\mathrm{M}$ & 46 & 35 & Kupol & Sediment-hosted $\mathrm{Cu}$ & $\mathrm{Cu}$ & Uuregnuur \\
\hline $\mathrm{M}$ & 46 & 36 & Otor uul & $\mathrm{Cu}( \pm \mathrm{Fe}, \mathrm{Au}, \mathrm{Ag}, \mathrm{Mo})$ skarn & $\mathrm{Cu}$ & Deluun-Sagsai \\
\hline $\mathrm{M}$ & 46 & 37 & Daltyn Khudag & Sn skarn & $\mathrm{Sn}$ & Deluun-Sagsai \\
\hline $\mathrm{M}$ & 46 & 38 & Omnogobi & Sediment-hosted $\mathrm{Cu}$ & $\mathrm{Cu}$ & Hovd \\
\hline $\mathrm{M}$ & 46 & 39 & Khatuugiin & Ta-Nb-REE alkaline metasomatite & REE, Zr, Ta & Mongolian-Altai \\
\hline $\mathrm{M}$ & 46 & 4 & Arzakskoye & Volcanic-hosted $\mathrm{Hg}$ & $\mathrm{Hg}$ & Terlighaisk \\
\hline $\mathrm{M}$ & 46 & 40 & Umnu Khutel & W-Mo-Be greisen, stockwork, and quartz vein & $\mathrm{W}, \mathrm{Mo}$ & Mongol Altai \\
\hline $\mathrm{M}$ & 46 & 41 & Dulaan khar uul & Ag-Pb epithermal vein & $\mathrm{Ag}, \mathrm{Pb}, \mathrm{Zn}$ & Deluun-Sagsai \\
\hline $\mathrm{M}$ & 46 & 42 & Bayanbulag & Sediment-hosted $\mathrm{Cu}$ & $\mathrm{Cu}$ & Deluun-Sagsai \\
\hline $\mathrm{M}$ & 46 & 43 & Khuren tolgoi & Volcanogenic $\mathrm{Cu}-\mathrm{Zn}$ massive sulfide (Urals type) & $\mathrm{Cu}$ & Lake \\
\hline $\mathrm{M}$ & 46 & 44 & Ulaan uul & W-Mo-Be greisen, stockwork, and quartz vein & $\mathrm{W}$ & Mongol Altai \\
\hline $\mathrm{M}$ & 46 & 45 & Molybdenum Stockwork & W-Mo-Be greisen, stockwork, and quartz vein & Mo, W & Mongol Altai \\
\hline $\mathrm{M}$ & 46 & 46 & Tsunkheg & W-Mo-Be greisen, stockwork, and quartz vein & $\mathrm{W}$ & Mongol Altai \\
\hline $\mathrm{M}$ & 46 & 47 & Maikhan Uul & Albite syenite-related REE & $\mathrm{Ta}, \mathrm{Nb}, \mathrm{REE}$ & Mongol Altai \\
\hline $\mathrm{M}$ & 46 & 48 & Tsakhir & Peralkaline granitoid-related Nb-Zr-REE & $\mathrm{Nb}, \mathrm{Ta}$ & Khalzanburged \\
\hline $\mathrm{M}$ & 46 & 49 & Khalzanburegtei & Ta-Nb-REE alkaline metasomatite & $\mathrm{Nb}, \mathrm{Zr}$ & Khalzanburged \\
\hline $\mathrm{M}$ & 46 & 5 & Akolskoye & $\mathrm{Ni}$-Co arsenide vein & $\mathrm{Ag}, \mathrm{Sb}$ & Khovuaksinsk \\
\hline $\mathrm{M}$ & 46 & 50 & Teht & $\mathrm{Ni}$-Co arsenide vein & $\mathrm{Co}$ & Kurai-Tolbo Nuur \\
\hline $\mathrm{M}$ & 46 & 51 & Khyargas & Granitoid-related $\mathrm{Au}$ vein & $\mathrm{Au}, \mathrm{Ag}, \mathrm{Cu}$ & Lake \\
\hline $\mathrm{M}$ & 46 & 51 & Sharbuureg & $\mathrm{Ag}$-Sb vein & $\mathrm{Ag}, \mathrm{Sb}$ & Kurai-Tolbo Nuur \\
\hline $\mathrm{M}$ & 46 & 52 & Khatuugiin gol & Sediment-hosted $\mathrm{Cu}$ & $\mathrm{Cu}$ & Deluun-Sagsai \\
\hline $\mathrm{M}$ & 46 & 53 & Tolbo nuur & Ag-Sb vein & $\mathrm{Ag}, \mathrm{Sb}$ & Kurai-Tolbo Nuur \\
\hline $\mathrm{M}$ & 46 & 54 & Tolbo & Au-Ag epithermal vein & $\mathrm{Cu}$ & Kurai-Tolbo Nuur \\
\hline M & 46 & 55 & Khukh-Adar & $\begin{array}{l}\text { Volcanogenic-hydrothermal-sedimentary massive sulfide } \\
\mathrm{Pb}-\mathrm{Zn}( \pm \mathrm{Cu})\end{array}$ & $\mathrm{Cu}, \mathrm{Zn}, \mathrm{Pb}$ & Uzuurtolgoi \\
\hline $\mathrm{M}$ & 46 & 56 & Baruun Tserd & Evaporate sedimentary gypsum & Gypsum & Unassigned \\
\hline $\mathrm{M}$ & 46 & 58 & Khargait 2 & Au-Ag epithermal vein & $\mathrm{Cu}$ & Uuregnuur \\
\hline M & 46 & 59 & Tsagaangol & Ni-Co arsenide vein & Co & Kurai-Tolbo Nuur \\
\hline $\mathrm{M}$ & 46 & 6 & Terligkhaiskoye & Volcanic-hosted Hg & $\mathrm{Hg}$ & Terligkhaisk \\
\hline $\mathrm{M}$ & 46 & 7 & Uzun-Oy & Ni-Co arsenide vein & $\mathrm{Co}, \mathrm{Cu}$ & Khovuaksinsk \\
\hline
\end{tabular}


\begin{tabular}{|l|l|l|l|l|l|l|}
\hline Row & Col. & No. & Deposit Name & Mineral Deposit Type & Major Metals & Metallogenic Belt \\
\hline
\end{tabular}

\begin{tabular}{|c|c|c|c|c|c|c|}
\hline $\mathrm{M}$ & 46 & 8 & Hovu-Aksinskoye & Ni-Co arsenide vein & $\mathrm{Co}, \mathrm{Ni}$ & Khovuaksinsk \\
\hline $\mathrm{M}$ & 46 & 9 & Aryskanskoye 2 & Banded iron formation (BIF, Superior Fe) & $\mathrm{Fe}$ & Mugursk \\
\hline M & 47 & 1 & Boksonskoye & Sedimentary bauxite & $\mathrm{Al}$ & Bokson-Kitoiskiy \\
\hline $\mathrm{M}$ & 47 & 10 & Berhem uul & Sedimentary phosphate & $\mathrm{P}$ & Hovsgol \\
\hline $\mathrm{M}$ & 47 & 11 & Khargana gol & Metamorphic graphite & Graphite & Unassigned \\
\hline $\mathrm{M}$ & 47 & 12 & Manhan uul & Sedimentary phosphate & $\mathrm{P}$ & Hovsgol \\
\hline $\mathrm{M}$ & 47 & 13 & Duchin gol & Magmatic nepheline & $\mathrm{Al}$ & Bugseingol-Ovormaraat \\
\hline $\mathrm{M}$ & 47 & 14 & Beltesin gol & Magmatic nepheline & $\mathrm{Al}$ & Bugseingol-Ovormaraat \\
\hline $\mathrm{M}$ & 47 & 15 & Uvurmaraat (Ujigin gol) & Magmatic nepheline & $\mathrm{Al}$ & Bugseingol-Ovormaraat \\
\hline $\mathrm{M}$ & 47 & 16 & Dalan & Sedimentary phosphate & $\mathrm{P}$ & Hovsgol \\
\hline $\mathrm{M}$ & 47 & 17 & Suultolgoi & Sedimentary phosphate & $\mathrm{P}$ & Hovsgol \\
\hline $\mathrm{M}$ & 47 & 18 & Altanboom & Ta-Nb-REE alkaline metasomatite & $\mathrm{Ta}$ & Bugseingol-Ovotmaraat \\
\hline M & 47 & 19 & Burenhan & Sedimentary phosphate & $\mathrm{P}$ & Hovsgol \\
\hline $\mathrm{M}$ & 47 & 2 & Uhagol & Sedimentary phosphate & $P$ & Hovsgol \\
\hline M & 47 & 20 & Hitagiin gol & Sedimentary Fe-V & $\mathrm{V}$ & Hovsgol \\
\hline M & 47 & 21 & Khanjargalant Uul & $\mathrm{Cu}( \pm \mathrm{Fe}, \mathrm{Au}, \mathrm{Ag}, \mathrm{Mo})$ skarn & $\mathrm{Cu}$ & Telmen \\
\hline $\mathrm{M}$ & 47 & 22 & Manganese & Volcanogenic-sedimentary Mn & $\mathrm{Mn}$ & H ovsgol \\
\hline $\mathrm{M}$ & 47 & 23 & Tsagaan Tolgoi & W-Mo-Be greisen, stockwork, and quartz vein & Mo & Unassigned \\
\hline $\mathrm{M}$ & 47 & 24 & Khoo Ulaan Uul & Porphyry $\mathrm{Cu}-\mathrm{Mo}( \pm \mathrm{Au}, \mathrm{Ag})$ & $\mathrm{Cu}$ & Orhon-Selenge \\
\hline $\mathrm{M}$ & 47 & 25 & Kuskunug & Serpentinite-hosted asbestos & $\begin{array}{l}\text { Chrysotile- } \\
\text { asbestos }\end{array}$ & Unassigned \\
\hline $\mathrm{M}$ & 47 & 26 & Agashskoye & Ta-Nb-REE alkaline metasomatite & $\mathrm{Ta}, \mathrm{Nb}, \mathrm{REE}$ & Ulug-Tanzek \\
\hline M & 47 & 27 & Bayan-Kol & Magmatic nepheline & $\mathrm{Al}$ & Bayan-Kol \\
\hline $\mathrm{M}$ & 47 & 28 & Kharlinskoye & Magmatic nepheline & $\mathrm{Al}$ & Bayan-Kol \\
\hline M & 47 & 29 & Verkhne-Kundusskoye & Ta-Nb-REE alkaline metasomatite & $\mathrm{Ta}, \mathrm{Nb}, \mathrm{REE}$ & Ulug-Tanzek \\
\hline $\mathrm{M}$ & 47 & 3 & Uliin davaa & Sedimentary phosphate & $\mathrm{P}$ & Hovsgol \\
\hline $\mathrm{M}$ & 47 & 30 & Dahu-Nurskoye & Magmatic nepheline & $\mathrm{Al}$ & Bayan-Kol \\
\hline $\mathrm{M}$ & 47 & 31 & Shukbulskoye & REE-Li pegmatite & $\mathrm{Li}$ & Tastyg \\
\hline M & 47 & 32 & Toskulskoye & Magmatic nepheline & $\mathrm{Al}$ & Bayan-Kol \\
\hline $\mathrm{M}$ & 47 & 33 & Zost tolgoi & Porphyry $\mathrm{Cu}-\mathrm{Mo}( \pm \mathrm{Au}, \mathrm{Ag})$ & $\mathrm{Cu}, \mathrm{Mo}$ & Orhon-Selenge \\
\hline $\mathrm{M}$ & 47 & 34 & Minjuurt tolgoi & $\mathrm{Cu}( \pm \mathrm{Fe}, \mathrm{Au}, \mathrm{Ag}, \mathrm{Mo})$ skarn & $\mathrm{Cu}$ & Telmen \\
\hline $\mathrm{M}$ & 47 & 35 & Pichi-Kholskoye & Magmatic nepheline & $\mathrm{Al}$ & Bayan-Kol \\
\hline $\mathrm{M}$ & 47 & 36 & Tsagaan-Uul & Sedimentary exhalative $\mathrm{Pb}-\mathrm{Zn}$ (SEDEX) & $\mathrm{Zn}, \mathrm{Pb}$ & Hugiingol \\
\hline $\mathrm{M}$ & 47 & 37 & Ulug-Tanzek & Ta-Nb-REE alkaline metasomatite & $\mathrm{Ta}, \mathrm{Nb}, \mathrm{REE}$ & Ulug-Tanzek \\
\hline $\mathrm{M}$ & 47 & 38 & Ulan-Erginskoye & Magmatic nepheline & $\mathrm{Al}$ & Bayan-Kol \\
\hline $\mathrm{M}$ & 47 & 39 & Korgere-Daba & Magmatic nepheline & $\mathrm{Al}$ & Bayan-Kol \\
\hline $\mathrm{M}$ & 47 & 4 & Hogorgo & Sedimentary phosphate & $\mathrm{P}$ & Hovsgol \\
\hline $\mathrm{M}$ & 47 & 40 & Kara-Adyr & REE-Li pegmatite & $\mathrm{Li}$ & Tastyg \\
\hline $\mathrm{M}$ & 47 & 41 & Chikskoye & Magmatic nepheline & $\mathrm{Al}$ & Bayan-Kol \\
\hline $\mathrm{M}$ & 47 & 42 & Verkhne-Emigenskoye & REE-Li pegmatite & $\mathrm{Li}$ & Tastyg \\
\hline $\mathrm{M}$ & 47 & 43 & Tastygskoye & REE-Li pegmatite & $\mathrm{Li}, \mathrm{Ta}, \mathrm{Nb}, \mathrm{Be}$ & Tastyg \\
\hline $\mathrm{M}$ & 47 & 44 & Bayangol 1 & REE-Li pegmatite & $\mathrm{Li}$ & Buteeliin nuruu \\
\hline $\mathrm{M}$ & 47 & 45 & Burginskoye & REE-Li pegmatite & $\mathrm{Li}$ & Tastyg \\
\hline $\mathrm{M}$ & 47 & 46 & Pichi-Tastygskoye & REE-Li pegmatite & $\mathrm{Li}$ & Tastyg \\
\hline $\mathrm{M}$ & 47 & 47 & Seveligskoye & REE-Li pegmatite & $\mathrm{Li}$ & Tastyg \\
\hline $\mathrm{M}$ & 47 & 48 & Khartynskoye & REE-Li pegmatite & $\mathrm{Li}$ & Tastyg \\
\hline $\mathrm{M}$ & 47 & 49 & Salbart group & Banded iron formation (BIF, Superior Fe) & $\mathrm{Fe}$ & Tarvagatai \\
\hline $\mathrm{M}$ & 47 & 5 & Khachim gol & Mafic-ultramafic related Ti-Fe (V) & $\mathrm{Fe}$ & Khachim gol \\
\hline $\mathrm{M}$ & 47 & 50 & Skarn & $\mathrm{Cu}( \pm \mathrm{Fe}, \mathrm{Au}, \mathrm{Ag}, \mathrm{Mo})$ skarn & $\mathrm{Cu}$ & Telmen \\
\hline $\mathrm{M}$ & 47 & 51 & Kharaat Uul & $\mathrm{Cu}( \pm \mathrm{Fe}, \mathrm{Au}, \mathrm{Ag}, \mathrm{Mo})$ skarn & $\mathrm{Cu}$ & Telmen \\
\hline
\end{tabular}




\begin{tabular}{|c|c|c|c|c|c|c|}
\hline $\mathrm{M}$ & 47 & 52 & Zos Uul & Porphyry $\mathrm{Cu}-\mathrm{Mo}( \pm \mathrm{Au}, \mathrm{Ag})$ & $\mathrm{Cu}, \mathrm{Mo}$ & Central Mongolia \\
\hline $\mathrm{M}$ & 47 & 53 & Onts Uul & Mafic-ultramafic related Cu-Ni-PGE & $\mathrm{Cu}$ & Telmen \\
\hline $\mathrm{M}$ & 47 & 54 & Naran bulag & Porphyry Cu-Mo $( \pm \mathrm{Au}, \mathrm{Ag})$ & $\mathrm{Cu}$ & Central Mongolia \\
\hline $\mathrm{M}$ & 47 & 55 & Solongot & $\mathrm{Cu}( \pm \mathrm{Fe}, \mathrm{Au}, \mathrm{Ag}, \mathrm{Mo})$ skarn & $\mathrm{Cu}$ & Telmen \\
\hline $\mathrm{M}$ & 47 & 56 & Most uul & Mafic-ultramafic related Ti-Fe (V) & $\mathrm{Fe}$ & Tarvagatai \\
\hline $\mathrm{M}$ & 47 & 57 & Takhilt nuur & Mafic-ultramafic related Cu-Ni-PGE & $\mathrm{Cu}, \mathrm{Ni}$ & Telmen \\
\hline $\mathrm{M}$ & 47 & 58 & Khagnuur & Volcanogenic-sedimentary $\mathrm{Mn}$ & $\mathrm{Mn}$ & Tsagaanolom \\
\hline $\mathrm{M}$ & 47 & 6 & Tsagaan Nuur & Sedimentary phosphate & $\mathrm{P}$ & Hovsgol \\
\hline M & 47 & 7 & Saihangol & Volcanogenic-sedimentary Mn & $\mathrm{Mn}$ & Hovsgol \\
\hline $\mathrm{M}$ & 47 & 8 & Hubsugul & Sedimentary phosphate & $\mathrm{P}$ & Hovsgol \\
\hline $\mathrm{M}$ & 47 & 9 & Bagatsagaan gol & Volcanogenic-sedimentary Mn & $\mathrm{Mn}$ & Hovsgol \\
\hline $\mathrm{M}$ & 48 & 1 & Oshurkovskoye & Magmatic and metasomatic apatite & Apatite & Dzhida-Selenginskiy \\
\hline M & 48 & 10 & Tarvagatai & Granitoid-related Au vein & $\mathrm{Au}, \mathrm{Ag}, \mathrm{Pb}, \mathrm{Zn}$ & Dzid-Selenginskiy \\
\hline $\mathrm{M}$ & 48 & 11 & Oyuut Uul & Porphyry $\mathrm{Cu}-\mathrm{Mo}( \pm \mathrm{Au}, \mathrm{Ag})$ & $\mathrm{Mo}, \mathrm{Cu}$ & Orhon-Selenge \\
\hline $\mathrm{M}$ & 48 & 12 & Bots & Basaltic $\mathrm{Cu}$ (Lake Superior type) & $\mathrm{Cu}$ & Orhon-Selenge \\
\hline M & 48 & 13 & Malo-Oinogorskoye & Porphyry Mo $( \pm \mathrm{W}, \mathrm{Sn}, \mathrm{Bi})$ & Mo & Dzhida-Selenginskiy \\
\hline $\mathrm{M}$ & 48 & 14 & Dzhidinskoe district & W-Mo-Be greisen, stockwork, and quartz vein & $\mathrm{W}$ & Dzhida-Selenginskiy \\
\hline $\mathrm{M}$ & 48 & 15 & Tomor tolgoi & Fe skarn & $\mathrm{Fe}$ & Bayangol \\
\hline $\mathrm{M}$ & 48 & 16 & Tavt & Granitoid-related Au vein & $\mathrm{Au}, \mathrm{Ag}, \mathrm{Cu}$ & Dzid-Selenginskiy \\
\hline $\mathrm{M}$ & 48 & 17 & Serten & $\mathrm{Cu}( \pm \mathrm{Fe}, \mathrm{Au}, \mathrm{Ag}, \mathrm{Mo})$ skarn & $\mathrm{Cu}$ & Bayangol \\
\hline $\mathrm{M}$ & 48 & 18 & Khotol & Mafic-ultramafic related $\mathrm{Cu}-\mathrm{Ni}$-PGE & $\mathrm{Ni}$ & Bayangol \\
\hline M & 48 & 19 & Baruunburen & Basaltic $\mathrm{Cu}$ (Lake Superior type) & $\mathrm{Cu}$ & Orhon-Selenge \\
\hline $\mathrm{M}$ & 48 & 2 & Arsentievskoye & Mafic-ultramafic related Ti-Fe (V) & $\mathrm{Ti}$ & Dzhida-Selenginskiy \\
\hline $\mathrm{M}$ & 48 & 20 & Urt & Granitoid-related Au vein & $\mathrm{Au}$ & North Hentii 2 \\
\hline $\mathrm{M}$ & 48 & 21 & Boroo 4 & Granitoid-related Au vein & $\mathrm{Au}$ & North Hentii 1 \\
\hline $\mathrm{M}$ & 48 & 22 & Boroo 7 & Granitoid-related Au vein & $\mathrm{Au}$ & North Hentii 2 \\
\hline $\mathrm{M}$ & 48 & 23 & Teshig 1 & Au skarn & $\mathrm{Au}$ & Dzid-Selenginskiy \\
\hline M & 48 & 24 & Boroo & Granitoid-related Au vein & $\mathrm{Au}$ & North Hentii 2 \\
\hline $\mathrm{M}$ & 48 & 25 & Baavgait & Granitoid-related $\mathrm{Au}$ vein & $\mathrm{Au}$ & North-Khentii \\
\hline $\mathrm{M}$ & 48 & 26 & Sujigt & Granitoid-related $\mathrm{Au}$ vein & $\mathrm{Au}$ & North Hentii 2 \\
\hline M & 48 & 27 & Serten-Nomgon & Mafic-ultramafic related Cu-Ni-PGE & $\mathrm{Cu}$ & Bayangol \\
\hline $\mathrm{M}$ & 48 & 28 & Tsagaanchuluut & Granitoid-related $\mathrm{Au}$ vein & $\mathrm{Au}$ & North Hentii 1 \\
\hline $\mathrm{M}$ & 48 & 29 & Narantolgoi & Granitoid-related Au vein & $\mathrm{Au}$ & North Hentii 2 \\
\hline M & 48 & 3 & Naranskoye & Fluospar vein & Fluorite & Dzhida-Selenginskiy \\
\hline $\mathrm{M}$ & 48 & 30 & Zalaat & Serpentinite-hosted asbestos & Asbestos & Egiingol \\
\hline $\mathrm{M}$ & 48 & 31 & Saikhan (Bor Khujir) & W-Mo-Be greisen, stockwork, and quartz vein & $\mathrm{W}, \mathrm{Sn}, \mathrm{Be}$ & Central Hentii \\
\hline M & 48 & 32 & Bayantsagaan 1 & Granitoid-related Au vein & $\mathrm{Au}$ & North Hentii 1 \\
\hline $\mathrm{M}$ & 48 & 33 & Erdenetiin Ovoo & Porphyry Cu-Mo $( \pm \mathrm{Au}, \mathrm{Ag})$ & $\mathrm{Cu}, \mathrm{Mo}$ & Orhon-Selenge \\
\hline $\mathrm{M}$ & 48 & 34 & Khujiryngol & Porphyry $\mathrm{Cu}( \pm \mathrm{Au})$ & $\mathrm{Cu}$ & Orhon-Selenge \\
\hline $\mathrm{M}$ & 48 & 35 & Tsagaan dabaa & W-Mo-Be greisen, stockwork, and quartz vein & W, Mo & Central Hentii \\
\hline $\mathrm{M}$ & 48 & 36 & Shand & Porphyry Cu-Mo $( \pm \mathrm{Au}, \mathrm{Ag})$ & $\mathrm{Cu}, \mathrm{Mo}$ & Orhon-Selenge \\
\hline $\mathrm{M}$ & 48 & 37 & Zuunturuu gol & Basaltic $\mathrm{Cu}$ (Lake Superior type) & $\mathrm{Cu}$ & Unassigned \\
\hline M & 48 & 38 & Bulgan & Basaltic $\mathrm{Cu}$ (Lake Superior type) & $\mathrm{Cu}$ & Orhon-Selenge \\
\hline $\mathrm{M}$ & 48 & 39 & Bumbat & $\mathrm{Au}$ in shear zone and quartz vein & $\mathrm{Au}$ & North Hentii 1 \\
\hline $\mathrm{M}$ & 48 & 4 & Baikalskoye & Banded iron formation (BIF, Superior Fe) & $\mathrm{Fe}$ & Sharizhalgaiskiy \\
\hline $\mathrm{M}$ & 48 & 40 & Ormiin Tsagaan nuur & Porphyry Cu-Mo $( \pm \mathrm{Au}, \mathrm{Ag})$ & $\mathrm{Cu}, \mathrm{Mo}$ & North Mongolian \\
\hline M & 48 & 41 & Nariin-gol & $\mathrm{Au}$ in shear zone and quartz vein & $\mathrm{Au}$ & North Hentii 1 \\
\hline $\mathrm{M}$ & 48 & 42 & Slyudyanskoe & Phlogopite skarn & Phlogopite & Prisayanskiy \\
\hline $\mathrm{M}$ & 48 & 5 & Ereen & Granitoid-related Au vein & $\mathrm{Au}$ & Dzid-Selenginskiy \\
\hline $\mathrm{M}$ & 48 & 6 & Tomortei & Fe skarn & $\mathrm{Fe}$ & Bayangol \\
\hline
\end{tabular}




\begin{tabular}{|c|c|c|c|c|c|c|}
\hline $\mathrm{M}$ & 48 & 7 & Bayangol 3 & Fe skarn & $\mathrm{Fe}$ & Bayangol \\
\hline $\mathrm{M}$ & 48 & 8 & Oyut tolgoi 3 & Fe skarn & $\mathrm{Fe}-\mathrm{Cu}-\mathrm{Au}$ & Bayangol \\
\hline $\mathrm{M}$ & 48 & 9 & Bulagtai & W-Mo-Be greisen, stockwork, and quartz vein & $\mathrm{W}, \mathrm{Mo}$ & Dzid-Selenginskiy \\
\hline $\mathrm{M}$ & 49 & 1 & Ara-Ilinskoe & Porphyry $\mathrm{Au}$ & $\mathrm{Au}$ & Onon-Turinskiy \\
\hline $\mathrm{M}$ & 49 & 10 & Lubavinskoye & Granitoid-related $\mathrm{Au}$ vein & $\mathrm{Au}$ & Onon-Turinskiy \\
\hline $\mathrm{M}$ & 49 & 11 & Gurvanbulag & Volcanic-hosted U & $\mathrm{U}$ & East Mongolian-Priargunskiy \\
\hline $\mathrm{M}$ & 49 & 12 & Baruunkhujirtyn gol & Au-Ag epithermal vein & $\mathrm{Au}$ & East Mongolian-Priargunskiy \\
\hline $\mathrm{M}$ & 49 & 13 & Shumilovskoe & Sn-W greisen, stockwork, and quartz vein & $\mathrm{W}$ & Onon-Chikoiskiy \\
\hline $\mathrm{M}$ & 49 & 14 & Kunaleiskoye & W-Mo-Be greisen, stockwork, and quartz vein & $\mathrm{W}$ & Onon-Chikoiskiy \\
\hline $\mathrm{M}$ & 49 & 15 & Delberkhei bulag & Granitoid-related Au vein & $\mathrm{Au}-\mathrm{Fe}$ & East Mongolian-Priargunskiy \\
\hline $\mathrm{M}$ & 49 & 16 & Narsan hundlun & Cassiterite-sulfide-silicate vein and stockwork & $\mathrm{Sn}$ & Eastern Mongolian-Preargunskiy \\
\hline $\mathrm{M}$ & 49 & 17 & Ikh-Khajuu & Sn-W greisen, stockwork, and quartz vein & Sn, W & Central Hentii \\
\hline $\mathrm{M}$ & 49 & 18 & Zuuntartsgol & Sn-W greisen, stockwork, and quartz vein & $\mathrm{W}, \mathrm{Sn}$ & Central Hentii \\
\hline $\mathrm{M}$ & 49 & 19 & Upper Kumyr & W-Mo-Be greisen, stockwork, and quartz vein & Sn, W & Onon-Chikoiskiy \\
\hline $\mathrm{M}$ & 49 & 2 & Bom-Gorhonskoye & W-Mo-Be greisen, stockwork, and quartz vein & $\mathrm{W}$ & Khilokskiy \\
\hline M & 49 & 20 & Bayanzurkh & Au-Ag epithermal vein & $\mathrm{Au}$ & East Mongolian-Priargunskiy \\
\hline $\mathrm{M}$ & 49 & 21 & Khavtgai & Fluospar vein & $\mathrm{CaF} 2$ & East Mongolian-Priargunskiy \\
\hline $\mathrm{M}$ & 49 & 3 & Tarbaldzheiskoe & Cassiterite-sulfide-silicate vein and stockwork & $\mathrm{Sn}$ & Onon-Turinskiy \\
\hline $\mathrm{M}$ & 49 & 4 & Tsagaanchuluut khudag II & Au-Ag epithermal vein & $\mathrm{Au}$ & East Mongolian-Priargunskiy \\
\hline $\mathrm{M}$ & 49 & 5 & Ugtam & $\mathrm{Au}-\mathrm{Ag}$ epithermal vein & $\mathrm{Au}$ & East Mongolian-Priargunskiy \\
\hline $\mathrm{M}$ & 49 & 6 & Khapcheranga & Cassiterite-sulfide-silicate vein and stockwork & $\mathrm{Sn}$ & Onon-Turinskiy \\
\hline $\mathrm{M}$ & 49 & 7 & Emtinbulag & Barite vein & $\mathrm{Ba}$ & East Mongolian-Priargunskiy \\
\hline $\mathrm{M}$ & 49 & 8 & Tsairyn & Au-Ag epithermal vein & $\mathrm{Au}$ & East Mongolian-Priargunskiy \\
\hline $\mathrm{M}$ & 49 & 9 & Bayandun & Fe-Zn skarn & $\mathrm{Zn}, \mathrm{Fe}$ & East Mongolian-Priargunskiy \\
\hline $\mathrm{M}$ & 50 & 1 & Novo-Shirokinskoye & Volcanic-hosted Au-base-metal metasomatite & $\mathrm{Pb}, \mathrm{Au}$ & East Mongolian-Priargunskiy \\
\hline $\mathrm{M}$ & 50 & 10 & Zapokrovskoye & Carbonate-hosted As-Au metasomatite & As & East Mongolian-Priargunskiy \\
\hline $\mathrm{M}$ & 50 & 11 & Gurulevskoe & Carbonate-hosted As-Au metasomatite & As & East Mongolian-Priargunskiy \\
\hline $\mathrm{M}$ & 50 & 12 & Oktjabrskoye & Carbonate-hosted As-Au metasomatite & As & East Mongolian-Priargunskiy \\
\hline $\mathrm{M}$ & 50 & 13 & Delmachik & Porphyry Au & $\mathrm{Au}$ & Shilkinsko-Tukuringrskiy \\
\hline $\mathrm{M}$ & 50 & 14 & Taseyevskoe & $\mathrm{Au}-\mathrm{Ag}$ epithermal vein & $\mathrm{Au}$ & Shilkinsko-Tukuringrskiy \\
\hline M & 50 & 15 & Baleyskoe & $\mathrm{Au}-\mathrm{Ag}$ epithermal vein & $\mathrm{Au}$ & Shilkinsko-Tukuringrskiy \\
\hline $\mathrm{M}$ & 50 & 16 & Andryushkinskoe & Au skarn & $\mathrm{Au}$ & Shilkinsko-Tukuringrskiy \\
\hline $\mathrm{M}$ & 50 & 17 & Bugdainskoye & W-Mo-Be greisen, stockwork, and quartz vein & Mo & East Mongolian-Priargunskiy \\
\hline $\mathrm{M}$ & 50 & 18 & $\begin{array}{l}\text { Xiaoyinuogaigou, Inner } \\
\text { Mongolia }\end{array}$ & Granitoid-related Au vein & $\mathrm{Au}$ & East Mongolian-Priargun-Derbugan \\
\hline $\mathrm{M}$ & 50 & 19 & Sredne-Golgotaiskoye & Granitoid-related $\mathrm{Au}$ vein & $\mathrm{Au}$ & Shilkinsko-Tukuringrskiy \\
\hline $\mathrm{M}$ & 50 & 2 & Solonechnoye & Fluospar vein & Fluorite & East Mongolian-Priargunskiy \\
\hline M & 50 & 20 & Severo-Akatuevskoye & Polymetallic $(\mathrm{Pb}, \mathrm{Zn}, \mathrm{Ag})$ carbonate-hosted metasomatite & $\mathrm{Pb}, \mathrm{Zn}$ & East Mongolian-Priargunskiy \\
\hline $\mathrm{M}$ & 50 & 21 & Akatuevskoye & Polymetallic $(\mathrm{Pb}, \mathrm{Zn}, \mathrm{Ag})$ carbonate-hosted metasomatite & $\mathrm{Pb}$ & East Mongolian-Priargunskiy \\
\hline $\mathrm{M}$ & 50 & 22 & Fatimovskoye & Granitoid-related $\mathrm{Au}$ vein & $\mathrm{Au}$ & Shilkinsko-Tukuringrskiy \\
\hline M & 50 & 23 & Zhetkovskoye & Fluospar vein & Fluorite & Shilkinsko-Tukuringrskiy \\
\hline $\mathrm{M}$ & 50 & 24 & Belukhinskoye & W-Mo-Be greisen, stockwork, and quartz vein & $\mathrm{W}$ & Shilkinsko-Tukuringrskiy \\
\hline $\mathrm{M}$ & 50 & 25 & Shunduinskoye & Granitoid-related Au vein & $\mathrm{Au}$ & Shilkinsko-Tukuringrskiy \\
\hline $\mathrm{M}$ & 50 & 26 & Bukukinskoye & W-Mo-Be greisen, stockwork, and quartz vein & $\mathrm{W}$ & Shilkinsko-Tukuringrskiy \\
\hline $\mathrm{M}$ & 50 & 27 & Etykinskoye & Ta-Nb-REE alkaline metasomatite & $\mathrm{Ta}$ & Shilkinsko-Tukuringrskiy \\
\hline $\mathrm{M}$ & 50 & 28 & Tamengskoye & Fluospar vein & Fluorite & Shilkinsko-Tukuringrskiy \\
\hline $\mathrm{M}$ & 50 & 29 & Kalanguyskoye & Fluospar vein & Fluorite & Shilkinsko-Tukuringrskiy \\
\hline $\mathrm{M}$ & 50 & 3 & Vozdvizhenskoye & Polymetallic $(\mathrm{Pb}, \mathrm{Zn}, \mathrm{Ag})$ carbonate-hosted metasomatite & $\mathrm{Pb}, \mathrm{Zn}$ & East Mongolian-Priargunskiy \\
\hline $\mathrm{M}$ & 50 & 30 & Savinskoye-5 & $\mathrm{Zn}-\mathrm{Pb}(\mathrm{Ag}, \mathrm{Cu}, \mathrm{W})$ skarn & $\mathrm{Pb}, \mathrm{Zn}$ & East Mongolian-Priargunskiy \\
\hline $\mathrm{M}$ & 50 & 31 & Liuyi, Inner Mongolia & $\begin{array}{l}\text { Volcanogenic-hydrothermal-sedimentary massive sulfide } \\
\mathrm{Pb}-\mathrm{Zn}( \pm \mathrm{Cu})\end{array}$ & $\mathrm{S}$ & Onor \\
\hline
\end{tabular}




\begin{tabular}{|c|c|c|c|c|c|c|}
\hline Row & Col. & No. & Deposit Name & Mineral Deposit Type & Major Metals & Metallogenic Belt \\
\hline $\mathrm{M}$ & 50 & 32 & Klichkinskoye & Polymetallic $(\mathrm{Pb}, \mathrm{Zn}, \mathrm{Ag})$ carbonate-hosted metasomatite & $\mathrm{Pb}$ & East Mongolian-Priargunskiy \\
\hline $\mathrm{M}$ & 50 & 33 & Barun-Shiveinsky & Hg-Sb-W vein and stockwork & $\mathrm{W}$ & Aginskiy \\
\hline $\mathrm{M}$ & 50 & 34 & Garsonuyskoye & Fluospar vein & Fluorite & East Mongolian-Priargunskiy \\
\hline $\mathrm{M}$ & 50 & 35 & $\begin{array}{l}\text { Badaguan, Inner } \\
\text { Mongolia }\end{array}$ & Porphyry Cu-Mo $( \pm \mathrm{Au}, \mathrm{Ag})$ & $\mathrm{Cu}$ & East Mongolian-Priargun-Derbugan \\
\hline $\mathrm{M}$ & 50 & 36 & Malo-Kulindinskoye & REE-Li pegmatite & $\mathrm{Ta}, \mathrm{Be}$ & Aginskiy \\
\hline M & 50 & 37 & Spokoininskoye & Sn-W greisen, stockwork, and quartz vein & $\mathrm{W}$ & Aginskiy \\
\hline $\mathrm{M}$ & 50 & 38 & Sherlovogorskoye & Cassiterite-sulfide-silicate vein and stockwork & Sn & Shilkinsko-Tukuringrskiy \\
\hline $\mathrm{M}$ & 50 & 39 & Orlovskoye & Ta-Nb-REE alkaline metasomatite & $\mathrm{Ta}$ & Aginskiy \\
\hline M & 50 & 4 & Berjozovskoe & Sedimentary siderite $\mathrm{Fe}$ & $\mathrm{Fe}$ & East Mongolian-Priargunskiy \\
\hline $\mathrm{M}$ & 50 & 40 & Abagaituyskoye & Fluospar vein & Fluorite & East Mongolian-Priargunskiy \\
\hline $\mathrm{M}$ & 50 & 41 & $\begin{array}{l}\text { Wunugetushan, Inner } \\
\text { Mongolia }\end{array}$ & Porphyry $\mathrm{Cu}-\mathrm{Mo}( \pm \mathrm{Au}, \mathrm{Ag})$ & $\mathrm{Cu}, \mathrm{Mo}$ & East Mongolian-Priargun-Derbugan \\
\hline $\mathrm{M}$ & 50 & 42 & Baits Ovoo & $\begin{array}{l}\text { Polymetallic }(\mathrm{Pb}, \mathrm{Zn} \pm \mathrm{Cu}, \mathrm{Ba}, \mathrm{Ag}, \mathrm{Au}) \text { volcanic-hosted } \\
\text { metasomatite }\end{array}$ & $\mathrm{Zn}, \mathrm{Pb}, \mathrm{Ag}$ & East Mongolian-Priargunskiy \\
\hline $\mathrm{M}$ & 50 & 43 & Berkh 2 & REE-Li pegmatite & $\mathrm{Ta}, \mathrm{Nb}$ & East Mongolian-Priargunskiy \\
\hline $\mathrm{M}$ & 50 & 44 & Ulaan & $\begin{array}{l}\text { Polymetallic }(\mathrm{Pb}, \mathrm{Zn} \pm \mathrm{Cu}, \mathrm{Ba}, \mathrm{Ag}, \mathrm{Au}) \text { volcanic-hosted } \\
\text { metasomatite }\end{array}$ & $\mathrm{Zn}, \mathrm{Pb}, \mathrm{Ag}$ & East Mongolian-Priargunskiy \\
\hline $\mathrm{M}$ & 50 & 45 & Khuts Ondor & Clastic-sediment-hosted Sb-Au & $\mathrm{Sb}$ & East Mongolian-Priargunskiy \\
\hline $\mathrm{M}$ & 50 & 46 & Bor Ondor & $\mathrm{Au}-\mathrm{Ag}$ epithermal vein & $\mathrm{Au}, \mathrm{Ag}$ & East Mongolian-Priargunskiy \\
\hline $\mathrm{M}$ & 50 & 47 & Avdartolgoi & Porphyry $\mathrm{Cu}-\mathrm{Mo}( \pm \mathrm{Au}, \mathrm{Ag})$ & $\mathrm{Cu}, \mathrm{Mo}$ & East Mongolian-Priargunskiy \\
\hline M & 50 & 48 & Ovorkhooloi & Granitoid-related $\mathrm{Au}$ vein & $\mathrm{Au}$ & East Mongolian-Priargunskiy \\
\hline M & 50 & 49 & Nomint & Granitoid-related $\mathrm{Au}$ vein & $\mathrm{Au}$ & East Mongolian-Priargunskiy \\
\hline $\mathrm{M}$ & 50 & 5 & Blagodatskoye & Polymetallic $(\mathrm{Pb}, \mathrm{Zn}, \mathrm{Ag})$ carbonate-hosted metasomatite & $\mathrm{Pb}$ & East Mongolian-Priargunskiy \\
\hline M & 50 & 50 & Chuluun Khoroot & W-Mo-Be greisen, stockwork, and quartz vein & $\mathrm{W}, \mathrm{Mo}$ & East Mongolian-Priargunskiy \\
\hline $\mathrm{M}$ & 50 & 51 & Erdenetolgoi & $\mathrm{Cu}( \pm \mathrm{Fe}, \mathrm{Au}, \mathrm{Ag}, \mathrm{Mo})$ skarn & $\mathrm{Au}-\mathrm{Cu}-\mathrm{Fe}$ & East Mongolian-Priargunskiy \\
\hline $\mathrm{M}$ & 50 & 52 & Jiawula, Inner Mongolia & $\begin{array}{l}\text { Polymetallic }(\mathrm{Pb}, \mathrm{Zn} \pm \mathrm{Cu}, \mathrm{Ba}, \mathrm{Ag}, \mathrm{Au}) \text { volcanic-hosted } \\
\text { metasomatite }\end{array}$ & $\mathrm{Ag}, \mathrm{Pb}, \mathrm{Zn}$ & East Mongolian-Priargun-Derbugan \\
\hline $\mathrm{M}$ & 50 & 53 & $\begin{array}{l}\text { Chaganbulagen, Inner } \\
\text { Mongolia }\end{array}$ & Au-Ag epithermal vein & $\mathrm{Ag}$ & East Mongolian-Priargunskiy- Deerbugan \\
\hline $\mathrm{M}$ & 50 & 54 & Zuun Dagai & Alkaline complex-hosted $\mathrm{Au}$ & $\mathrm{Au}, \mathrm{As}, \mathrm{Sb}, \mathrm{Te}$ & East Mongolian-Priargunskiy \\
\hline $\mathrm{M}$ & 50 & 55 & Kharguit & Granitoid-related Au vein & $\mathrm{Au}, \mathrm{As}, \mathrm{Sb}, \mathrm{Te}$ & East Mongolian-Priargunskiy \\
\hline $\mathrm{M}$ & 50 & 56 & Urliin Ovoo & Granitoid-related $\mathrm{Au}$ vein & $\mathrm{Au}, \mathrm{As}, \mathrm{Sb}, \mathrm{Te}$ & East Mongolian-Priargunskiy \\
\hline $\mathrm{M}$ & 50 & 57 & Bayan uul 1 & Granitoid-related $\mathrm{Au}$ vein & $\mathrm{Au}, \mathrm{Ag}, \mathrm{Pb}, \mathrm{Zn}$ & East Mongolian-Priargunskiy \\
\hline $\mathrm{M}$ & 50 & 58 & Tsav & Polymetallic $\mathrm{Pb}-\mathrm{Zn} \pm \mathrm{Cu}( \pm \mathrm{Ag}, \mathrm{Au})$ vein and stockwork & $\mathrm{Zn}, \mathrm{Pb}, \mathrm{Ag}$ & East Mongolian-Priargunskiy \\
\hline $\mathrm{M}$ & 50 & 59 & Dornod & Volcanic-hosted U & $\mathrm{U}$ & East Mongolian-Priargunskiy \\
\hline M & 50 & 6 & Kadainskoye & Polymetallic $(\mathrm{Pb}, \mathrm{Zn}, \mathrm{Ag})$ carbonate-hosted metasomatite & $\mathrm{Pb}, \mathrm{Zn}$ & East Mongolian-Priargunskiy \\
\hline $\mathrm{M}$ & 50 & 60 & $\begin{array}{l}\text { Erentaolegai, Inner } \\
\text { Mongolia }\end{array}$ & Au-Ag epithermal vein & $\mathrm{Ag}$ & East Mongolian-Priargunskiy- Deerbugan \\
\hline $\mathrm{M}$ & 50 & 61 & Baruunsuuj Undur & Fluospar vein & Fluorite & East Mongolian-Priargunskiy \\
\hline $\mathrm{M}$ & 50 & 62 & Khuvoobulag & Fluospar vein & Fluorite & East Mongolian-Priargunskiy \\
\hline $\mathrm{M}$ & 50 & 7 & Alenuiskoye & $\begin{array}{l}\text { Polymetallic }(\mathrm{Pb}, \mathrm{Zn} \pm \mathrm{Cu}, \mathrm{Ba}, \mathrm{Ag}, \mathrm{Au}) \text { volcanic-hosted } \\
\text { metasomatite }\end{array}$ & $\mathrm{Pb}$ & East Mongolian-Priargunskiy \\
\hline $\mathrm{M}$ & 50 & 8 & Shakhtaminskoye & Porphyry Mo $( \pm \mathrm{W}, \mathrm{Sn}, \mathrm{Bi})$ & Mo & East Mongolian-Priargunskiy \\
\hline $\mathrm{M}$ & 50 & 9 & Aprelkovskoye & Granitoid-related Au vein & $\mathrm{Au}$ & Shilkinsko-Tukuringrskiy \\
\hline $\mathrm{M}$ & 51 & 1 & Huanyu, Inner Mongolia & $\begin{array}{l}\text { Volcanogenic-hydrothermal-sedimentary massive sulfide } \\
\quad \mathrm{Pb}-\mathrm{Zn}( \pm \mathrm{Cu})\end{array}$ & $\mathrm{Pb} \mathrm{Zn}$ & Huanyu \\
\hline M & 51 & 2 & $\begin{array}{l}\text { Duobaoshan, } \\
\text { Heilongjiang Province }\end{array}$ & Porphyry Cu-Mo $( \pm \mathrm{Au}, \mathrm{Ag})$ & $\mathrm{Cu}$ & Duobaoshan \\
\hline $\mathrm{M}$ & 51 & 3 & $\begin{array}{l}\text { Tongshan, Heilongjiang } \\
\text { Province }\end{array}$ & Porphyry Cu-Mo $( \pm \mathrm{Au}, \mathrm{Ag})$ & $\mathrm{Cu}$ & Duobaoshan \\
\hline
\end{tabular}




\begin{tabular}{|c|c|c|c|c|c|c|}
\hline $\mathrm{M}$ & 51 & 4 & Sanhe, Inner Mongolia & $\begin{array}{l}\text { Polymetallic }(\mathrm{Pb}, \mathrm{Zn} \pm \mathrm{Cu}, \mathrm{Ba}, \mathrm{Ag}, \mathrm{Au}) \text { volcanic-hosted } \\
\text { metasomatite }\end{array}$ & $\mathrm{Pb}, \mathrm{Zn}$ & East Mongolian-Priargun (Derbugan) \\
\hline $\mathrm{M}$ & 51 & 5 & Xieertala, Inner Mongolia & Volcanogenic-sedimentary Fe & $\mathrm{Fe}$ & Onor \\
\hline $\mathrm{M}$ & 52 & 10 & $\begin{array}{l}\text { Bidzhanskoe } \\
\text { (Kabalinskoe) }\end{array}$ & Volcanogenic-sedimentary Mn & $\mathrm{Mn}$ & South Khingan \\
\hline $\mathrm{M}$ & 52 & 11 & Verkhnebidzhanskoe & Sn-W greisen, stockwork, and quartz vein & Sn & Malokhingan \\
\hline $\mathrm{M}$ & 52 & 12 & Kostenginskoe & Banded iron formation (BIF, Superior Fe) & $\mathrm{Fe}$ & South Khingan \\
\hline $\mathrm{M}$ & 52 & 13 & Preobrazhenovskoye & Fluorite greisen & Fluorite & Bidzhan \\
\hline $\mathrm{M}$ & 52 & 14 & $\begin{array}{l}\text { Pengdingshan, } \\
\text { Heilongjiang Province }\end{array}$ & Granitoid-related Au vein & $\mathrm{Au}$ & Tuanjiegou \\
\hline $\mathrm{M}$ & 52 & 15 & $\begin{array}{l}\text { Tuanjiegou, Heilongjiang } \\
\text { Province }\end{array}$ & Granitoid-related $\mathrm{Au}$ vein & $\mathrm{Au}$ & Tuanjiegou \\
\hline $\mathrm{M}$ & 52 & 16 & $\begin{array}{l}\text { Chuihongshan, } \\
\text { Heilongjiang Province }\end{array}$ & Fe-Zn skarn & $\mathrm{Fe}, \mathrm{W}, \mathrm{Mo}, \mathrm{Zn}$ & Bindong \\
\hline $\mathrm{M}$ & 52 & 17 & $\begin{array}{l}\text { Wuxing, Heilongjiang } \\
\text { Province }\end{array}$ & Mafic-ultramafic related $\mathrm{Cu}-\mathrm{Ni}$-PGE & $\mathrm{Pt}, \mathrm{Pd}$ & Wuxing \\
\hline $\mathrm{M}$ & 52 & 2 & Melginskoye & Porphyry Mo $( \pm \mathrm{W}, \mathrm{Sn}, \mathrm{Bi})$ & Mo & Melgin-Niman \\
\hline $\mathrm{M}$ & 52 & 3 & Chergilen & Felsic plutonic U-REE & REE, Be & Melgin-Niman \\
\hline $\mathrm{M}$ & 52 & 4 & Dzhalinda & Rhyolite-hosted Sn & $\mathrm{Sn}$ & Malo-Khingan \\
\hline $\mathrm{M}$ & 52 & 5 & Kimkanskoe & Banded iron formation (BIF, Superior Fe) & $\mathrm{Fe}$ & South Khingan \\
\hline $\mathrm{M}$ & 52 & 6 & Diturskoe & Felsic plutonic U-REE & REE & Bidzhan \\
\hline $\mathrm{M}$ & 52 & 7 & Khingan & Sn-W greisen, stockwork, and quartz vein & Sn & Malo-Khingan \\
\hline $\mathrm{M}$ & 52 & 8 & Sutarskoye & Banded iron formation (BIF, Superior Fe) & $\mathrm{Fe}$ & South Khingan \\
\hline $\mathrm{M}$ & 52 & 9 & Yuzhno-Khingan & Banded iron formation (BIF, Superior Fe) & $\mathrm{Fe}$ & South Khingan \\
\hline $\mathrm{M}$ & 53 & 1 & Birandzha & Porphyry Mo $( \pm \mathrm{W}, \mathrm{Sn}, \mathrm{Bi})$ & Mo & Ezop-Yam-Alin \\
\hline $\mathrm{M}$ & 53 & 10 & Boltoro & $\mathrm{Cu}( \pm \mathrm{Fe}, \mathrm{Au}, \mathrm{Ag}, \mathrm{Mo})$ skarn & $\mathrm{Sn}, \mathrm{Cu}$ & Badzhal-Komsomolsk \\
\hline $\mathrm{M}$ & 53 & 11 & Noni & $\mathrm{Au}-\mathrm{Ag}$ epithermal vein & $\mathrm{Au}$ & East Mongolian or Priargunskiy - ? \\
\hline $\mathrm{M}$ & 53 & 12 & Durmin & Au-Ag epithermal vein & $\mathrm{Au}, \mathrm{Ag}$ & Durmin \\
\hline $\mathrm{M}$ & 53 & 2 & Solnechnoe & Sn-W greisen, stockwork, and quartz vein & $\mathrm{Sn}$ & Badzhal-Komsomolsk \\
\hline M & 53 & 3 & Sobolinoye & Sn-W greisen, stockwork, and quartz vein & Sn & Badzhal-Komsomolsk \\
\hline $\mathrm{M}$ & 53 & 4 & Ippatinskoe & Sn-W greisen, stockwork, and quartz vein & Sn & Ezop-Yam-Alin \\
\hline $\mathrm{M}$ & 53 & 5 & Festivalnoe & Sn-W greisen, stockwork, and quartz vein & Sn & Badzhal-Komsomolsk \\
\hline $\mathrm{M}$ & 53 & 6 & Metrekskoye & Porphyry Mo $( \pm \mathrm{W}, \mathrm{Sn}, \mathrm{Bi})$ & Mo & Melgin-Niman \\
\hline $\mathrm{M}$ & 53 & 7 & Kapral & Porphyry Mo $( \pm \mathrm{W}, \mathrm{Sn}, \mathrm{Bi})$ & Mo & Badzhal-Komsomolsk \\
\hline $\mathrm{M}$ & 53 & 8 & Loshadinayagriva (Main) & Sn-W greisen, stockwork, and quartz vein & $\mathrm{Sn}$ & Badzhal-Komsomolsk \\
\hline $\mathrm{M}$ & 53 & 9 & Pravourmiiskoe & Sn-W greisen, stockwork, and quartz vein & $\mathrm{Sn}$ & Badzhal-Komsomolsk \\
\hline $\mathrm{M}$ & 54 & 1 & Agnie-Afanas'evskoye & Granitoid-related $\mathrm{Au}$ vein & $\mathrm{Au}$ & Pilda-Limuri \\
\hline $\mathrm{M}$ & 54 & 2 & Dyappe & $\mathrm{Ag}-\mathrm{Sb}$ vein & $\mathrm{Au}$ & Pilda-limuri \\
\hline $\mathrm{M}$ & 54 & 3 & Uchaminskoye & Polymetallic $\mathrm{Pb}-\mathrm{Zn} \pm \mathrm{Cu}( \pm \mathrm{Ag}, \mathrm{Au})$ vein and stockwork & $\mathrm{Au}$ & Pilda-Limuri \\
\hline $\mathrm{M}$ & 54 & 4 & Tumninskoye & $\mathrm{Au}-\mathrm{Ag}$ epithermal vein & $\mathrm{Au}$ & Tumnin-Anyuy \\
\hline $\mathrm{M}$ & 54 & 5 & Mopau & Porphyry Sn & $\mathrm{Sn}$ & Tumnin-Anyuy \\
\hline $\mathrm{M}$ & 54 & 6 & Nochnoe & Porphyry $\mathrm{Cu}( \pm \mathrm{Au})$ & $\mathrm{Cu}$ & Kema \\
\hline $\mathrm{M}$ & 54 & 7 & Moinskoe & Porphyry Mo $( \pm \mathrm{W}, \mathrm{Sn}, \mathrm{Bi})$ & Mo & Kema \\
\hline $\mathrm{M}$ & 54 & 8 & Sukhoi Creek & Porphyry $\mathrm{Cu}-\mathrm{Mo}( \pm \mathrm{Au}, \mathrm{Ag})$ & $\mathrm{Cu}, \mathrm{Mo}$ & Kema \\
\hline $\mathrm{N}$ & 44 & 1 & Kolyvanskoye & Sn-W greisen, stockwork, and quartz vein & Sn & Barlaksk \\
\hline $\mathrm{N}$ & 45 & 1 & Barandatskoye & Sedimentary siderite $\mathrm{Fe}$ & $\mathrm{Fe}$ & Unassigned \\
\hline $\mathrm{N}$ & 45 & 10 & Gavrilovskoye & Granitoid-related $\mathrm{Au}$ vein & $\mathrm{Au}$ & Martaiginsk \\
\hline $\mathrm{N}$ & 45 & 11 & Kurgusulskoye & Magmatic nepheline & $\mathrm{Al}$ & Kiya-Shaltyr \\
\hline $\mathrm{N}$ & 45 & 12 & Tulujul & Magmatic nepheline & $\mathrm{Al}$ & Kiya-Shaltyr \\
\hline $\mathrm{N}$ & 45 & 13 & Belogorskoye & Magmatic nepheline & $\mathrm{Al}$ & Kiya-Shaltyr \\
\hline $\mathrm{N}$ & 45 & 14 & Barzasskoye & Bauxite (karst type) & $\mathrm{Al}$ & Belininsk \\
\hline
\end{tabular}




\begin{tabular}{|c|c|c|c|c|c|c|}
\hline Row & Col. & No. & Deposit Name & Mineral Deposit Type & Major Metals & Metallogenic Belt \\
\hline $\mathrm{N}$ & 45 & 15 & Fedotovskoye & Granitoid-related $\mathrm{Au}$ vein & $\mathrm{Au}$ & Martaiginsk \\
\hline $\mathrm{N}$ & 45 & 16 & Kiya-Shaltyr & Magmatic nepheline & $\mathrm{Al}$ & Kiya-Shaltyr \\
\hline $\mathrm{N}$ & 45 & 17 & Centralnoye & Granitoid-related $\mathrm{Au}$ vein & $\mathrm{Au}$ & Martaiginsk \\
\hline $\mathrm{N}$ & 45 & 18 & Malorastaiskoye & Fluospar vein & Fluorite & Unassigned \\
\hline $\mathrm{N}$ & 45 & 19 & Ampalyk & Fe skarn & $\mathrm{Fe}$ & Taidon-Kondomsk \\
\hline $\mathrm{N}$ & 45 & 2 & Ust-Parninskoye & Polymetallic $\mathrm{Pb}-\mathrm{Zn} \pm \mathrm{Cu}( \pm \mathrm{Ag}, \mathrm{Au})$ vein and stockwork & $\mathrm{Pb}, \mathrm{Zn}$ & Sorsk \\
\hline $\mathrm{N}$ & 45 & 20 & Agaskyrskoye & Porphyry Mo $( \pm \mathrm{W}, \mathrm{Sn}, \mathrm{Bi})$ & Mo & Sorsk \\
\hline $\mathrm{N}$ & 45 & 21 & Gromotukhinskoye & Granitoid-related $\mathrm{Au}$ vein & $\mathrm{Au}$ & Martaiginsk \\
\hline $\mathrm{N}$ & 45 & 22 & Tuim & W-Mo-Be greisen, stockwork, and quartz vein & $\mathrm{W}$ & Kiyalykh-Uzen \\
\hline $\mathrm{N}$ & 45 & 23 & Sarala & Granitoid-related $\mathrm{Au}$ vein & $\mathrm{Au}$ & Martaiginsk \\
\hline $\mathrm{N}$ & 45 & 24 & Belo-Osipovskoye & Volcanic-hosted Hg & $\mathrm{Hg}$ & Kuznetsk \\
\hline $\mathrm{N}$ & 45 & 25 & Ipchulskoye & Porphyry Mo $( \pm \mathrm{W}, \mathrm{Sn}, \mathrm{Bi})$ & Mo & Sorsk \\
\hline $\mathrm{N}$ & 45 & 26 & Kiyalykh-Uzen & $\mathrm{Cu}( \pm \mathrm{Fe}, \mathrm{Au}, \mathrm{Ag}, \mathrm{Mo})$ skarn & $\mathrm{Cu}, \mathrm{Mo}$ & Kiyalykh-Uzen \\
\hline $\mathrm{N}$ & 45 & 27 & Kommunar & Granitoid-related $\mathrm{Au}$ vein & $\mathrm{Au}$ & Martaiginsk \\
\hline $\mathrm{N}$ & 45 & 28 & Kupriyanovskoye & Volcanic-hosted Hg & $\mathrm{Hg}$ & Kuznetsk \\
\hline $\mathrm{N}$ & 45 & 29 & Glafirinskoye & $\mathrm{Cu}( \pm \mathrm{Fe}, \mathrm{Au}, \mathrm{Ag}, \mathrm{Mo})$ skarn & $\mathrm{Cu}, \mathrm{Mo}$ & Kiyalykh-Uzen \\
\hline $\mathrm{N}$ & 45 & 3 & Natal'evskoye & Au skarn & $\mathrm{Au}$ & Martaiginsk \\
\hline $\mathrm{N}$ & 45 & 30 & Pezass & Carbonate-hosted $\mathrm{Hg}$-Sb & $\mathrm{Hg}$ & Kuznetsk \\
\hline $\mathrm{N}$ & 45 & 31 & Spasskoye & W-Mo-Be greisen, stockwork, and quartz vein & $\mathrm{W}$ & Kiyalykh-Uzen \\
\hline $\mathrm{N}$ & 45 & 32 & Balakhchino & Granitoid-related $\mathrm{Au}$ vein & $\mathrm{Au}$ & Martaiginsk \\
\hline $\mathrm{N}$ & 45 & 33 & Nichkuryupskoye & Porphyry Mo $( \pm \mathrm{W}, \mathrm{Sn}, \mathrm{Bi})$ & Mo & Sorsk \\
\hline $\mathrm{N}$ & 45 & 34 & Pezasskoye & Volcanic-hosted zeolite & Zeolite & Kuznetsk \\
\hline $\mathrm{N}$ & 45 & 35 & Turtek & W-Mo-Be greisen, stockwork, and quartz vein & $\mathrm{W}$ & Kiyalykh-Uzen \\
\hline $\mathrm{N}$ & 45 & 36 & Kayvinskoye & W-Mo-Be greisen, stockwork, and quartz vein & $\mathrm{W}$ & Kiyalykh-Uzen \\
\hline $\mathrm{N}$ & 45 & 37 & Verhne-Askizskoye & W-Mo-Be greisen, stockwork, and quartz vein & $\mathrm{W}$ & Kiyalykh-Uzen \\
\hline $\mathrm{N}$ & 45 & 39 & Lavrenovskoye & Fe skarn & $\mathrm{Fe}$ & Taidon-Kondomsk \\
\hline $\mathrm{N}$ & 45 & 4 & Komsomolskoye & Granitoid-related Au vein & $\mathrm{Au}$ & Martaiginsk \\
\hline $\mathrm{N}$ & 45 & 40 & Ityuiskoye & $\mathrm{Cu}( \pm \mathrm{Fe}, \mathrm{Au}, \mathrm{Ag}, \mathrm{Mo})$ skarn & $\mathrm{Cu}, \mathrm{Mo}$ & Kiyalykh-Uzen \\
\hline $\mathrm{N}$ & 45 & 41 & Usinskoye & Volcanogenic-sedimentary Mn & $\mathrm{Mn}$ & Taidon-Kondomsk \\
\hline $\mathrm{N}$ & 45 & 42 & Alguiskoye & Talc (magnesite) replacement & Talc & Unassigned \\
\hline $\mathrm{N}$ & 45 & 43 & Balyksa & $\mathrm{Cu}( \pm \mathrm{Fe}, \mathrm{Au}, \mathrm{Ag}, \mathrm{Mo})$ skarn & Mo & Kiyalykh-Uzen \\
\hline $\mathrm{N}$ & 45 & 44 & Kazymchanskoye & Polymetallic $(\mathrm{Pb}, \mathrm{Zn}, \mathrm{Ag})$ carbonate-hosted metasomatite & $\mathrm{Pb}, \mathrm{Zn}$ & Sorsk \\
\hline $\mathrm{N}$ & 45 & 45 & Sukharinskoye & Fe skarn & $\mathrm{Fe}$ & Taidon-Kondomsk \\
\hline $\mathrm{N}$ & 45 & 46 & Vaganovskoye & Bauxite (karst type) & $\mathrm{Al}$ & Belininsk \\
\hline $\mathrm{N}$ & 45 & 47 & Uskandinskoye & $\begin{array}{l}\text { Polymetallic }(\mathrm{Pb}, \mathrm{Zn} \pm \mathrm{Cu}, \mathrm{Ba}, \mathrm{Ag}, \mathrm{Au}) \text { volcanic-hosted } \\
\text { metasomatite }\end{array}$ & $\mathrm{Cu}, \mathrm{Zn}$ & Salair \\
\hline $\mathrm{N}$ & 45 & 48 & Urskoye district & $\begin{array}{l}\text { Polymetallic }(\mathrm{Pb}, \mathrm{Zn} \pm \mathrm{Cu}, \mathrm{Ba}, \mathrm{Ag}, \mathrm{Au}) \text { volcanic-hosted } \\
\text { metasomatite }\end{array}$ & $\mathrm{Pb}, \mathrm{Zn}, \mathrm{Cu}$ & Salair \\
\hline $\mathrm{N}$ & 45 & 49 & Elgentagskoye & Fe skarn & $\mathrm{Fe}$ & Teisk \\
\hline $\mathrm{N}$ & 45 & 5 & Goriachegorskoye & Magmatic nepheline & $\mathrm{Al}$ & Kiya-Shaltyr \\
\hline $\mathrm{N}$ & 45 & 50 & Teiskoye & Fe skarn & $\mathrm{Fe}$ & Teisk \\
\hline $\mathrm{N}$ & 45 & 51 & Abagasskoye & Fe skarn & $\mathrm{Fe}$ & Teisk \\
\hline $\mathrm{N}$ & 45 & 52 & Kamenushinskoye & Porphyry Cu-Mo $( \pm \mathrm{Au}, \mathrm{Ag})$ & $\mathrm{Cu}$ & Salair \\
\hline $\mathrm{N}$ & 45 & 53 & Izykhgolskoye & Fe skarn & $\mathrm{Fe}$ & Teisk \\
\hline $\mathrm{N}$ & 45 & 54 & Mavrinskoye & Clastic sediment-hosted $\mathrm{Hg} \pm \mathrm{Sb}$ & $\mathrm{Hg}$ & Mavrinsk \\
\hline $\mathrm{N}$ & 45 & 55 & Salairskoye & $\begin{array}{l}\text { Polymetallic }(\mathrm{Pb}, \mathrm{Zn} \pm \mathrm{Cu}, \mathrm{Ba}, \mathrm{Ag}, \mathrm{Au}) \text { volcanic-hosted } \\
\text { metasomatite }\end{array}$ & $\mathrm{Pb}, \mathrm{Zn}, \mathrm{Ag}, \mathrm{Au}$ & Salair \\
\hline $\mathrm{N}$ & 45 & 56 & Oktyabrskoye 4 & Bauxite (karst type) & $\mathrm{Al}$ & Berdsko-Maisk \\
\hline $\mathrm{N}$ & 45 & 57 & Novogodneye & Bauxite (karst type) & $\mathrm{Al}$ & Berdsko-Maisk \\
\hline $\mathrm{N}$ & 45 & 58 & Durnovskoye & Volcanogenic-sedimentary $\mathrm{Mn}$ & $\mathrm{Mn}$ & Unassigned \\
\hline $\mathrm{N}$ & 45 & 59 & Khaileolovskoye & Fe skarn & $\mathrm{Fe}$ & Teisk \\
\hline
\end{tabular}




\begin{tabular}{|c|c|c|c|c|c|c|}
\hline Row & Col. & No. & Deposit Name & Mineral Deposit Type & Major Metals & Metallogenic Belt \\
\hline $\mathrm{N}$ & 45 & 6 & Andrushkina River & Magmatic nepheline & $\mathrm{Al}$ & Kiya-Shaltyr \\
\hline $\mathrm{N}$ & 45 & 60 & Orlinogorskoye & Clastic sediment-hosted $\mathrm{Hg} \pm \mathrm{Sb}$ & $\mathrm{Hg}$ & Mavrinsk \\
\hline $\mathrm{N}$ & 45 & 61 & Berdsko-Maiskoye & Sedimentary bauxite & $\mathrm{Al}$ & Berdsko-Maisk \\
\hline $\mathrm{N}$ & 45 & 62 & Patynskoye & Mafic-ultramafic related Ti-Fe (V) & $\mathrm{Fe}, \mathrm{Ti}$ & Teisk \\
\hline $\mathrm{N}$ & 45 & 63 & Tashelginskoye & Fe skarn & $\mathrm{Fe}$ & Taidon-Kondomsk \\
\hline $\mathrm{N}$ & 45 & 64 & Chilanskoye & Volcanogenic-sedimentary $\mathrm{Fe}$ & $\mathrm{Fe}$ & Teisk \\
\hline $\mathrm{N}$ & 45 & 65 & Togulenskoye & Talc (magnesite) replacement & Talc & Belininsk \\
\hline $\mathrm{N}$ & 45 & 66 & Tamalykskoye & Sedimentary phosphate & Phosphorite & Mrass \\
\hline $\mathrm{N}$ & 45 & 67 & Svetlyi Klyuch & Talc (magnesite) replacement & Talc & Belininsk \\
\hline $\mathrm{N}$ & 45 & 68 & Obukhovskoye & Bauxite (karst type) & $\mathrm{Al}$ & Berdsko-Maisk \\
\hline $\mathrm{N}$ & 45 & 69 & Kedrovskoye & $\begin{array}{l}\text { Polymetallic }(\mathrm{Pb}, \mathrm{Zn} \pm \mathrm{Cu}, \mathrm{Ba}, \mathrm{Ag}, \mathrm{Au}) \text { volcanic-hosted } \\
\text { metasomatite }\end{array}$ & $\mathrm{Pb}, \mathrm{Zn}$ & Taidon-Kondomsk \\
\hline $\mathrm{N}$ & 45 & 7 & Staro-Berikul & Granitoid related $\mathrm{Au}$ vein & $\mathrm{Au}$ & Martaiginsk \\
\hline $\mathrm{N}$ & 45 & 70 & Telbes & Fe skarn & $\mathrm{Fe}$ & Taidon-Kondomsk \\
\hline $\mathrm{N}$ & 45 & 71 & Odrabash & Fe skarn & $\mathrm{Fe}$ & Taidon-Kondomsk \\
\hline $\mathrm{N}$ & 45 & 72 & Kul-Taiga & Mafic-ultramafic related Ti-Fe (V) & $\mathrm{Fe}, \mathrm{Ti}$ & Teisk \\
\hline $\mathrm{N}$ & 45 & 73 & Pykhtun & Fe skarn & $\mathrm{Fe}$ & Taidon-Kondomsk \\
\hline $\mathrm{N}$ & 45 & 74 & Kazskoye & Fe skarn & $\mathrm{Fe}$ & Taidon-Kondomsk \\
\hline $\mathrm{N}$ & 45 & 75 & Temirtau & Fe skarn & $\mathrm{Fe}$ & Taidon-Kondomsk \\
\hline $\mathrm{N}$ & 45 & 76 & Turgenevskoye & $\begin{array}{l}\text { Polymetallic }(\mathrm{Pb}, \mathrm{Zn} \pm \mathrm{Cu}, \mathrm{Ba}, \mathrm{Ag}, \mathrm{Au}) \text { volcanic-hosted } \\
\text { metasomatite }\end{array}$ & $\mathrm{Pb}, \mathrm{Zn}$ & Taidon-Kondomsk \\
\hline $\mathrm{N}$ & 45 & 77 & Belkinskoye & Sedimentary phosphate & Phosphorite & Mrass \\
\hline $\mathrm{N}$ & 45 & 78 & Volshebnaya Zhila & Granitoid-related Au vein & $\mathrm{Au}$ & Unassigned \\
\hline $\mathrm{N}$ & 45 & 79 & Semeno-Krasilovskoye & Sedimentary bauxite & $\mathrm{Al}$ & Belininsk \\
\hline $\mathrm{N}$ & 45 & 8 & Novo-Berikul & Granitoid-related $\mathrm{Au}$ vein & $\mathrm{Au}$ & Martaiginsk \\
\hline $\mathrm{N}$ & 45 & 80 & Taymetskoye & Basaltic $\mathrm{Cu}$ (Lake Superior type) & $\mathrm{Cu}$ & Taidon-Kondomsk \\
\hline $\mathrm{N}$ & 45 & 81 & Anzass & Fe skarn & $\mathrm{Fe}$ & North-Sayanian \\
\hline $\mathrm{N}$ & 45 & 82 & Belininskoye & Laterite $\mathrm{Ni}$ & $\mathrm{Ni}$ & Belininsk \\
\hline $\mathrm{N}$ & 45 & 83 & Sheregesh & Fe skarn & $\mathrm{Fe}$ & Taidon-Kondomsk \\
\hline $\mathrm{N}$ & 45 & 84 & Alexandrovskoye 2 & Laterite $\mathrm{Ni}$ & $\mathrm{Ni}$ & Belininsk \\
\hline $\mathrm{N}$ & 45 & 85 & Shalym & Fe skarn & $\mathrm{Fe}$ & Taidon-Kondomsk \\
\hline $\mathrm{N}$ & 45 & 86 & Tashtagol & Fe skarn & $\mathrm{Fe}$ & Taidon-Kondonskiy \\
\hline $\mathrm{N}$ & 45 & 87 & Kharadzulskoye & $\mathrm{Ni}-\mathrm{Co}$ arsenide vein & $\mathrm{Cu}, \mathrm{Co}$ & Kharadzhulsk \\
\hline $\mathrm{N}$ & 45 & 88 & Kayanchinskoye & Fluospar vein & Fluorite & Sarasinsk \\
\hline $\mathrm{N}$ & 45 & 9 & Kundatskoye & Granitoid-related $\mathrm{Au}$ vein & $\mathrm{Au}$ & Martaiginsk \\
\hline $\mathrm{N}$ & 46 & 1 & Irbinskoye & Fe skarn & $\mathrm{Fe}$ & Kizir-Kazyr \\
\hline $\mathrm{N}$ & 46 & 10 & Beryozovskoye & Fe skarn & $\mathrm{Fe}$ & Kizir-Kazyr \\
\hline $\mathrm{N}$ & 46 & 11 & Lysanskoye & Mafic-ultramafic related Ti-Fe (V) & $\mathrm{Ti}, \mathrm{Fe}$ & Lysansk \\
\hline $\mathrm{N}$ & 46 & 12 & Kedranskoye & Zoned mafic-ultramafic Cr-PGE & $\mathrm{Ti}, \mathrm{Fe}$ & Lysansk \\
\hline $\mathrm{N}$ & 46 & 13 & Pionerskoye 1 & Porphyry Mo $( \pm \mathrm{W}, \mathrm{Sn}, \mathrm{Bi})$ & Mo & Agulsk \\
\hline $\mathrm{N}$ & 46 & 14 & Dzhetskoye & Porphyry Mo $( \pm \mathrm{W}, \mathrm{Sn}, \mathrm{Bi})$ & Mo & Agulsk \\
\hline $\mathrm{N}$ & 46 & 15 & Karaulnaya Gorka & Weathering crust $\mathrm{Mn}( \pm \mathrm{Fe})$ & $\mathrm{Mn}$ & Djotsk \\
\hline $\mathrm{N}$ & 46 & 16 & Seibinskoye 1 & Weathering crust $\mathrm{Mn}( \pm \mathrm{Fe})$ & $\mathrm{Mn}$ & Djotsk \\
\hline $\mathrm{N}$ & 46 & 17 & Djotskoye & Weathering crust $\mathrm{Mn}( \pm \mathrm{Fe})$ & $\mathrm{Mn}$ & Djotsk \\
\hline $\mathrm{N}$ & 46 & 18 & Margoz & Fe skarn & $\mathrm{Fe}$ & Kizir-Kazyr \\
\hline $\mathrm{N}$ & 46 & 19 & Rudny Kaskad & Fe skarn & $\mathrm{Fe}$ & Kizir-Kazyr \\
\hline $\mathrm{N}$ & 46 & 2 & Leiba & Polymetallic $(\mathrm{Pb}, \mathrm{Zn}, \mathrm{Ag})$ carbonate-hosted metasomatite & $\mathrm{Pb}, \mathrm{Zn}$ & Unassigned \\
\hline $\mathrm{N}$ & 46 & 20 & Terekhovskoye & Fe skarn & $\mathrm{Fe}$ & Kizir-Kazyr \\
\hline $\mathrm{N}$ & 46 & 21 & Odinochnoye & Fe skarn & $\mathrm{Fe}$ & Kizir-Kazyr \\
\hline $\mathrm{N}$ & 46 & 22 & Sydinskoye & Banded iron formation (BIF, Superior Fe) & $\mathrm{Fe}$ & Kizir-Kazyr \\
\hline $\mathrm{N}$ & 46 & 23 & Mulginskoye & Fe skarn & $\mathrm{Fe}$ & Kizir-Kazyr \\
\hline
\end{tabular}




\begin{tabular}{|c|c|c|c|c|c|c|}
\hline Row & Col. & No. & Deposit Name & Mineral Deposit Type & Major Metals & Metallogenic Belt \\
\hline $\mathrm{N}$ & 46 & 24 & Raduga & W-Mo-Be greisen, stockwork, and quartz vein & $\mathrm{Be}$ & Kizhi-Khem \\
\hline $\mathrm{N}$ & 46 & 25 & Burlukskoye & Fe skarn & $\mathrm{Fe}$ & Kizir-Kazyr \\
\hline $\mathrm{N}$ & 46 & 26 & Samson & Fe skarn & $\mathrm{Fe}$ & Kiyalykh-Uzen \\
\hline $\mathrm{N}$ & 46 & 27 & Tibik & Ag-Sb vein & $\mathrm{Sb}$ & Sorsk \\
\hline $\mathrm{N}$ & 46 & 28 & Petropavlovskoye & Fe skarn & $\mathrm{Fe}$ & Kizir-Kazyr \\
\hline $\mathrm{N}$ & 46 & 29 & Okunevskoye & W-Mo-Be greisen, stockwork, and quartz vein & $\mathrm{Be}, \mathrm{CaF} 2$ & Kizhi-Khem \\
\hline $\mathrm{N}$ & 46 & 3 & Seibinskoye 2 & Sedimentary phosphate & Phosphorite & Bellyk \\
\hline $\mathrm{N}$ & 46 & 30 & Telekskoye & Weathering crust and karst phosphate & Phosphorite & Bellyk \\
\hline $\mathrm{N}$ & 46 & 31 & Znamenskoye & Fe skarn & $\mathrm{Fe}$ & Kizir-Kazyr \\
\hline $\mathrm{N}$ & 46 & 32 & Tayatskoye & Fe skarn & $\mathrm{Fe}$ & Kizir-Kazyr \\
\hline $\mathrm{N}$ & 46 & 33 & Tabratskoye & Fe skarn & $\mathrm{Fe}$ & Kizir-Kazyr \\
\hline $\mathrm{N}$ & 46 & 34 & Khabalykskoye & Fe skarn & $\mathrm{Fe}$ & Kizir-Kazyr \\
\hline $\mathrm{N}$ & 46 & 35 & Oktyabrskoye 1 & Granitoid-related $\mathrm{Au}$ vein & $\mathrm{Au}$ & Kizhi-Khem \\
\hline $\mathrm{N}$ & 46 & 36 & Martyuhinskoye & Bedded barite & Barite & Bellyk \\
\hline $\mathrm{N}$ & 46 & 37 & Oktyabrskoye 2 & Clastic sediment-hosted $\mathrm{Hg} \pm \mathrm{Sb}$ & $\mathrm{Hg}$ & Sistigkhem \\
\hline $\mathrm{N}$ & 46 & 38 & Izykhskoye & Fe skarn & $\mathrm{Fe}$ & Kizir-Kazyr \\
\hline $\mathrm{N}$ & 46 & 38 & Poselschik & $\mathrm{Cu}( \pm \mathrm{Fe}, \mathrm{Au}, \mathrm{Ag}, \mathrm{Mo})$ skarn & $\mathrm{Cu}, \mathrm{Mo}$ & Kiyalykh-Uzen \\
\hline $\mathrm{N}$ & 46 & 39 & Kukshinskoye & Clastic sediment-hosted $\mathrm{Hg} \pm \mathrm{Sb}$ & $\mathrm{Hg}$ & Sistigkhem \\
\hline $\mathrm{N}$ & 46 & 4 & Belokitatskoye & Volcanogenic-sedimentary $\mathrm{Fe}$ & $\mathrm{Fe}$ & Kizir-Kazyr \\
\hline $\mathrm{N}$ & 46 & 40 & Tolcheinskoye & Bedded barite & $\mathrm{Ba}$ & Bellyk \\
\hline $\mathrm{N}$ & 46 & 41 & Julia Mednaya & $\mathrm{Cu}( \pm \mathrm{Fe}, \mathrm{Au}, \mathrm{Ag}, \mathrm{Mo})$ skarn & $\mathrm{Cu}, \mathrm{Mo}$ & Kiyalykh-Uzen \\
\hline $\mathrm{N}$ & 46 & 42 & Julia Svintsovaya & $\mathrm{Zn}-\mathrm{Pb}(\mathrm{Ag}, \mathrm{Cu}, \mathrm{W})$ skarn & $\mathrm{Pb}$ & Sorsk \\
\hline $\mathrm{N}$ & 46 & 43 & Kapchalskoye & Barite vein & Barite & Chapsordag \\
\hline $\mathrm{N}$ & 46 & 44 & Kysyl-Tashskoye & $\begin{array}{l}\text { Volcanogenic } \mathrm{Zn}-\mathrm{Pb}-\mathrm{Cu} \text { massive sulfide (Kuroko, Altai } \\
\text { type) }\end{array}$ & $\mathrm{Zn}, \mathrm{Pb}, \mathrm{Cu}$ & Ulugoisk \\
\hline $\mathrm{N}$ & 46 & 45 & Sorskoye & Porphyry Mo $( \pm \mathrm{W}, \mathrm{Sn}, \mathrm{Bi})$ & $\mathrm{Mo}, \mathrm{Cu}$ & Sorsk \\
\hline $\mathrm{N}$ & 46 & 46 & Sorminskoye & Bedded barite & Barite & Bellyk \\
\hline $\mathrm{N}$ & 46 & 47 & Kyzyl-Tashtygskoye & $\begin{array}{l}\text { Volcanogenic } \mathrm{Zn}-\mathrm{Pb}-\mathrm{Cu} \text { massive sulfide (Kuroko, Altai } \\
\text { type) }\end{array}$ & $\mathrm{Zn}, \mathrm{Pb}, \mathrm{Cu}$ & Ulugoisk \\
\hline $\mathrm{N}$ & 46 & 48 & Sayanskoye & Serpentinite-hosted asbestos & $\begin{array}{l}\text { Chrysotile } \\
\text { asbestos }\end{array}$ & Khemchik-Kurtushubinsk \\
\hline $\mathrm{N}$ & 46 & 49 & Beiskoye & Porphyry Mo $( \pm \mathrm{W}, \mathrm{Sn}, \mathrm{Bi})$ & Mo & Sorsk \\
\hline $\mathrm{N}$ & 46 & 5 & Alga & Polymetallic $(\mathrm{Pb}, \mathrm{Zn}, \mathrm{Ag})$ carbonate-hosted metasomatite & $\mathrm{Pb}, \mathrm{Zn}$ & Unassigned \\
\hline $\mathrm{N}$ & 46 & 50 & Kyzyk-Chadrskoye & Porphyry Cu-Mo $( \pm \mathrm{Au}, \mathrm{Ag})$ & $\mathrm{Cu}, \mathrm{Mo}$ & Unassigned \\
\hline $\mathrm{N}$ & 46 & 51 & Malo-Shushenskoye & Granitoid-related $\mathrm{Au}$ vein & $\mathrm{Au}$ & Kizir-Kazyr \\
\hline $\mathrm{N}$ & 46 & 52 & Temir-Dag & $\mathrm{Cu}( \pm \mathrm{Fe}, \mathrm{Au}, \mathrm{Ag}, \mathrm{Mo})$ skarn & $\mathrm{Cu}, \mathrm{Mo}$ & Kiyalykh-Uzen \\
\hline $\mathrm{N}$ & 46 & 53 & Taptan-Turazy & Barite vein & Barite & Chapsordag \\
\hline $\mathrm{N}$ & 46 & 54 & Mainskoye & Cyprus $\mathrm{Cu}-\mathrm{Zn}$ massive sulfide & $\mathrm{Cu}$ & North-Sayanian \\
\hline $\mathrm{N}$ & 46 & 55 & Kamyshtinskoye & Porphyry Mo $( \pm \mathrm{W}, \mathrm{Sn}, \mathrm{Bi})$ & Mo & Sorsk \\
\hline $\mathrm{N}$ & 46 & 56 & Igr-Golskoye & Polymetallic $(\mathrm{Pb}, \mathrm{Zn}, \mathrm{Ag})$ carbonate-hosted metasomatite & $\mathrm{Pb}, \mathrm{Zn}$ & Sorsk \\
\hline $\mathrm{N}$ & 46 & 57 & Bazikskoye & Polymetallic $(\mathrm{Pb}, \mathrm{Zn}, \mathrm{Ag})$ carbonate-hosted metasomatite & $\mathrm{Cu}$ & Chapsordag \\
\hline $\mathrm{N}$ & 46 & 58 & Chapsordag & Barite vein & Barite & Chapsordag \\
\hline $\mathrm{N}$ & 46 & 59 & Obkolskoye & W-Mo-Be greisen, stockwork, and quartz vein & $\mathrm{Be}$ & Kizhi-Khem \\
\hline $\mathrm{N}$ & 46 & 6 & Zhurskoye & Carbonate-hosted fluorspar & Fluorite & Chapsordag \\
\hline $\mathrm{N}$ & 46 & 60 & Azkizskoye & Rhodusite asbestos & Asbestos & Unassigned \\
\hline $\mathrm{N}$ & 46 & 61 & Butrakhtinskoye & $\mathrm{Ni}-\mathrm{Co}$ arsenide vein & $\mathrm{Co}, \mathrm{Cu}$ & Kharadzhulsk \\
\hline $\mathrm{N}$ & 46 & 62 & Abakanskoye & Fe skarn & $\mathrm{Fe}$ & North-Sayanian \\
\hline $\mathrm{N}$ & 46 & 63 & Karbai & Fe skarn & $\mathrm{Fe}$ & Kizir-Kazyr \\
\hline $\mathrm{N}$ & 46 & 64 & Sitikskoye & Volcanic-hosted Hg & $\mathrm{Hg}$ & Unassigned \\
\hline $\mathrm{N}$ & 46 & 7 & Konstantinovskoye & Granitoid-related $\mathrm{Au}$ vein & $\mathrm{Au}$ & Kizir-Kazyr \\
\hline $\mathrm{N}$ & 46 & 8 & Olkhovskoye & Granitoid-related $\mathrm{Au}$ vein & $\mathrm{Au}$ & Kizir-Kazyr \\
\hline
\end{tabular}




\begin{tabular}{|c|c|c|c|c|c|c|}
\hline Row & Col. & No. & Deposit Name & Mineral Deposit Type & Major Metals & Metallogenic Belt \\
\hline $\mathrm{N}$ & 46 & 9 & Medvezhie & Granitoid-related $\mathrm{Au}$ vein & $\mathrm{Au}$ & Kizir-Kazyr \\
\hline $\mathrm{N}$ & 47 & 1 & Beloziminskoye & REE $( \pm \mathrm{Ta}, \mathrm{Nb}, \mathrm{Fe})$ carbonatite & $\mathrm{Nb}, \mathrm{Ta}$ & Prisayanskiy \\
\hline $\mathrm{N}$ & 47 & 10 & Zashikhinskoe & Ta-Nb-REE alkaline metasomatite & $\mathrm{Ta}, \mathrm{Nb}$ & Zashikhinskiy \\
\hline $\mathrm{N}$ & 47 & 11 & Verkhne-Iiskoye & Mafic-ultramafic related Ti-Fe (V) & $\mathrm{Ti}, \mathrm{Fe}$ & Iiskiy \\
\hline $\mathrm{N}$ & 47 & 12 & Botogolskoe & Magmatic nepheline & $\mathrm{Al}$ & Bokson-Kitoiskiy \\
\hline $\mathrm{N}$ & 47 & 13 & Zun-Kholba & $\mathrm{Au}$ in shear zone and quartz vein & $\mathrm{Au}$ & Bokson-Kitoiskiy \\
\hline $\mathrm{N}$ & 47 & 14 & Barun-Kholba & $\mathrm{Au}$ in shear zone and quartz vein & $\mathrm{Au}$ & Bokson-Kitoiskiy \\
\hline $\mathrm{N}$ & 47 & 15 & Pionerskoye 2 & $\mathrm{Au}$ in shear zone and quartz vein & $\mathrm{Au}$ & Bokson-Kitoiskiy \\
\hline $\mathrm{N}$ & 47 & 16 & Ilchirskoye & Serpentinite-hosted asbestos & $\begin{array}{l}\text { Chrysotile } \\
\text { asbestos }\end{array}$ & Bokson-Kitoiskiy \\
\hline $\mathrm{N}$ & 47 & 17 & Aksug & Porphyry Cu-Mo $( \pm \mathrm{Au}, \mathrm{Ag})$ & $\mathrm{Cu}, \mathrm{Mo}$ & Kizhi-Khem \\
\hline $\mathrm{N}$ & 47 & 18 & Daschkhemskoye & Porphyry Mo $( \pm \mathrm{W}, \mathrm{Sn}, \mathrm{Bi})$ & Mo & Kizhi-Khem \\
\hline $\mathrm{N}$ & 47 & 19 & Kazyrskoye & W-Mo-Be greisen, stockwork, and quartz vein & $\mathrm{Be}, \mathrm{Li}$ & Kizhi-Khem \\
\hline $\mathrm{N}$ & 47 & 2 & Vishnyakovskoye & REE-Li pegmatite & $\mathrm{Ta}$ & Tagulskiy \\
\hline $\mathrm{N}$ & 47 & 20 & Ulug-Alymskoye & W-Mo-Be greisen, stockwork, and quartz vein & W, Mo & Kizhi-Khem \\
\hline $\mathrm{N}$ & 47 & 21 & Aryskanskoye 1 & Ta-Nb-REE alkaline metasomatite & $\mathrm{REE}, \mathrm{Nb}$, & Kizhi-Khem \\
\hline $\mathrm{N}$ & 47 & 22 & Ulug-Odir-Oiy & Peralkaline granitoid-related Nb-Zr-REE & $\mathrm{Ta}, \mathrm{Nb}, \mathrm{REE}$ & Unassigned \\
\hline $\mathrm{N}$ & 47 & 23 & Dalneye & $\begin{array}{l}\text { Volcanogenic } \mathrm{Zn}-\mathrm{Pb}-\mathrm{Cu} \text { massive sulfide (Kuroko, Altai } \\
\text { type) }\end{array}$ & $\mathrm{Zn}, \mathrm{Pb}, \mathrm{Cu}$ & Ulugoisk \\
\hline $\mathrm{N}$ & 47 & 3 & Malo-Tagulskoye & Mafic-ultramafic related Ti-Fe (V) & $\mathrm{Ti}, \mathrm{Fe}$ & Tagulskiy \\
\hline $\mathrm{N}$ & 47 & 4 & Karasuk & Polymetallic $(\mathrm{Pb}, \mathrm{Zn}, \mathrm{Ag})$ carbonate-hosted metasomatite & $\mathrm{Pb}, \mathrm{Zn}$ & Sorsk \\
\hline $\mathrm{N}$ & 47 & 5 & Gutaro-Biryusinskoye & Muscovite pegmatite & Muscovite & Tagulskiy \\
\hline $\mathrm{N}$ & 47 & 6 & Ingashinskoye & Diamond-bearing kimberlite & Diamond & Prisayanskiy \\
\hline $\mathrm{N}$ & 47 & 7 & Sredneziminskoye & $\mathrm{REE}( \pm \mathrm{Ta}, \mathrm{Nb}, \mathrm{Fe})$ carbonatite & $\mathrm{Ta}, \mathrm{Nb}$ & Prisayanskiy \\
\hline $\mathrm{N}$ & 47 & 8 & Gorkhonskoye & Clastic sediment-hosted $\mathrm{Hg} \pm \mathrm{Sb}$ & $\mathrm{Hg}$ & Zashikhinskiy \\
\hline $\mathrm{N}$ & 47 & 9 & Agulskoye & Porphyry $\mathrm{Cu}-\mathrm{Mo}( \pm \mathrm{Au}, \mathrm{Ag})$ & $\mathrm{Cu}$ & Agulsk \\
\hline $\mathrm{N}$ & 48 & 1 & Sosnovy Baits & Banded iron formation (BIF, Superior Fe) & $\mathrm{Fe}$ & Sharizhalgaiskiy \\
\hline $\mathrm{N}$ & 48 & 2 & Onotskoe & Talc (magnesite) replacement & Talc & Prisayanskiy \\
\hline $\mathrm{N}$ & 48 & 3 & Savinskoe & Talc (magnesite) replacement & Magnesite, talc & Sharizhalgaiskiy \\
\hline $\mathrm{N}$ & 48 & 4 & Zhidoyskoye & Mafic-ultramafic related Ti-Fe (V) & $\mathrm{Ti}, \mathrm{Fe}$ & Prisayanskiy \\
\hline $\mathrm{N}$ & 49 & 1 & Ulzutuiskoye & $\begin{array}{l}\text { Volcanogenic-hydrothermal-sedimentary massive sulfide } \\
\mathrm{Pb}-\mathrm{Zn}( \pm \mathrm{Cu})\end{array}$ & $\mathrm{Zn}$ & Ozerninskiy \\
\hline $\mathrm{N}$ & 49 & 2 & Ozernoye 2 & $\begin{array}{l}\text { Volcanogenic-hydrothermal-sedimentary massive sulfide } \\
\mathrm{Pb}-\mathrm{Zn}( \pm \mathrm{Cu})\end{array}$ & $\mathrm{Zn}$ & Ozerninskiy \\
\hline $\mathrm{N}$ & 49 & 3 & Gundui & Sediment-hosted $\mathrm{Cu}$ & $\mathrm{Cu}$ & Ozerninsky \\
\hline $\mathrm{N}$ & 49 & 4 & Kydzhimitskoye & Cassiterite-sulfide-silicate vein and stockwork & Sn & Eravninsky \\
\hline $\mathrm{N}$ & 49 & 5 & Egitinskoye & Carbonate-hosted fluorspar & Fluorite & Eravninsky \\
\hline $\mathrm{N}$ & 49 & 6 & Lugovoye & Polymetallic $(\mathrm{Pb}, \mathrm{Zn}, \mathrm{Ag})$ carbonate-hosted metasomatite & $\mathrm{Pb}$ & Pribaikalskiy \\
\hline $\mathrm{N}$ & 50 & 1 & Mokhovoye & Porphyry Sn & $\mathrm{Sn}$ & Muiskiy \\
\hline $\mathrm{N}$ & 50 & 10 & Budyumkanskoye & Sn-W greisen, stockwork, and quartz vein & Sn & East Mongolian-Priargunskiy \\
\hline $\mathrm{N}$ & 50 & 11 & Solonechinskoe & Carbonate-hosted $\mathrm{Hg}-\mathrm{Sb}$ & $\mathrm{Sb}$ & East Mongolian-Priargunskiy \\
\hline $\mathrm{N}$ & 50 & 12 & Kariiyskoye & Granitoid-related $\mathrm{Au}$ vein & $\mathrm{Au}$ & Shilkinsko-Tukuringrskiy \\
\hline $\mathrm{N}$ & 50 & 13 & Yekaterininskoye & Polymetallic $(\mathrm{Pb}, \mathrm{Zn}, \mathrm{Ag})$ carbonate-hosted metasomatite & $\mathrm{Pb}$ & East Mongolian-Priarguskiy \\
\hline $\mathrm{N}$ & 50 & 14 & Zhirekenskoye & Porphyry Mo ( \pm W, Sn, Bi) & Mo & Shilkinsko-Tukuringrskiy \\
\hline $\mathrm{N}$ & 50 & 15 & Usuglinskoye & Fluorspar vein & Fluorite & Nerchinsko-Dzheltulakskiy \\
\hline $\mathrm{N}$ & 50 & 16 & Darasunskoye & Granitoid-related $\mathrm{Au}$ vein & $\mathrm{Au}$ & Nerchinskiy \\
\hline $\mathrm{N}$ & 50 & 17 & Teremkinskoye & Granitoid-related Au vein & $\mathrm{Au}$ & Nerchinsko-Dzheltulakskiy \\
\hline $\mathrm{N}$ & 50 & 18 & Talatuiskoye & Granitoid-related Au vein & $\mathrm{Au}$ & Nerchinsko-Dzheltulakskiy \\
\hline $\mathrm{N}$ & 50 & 19 & Kruchininskoye & Mafic-ultramafic related Ti-Fe (V) & $\mathrm{Ti}$ & Kruchininskiy \\
\hline $\mathrm{N}$ & 50 & 2 & Irokinda & Au in shear zone and quartz vein & $\mathrm{Au}$ & Muiskiy \\
\hline $\mathrm{N}$ & 50 & 3 & Muoklakanskoye & W-Mo-Be greisen, stockwork, and quartz vein & $\mathrm{W}$ & Nerchinskiy \\
\hline
\end{tabular}




\begin{tabular}{|c|c|c|c|c|c|c|}
\hline $\mathrm{N}$ & 50 & 4 & Ukonikskoe & Granitoid-related Au vein & $\mathrm{Au}$ & Shilkinsko-Tukuringrskiy \\
\hline $\mathrm{N}$ & 50 & 5 & Orekitkanskoye & Porphyry Mo $( \pm \mathrm{W}, \mathrm{Sn}, \mathrm{Bi})$ & Mo & Karengskiy \\
\hline $\mathrm{N}$ & 50 & 6 & Itakinskoye & Granitoid-related Au vein & $\mathrm{Au}$ & Shilkinsko-Tukuringrskiy \\
\hline $\mathrm{N}$ & 50 & 7 & Davenda & Porphyry Mo $( \pm \mathrm{W}, \mathrm{Sn}, \mathrm{Bi})$ & Mo & Shilkinsko-Tukuringrskiy \\
\hline $\mathrm{N}$ & 50 & 8 & Klyuchevskoye & Granitoid-related $\mathrm{Au}$ vein & $\mathrm{Au}$ & Shilkinsko-Tukuringrskiy \\
\hline $\mathrm{N}$ & 50 & 9 & Aleksandrovskoye & Granitoid-related $\mathrm{Au}$ vein & $\mathrm{Au}$ & Shilkinsko-Takuringrskiy \\
\hline $\mathrm{N}$ & 51 & 1 & Kavakta & Mafic-ultramafic related Ti-Fe (V) & $\mathrm{P}, \mathrm{Ti}, \mathrm{Fe}$ & Kavakta \\
\hline $\mathrm{N}$ & 51 & 2 & Bamskoe (Chul'bango) & Au-Ag epithermal vein & $\mathrm{Au}, \mathrm{Ag}$ & North Stanovoy \\
\hline $\mathrm{N}$ & 51 & 3 & Kirovskoe & Granitoid-related $\mathrm{Au}$ vein & $\mathrm{Au}$ & Shilkinsko-Tukutingrskiy \\
\hline $\mathrm{N}$ & 51 & 4 & Burindinskoe & Au-Ag epithermal vein & $\mathrm{Au}, \mathrm{Ag}$ & North Stanovoy \\
\hline $\mathrm{N}$ & 51 & 5 & Berezitovoe & Polymetallic $\mathrm{Pb}-\mathrm{Zn} \pm \mathrm{Cu}( \pm \mathrm{Ag}, \mathrm{Au})$ vein and stockwork & $\mathrm{Zn}, \mathrm{Pb}, \mathrm{Au}, \mathrm{Ag}$ & Shilkinsko-Tukutingrskiy \\
\hline $\mathrm{N}$ & 51 & 6 & $\begin{array}{l}\text { Ershiyizhan, Heilongjiang } \\
\text { Province }\end{array}$ & Porphyry $\mathrm{Cu}( \pm \mathrm{Au})$ & $\mathrm{Au}$ & East Mongolian-Priargunskiy- Deerbuga \\
\hline $\mathrm{N}$ & 52 & 1 & Borgulican & Porphyry $\mathrm{Cu}( \pm \mathrm{Au})$ & $\mathrm{Cu}, \mathrm{Au}$ & North Bureya \\
\hline $\mathrm{N}$ & 52 & 1 & Kolchedanny Utyos & Volcanic-hosted Au-base-metal metasomatite & $\mathrm{Au}$ & Tyrkanda-Stanovoy \\
\hline $\mathrm{N}$ & 52 & 2 & Malomyr & $\mathrm{Au}$ in shear zone and quartz vein & $\mathrm{Au}$ & Kerbi-Selemdzha \\
\hline $\mathrm{N}$ & 52 & 3 & Zolotaya Gora & $\mathrm{Au}$ in shear zone and quartz vein & $\mathrm{Au}$ & Djeltulaksky \\
\hline $\mathrm{N}$ & 52 & 4 & Kamenushinskoe & $\begin{array}{l}\text { Volcanogenic } \mathrm{Zn}-\mathrm{Pb}-\mathrm{Cu} \text { massive sulfide (Kuroko, Altai } \\
\text { type) }\end{array}$ & $\mathrm{Cu}, \mathrm{FeS}$ & Shimanovsk-Gar \\
\hline $\mathrm{N}$ & 52 & 5 & Pioneer & Granitoid-related $\mathrm{Au}$ vein & $\mathrm{Au}$ & North Bureya \\
\hline $\mathrm{N}$ & 52 & 6 & Gar & Volcanogenic-sedimentary Fe & $\mathrm{Fe}$ & Shimanovsk-Gar \\
\hline $\mathrm{N}$ & 52 & 7 & Pokrovskoe & Au-Ag epithermal vein & $\mathrm{Au}, \mathrm{Ag}$ & North Bureya \\
\hline $\mathrm{N}$ & 52 & 8 & Chagoyan & Sedimentary exhalative Pb-Zn (SEDEX) & $\mathrm{Pb}, \mathrm{Zn}, \mathrm{Ag}$ & Chagoyan \\
\hline $\mathrm{N}$ & 53 & 1 & Etara & Granitoid-related Au vein & $\mathrm{Au}$ & Preddzhugdzhursky \\
\hline $\mathrm{N}$ & 53 & 10 & Nelkanskoe & Sedimentary phosphate & $\mathrm{P}$ & Uda-Shantar \\
\hline $\mathrm{N}$ & 53 & 11 & Ir-Nimiiskoe-2 & Sedimentary phosphate & $\mathrm{P}$ & Uda-Shantar \\
\hline $\mathrm{N}$ & 53 & 12 & Ir-Nimiiskoe-1 & Volcanogenic-sedimentary Mn & $\mathrm{Mn}$ & Uda-Shantar \\
\hline $\mathrm{N}$ & 53 & 13 & Kolchedannyi Utyos & $\mathrm{Au}$ in shear zone and quartz vein & $\mathrm{Au}$ & Tyrkanda-Stanovoy \\
\hline $\mathrm{N}$ & 53 & 14 & Milkanskoe & Volcanogenic-sedimentary Fe & $\mathrm{Fe}$ & Uda-Shantar \\
\hline $\mathrm{N}$ & 53 & 15 & Lagapskoe & Sedimentary phosphate & $\mathrm{P}$ & Uda-Shantar \\
\hline $\mathrm{N}$ & 53 & 16 & Boguchanskoe & W-Mo-Be greisen, stockwork, and quartz vein & $\mathrm{W}$ & Pilda-Limuri \\
\hline $\mathrm{N}$ & 53 & 17 & Galamskoe & Volcanogenic-sedimentary $\mathrm{Fe}$ & $\mathrm{Fe}$ & Uda-Shantar \\
\hline $\mathrm{N}$ & 53 & 18 & Davakit & Anorthosite-apatite Ti-P & Ti, P & Baladek \\
\hline $\mathrm{N}$ & 53 & 19 & Gerbikanskoe & Volcanogenic-sedimentary $\mathrm{Fe}$ & $\mathrm{Fe}$ & Uda-Shantar \\
\hline $\mathrm{N}$ & 53 & 2 & Kuma & $\mathrm{Cu}( \pm \mathrm{Fe}, \mathrm{Au}, \mathrm{Ag}, \mathrm{Mo})$ skarn & $\mathrm{Cu}$ & Preddzhugdzhursky \\
\hline $\mathrm{N}$ & 53 & 20 & Itmatinskoe & Volcanogenic-sedimentary $\mathrm{Fe}$ & $\mathrm{Fe}$ & Uda-Shantar \\
\hline $\mathrm{N}$ & 53 & 21 & Kurumskoe & Volcanogenic-sedimentary Fe & $\mathrm{Fe}$ & Uda-Shantar \\
\hline $\mathrm{N}$ & 53 & 22 & Ingagli & Au in shear zone and quartz vein & $\mathrm{Au}$ & Kerbi-Selemdzha \\
\hline $\mathrm{N}$ & 53 & 23 & Kharga & $\mathrm{Au}$ in shear zone and quartz vein & $\mathrm{Au}$ & Kerbi-Selemdzha \\
\hline $\mathrm{N}$ & 53 & 24 & Lednikovy-Sarmaka & W-Mo-Be greisen, stockwork, and quartz vein & $\mathrm{W}$ & Ezop-Yam-Alin \\
\hline $\mathrm{N}$ & 53 & 25 & Tokur & $\mathrm{Au}$ in shear zone and quartz vein & $\mathrm{Au}$ & Kerbi-Selemdzha \\
\hline $\mathrm{N}$ & 53 & 26 & Afanas'evskoe & $\mathrm{Au}$ in shear zone and quartz vein & $\mathrm{Au}$ & Kerbi - Selemdzha \\
\hline $\mathrm{N}$ & 53 & 27 & Zazubrinskoe & $\mathrm{Au}$ in shear zone and quartz vein & $\mathrm{Au}$ & Kerbi-Selemdzha \\
\hline $\mathrm{N}$ & 53 & 28 & Poiskovoe & Granitoid-related $\mathrm{Au}$ vein & $\mathrm{Au}$ & Kerbi -Selemdzha \\
\hline $\mathrm{N}$ & 53 & 29 & Ezop & Sn-W greisen, stockwork, and quartz vein & Sn & Ezop-Yam-Alin \\
\hline $\mathrm{N}$ & 53 & 3 & North-Shantarskoe & Sedimentary phosphate & $\mathrm{P}$ & Uda-Shantar \\
\hline $\mathrm{N}$ & 53 & 30 & Sagurskoe & $\mathrm{Au}$ in shear zone and quartz vein & $\mathrm{Au}$ & Kerbi -Selemdzha \\
\hline $\mathrm{N}$ & 53 & 31 & Talaminskoe & Cassiterite-sulfide-silicate vein and stockwork & $\mathrm{Sb}, \mathrm{Au}$ & Kerbi-Selemdzha \\
\hline $\mathrm{N}$ & 53 & 32 & Olgakanskoe & Sn-W greisen, stockwork, and quartz vein & $\mathrm{Sn}$ & Ezop-Yam-Alin \\
\hline $\mathrm{N}$ & 53 & 4 & Feklistov & Zoned mafic-ultramafic Cr-PGE & PGE & Kondyor-Feklistov \\
\hline $\mathrm{N}$ & 53 & 5 & Mayskoye 1 & Carbonate-hosted Pb-Zn (Mississippi valley type) & $\mathrm{Zn}, \mathrm{Pb}$ & Preddzhugdzhurskiy \\
\hline
\end{tabular}




\begin{tabular}{|c|c|c|c|c|c|c|}
\hline Row & Col. & No. & Deposit Name & Mineral Deposit Type & Major Metals & Metallogenic Belt \\
\hline $\mathrm{N}$ & 53 & 6 & Gayumskoe & Anorthosite-apatite Ti-P & $\mathrm{Ti}, \mathrm{P}$ & Baladek \\
\hline $\mathrm{N}$ & 53 & 7 & Maimakanskoe & Anorthosite-apatite Ti-P & Ti, P & Baladek \\
\hline $\mathrm{N}$ & 53 & 8 & Dzhaninskoe & Anorthosite-apatite Ti-P & Ti, P & Baladek \\
\hline $\mathrm{N}$ & 53 & 9 & Bogidenskoe & Anorthosite apatite Ti-P & $\mathrm{Ti}, \mathrm{P}$ & Baladek \\
\hline $\mathrm{N}$ & 54 & 1 & Mnogovershinnoe & $\mathrm{Au}-\mathrm{Ag}$ epithermal vein & $\mathrm{Au}, \mathrm{Ag}$ & Lower Amur \\
\hline $\mathrm{N}$ & 54 & 2 & Iskinskoe (Askum) & Epithermal quartz-alunite & $\mathrm{Al}$ & Lower Amur \\
\hline $\mathrm{N}$ & 54 & 3 & Bichinskoe & Sn-W greisen, stockwork, and quartz vein & $\mathrm{W}, \mathrm{Sn}$ & Lower Amur \\
\hline $\mathrm{O}$ & 44 & 1 & Kolpashevskoye & Sedimentary Fe-V & $\mathrm{Fe}$ & Bakcharsk \\
\hline $\mathrm{O}$ & 44 & 2 & Parabel-Chuzikskoye & Sedimentary siderite $\mathrm{Fe}$ & $\mathrm{Fe}$ & Bakcharsk \\
\hline $\mathrm{O}$ & 44 & 3 & Bakcharskoye & Banded iron formation (BIF, Superior Fe) & $\mathrm{Fe}$ & Bakcharsk \\
\hline $\mathrm{O}$ & 44 & 4 & Parbigskoye & Sedimentary siderite $\mathrm{Fe}$ & $\mathrm{Fe}$ & Bakcharsk \\
\hline $\mathrm{O}$ & 45 & 1 & Iverskoye & Sedimentary siderite $\mathrm{Fe}$ & $\mathrm{Fe}$ & Unassigned \\
\hline $\mathrm{O}$ & 45 & 2 & Semiluzhinskoye & Clastic-sediment-hosted $\mathrm{Sb}-\mathrm{Au}$ & $\mathrm{Sb}, \mathrm{Au}$ & Unassigned \\
\hline $\mathrm{O}$ & 46 & 1 & Ust-Talskoye & REE-Li pegmatite & $\mathrm{Li}, \mathrm{Sn}$ & Tatarsko-Tyradinskoye \\
\hline $\mathrm{O}$ & 46 & 10 & Mutovskoye & Carbonate-hosted $\mathrm{Hg}$-Sb & $\mathrm{Hg}$ & Unassigned \\
\hline $\mathrm{O}$ & 46 & 11 & Polkan Gora & Fe skarn & $\mathrm{Fe}$ & Vorogovsko-Angarsk \\
\hline $\mathrm{O}$ & 46 & 12 & Lendakhskoye & Fe skarn & $\mathrm{Fe}$ & Vorogovsko-Angarsk \\
\hline $\mathrm{O}$ & 46 & 13 & Enashiminskoye 2 & Fe skarn & $\mathrm{Fe}$ & Vorogovsko-Angarsk \\
\hline $\mathrm{O}$ & 46 & 14 & Ilinskoye & W-Mo-Be greisen, stockwork, and quartz vein & $\mathrm{W}$ & Tatarsko-Tyradinsk \\
\hline $\mathrm{O}$ & 46 & 15 & Nikolaevskoye & Au in shear zone and quartz vein & $\mathrm{Au}$ & Central-Yenisey \\
\hline $\mathrm{O}$ & 46 & 16 & Vasilievskoye & $\mathrm{Au}$ in shear zone and quartz vein & $\mathrm{Au}$ & Central-Yenisey \\
\hline $\mathrm{O}$ & 46 & 17 & Sokhatinoye & Bauxite (karst type) & $\mathrm{Al}$ & Verkhoturovsk \\
\hline $\mathrm{O}$ & 46 & 18 & Gerfedskoye & Au in shear zone and quartz vein & $\mathrm{Au}$ & Central-Yenisey \\
\hline $\mathrm{O}$ & 46 & 19 & Mokrinskoye & Sedimentary Fe-V & $\mathrm{Fe}$ & Angara-Pit \\
\hline $\mathrm{O}$ & 46 & 2 & Enashiminskoye 1 & Clastic-sediment-hosted Sb-Au & $\mathrm{Au}$ & Central-Yenisey \\
\hline $\mathrm{O}$ & 46 & 20 & Verchne-Kamenskoye & Polymetallic $\mathrm{Pb}-\mathrm{Zn} \pm \mathrm{Cu}( \pm \mathrm{Ag}, \mathrm{Au})$ vein and stockwork & $\mathrm{Pb}, \mathrm{Zn}$ & Vorogovsko-Angarsk \\
\hline $\mathrm{O}$ & 46 & 21 & Verkhoturovskoye & Bauxite (karst type) & $\mathrm{Al}$ & Verkhoturovsk \\
\hline $\mathrm{O}$ & 46 & 22 & Kirgiteiskoye 2 & Talc (magnesite) replacement & Talc & Verkhoturovsk \\
\hline $\mathrm{O}$ & 46 & 23 & Mulinskoye & REE-Li pegmatite & $\mathrm{Li}$ & Tatarsko-Tyradinsk \\
\hline $\mathrm{O}$ & 46 & 24 & Kirgiteiskoye 1 & Bauxite (karst type) & $\mathrm{Al}$ & Verkhoturovsk \\
\hline $\mathrm{O}$ & 46 & 25 & Murlinoye & Bauxite (karst type) & $\mathrm{Al}$ & Verkhoturovsk \\
\hline $\mathrm{O}$ & 46 & 26 & Sredne-Tatarskoye & Bauxite (karst type) & Al & Verkhoturovsk \\
\hline $\mathrm{O}$ & 46 & 27 & Teneginskoye & Carbonate-hosted Pb-Zn (Mississippi valley type) & $\mathrm{Zn}, \mathrm{Pb}$ & Vorogovsko-Angarsk \\
\hline $\mathrm{O}$ & 46 & 28 & Udorongovskoye & Volcanogenic-sedimentary Fe & $\mathrm{Fe}$ & Angara-Pit \\
\hline $\mathrm{O}$ & 46 & 29 & Udereiskoye & Clastic-sediment-hosted Sb-Au & $\mathrm{Sb}, \mathrm{Au}$ & Central-Yenisey \\
\hline $\mathrm{O}$ & 46 & 3 & Oleniya Gora & W-Mo-Be greisen, stockwork, and quartz vein & $\mathrm{W}$ & Tatarsko-Tyradinsk \\
\hline $\mathrm{O}$ & 46 & 30 & Detalnoye & Carbonate-hosted $\mathrm{Hg}-\mathrm{Sb}$ & $\mathrm{Hg}$ & Unassigned \\
\hline $\mathrm{O}$ & 46 & 31 & Nizhne-Angarskoye & Sedimentary siderite $\mathrm{Fe}$ & $\mathrm{Fe}$ & Angara-Pit \\
\hline $\mathrm{O}$ & 46 & 32 & Goltsovoye & W-Mo-Be greisen, stockwork, and quartz vein & $\mathrm{W}, \mathrm{Sn}$ & Tatarsko-Tyradinsk \\
\hline $\mathrm{O}$ & 46 & 33 & Rudakovskoye & Carbonate-hosted $\mathrm{Pb}-\mathrm{Zn}$ (Mississippi valley type) & $\mathrm{Pb}, \mathrm{Zn}$ & Vorogovsko-Angarsk \\
\hline $\mathrm{O}$ & 46 & 34 & Moryanikhinskoye & Carbonate-hosted Pb-Zn (Mississippi valley type) & $\mathrm{Pb}, \mathrm{Zn}$ & Vorogovsko-Angarsk \\
\hline $\mathrm{O}$ & 46 & 35 & Razdolninskoye & Clastic-sediment-hosted Sb-Au & $\mathrm{Sb}$ & Central-Yenisey \\
\hline $\mathrm{O}$ & 46 & 36 & Lineinoye & Sedimentary exhalative Pb-Zn (SEDEX) & $\mathrm{Zn}, \mathrm{Pb}$ & Vorogovsko-Angarsk \\
\hline $\mathrm{O}$ & 46 & 37 & Dolgozhdannoye & Sedimentary bauxite & $\mathrm{Al}$ & Verkhoturovsk \\
\hline $\mathrm{O}$ & 46 & 38 & Kiiskoye & Weathering crust carbonatite REE-Zr-Nb-Li & $\begin{array}{l}\mathrm{REE}, \mathrm{Zr}, \mathrm{Nb}, \\
\mathrm{Li}\end{array}$ & Tatarsko-Tyradinsk \\
\hline $\mathrm{O}$ & 46 & 39 & Tatarskoye & $\mathrm{REE}( \pm \mathrm{Ta}, \mathrm{Nb}, \mathrm{Fe})$ carbonatite & REE, $\mathrm{Ta}, \mathrm{Nb}$ & Tatarsko-Tyradinsk \\
\hline $\mathrm{O}$ & 46 & 4 & Visokaya Gora & W-Mo-Be greisen, stockwork, and quartz vein & $\mathrm{W}, \mathrm{Mo}, \mathrm{Au}, \mathrm{Sb}$ & Tatarsko-Tyradinsk \\
\hline $\mathrm{O}$ & 46 & 40 & Krutoye & Sedimentary exhalative Pb-Zn (SEDEX) & $\mathrm{Zn}, \mathrm{Pb}$ & Vorogovsko-Angarsk \\
\hline $\mathrm{O}$ & 46 & 41 & Kondakovskoye & REE-Li pegmatite & $\mathrm{Be}$ & Tatarsko-Tyradinsk \\
\hline
\end{tabular}




\begin{tabular}{|c|c|c|c|c|c|c|}
\hline Row & Col. & No. & Deposit Name & Mineral Deposit Type & Major Metals & Metallogenic Belt \\
\hline $\mathrm{O}$ & 46 & 42 & Gorevskoye & Sedimentary exhalative $\mathrm{Pb}-\mathrm{Zn}$ (SEDEX) & $\mathrm{Pb}, \mathrm{Zn}$ & Vorogovsko-Angarsk \\
\hline $\mathrm{O}$ & 46 & 43 & Tchernoretchenskoye & Clastic sediment-hosted $\mathrm{Hg} \pm \mathrm{Sb}$ & $\mathrm{Hg}$ & Unassigned \\
\hline $\mathrm{O}$ & 46 & 44 & Ust-Angarskoye & Carbonate-hosted $\mathrm{Pb}-\mathrm{Zn}$ (Mississippi valley type) & $\mathrm{Pb}, \mathrm{Zn}$ & Vorogovsko-Angarsk \\
\hline $\mathrm{O}$ & 46 & 45 & Kurishskoye & Sediment-hosted $\mathrm{Cu}$ & $\mathrm{Cu}$ & Bedobinsk \\
\hline $\mathrm{O}$ & 46 & 46 & Predivinskoye & Banded iron formation (BIF, Superior Fe) & $\mathrm{Fe}$ & Kansk \\
\hline $\mathrm{O}$ & 46 & 47 & Kuzeevskoye & $\mathrm{Au}$ in shear zone and quartz vein & $\mathrm{Au}$ & Kansk \\
\hline $\mathrm{O}$ & 46 & 48 & Bogunai & Au in shear zone and quartz vein & $\mathrm{Au}$ & Kansk \\
\hline $\mathrm{O}$ & 46 & 49 & Barginskoye & REE-Li pegmatite & $\mathrm{Be}$ & Kansk \\
\hline $\mathrm{O}$ & 46 & 5 & Olympiada & $\mathrm{Au}$ in black shale & $\mathrm{Au}$ & Central-Yenisey \\
\hline $\mathrm{O}$ & 46 & 50 & Kanskoye & W-Mo-Be greisen, stockwork, and quartz vein & Mo & Kansk \\
\hline $\mathrm{O}$ & 46 & 51 & Mazulskoye & Volcanogenic-sedimentary Mn & $\mathrm{Mn}$ & Unassigned \\
\hline $\mathrm{O}$ & 46 & 6 & Pravoberezhnoye & Clastic sediment-hosted $\mathrm{Hg} \pm \mathrm{Sb}$ & $\mathrm{Hg}$ & Unassigned \\
\hline $\mathrm{O}$ & 46 & 7 & Ayakhta & $\mathrm{Au}$ in shear zone and quartz vein & $\mathrm{Au}$ & Central-Yenisey \\
\hline $\mathrm{O}$ & 46 & 8 & Bolshepitskoye & Polymetallic $(\mathrm{Pb}, \mathrm{Zn}, \mathrm{Ag})$ carbonate-hosted metasomatite & $\mathrm{Pb}, \mathrm{Zn}$ & Vorogovsko-Angarsk \\
\hline $\mathrm{O}$ & 46 & 9 & Ishimbinskoye & Sedimentary siderite $\mathrm{Fe}$ & $\mathrm{Fe}$ & Angara-Pit \\
\hline $\mathrm{O}$ & 47 & 1 & Nizhne-Gondinskoye & Sediment-hosted $\mathrm{Cu}$ & $\mathrm{Cu}$ & Bedobinsk \\
\hline $\mathrm{O}$ & 47 & 10 & Kliminskoye & Fe-Zn skarn & $\mathrm{Fe}$ & Angara-Ilim \\
\hline $\mathrm{O}$ & 47 & 11 & Levoberezhnoye & Fe skarn & $\mathrm{Fe}$ & Angara-Ilim \\
\hline $\mathrm{O}$ & 47 & 12 & Bedobinskoye & Sediment-hosted $\mathrm{Cu}$ & $\mathrm{Cu}$ & Bedobinsk \\
\hline $\mathrm{O}$ & 47 & 13 & Porozhinskoye 2 & Bauxite (karst type) & $\mathrm{Al}$ & Verkhoturovsk \\
\hline $\mathrm{O}$ & 47 & 14 & Kichetskoye & Fe skarn & $\mathrm{Fe}$ & Angara-Ilim \\
\hline $\mathrm{O}$ & 47 & 2 & Chuktukonskoye & $\mathrm{REE}( \pm \mathrm{Ta}, \mathrm{Nb}, \mathrm{Fe})$ carbonatite & $\mathrm{Nb}, \mathrm{REE}$ & Angara-Ilim \\
\hline $\mathrm{O}$ & 47 & 3 & Taloye 1 & Fe skarn & $\mathrm{Fe}$ & Angara-Ilim \\
\hline $\mathrm{O}$ & 47 & 4 & Verkhne-Ollonokonskoye & Fe skarn & $\mathrm{Fe}$ & Angara-Ilim \\
\hline $\mathrm{O}$ & 47 & 5 & Beryambinskoye & Fe-Zn skarn & $\mathrm{Fe}$ & Angara-Ilim \\
\hline $\mathrm{O}$ & 47 & 6 & Pikhtovoye & Fe skarn & $\mathrm{Fe}$ & Angara-Ilim \\
\hline $\mathrm{O}$ & 47 & 7 & Ognenskoye & Fe skarn & $\mathrm{Fe}$ & Angara-Ilim \\
\hline $\mathrm{O}$ & 47 & 8 & Vostok & Fe skarn & $\mathrm{Fe}$ & Angara-Ilim \\
\hline $\mathrm{O}$ & 47 & 9 & Tagarskoye & Fe skarn & $\mathrm{Fe}$ & Angara-Ilim \\
\hline $\mathrm{O}$ & 48 & 1 & Tatyaninskoye & Trap-related Fe skarn (Angara-Ilim type) & $\mathrm{Fe}$ & Angaro-Ilimskiy \\
\hline $\mathrm{O}$ & 48 & 2 & Yubileinoye 1 & Fe skarn & $\mathrm{Fe}$ & Angara-Ilim \\
\hline $\mathrm{O}$ & 48 & 3 & Sputnik 1 & Fe skarn & $\mathrm{Fe}$ & Angara-Ilim \\
\hline $\mathrm{O}$ & 48 & 4 & Atavinskoye 1 & Fe skarn & $\mathrm{Fe}$ & Angara-Ilim \\
\hline $\mathrm{O}$ & 48 & 5 & Nerjundinskoye & Trap-related Fe skarn (Angara-Ilim type) & $\mathrm{Fe}$ & Angaro-Ilimskiy \\
\hline $\mathrm{O}$ & 48 & 6 & Kapaevskoye & Trap-related Fe skarn (Angara-Ilim type) & $\mathrm{Fe}$ & Angaro-Ilimskiy \\
\hline $\mathrm{O}$ & 48 & 7 & Rudnogorskoe & Trap-related Fe skarn (Angara-Ilim type) & $\mathrm{Fe}$ & Angaro-Ilimskiy \\
\hline $\mathrm{O}$ & 48 & 8 & Korshunovskoe & Trap-related Fe skarn (Angara-Ilim type) & $\mathrm{Fe}$ & Angaro-Ilimskiy \\
\hline $\mathrm{O}$ & 48 & 9 & Ponomarjovskoye & Trap-related Fe skarn (Angara-Ilim type) & $\mathrm{Fe}$ & Angaro-Ilimskiy \\
\hline $\mathrm{O}$ & 49 & 1 & Chuyskoye & Muscovite pegmatite & Muscovite & Mamsko-Chuiskiy \\
\hline $\mathrm{O}$ & 49 & 2 & Vitimskoye & Muscovite pegmatite & Muscovite & Mamsko-Chuiskiy \\
\hline $\mathrm{O}$ & 49 & 3 & Bolshoye Severnoye & Muscovite pegmatite & Muscovite & Mamsko-Chuiskiy \\
\hline $\mathrm{O}$ & 49 & 4 & Lugovka & Muscovite pegmatite & Muscovite & Mamsko-Chuiskiy \\
\hline $\mathrm{O}$ & 49 & 5 & Kolotovskoye & Muscovite pegmatite & Muscovite & Mamsko-Chuiskiy \\
\hline $\mathrm{O}$ & 49 & 6 & $\begin{array}{l}\text { Komsomolsko- } \\
\text { Molodezhnoye }\end{array}$ & Muscovite pegmatite & Muscovite & Mamsko-Chuiskiy \\
\hline $\mathrm{O}$ & 49 & 7 & Synnyrskoye & Magmatic and metasomatic apatite & Apatite & Synnyrskiy \\
\hline $\mathrm{O}$ & 49 & 8 & Kholodninskoye & $\begin{array}{l}\text { Volcanogenic-hydrothermal-sedimentary massive sulfide } \\
\quad \mathrm{Pb}-\mathrm{Zn}( \pm \mathrm{Cu})\end{array}$ & $\mathrm{Pb}, \mathrm{Zn}$ & Olokitskiy \\
\hline $\mathrm{O}$ & 49 & 9 & Sogdiondonskoye & Muscovite pegmatite & Muscovite & Mamsko-Chuiskiy \\
\hline $\mathrm{O}$ & 50 & 1 & Murunskoe & Charoite metasomatite & Charoite & Charskiy \\
\hline $\mathrm{O}$ & 50 & 10 & Sakinskoye & Sediment-hosted $\mathrm{Cu}$ & $\mathrm{Cu}$ & Uguy-Udokanskiy \\
\hline
\end{tabular}




\begin{tabular}{|c|c|c|c|c|c|c|}
\hline Row & Col. & No. & Deposit Name & Mineral Deposit Type & Major Metals & Metallogenic Belt \\
\hline $\mathrm{O}$ & 50 & 11 & Udokanskoye & Sediment-hosted $\mathrm{Cu}$ & $\mathrm{Cu}$ & Uguy-Udokanskiy \\
\hline $\mathrm{O}$ & 50 & 12 & Chineyskoye & Zoned mafic-ultramafic Cr-PGE & $\mathrm{Fe}, \mathrm{Ti}$ & Uguy-Udokanskiy \\
\hline $\mathrm{O}$ & 50 & 13 & Ledyanoe & $\mathrm{Au}$ in shear zone and quartz vein & $\mathrm{Au}$ & Kalar-Stanovoy \\
\hline $\mathrm{O}$ & 50 & 14 & Chineiskoye & Mafic-ultramafic related Ti-Fe (V) & $\mathrm{Ti}, \mathrm{Fe}$ & Uguy-Udokanskiy \\
\hline $\mathrm{O}$ & 50 & 15 & Sulbanskoye & Sediment-hosted $\mathrm{Cu}$ & $\mathrm{Cu}$ & Uguy-Udokanskiy \\
\hline $\mathrm{O}$ & 50 & 16 & Pravo-Ingamakitskoye & Sediment-hosted $\mathrm{Cu}$ & $\mathrm{Cu}$ & Uguy-Udokanskiy \\
\hline $\mathrm{O}$ & 50 & 17 & Burpalinskoye & Sediment-hosted $\mathrm{Cu}$ & $\mathrm{Cu}$ & Uguy-Udokanskiy \\
\hline $\mathrm{O}$ & 50 & 18 & Verkhne-Sakukanskoye & $\mathrm{Au}$ in shear zone and quartz vein & $\mathrm{Au}$ & Muiskiy \\
\hline $\mathrm{O}$ & 50 & 19 & Molodezhnoye & Serpentinite-hosted asbestos & $\begin{array}{l}\text { Chrysotile- } \\
\text { asbestos }\end{array}$ & Baikalo-Muiskiy \\
\hline $\mathrm{O}$ & 50 & 2 & Chertovo Koryto & $\mathrm{Au}$ in black shale & $\mathrm{Au}$ & Tonodskiy \\
\hline $\mathrm{O}$ & 50 & 20 & Kelyanskoye & Carbonate-hosted $\mathrm{Hg}$-Sb & $\mathrm{Hg}$ & Muiskiy \\
\hline $\mathrm{O}$ & 50 & 21 & Katuginskoye & Ta-Nb-REE alkaline metasomatite & $\mathrm{Ta}, \mathrm{Nb}, \mathrm{REE}$ & Uguy-Udokanskiy \\
\hline $\mathrm{O}$ & 50 & 22 & Unkurskoye & Sediment-hosted $\mathrm{Cu}$ & $\mathrm{Cu}$ & Uguy-Udokanskiy \\
\hline $\mathrm{O}$ & 50 & 3 & Vysochaishiy & $\mathrm{Au}$ in black shale & $\mathrm{Au}$ & Bodaibinskiy \\
\hline $\mathrm{O}$ & 50 & 4 & Sukhoy Log & $\mathrm{Au}$ in black shale & $\mathrm{Au}$ & Bodaibinskiy \\
\hline $\mathrm{O}$ & 50 & 5 & Olondo & $\mathrm{Au}$ in shear zone and quartz vein & $\mathrm{Au}$ & West Aldan \\
\hline $\mathrm{O}$ & 50 & 6 & Charskoye & Banded iron formation (BIF, Superior Fe) & $\mathrm{Fe}$ & Uguy-Udokanskiy \\
\hline $\mathrm{O}$ & 50 & 7 & Krasnoye & Sediment-hosted $\mathrm{Cu}$ & $\mathrm{Cu}$ & Uguy-Udokanskiy \\
\hline $\mathrm{O}$ & 50 & 8 & Dogaldynskoe & Au in black shale & $\mathrm{Au}$ & Bodaibinskiy \\
\hline $\mathrm{O}$ & 50 & 9 & Tarynnakh & Banded iron formation (BIF, Superior Fe) & $\mathrm{Fe}, \mathrm{Au}$ & West Aldan \\
\hline $\mathrm{O}$ & 51 & 1 & Seligdar & Apatite carbonatite & $\mathrm{P}$ & Nimnyr \\
\hline $\mathrm{O}$ & 51 & 10 & Usuu & Sediment-hosted $\mathrm{Cu}$ & $\mathrm{Cu}$ & Uguy-Udokan \\
\hline $\mathrm{O}$ & 51 & 11 & Dagda & Banded iron formation (BIF, Superior Fe) & $\mathrm{Fe}, \mathrm{Al}$ & West Aldan \\
\hline $\mathrm{O}$ & 51 & 12 & Pravokabaktanskoe & Au in shear zone and quartz vein & $\mathrm{Au}$ & Kalar-Stanovoy \\
\hline $\mathrm{O}$ & 51 & 2 & Perekatnoe & Piezoquartzite & piezoquartz & Upper Aldan \\
\hline $\mathrm{O}$ & 51 & 3 & Tayozhnoe 2 & Fe skarn & $\mathrm{Fe}$ & Dyos-Leglier \\
\hline $\mathrm{O}$ & 51 & 4 & $\begin{array}{l}\text { Nadyozhnoe, } \\
\text { Fyodorovskoe }\end{array}$ & Phlogopite skarn & $\begin{array}{l}\text { phlogopite, } \\
\text { vermiculite }\end{array}$ & Tympton \\
\hline $\mathrm{O}$ & 51 & 5 & Dyosovskoe & Fe skarn & $\mathrm{Fe}$ & Dyos-Leglier \\
\hline $\mathrm{O}$ & 51 & 6 & Bugarykta & Piezoquartzite & Piezoquartz & Upper Aldan \\
\hline $\mathrm{O}$ & 51 & 7 & Lemochi & $\mathrm{Au}$ in shear zone and quartz vein & $\mathrm{Au}$ & West Aldan \\
\hline $\mathrm{O}$ & 51 & 8 & Nelyuki & Banded iron formation (BIF, Superior Fe) & $\mathrm{Fe}$ & West Aldan \\
\hline $\mathrm{O}$ & 51 & 9 & Kuranakh & Au potassium metasomatite (Kuranakh type) & $\mathrm{Au}$ & Chara-Aldan \\
\hline $\mathrm{O}$ & 52 & 1 & Megyuskan & Phlogopite skarn & $\begin{array}{l}\text { Phlogopite, } \\
\text { vermiculite, } \mathrm{Fe}\end{array}$ & Uchur \\
\hline $\mathrm{O}$ & 52 & 2 & Emeldzhak & Fe skarn & $\mathrm{Fe}$ & Dyos-Leglier \\
\hline $\mathrm{O}$ & 52 & 3 & Atugey & Banded iron formation (BIF, Superior Fe) & $\mathrm{Fe}$ & Davangra-Nalurak \\
\hline $\mathrm{O}$ & 52 & 4 & Olimpiyskoe & Banded iron formation (BIF, Superior Fe) & $\mathrm{Fe}$ & Sutam \\
\hline $\mathrm{O}$ & 53 & 1 & Yur & $\mathrm{Au}$ in shear zone and quartz vein & $\mathrm{Au}$ & Allakh-Yun \\
\hline $\mathrm{O}$ & 53 & 10 & Tas-Yuryakh & Au-Ag epithermal vein & $\mathrm{Au}$ & South Verkhoyansk \\
\hline $\mathrm{O}$ & 53 & 11 & Borong & Sediment-hosted $\mathrm{Cu}$ & $\mathrm{Cu}$ & Sette-Daban \\
\hline $\mathrm{O}$ & 53 & 12 & Malyutka & $\mathrm{Au}$ in shear zone and quartz vein & $\mathrm{Au}$ & Allakh-Yun \\
\hline $\mathrm{O}$ & 53 & 13 & Algaminskoe & Carbonate-hosted Zr (Algoma type) & $\mathrm{Zr}, \mathrm{W}$ & Ingili \\
\hline $\mathrm{O}$ & 53 & 14 & Ingili & $\mathrm{REE}( \pm \mathrm{Ta}, \mathrm{Nb}, \mathrm{Fe})$ carbonatite & REE, $\mathrm{Nb}, \mathrm{Ta}$ & Ingili \\
\hline $\mathrm{O}$ & 53 & 15 & Chad (Mokhovoy) & Mafic-ultramafic related $\mathrm{Cu}-\mathrm{Ni}$-PGE & PGE & Kondyor-Feklistov \\
\hline $\mathrm{O}$ & 53 & 16 & Sigilyakh & Sediment-hosted $\mathrm{Cu}$ & $\mathrm{Cu}$ & Sette-Daban \\
\hline $\mathrm{O}$ & 53 & 17 & Maly Komui & $\mathrm{Cu}( \pm \mathrm{Fe}, \mathrm{Au}, \mathrm{Ag}, \mathrm{Mo})$ skarn & $\mathrm{Cu}$ & Chelasin \\
\hline $\mathrm{O}$ & 53 & 18 & Dzhagdag & Basaltic $\mathrm{Cu}$ (Lake Superior type) & $\mathrm{Cu}$ & Sette-Daban \\
\hline $\mathrm{O}$ & 53 & 19 & Chelasin & Porphyry $\mathrm{Cu}( \pm \mathrm{Au})$ & $\mathrm{Cu}$ & Chelasin \\
\hline $\mathrm{O}$ & 53 & 2 & Duet & $\mathrm{Au}$ in shear zone and quartz vein & $\mathrm{Au}$ & Allakh-Yun \\
\hline
\end{tabular}




\begin{tabular}{|c|c|c|c|c|c|c|}
\hline Row & Col. & No. & Deposit Name & Mineral Deposit Type & Major Metals & Metallogenic Belt \\
\hline $\mathrm{O}$ & 53 & 20 & Kondyor & Zoned mafic-ultramafic Cr-PGE & $\mathrm{Pt}$ & Kondyor-Feklistov \\
\hline $\mathrm{O}$ & 53 & 21 & Avlayakan & Au-Ag epithermal vein & $\mathrm{Au}, \mathrm{Ag}$ & Preddzhugdzhursky \\
\hline $\mathrm{O}$ & 53 & 22 & Klin & Au skarn & $\mathrm{Au}$ & Chara-Aldan \\
\hline $\mathrm{O}$ & 53 & 23 & Krutoy & $\mathrm{Au}$ in shear zone and quartz vein & $\mathrm{Au}$ & Chara-Aldan \\
\hline $\mathrm{O}$ & 53 & 24 & Ulakhan & Au-Ag epithermal vein & $\mathrm{Au}$ & Chara-Aldan \\
\hline $\mathrm{O}$ & 53 & 25 & Ulkanskoe & Ta-Li ongonite & $\mathrm{REE}, \mathrm{Be}, \mathrm{Zr}$ & Verkhneuchur? \\
\hline $\mathrm{O}$ & 53 & 26 & Begundya & Felsic plutonic U-REE & $\mathrm{REE}, \mathrm{Be}, \mathrm{Zr}$ & Verkhneuchur \\
\hline $\mathrm{O}$ & 53 & 27 & Nygvagan-II & REE-Li pegmatite & $\mathrm{Nb}, \mathrm{Ta}$ & Verkhneuchur \\
\hline $\mathrm{O}$ & 53 & 28 & Neozhidannoye & Felsic plutonic U-REE & REE & Verkhneuchur \\
\hline $\mathrm{O}$ & 53 & 29 & Khaykan & Ta-Nb-REE alkaline metasomatite & REE & Verkhneuchur \\
\hline $\mathrm{O}$ & 53 & 3 & Gornoye Ozero & $\mathrm{REE}( \pm \mathrm{Ta}, \mathrm{Nb}, \mathrm{Fe})$ carbonatite & $\mathrm{P}, \mathrm{Nb}, \mathrm{Ta}, \mathrm{REE}$ & Sette-Daban \\
\hline $\mathrm{O}$ & 53 & 30 & Amulican & Ta-Nb-REE alkaline metasomatite & REE & Verkhneuchur \\
\hline $\mathrm{O}$ & 53 & 31 & Algama & Stratiform Zr (Algama type) & $\mathrm{Zr}$ & Ingili \\
\hline $\mathrm{O}$ & 53 & 4 & Urui & Carbonate-hosted Pb-Zn (Mississippi valley type) & $\mathrm{Pb}, \mathrm{Zn}$ & Kyllakh \\
\hline $\mathrm{O}$ & 53 & 5 & Khamna & $\mathrm{REE}( \pm \mathrm{Ta}, \mathrm{Nb}, \mathrm{Fe})$ carbonatite & $\mathrm{REE}, \mathrm{Nb}$ & Sette-Daban \\
\hline $\mathrm{O}$ & 53 & 6 & Yudoma & Polymetallic $(\mathrm{Pb}, \mathrm{Zn}, \mathrm{Ag})$ carbonate-hosted metasomatite & $\mathrm{Zn}$ & Kyllakh \\
\hline $\mathrm{O}$ & 53 & 7 & Pukhanil & Carbonate-hosted $\mathrm{Pb}-\mathrm{Zn}$ (Mississippi valley type) & $\mathrm{Zn}, \mathrm{Pb}$ & Kyllakh \\
\hline $\mathrm{O}$ & 53 & 8 & Lugun & Carbonate-hosted $\mathrm{Pb}-\mathrm{Zn}$ (Mississippi valley type) & $\mathrm{Pb}, \mathrm{Zn}$ & Sette-Daban \\
\hline $\mathrm{O}$ & 53 & 9 & Muromets & $\mathrm{Cu}( \pm \mathrm{Fe}, \mathrm{Au}, \mathrm{Ag}, \mathrm{Mo})$ skarn & $\mathrm{Cu}, \mathrm{Mo}, \mathrm{W}$ & Allakh-Yun \\
\hline $\mathrm{O}$ & 54 & 1 & Larisa & Polymetallic $\mathrm{Pb}-\mathrm{Zn} \pm \mathrm{Cu}( \pm \mathrm{Ag}, \mathrm{Au})$ vein and stockwork & $\mathrm{Zn}$ & Upper Udoma \\
\hline $\mathrm{O}$ & 54 & 2 & Burgali & Porphyry Mo $( \pm \mathrm{W}, \mathrm{Sn}, \mathrm{Bi})$ & Mo, W & Upper Udoma \\
\hline $\mathrm{O}$ & 54 & 3 & $\begin{array}{l}\text { Balaakkalakh, Diring- } \\
\text { Yuryak }\end{array}$ & Sn-W greisen, stockwork, and quartz vein & $\mathrm{Sn}$ & Upper Udoma \\
\hline $\mathrm{O}$ & 54 & 4 & Zhar & $\mathrm{Au}$ in shear zone and quartz vein & $\mathrm{Au}$ & Allakh-Yun \\
\hline $\mathrm{O}$ & 54 & 5 & Dies & $\mathrm{Cu}( \pm \mathrm{Fe}, \mathrm{Au}, \mathrm{Ag}, \mathrm{Mo})$ skarn & $\mathrm{Cu}$ & South Verkhoyansk \\
\hline $\mathrm{O}$ & 54 & 6 & Verkhnenyotskoe & $\mathrm{Au}-\mathrm{Ag}$ epithermal vein & $\mathrm{Au}, \mathrm{Ag}$ & Kukhtuy-Uliya \\
\hline $\mathrm{O}$ & 54 & 7 & Etandzha & Porphyry $\mathrm{Cu}-\mathrm{Mo}( \pm \mathrm{Au}, \mathrm{Ag})$ & $\mathrm{Cu}, \mathrm{Mo}$ & Chelasin \\
\hline $\mathrm{P}$ & 45 & 1 & Eloguiskoye & Volcanogenic-sedimentary $\mathrm{Fe}$ & $\mathrm{Fe}$ & Turukhansk \\
\hline $\mathrm{P}$ & 45 & 2 & Porozhinskoye 1 & Volcanogenic-sedimentary Mn & $\mathrm{Mn}$ & Isakovsk \\
\hline $\mathrm{P}$ & 46 & 1 & Suringdakonskoye & Fe skarn & $\mathrm{Fe}$ & Kyreisko-Tungsk \\
\hline $\mathrm{P}$ & 46 & 10 & Proletarskoye & $\mathrm{Au}$ in shear zone and quartz vein & $\mathrm{Au}$ & Central-Yenisey \\
\hline $\mathrm{P}$ & 46 & 11 & Eldorado & $\mathrm{Au}$ in shear zone and quartz vein & $\mathrm{Au}$ & Central-Yenisey \\
\hline $\mathrm{P}$ & 46 & 12 & Grigorevskoye & Polymetallic $\mathrm{Pb}-\mathrm{Zn} \pm \mathrm{Cu}( \pm \mathrm{Ag}, \mathrm{Au})$ vein and stockwork & $\mathrm{Ag}$ & Central Yenisey \\
\hline $\mathrm{P}$ & 46 & 13 & Enashiminskoye 3 & REE-Li pegmatite & $\mathrm{Sn}, \mathrm{Be}, \mathrm{Li}$ & Tatarsko-Tyradinsk \\
\hline $\mathrm{P}$ & 46 & 14 & Khariuzikhinskoye 2 & REE-Li pegmatite & $\mathrm{Be}$ & Tatarsko-Tyradinsk \\
\hline $\mathrm{P}$ & 46 & 15 & Isakovskoye 2 & Ta-Nb-REE alkaline metasomatite & $\mathrm{Ta}, \mathrm{Nb}, \mathrm{Be}$ & Tatarsko-Tyradinsl \\
\hline $\mathrm{P}$ & 46 & 16 & Khariuzikhinskoye 1 & Volcanogenic $\mathrm{Cu}-\mathrm{Zn}$ massive sulfide (Urals type) & $\mathrm{Cu}, \mathrm{Zn}$ & Isakovsk \\
\hline $\mathrm{P}$ & 46 & 17 & Isakovskoye 1 & Banded iron formation (BIF, Superior Fe) & $\mathrm{Fe}$ & Isakovsk \\
\hline $\mathrm{P}$ & 46 & 18 & Levotyradinskoye & $\mathrm{W} \pm \mathrm{Mo} \pm \mathrm{Be}$ skarn & Be, REE, Sn & Tatarsko-Tyradinsk \\
\hline $\mathrm{P}$ & 46 & 2 & Komdalskoye & Fe skarn & $\mathrm{Fe}$ & Kureisko-Tungsk \\
\hline $\mathrm{P}$ & 46 & 3 & Bakhtinskoye & Fe skarn & $\mathrm{Fe}$ & Kureisko-Tungsk \\
\hline $\mathrm{P}$ & 46 & 4 & Guryevskoye & Sedimentary phosphate & Phosphorite & Unassigned \\
\hline $\mathrm{P}$ & 46 & 5 & Bilchany River & Mafic-ultramafic related $\mathrm{Cu}-\mathrm{Ni}-\mathrm{PGE}$ & $\mathrm{Cu}$ & Kureisko-Tungsk \\
\hline $\mathrm{P}$ & 46 & 6 & Kamyshenskiy Baikitik & Fe skarn & $\mathrm{Fe}$ & Kureisko-Tungsk \\
\hline $\mathrm{P}$ & 46 & 7 & Sukholebyazhinskoye & Sedimentary bauxite & $\mathrm{Al}$ & Unassigned \\
\hline $\mathrm{P}$ & 46 & 8 & Organovskoye & Fe skarn & $\mathrm{Fe}$ & Kureisko-Tungsk \\
\hline $\mathrm{P}$ & 46 & 9 & Sovetskoye & $\mathrm{Au}$ in shear zone and quartz vein & $\mathrm{Au}$ & Central-Yenisey \\
\hline $\mathrm{P}$ & 47 & 1 & Novoye I & Hydrothermal Iceland spar & Island spar & Central Tungussk \\
\hline $\mathrm{P}$ & 47 & 2 & Tychanskoye & Carbonate-hosted $\mathrm{Pb}-\mathrm{Zn}$ (Mississippi valley type) & $\mathrm{Pb}, \mathrm{Zn}$ & Central Tungussk \\
\hline $\mathrm{P}$ & 47 & 3 & Nizhne-Lakur-skoye 1 & Fe skarn & $\mathrm{Fe}$ & Angara-Ilim \\
\hline
\end{tabular}




\begin{tabular}{|c|c|c|c|c|c|c|}
\hline Row & Col. & No. & Deposit Name & Mineral Deposit Type & Major Metals & Metallogenic Belt \\
\hline $\mathrm{P}$ & 48 & 1 & Ilimpeiskoye 1 & Fe skarn & $\mathrm{Fe}$ & Unassigned \\
\hline $\mathrm{P}$ & 48 & 2 & Zeleznaya Gora 1 & Hydrothermal Iceland spar & Island spar & Central Tungussk \\
\hline $\mathrm{P}$ & 48 & 3 & Khrustalnoye & Hydrothermal Iceland spar & Island spar & Central Tungussk \\
\hline $\mathrm{P}$ & 49 & 1 & Mir & Diamond-bearing kimberlite & Diamond & Botuobiya -Markha \\
\hline $\mathrm{P}$ & 49 & 2 & Internatsional'naya & Diamond kimberlite & Diamond & Botuobiya -Markha \\
\hline $\mathrm{P}$ & 53 & 1 & Kurpandzha & Sediment-hosted $\mathrm{Cu}$ & $\mathrm{Cu}$ & Sette-Daban \\
\hline $\mathrm{P}$ & 53 & 10 & Sardana & Carbonate-hosted $\mathrm{Pb}-\mathrm{Zn}$ (Mississippi valley type) & $\mathrm{Pb}, \mathrm{Zn}$ & Kyllakh \\
\hline $\mathrm{P}$ & 53 & 2 & Dzhalkan & Basaltic $\mathrm{Cu}$ (Lake Superior type) & $\mathrm{Cu}$ & Sette-Daban \\
\hline $\mathrm{P}$ & 53 & 3 & Segenyakh & Carbonate-hosted Pb-Zn (Mississippi valley type) & $\mathrm{Pb}, \mathrm{Zn}, \mathrm{CaF} 2$ & Sette-Daban \\
\hline $\mathrm{P}$ & 53 & 4 & Rossomakha & Sediment-hosted $\mathrm{Cu}$ & $\mathrm{Cu}$ & Sette-Daban \\
\hline $\mathrm{P}$ & 53 & 5 & Onello (Lider) & Au in shear zone and quartz vein & $\mathrm{Au}$ & Allakh-Yun \\
\hline $\mathrm{P}$ & 53 & 6 & Svetly & $\mathrm{Au}$ in black shale & $\mathrm{Au}$ & Allakh-Yun (or Khandyga?) \\
\hline $\mathrm{P}$ & 53 & 7 & Bular & Au in shear zone and quartz vein & $\mathrm{Au}$ & Allakh-Yun \\
\hline $\mathrm{P}$ & 53 & 8 & Povorotnoye & REE $( \pm \mathrm{Ta}, \mathrm{Nb}, \mathrm{Fe})$ carbonatite & $\mathrm{Nb}, \mathrm{Ta}$ & Sette-Daban \\
\hline $\mathrm{P}$ & 53 & 9 & Pereval'noe & Carbonate-hosted $\mathrm{Pb}-\mathrm{Zn}$ (Mississippi valley type) & $\mathrm{Pb}, \mathrm{Zn}$ & Kyllakh \\
\hline $\mathrm{P}$ & 54 & 1 & Darpichan & Porphyry Cu-Mo $( \pm \mathrm{Au}, \mathrm{Ag})$ & $\mathrm{Cu}, \mathrm{Mo}$ & Kuydusun \\
\hline $\mathrm{P}$ & 54 & 10 & Tsvetok & $\begin{array}{l}\text { Polymetallic }(\mathrm{Pb}, \mathrm{Zn} \pm \mathrm{Cu}, \mathrm{Ba}, \mathrm{Ag}, \mathrm{Au}) \text { volcanic-hosted } \\
\text { metasomatite }\end{array}$ & $\mathrm{Zn}, \mathrm{Pb}$ & Kukhtuy-Uliya \\
\hline $\mathrm{P}$ & 54 & 11 & Sakyryr & Carbonate-hosted Pb-Zn (Mississippi valley type) & $\mathrm{Zn}, \mathrm{CaF} 2$ & Sette-Daban \\
\hline $\mathrm{P}$ & 54 & 12 & Surkho & Porphyry Sn & $\mathrm{Sn}$ & Kukhtuy-Uliya \\
\hline $\mathrm{P}$ & 54 & 13 & Dochkanakh & Porphyry Mo $( \pm \mathrm{W}, \mathrm{Sn}, \mathrm{Bi})$ & Mo, W & Upper Udoma \\
\hline $\mathrm{P}$ & 54 & 14 & Rozovoye & Porphyry Mo $( \pm \mathrm{W}, \mathrm{Sn}, \mathrm{Bi})$ & Mo & Kukhtuy-Uliya \\
\hline $\mathrm{P}$ & 54 & 15 & Voskhod & Au in shear zone and quartz vein & $\mathrm{Au}$ & South Verkhoyansk \\
\hline $\mathrm{P}$ & 54 & 16 & Novinka & Au in shear zone and quartz vein & $\mathrm{Au}$ & Allakh-Yun \\
\hline $\mathrm{P}$ & 54 & 17 & Zaderzhnoe & Au in shear zone and quartz vein & $\mathrm{Au}$ & Allakh-Yun \\
\hline $\mathrm{P}$ & 54 & 2 & Imtachan & Sn-W greisen, stockwork, and quartz vein & $\mathrm{Pb}, \mathrm{Zn}, \mathrm{Sn}$ & Adycha-Nera \\
\hline $\mathrm{P}$ & 54 & 3 & Senduchen & Clastic-sediment-hosted $\mathrm{Sb}-\mathrm{Au}$ & $\mathrm{As}, \mathrm{Sb}$ & Khandyga \\
\hline $\mathrm{P}$ & 54 & 4 & Upper Menkeche & Polymetallic $\mathrm{Pb}-\mathrm{Zn} \pm \mathrm{Cu}( \pm \mathrm{Ag}, \mathrm{Au})$ vein and stockwork & $\mathrm{Pb}, \mathrm{Zn}, \mathrm{Ag}$ & South Verkhoyansk \\
\hline $\mathrm{P}$ & 54 & 5 & Stibnitovoe & $\mathrm{Ag}$-Sb vein & $\mathrm{Sb}$ & Khandyga \\
\hline $\mathrm{P}$ & 54 & 6 & Levo-Dybin & Granitoid-related $\mathrm{Au}$ vein & $\mathrm{Au}, \mathrm{W}, \mathrm{Bi}$ & South Verkhoyansk \\
\hline $\mathrm{P}$ & 54 & 7 & It-Yuryak & W-Mo-Be greisen, stockwork, and quartz vein & $\mathrm{W}$ & South Verkhoyansk \\
\hline $\mathrm{P}$ & 54 & 8 & Avliya & Sn-W greisen, stockwork, and quartz vein & $\mathrm{Sn}$ & Kuydusun \\
\hline $\mathrm{P}$ & 54 & 9 & Nezhdaninka & Au in shear zone and quartz vein & $\mathrm{Au}, \mathrm{Ag}$ & South Verkhoyansk \\
\hline $\mathrm{Q}$ & 45 & 1 & Nizhny Chopko & Fe skarn & $\mathrm{Fe}$ & Kureisko-Tungsk \\
\hline $\mathrm{Q}$ & 45 & 10 & Turukhanskoye & Volcanogenic-sedimentary Fe & $\mathrm{Fe}$ & Turukhansk \\
\hline $\mathrm{Q}$ & 45 & 11 & Fatyanichinskoye & Metamorphic graphite & Graphite & Kureisko-Tungsk \\
\hline $\mathrm{Q}$ & 45 & 2 & Degen River & Mafic-ultramafic related Cu-Ni-PGE & $\mathrm{Cu}, \mathrm{Co}$ & Kureisko-Tungsk \\
\hline $\mathrm{Q}$ & 45 & 3 & Koly River & Mafic-ultramafic related Cu-Ni-PGE & $\mathrm{Cu}, \mathrm{Co}$ & Kureisko-Tungsk \\
\hline $\mathrm{Q}$ & 45 & 4 & Graviiskoye & Sediment-hosted $\mathrm{Cu}$ & $\mathrm{Cu}$ & Igarsk \\
\hline $\mathrm{Q}$ & 45 & 5 & Severnaya River 2 & Mafic-ultramafic related $\mathrm{Cu}-\mathrm{Ni}$-PGE & $\mathrm{Cu}, \mathrm{Co}$ & Kureisko-Tungsk \\
\hline $\mathrm{Q}$ & 45 & 6 & Kureiskoye 2 & Magmatic graphite & Graphite & Kureisko-Tungsk \\
\hline $\mathrm{Q}$ & 45 & 7 & Sukharikhinskoye & Sediment-hosted $\mathrm{Cu}$ & $\mathrm{Cu}$ & Igarsk \\
\hline Q & 45 & 8 & Kureiskoye 1 & Fe skarn & $\mathrm{Fe}$ & Kureisko-Tungsk \\
\hline $\mathrm{Q}$ & 45 & 9 & Severnaya River 1 & Fe skarn & $\mathrm{Fe}$ & Kureisko-Tungsk \\
\hline Q & 46 & 1 & Ulovny Kamen' & Metamorphic graphite & Graphite & Kureisko-Tungsk \\
\hline $\mathrm{Q}$ & 46 & 2 & Anakitskoye & Fe skarn & $\mathrm{Fe}$ & Kureisko-Tungsk \\
\hline $\mathrm{Q}$ & 46 & 3 & Noginskoye & Metamorphic graphite & Graphite & Kureisko-Tungsk \\
\hline $\mathrm{Q}$ & 47 & 1 & Skala Suslova & Hydrothermal Iceland spar & Island spar & Central Tungussk \\
\hline Q & 47 & 2 & Krutoye (Gonchak) & Hydrothermal Iceland spar & Island spar & Central Tungussk \\
\hline Q & 49 & 1 & Udachnaya & Diamond kimberlite & Diamond & Daldyn-Olenyok \\
\hline
\end{tabular}




\begin{tabular}{|c|c|c|c|c|c|c|}
\hline Row & Col. & No. & Deposit Name & Mineral Deposit Type & Major Metals & Metallogenic Belt \\
\hline $\mathrm{Q}$ & 49 & 2 & Sytykanskaya & Diamond kimberlite & Diamond & Daldyn-Olenyok \\
\hline $\mathrm{Q}$ & 49 & 3 & Yubileinaya & Diamond-bearing kimberlite & Diamond & Daldyn-Olenyok \\
\hline $\mathrm{Q}$ & 49 & 4 & Aikhal & Diamond kimberlite & Diamond & Daldyn-Olenyok \\
\hline $\mathrm{Q}$ & 52 & 1 & Betyugen & Clastic-sediment-hosted Sb-Au & $\mathrm{Sb}$ & Eckyuchu-Billyakh \\
\hline $\mathrm{Q}$ & 52 & 10 & Bochiyskoe & Sn-W greisen, stockwork, and quartz vein & Sn & Verkhoyansk \\
\hline $\mathrm{Q}$ & 52 & 11 & Imtandzha & Sn-W greisen, stockwork, and quartz vein & Sn & West Verkhoyansk \\
\hline $\mathrm{Q}$ & 52 & 12 & Mangazeika 1 & Polymetallic $\mathrm{Pb}-\mathrm{Zn} \pm \mathrm{Cu}( \pm \mathrm{Ag}, \mathrm{Au})$ vein and stockwork & $\mathrm{Pb}, \mathrm{Ag}$ & Vostochno-Verkhoyansk \\
\hline $\mathrm{Q}$ & 52 & 13 & Bezymyannoe & Polymetallic $\mathrm{Pb}-\mathrm{Zn} \pm \mathrm{Cu}( \pm \mathrm{Ag}, \mathrm{Au})$ vein and stockwork & $\mathrm{Ag}, \mathrm{Pb}$ & Eckyuchu-Billyakh \\
\hline $\mathrm{Q}$ & 52 & 14 & Chochimbal & Polymetallic $\mathrm{Pb}-\mathrm{Zn} \pm \mathrm{Cu}( \pm \mathrm{Ag}, \mathrm{Au})$ vein and stockwork & $\mathrm{Au}, \mathrm{Ag}, \mathrm{Pb}$ & West Verkhoyansk \\
\hline $\mathrm{Q}$ & 52 & 15 & Dyabkhanya & Polymetallic $\mathrm{Pb}-\mathrm{Zn} \pm \mathrm{Cu}( \pm \mathrm{Ag}, \mathrm{Au})$ vein and stockwork & $\mathrm{Au}, \mathrm{Ag}$ & Verkhoyansk \\
\hline Q & 52 & 16 & Galochka & $\mathrm{Au}$ in shear zone and quartz vein & $\mathrm{Au}$ & Verkhoyansk \\
\hline $\mathrm{Q}$ & 52 & 17 & Mangazeika 2 & $\mathrm{Au}$ in black shale & $\mathrm{Ag}$ & Verkhoyansk \\
\hline $\mathrm{Q}$ & 52 & 18 & Balbuk & Polymetallic $\mathrm{Pb}-\mathrm{Zn} \pm \mathrm{Cu}( \pm \mathrm{Ag}, \mathrm{Au})$ vein and stockwork & $\mathrm{Pb}$ & Verkhoyansk \\
\hline $\mathrm{Q}$ & 52 & 2 & Iserdek & Clastic sediment-hosted $\mathrm{Hg} \pm \mathrm{Sb}$ & $\mathrm{Hg}$ & Eckyuchu-Billyakh \\
\hline Q & 52 & 3 & Zagadka & Clastic sediment-hosted $\mathrm{Hg} \pm \mathrm{Sb}$ & $\mathrm{Hg}, \mathrm{Sb}$ & Eckyuchu-Billyakh \\
\hline $\mathrm{Q}$ & 52 & 4 & Syncha-I \& II & $\mathrm{Au}$ in shear zone and quartz vein & $\mathrm{Au}$ & Verkhoyansk \\
\hline Q & 52 & 5 & Zvyozdochka & Clastic sediment-hosted $\mathrm{Hg} \pm \mathrm{Sb}$ & $\mathrm{Hg}$ & Eckyuchu-Billyakh \\
\hline Q & 52 & 6 & Kholbolok & Clastic sediment-hosted $\mathrm{Hg} \pm \mathrm{Sb}$ & $\mathrm{Hg}$ & Eckyuchu-Billyakh \\
\hline $\mathrm{Q}$ & 52 & 7 & Kysyltas & Polymetallic $\mathrm{Pb}-\mathrm{Zn} \pm \mathrm{Cu}( \pm \mathrm{Ag}, \mathrm{Au})$ vein and stockwork & $\mathrm{Ag}, \mathrm{Au}, \mathrm{Pb}, \mathrm{Zn}$ & West Verkhoyansk \\
\hline $\mathrm{Q}$ & 52 & 8 & Kuolanda & Polymetallic $\mathrm{Pb}-\mathrm{Zn} \pm \mathrm{Cu}( \pm \mathrm{Ag}, \mathrm{Au})$ vein and stockwork & $\mathrm{Pb}, \mathrm{Zn}, \mathrm{Ag}$ & Verkhoyansk \\
\hline $\mathrm{Q}$ & 52 & 9 & Anomalnoe & Sn-W greisen, stockwork, and quartz vein & $\mathrm{Sn}$ & Yana-Adycha \\
\hline $\mathrm{Q}$ & 53 & 1 & Kysylga & $\mathrm{Ag}-\mathrm{Sb}$ vein & $\mathrm{Au}, \mathrm{Ag}$ & Selennyakh \\
\hline $\mathrm{Q}$ & 53 & 10 & Ulakhan-Egelyakh & Cassiterite-sulfide-silicate vein and stockwork & Sn & Yana-Adycha \\
\hline $\mathrm{Q}$ & 53 & 11 & Delyuvialnoe & Granitoid-related $\mathrm{Au}$ vein & $\mathrm{Au}$ & Adycha-Nera \\
\hline $\mathrm{Q}$ & 53 & 12 & Khoton-Khaya & Cassiterite-sulfide-silicate vein and stockwork & $\mathrm{Sn}$ & Yana-Adycha \\
\hline $\mathrm{Q}$ & 53 & 13 & Ak-Altyn & Au-Ag epithermal vein & $\mathrm{Au}$ & Eckyuchu-Billyakh \\
\hline Q & 53 & 14 & Ilin-Tas & Sn-W greisen, stockwork, and quartz vein & $\mathrm{Sn}$ & Yana-Adycha \\
\hline $\mathrm{Q}$ & 53 & 15 & Alys-Khaya & Sn-W greisen, stockwork, and quartz vein & Sn & Yana-Adycha \\
\hline $\mathrm{Q}$ & 53 & 16 & Burgochan & Sn-W greisen, stockwork, and quartz vein & $\mathrm{Sn}$ & Yana-Adycha \\
\hline $\mathrm{Q}$ & 53 & 17 & Singyami & Clastic sediment-hosted $\mathrm{Hg} \pm \mathrm{Sb}$ & $\mathrm{Hg}$ & Khandyga \\
\hline Q & 53 & 18 & Erikag & Sn-W greisen, stockwork, and quartz vein & Sn & Tompo \\
\hline $\mathrm{Q}$ & 53 & 19 & Prognoz & Polymetallic $\mathrm{Pb}-\mathrm{Zn} \pm \mathrm{Cu}( \pm \mathrm{Ag}, \mathrm{Au})$ vein and stockwork & $\mathrm{Ag}, \mathrm{Pb}$ & Eckyuchu-Billyakh \\
\hline $\mathrm{Q}$ & 53 & 2 & Ege-Khaya & Sn-W greisen, stockwork, and quartz vein & $\mathrm{Sn}, \mathrm{Zn}$ & Yana-Adycha \\
\hline $\mathrm{Q}$ & 53 & 20 & Agylky & $\mathrm{W} \pm \mathrm{Mo} \pm \mathrm{Be}$ skarn & $\mathrm{W}, \mathrm{Cu}$ & Tompo \\
\hline $\mathrm{Q}$ & 53 & 21 & Imnekan & Clastic-sediment-hosted Sb-Au & $\mathrm{Sb}$ & Khandyga \\
\hline $\mathrm{Q}$ & 53 & 22 & Bugdogar & Sn-W greisen, stockwork, and quartz vein & Sn & Yana-Adycha \\
\hline $\mathrm{Q}$ & 53 & 23 & Khunkhada & $\mathrm{W} \pm \mathrm{Mo} \pm \mathrm{Be}$ skarn & W, Sn & Tompo \\
\hline $\mathrm{Q}$ & 53 & 3 & Burgavli & Sn-W greisen, stockwork, and quartz vein & $\mathrm{Sn}$ & Chybagalakh \\
\hline $\mathrm{Q}$ & 53 & 4 & Billyakh & $\mathrm{Ag}$-Sb vein & $\mathrm{Sb}, \mathrm{Au}$ & Eckyuchu-Billyakh \\
\hline $\mathrm{Q}$ & 53 & 5 & Kere-Yuryakh & Sn-W greisen, stockwork, and quartz vein & Sn, W & Chybagalakh \\
\hline $\mathrm{Q}$ & 53 & 6 & Lazo & $\mathrm{Au}$ in shear zone and quartz vein & $\mathrm{Au}$ & Adycha-Nera \\
\hline $\mathrm{Q}$ & 53 & 7 & Sentachan & Clastic-sediment-hosted Sb-Au & $\mathrm{Sb}$ & Taryn \\
\hline Q & 53 & 8 & Kester & Sn-W greisen, stockwork, and quartz vein & $\mathrm{Sn}, \mathrm{Ta}, \mathrm{Nb}, \mathrm{Li}$ & Yana-Adycha \\
\hline $\mathrm{Q}$ & 53 & 9 & Uzlovoe & Clastic-sediment-hosted Sb-Au & $\mathrm{Au}, \mathrm{Sb}$ & Taryn \\
\hline Q & 54 & 1 & Khotoidokh & $\begin{array}{l}\text { Volcanogenic } \mathrm{Zn}-\mathrm{Pb}-\mathrm{Cu} \text { massive sulfide (Kuroko, Altai } \\
\text { types) }\end{array}$ & $\mathrm{Pb}, \mathrm{Zn}, \mathrm{Ag}$ & Erikit \\
\hline $\mathrm{Q}$ & 54 & 2 & Titovskoe & Sn skarn & $\mathrm{B}$ & Chybagalakh \\
\hline $\mathrm{Q}$ & 54 & 3 & Dogdo & Volcanic-hosted Hg & $\mathrm{Hg}$ & Selennyakh \\
\hline $\mathrm{Q}$ & 54 & 4 & Aleshkino & Au in shear zone and quartz vein & $\mathrm{Au}$ & Chybagalakh \\
\hline Q & 54 & 5 & Uchui & Au in shear zone and quartz vein & $\mathrm{Au}$ & Adycha-Nera \\
\hline
\end{tabular}




\begin{tabular}{|c|c|c|c|c|c|c|}
\hline Row & Col. & No. & Deposit Name & Mineral Deposit Type & Major Metals & Metallogenic Belt \\
\hline $\mathrm{Q}$ & 54 & 6 & Darpir & $\mathrm{Au}$ in shear zone and quartz vein & $\mathrm{Au}$ & Adycha-Nera \\
\hline $\mathrm{Q}$ & 54 & 7 & Tumannoe & $\mathrm{Au}$ in shear zone and quartz vein & $\mathrm{Au}$ & Adycha-Nera \\
\hline $\mathrm{Q}$ & 54 & 8 & Seikimyan & Clastic sediment-hosted $\mathrm{Hg} \pm \mathrm{Sb}$ & $\mathrm{Hg}$ & Khandyga \\
\hline $\mathrm{Q}$ & 54 & 9 & Erel & Clastic sediment-hosted $\mathrm{Hg} \pm \mathrm{Sb}$ & $\mathrm{Hg}$ & Khandyga \\
\hline $\mathrm{R}$ & 45 & 1 & Telmi River & Mafic-ultramafic related Cu-Ni-PGE & $\mathrm{Cu}$ & Norilsk \\
\hline $\mathrm{R}$ & 45 & 10 & Chernaya Gora & Mafic-ultramafic related Cu-Ni-PGE & $\begin{array}{l}\mathrm{Cu}, \mathrm{Ni}, \mathrm{Co}, \\
\text { PGE }\end{array}$ & Norilsk \\
\hline $\mathrm{R}$ & 45 & 11 & Ergalakh River & Mafic-ultramafic related $\mathrm{Cu}$-Ni-PGE & $\mathrm{Cu}, \mathrm{Co}$ & Norilsk \\
\hline $\mathrm{R}$ & 45 & 12 & Chabechete Lake & Mafic-ultramafic related Cu-Ni-PGE & $\mathrm{Cu}, \mathrm{Co}$ & Norilsk \\
\hline $\mathrm{R}$ & 45 & 13 & Serebryany Brook & Mafic-ultramafic related Cu-Ni-PGE & $\mathrm{Cu}, \mathrm{Co}$ & Norilsk \\
\hline $\mathrm{R}$ & 45 & 14 & Bolgokhtonskoye & Porphyry Cu-Mo $( \pm \mathrm{Au}, \mathrm{Ag})$ & $\mathrm{Cu}, \mathrm{Mo}$ & Norilsk \\
\hline $\mathrm{R}$ & 45 & 2 & Oktyabrskoye 3 & Mafic-ultramafic related Cu-Ni-PGE & $\begin{array}{l}\mathrm{Cu}, \mathrm{Ni}, \mathrm{Co}, \\
\text { PGE }\end{array}$ & Norilsk \\
\hline $\mathrm{R}$ & 45 & 3 & Imangdinskoye & Mafic-ultramafic related Cu-Ni-PGE & $\begin{array}{l}\mathrm{Cu}, \mathrm{Ni}, \mathrm{Co}, \\
\text { PGE }\end{array}$ & Norilsk \\
\hline $\mathrm{R}$ & 45 & 4 & Talnakh & Mafic-ultramafic related Cu-Ni-PGE & $\mathrm{Cu}, \mathrm{Ni}, \mathrm{Co}, \mathrm{PGE}$ & Norilsk \\
\hline $\mathrm{R}$ & 45 & 5 & Makus & Fe skarn & $\mathrm{Fe}$ & Kureisko-Tungsk \\
\hline $\mathrm{R}$ & 45 & 6 & Norilsk II & Mafic-ultramafic related Cu-Ni-PGE & $\begin{array}{l}\mathrm{Cu}, \mathrm{Ni}, \mathrm{Co}, \\
\text { PGE }\end{array}$ & Norilsk \\
\hline $\mathrm{R}$ & 45 & 7 & Norilsk I & Mafic-ultramafic related Cu-Ni-PGE & $\begin{array}{l}\mathrm{Cu}, \mathrm{Ni}, \mathrm{Co}, \\
\text { PGE }\end{array}$ & Norilsk \\
\hline $\mathrm{R}$ & 45 & 8 & Vologochan River & Mafic-ultramafic related Cu-Ni-PGE & $\mathrm{Cu}, \mathrm{Ni}$ & Norilsk \\
\hline $\mathrm{R}$ & 45 & 9 & Zub-Marksheiderskoye & Mafic-ultramafic related $\mathrm{Cu}-\mathrm{Ni}$-PGE & $\mathrm{Cu}$ & Norilsk \\
\hline $\mathrm{R}$ & 46 & 1 & Namakan River 1 & Mafic-ultramafic related $\mathrm{Cu}$-Ni-PGE & $\mathrm{Ni}$ & Norilsk \\
\hline $\mathrm{R}$ & 46 & 10 & Lama Lake & Mafic-ultramafic related $\mathrm{Cu}$-Ni-PGE & $\mathrm{Cu}$ & Norilsk \\
\hline $\mathrm{R}$ & 46 & 2 & Namakan River 3 & Mafic-ultramafic related Cu-Ni-PGE & $\mathrm{Ni}, \mathrm{Co}$ & Norilsk \\
\hline $\mathrm{R}$ & 46 & 3 & Samasik River & Basaltic Cu (Lake Superior type) & $\mathrm{Cu}$ & Norilsk \\
\hline $\mathrm{R}$ & 46 & 4 & Namakan River 2 & Mafic-ultramafic related $\mathrm{Cu}$-Ni-PGE & $\mathrm{Ni}, \mathrm{Cu}, \mathrm{Co}$ & Norilsk \\
\hline $\mathrm{R}$ & 46 & 5 & Neizvestnoye & Mafic-ultramafic related $\mathrm{Cu}-\mathrm{Ni}-\mathrm{PGE}$ & $\mathrm{Cu}, \mathrm{Ni}, \mathrm{Co}$ & Norilsk \\
\hline $\mathrm{R}$ & 46 & 6 & Chopko River & Mafic-ultramafic related $\mathrm{Cu}$-Ni-PGE & $\mathrm{Cu}$ & Norilsk \\
\hline $\mathrm{R}$ & 46 & 7 & Chapomi River & Mafic-ultramafic related $\mathrm{Cu}-\mathrm{Ni}$-PGE & $\mathrm{Cu}$ & Norilsk \\
\hline $\mathrm{R}$ & 46 & 8 & Arylakh River & Mafic-ultramafic related $\mathrm{Cu}$-Ni-PGE & $\mathrm{Cu}$ & Norilsk \\
\hline $\mathrm{R}$ & 46 & 9 & Arylakhskoye & Basaltic $\mathrm{Cu}$ (Lake Superior type) & $\mathrm{Cu}$ & Norilsk \\
\hline $\mathrm{R}$ & 47 & 1 & Gulinskoye 1 & $\mathrm{Fe}-\mathrm{Ti}( \pm \mathrm{Ta}, \mathrm{Nb}, \mathrm{Fe}, \mathrm{Cu}$, apatite) carbonatite & $\mathrm{Fe}, \mathrm{Ti}$ & Maimecha-Kotuisk \\
\hline $\mathrm{R}$ & 47 & 2 & Gulinskoye 3 & Phlogopite carbonatite & Phlogopite & Maimecha-Kotuisk \\
\hline $\mathrm{R}$ & 47 & 3 & Gulinskoye 2 & $\mathrm{REE}( \pm \mathrm{Ta}, \mathrm{Nb}, \mathrm{Fe})$ carbonatite & $\mathrm{Ta}, \mathrm{Nb}, \mathrm{REE}$ & Maimecha-Kotuisk \\
\hline $\mathrm{R}$ & 48 & 1 & Iriaas 1 & $\mathrm{Fe}-\mathrm{Ti}( \pm \mathrm{Ta}, \mathrm{Nb}, \mathrm{Fe}, \mathrm{Cu}$, apatite) carbonatite & $\mathrm{Fe}, \mathrm{Ti}$ & Maimecha-Kotuisk \\
\hline $\mathrm{R}$ & 48 & 2 & Odikhincha 1 & Phlogopite carbonatite & Phlogopite & Maimecha-Kotuisk \\
\hline $\mathrm{R}$ & 48 & 3 & Kugda 1 & $\mathrm{Fe}-\mathrm{Ti}( \pm \mathrm{Ta}, \mathrm{Nb}, \mathrm{Fe}, \mathrm{Cu}$, apatite) carbonatite & $\mathrm{Fe}, \mathrm{Ti}$ & Maimecha-Kotuisk \\
\hline $\mathrm{R}$ & 48 & 4 & Magan 1 & $\mathrm{Fe}-\mathrm{Ti}( \pm \mathrm{Ta}, \mathrm{Nb}, \mathrm{Fe}, \mathrm{Cu}$, apatite) carbonatite & $\mathrm{Fe}, \mathrm{Ti}$ & Maimecha-Kotuisk \\
\hline $\mathrm{R}$ & 48 & 5 & Bor-Uryakh 1 & $\mathrm{Fe}-\mathrm{Ti}( \pm \mathrm{Ta}, \mathrm{Nb}, \mathrm{Fe}, \mathrm{Cu}$, apatite) carbonatite & $\mathrm{Fe}, \mathrm{Ti}$ & Maimecha-Kotuisk \\
\hline $\mathrm{R}$ & 48 & 6 & Esseiy 1 & $\mathrm{Fe}-\mathrm{Ti}( \pm \mathrm{Ta}, \mathrm{Nb}, \mathrm{Fe}, \mathrm{Cu}$, apatite) carbonatite & $\mathrm{Fe}, \mathrm{Ti}$ & Maimecha-Kotuisk \\
\hline $\mathrm{R}$ & 49 & 1 & Popigay & Impact diamond & Diamond & Popigay \\
\hline $\mathrm{R}$ & 50 & 1 & Tomtor & Weathering crust carbonatite REE-Zr-Nb-Li & $\mathrm{Nb}, \mathrm{REE}, \mathrm{P}$ & Udzha \\
\hline $\mathrm{R}$ & 52 & 1 & Mengeniler & Carbonate-hosted $\mathrm{Pb}-\mathrm{Zn}$ (Mississippi valley type) & $\mathrm{Pb}, \mathrm{Zn}$ & Tuora-Sis \\
\hline $\mathrm{R}$ & 52 & 2 & Kyongdei & Sediment-hosted U & $\mathrm{U}$ & Tuora-Sis \\
\hline $\mathrm{R}$ & 52 & 3 & Nikolaevskoe, Otkrytoe & Au in shear zone and quartz vein & $\mathrm{Au}$ & Verkhoyansk \\
\hline $\mathrm{R}$ & 52 & 4 & Anna-Emeskhin & $\mathrm{Au}$ in shear zone and quartz vein & $\mathrm{Au}$ & Verkhoyansk \\
\hline $\mathrm{R}$ & 52 & 5 & Aga-Kukan & Carbonate-hosted $\mathrm{Pb}-\mathrm{Zn}$ (Mississippi valley type) & $\mathrm{Pb}, \mathrm{Zn}, \mathrm{Cu}$ & Orulgan \\
\hline $\mathrm{R}$ & 52 & 6 & Syugyunyakh-Kende & $\mathrm{Au}$ in shear zone and quartz vein & $\mathrm{Au}$ & Verkhoyansk \\
\hline $\mathrm{R}$ & 52 & 7 & Enichan-Tolono & $\mathrm{Au}$ in shear zone and quartz vein & $\mathrm{Au}$ & Verkhoyansk \\
\hline
\end{tabular}




\begin{tabular}{|c|c|c|c|c|c|c|}
\hline Row & Col. & No. & Deposit Name & Mineral Deposit Type & Major Metals & Metallogenic Belt \\
\hline $\mathrm{R}$ & 53 & 1 & Burguat & $\mathrm{Au}$ in shear zone and quartz vein & $\mathrm{Au}$ & Kular \\
\hline $\mathrm{R}$ & 53 & 10 & Novoe & Granitoid-related Au vein & $\mathrm{Au}$ & Kular \\
\hline $\mathrm{R}$ & 53 & 11 & Argin & Sn-W greisen, stockwork, and quartz vein & $\mathrm{Sn}$ & Chybagalakh \\
\hline $\mathrm{R}$ & 53 & 2 & Sigilyakh & Cassiterite-sulfide-silicate vein and stockwork & Sn & Chokurdak \\
\hline $\mathrm{R}$ & 53 & 3 & Aragochan & Polymetallic $\mathrm{Pb}-\mathrm{Zn} \pm \mathrm{Cu}( \pm \mathrm{Ag}, \mathrm{Au})$ vein and stockwork & $\mathrm{Pb}, \mathrm{Zn}$ & Polousny \\
\hline $\mathrm{R}$ & 53 & 4 & Ulakhan-Sala & Cassiterite-sulfide-silicate vein and stockwork & Sn & Polousny \\
\hline $\mathrm{R}$ & 53 & 5 & Baidakh & Clastic-sediment-hosted Sb-Au & $\mathrm{Sb}$ & Lower Yana \\
\hline $\mathrm{R}$ & 53 & 6 & Dzhuotuk & $\mathrm{Au}$ in shear zone and quartz vein & $\mathrm{Au}$ & Kular \\
\hline $\mathrm{R}$ & 53 & 7 & $\begin{array}{l}\text { Tirekhtyak district } \\
\text { (Nagornoe, Podgornoe, } \\
\text { Poputnoe) }\end{array}$ & Sn-W greisen, stockwork, and quartz vein & Sn, W & Kular \\
\hline $\mathrm{R}$ & 53 & 8 & Kyuchyus & $\mathrm{Ag}$-Sb vein & $\mathrm{Au}, \mathrm{Hg}, \mathrm{Sb}$ & Lower Yana \\
\hline $\mathrm{R}$ & 53 & 9 & Solur & Granitoid-related Au vein & $\mathrm{Au}$ & Kular \\
\hline $\mathrm{R}$ & 54 & 1 & Ukachilkan & Cassiterite-sulfide-silicate vein and stockwork & $\mathrm{Sn}$ & Central Polousny \\
\hline $\mathrm{R}$ & 54 & 2 & Dalnee & Polymetallic $\mathrm{Pb}-\mathrm{Zn} \pm \mathrm{Cu}( \pm \mathrm{Ag}, \mathrm{Au})$ vein and stockwork & $\mathrm{Pb}, \mathrm{Zn}$ & Polousny \\
\hline $\mathrm{R}$ & 54 & 3 & Deputatskoye & Sn-W greisen, stockwork, and quartz vein & $\mathrm{Sn}$ & Central Polousny \\
\hline $\mathrm{R}$ & 54 & 4 & Takalkan & Sn-W greisen, stockwork, and quartz vein & Sn & Central Polousny \\
\hline $\mathrm{R}$ & 54 & 5 & Chibagalakh & Sn skarn & $\mathrm{B}, \mathrm{Sn}$ & Chybagalakh \\
\hline $\mathrm{S}$ & 44 & 1 & Uboininskoye & Carbonate-hosted Hg-Sb & $\mathrm{Hg}$ & Tari-Bigaiy \\
\hline $\mathrm{S}$ & 45 & 1 & Lenivaya River & W-Mo-Be greisen, stockwork, and quartz vein & Mo & North Taimyr \\
\hline $\mathrm{S}$ & 45 & 2 & Rostorguev Island & W-Mo-Be greisen, stockwork, and quartz vein & Mo & North Taimyr \\
\hline $\mathrm{S}$ & 46 & 1 & Kolomeitseva River & W-Mo-Be greisen, stockwork, and quartz vein & Mo & North Taimyr \\
\hline $\mathrm{S}$ & 46 & 2 & Mamont River 2 & Porphyry $\mathrm{Cu}-\mathrm{Mo}( \pm \mathrm{Au}, \mathrm{Ag})$ & Mo & North Taimyr \\
\hline $\mathrm{S}$ & 46 & 3 & Mamont River 1 & W-Mo-Be greisen, stockwork, and quartz vein & Mo & North Taimyr \\
\hline $\mathrm{S}$ & 46 & 4 & Morzhovoye & W-Mo-Be greisen, stockwork, and quartz vein & Mo & North Taimyr \\
\hline $\mathrm{S}$ & 46 & 5 & Ilistaya River & Porphyry $\mathrm{Cu}-\mathrm{Mo}( \pm \mathrm{Au}, \mathrm{Ag})$ & $\mathrm{Cu}$ & North Taimyr \\
\hline $\mathrm{S}$ & 46 & 6 & Shtellinga Cape & REE-Li pegmatite & $\mathrm{Be}$ & Birulinsk \\
\hline $\mathrm{S}$ & 46 & 7 & Geologicheskaya Gryada & Podiform chromite & $\mathrm{Cr}$ & Unassigned \\
\hline $\mathrm{S}$ & 46 & 8 & Izvilistaya River & Carbonate-hosted Hg-Sb & $\mathrm{Hg}$ & Tari-Bigaiy \\
\hline $\mathrm{S}$ & 47 & 1 & Surovoye Lake 1 & Polymetallic $\mathrm{Pb}-\mathrm{Zn} \pm \mathrm{Cu}( \pm \mathrm{Ag}, \mathrm{Au})$ vein and stockwork & $\mathrm{Pb}, \mathrm{Zn}$ & Byrranga \\
\hline $\mathrm{S}$ & 47 & 2 & Oranzhevaya River 1 & $\begin{array}{l}\text { Polymetallic }(\mathrm{Pb}, \mathrm{Zn} \pm \mathrm{Cu}, \mathrm{Ba}, \mathrm{Ag}, \mathrm{Au}) \text { volcanic-hosted } \\
\text { metasomatite }\end{array}$ & $\mathrm{Pb}, \mathrm{Ag}$ & Byrranda \\
\hline $\mathrm{S}$ & 47 & 3 & Partizanskoye & Polymetallic $\mathrm{Pb}-\mathrm{Zn} \pm \mathrm{Cu}( \pm \mathrm{Ag}, \mathrm{Au})$ vein and stockwork & $\mathrm{Pb}, \mathrm{Zn}$ & Byrranga \\
\hline $\mathrm{S}$ & 48 & 1 & Nirkaika-Tari 1 & Polymetallic $\mathrm{Pb}-\mathrm{Zn} \pm \mathrm{Cu}( \pm \mathrm{Ag}, \mathrm{Au})$ vein and stockwork & $\mathrm{Pb}$ & Byrranga \\
\hline $\mathrm{S}$ & 48 & 2 & Malachai-Tari 1 & Polymetallic $\mathrm{Pb}-\mathrm{Zn} \pm \mathrm{Cu}( \pm \mathrm{Ag}, \mathrm{Au})$ vein and stockwork & $\mathrm{Cu}$ & Byrranga \\
\hline $\mathrm{S}$ & 48 & 3 & Kungasalakh 1 & Mafic-ultramafic related Cu-Ni-PGE & $\mathrm{Cu}$ & Byrranga \\
\hline $\mathrm{T}$ & 46 & 1 & Birulinskoye & REE-Li pegmatite & $\mathrm{Be}$ & Birulinsk \\
\hline $\mathrm{T}$ & 47 & 1 & Lagerny Cape & Mafic-ultramafic related $\mathrm{Cu}-\mathrm{Ni}$-PGE & $\mathrm{Cu}$ & Severo-Zemelsk \\
\hline $\mathrm{T}$ & 47 & 2 & Studeninskoye & Granitoid-related Au vein & $\mathrm{Au}$ & Unassigned \\
\hline $\mathrm{T}$ & 47 & 3 & Ozernaya River & Mafic-ultramafic related $\mathrm{Cu}-\mathrm{Ni}$-PGE & $\mathrm{Cu}$ & Severo-Zemelsk \\
\hline $\mathrm{T}$ & 47 & 4 & Olovyanny Cape & W-Mo-Be greisen, stockwork, and quartz vein & Sn & North Taimyr \\
\hline $\mathrm{T}$ & 48 & 1 & Kunarskoye 1 & Granitoid-related $\mathrm{Au}$ vein & $\mathrm{Au}$ & Unassigned \\
\hline $\mathrm{T}$ & 48 & 2 & Lodochnikov Plateau 1 & W-Mo-Be greisen, stockwork, and quartz vein & Mo & North Taimyr \\
\hline $\mathrm{U}$ & 47 & 1 & Proliv Krasnoy Armii & Polymetallic $\mathrm{Pb}-\mathrm{Zn} \pm \mathrm{Cu}( \pm \mathrm{Ag}, \mathrm{Au})$ vein and stockwork & $\mathrm{Pb}, \mathrm{Zn}$ & Byrranga \\
\hline
\end{tabular}




\begin{tabular}{|l|l|l|l|l|l|}
\hline Row & Col. & No. & Deposit Name & Deposit Type & Major Metals \\
\hline
\end{tabular}

Table 3. Summary table for selected significant placer districts of Northeast Asia. Districts listed in numerical order by map row and map column for quadrants of latitude and longitude.

\begin{tabular}{|c|c|c|c|c|c|}
\hline $\mathrm{H}$ & 52 & 01 & Ibusuki & Placer Fe & $\mathrm{Fe}$ \\
\hline $\mathrm{I}$ & 52 & 01 & Kunisaki & Placer Fe & $\mathrm{Fe}$ \\
\hline I & 53 & 01 & Yonago & Placer Fe & $\mathrm{Fe}$ \\
\hline I & 53 & 02 & Iioka & Placer Fe & $\mathrm{Fe}$ \\
\hline $\mathrm{J}$ & 51 & 01 & Toudaohe area, Fuxian County & Placer diamond & Diamond \\
\hline $\mathrm{J}$ & 51 & 02 & $\begin{array}{l}\text { Jiaodong(East part of Shandong } \\
\text { Province) }\end{array}$ & Placer $\mathrm{Au}$ & $\mathrm{Au}$ \\
\hline $\mathrm{J}$ & 52 & 01 & Hanjin & Placer Au & $\mathrm{Au}$ \\
\hline $\mathrm{J}$ & 52 & 02 & Sungnam & Placer Au & $\mathrm{Au}$ \\
\hline $\mathrm{J}$ & 52 & 03 & Asan Bay & Placer Au & $\mathrm{Au}$ \\
\hline $\mathrm{J}$ & 52 & 04 & Kumma-chon & Placer $\mathrm{Au}$ & $\mathrm{Au}$ \\
\hline $\mathrm{J}$ & 52 & 05 & Musim-chon & Placer $\mathrm{Au}$ & $\mathrm{Au}$ \\
\hline $\mathrm{J}$ & 52 & 06 & Koseong & Placer Th & $\mathrm{Th}$ \\
\hline $\mathrm{J}$ & 54 & 01 & Kitakami & Placer and paleoplacer $\mathrm{Au}$ & $\mathrm{Au}$ \\
\hline $\mathrm{J}$ & 54 & 02 & Nishimikawa & Placer and paleoplacer $\mathrm{Au}$ & $\mathrm{Au}$ \\
\hline $\mathrm{K}$ & 48 & 01 & Khar Morit & Placer Sn & $\mathrm{SnO} 2$ \\
\hline $\mathrm{K}$ & 49 & 01 & Yinshan, Inner Mongolia & Placer $\mathrm{Au}$ & $\mathrm{Au}$ \\
\hline $\mathrm{K}$ & 49 & 02 & Kuji & Placer Fe & $\mathrm{Fe}$ \\
\hline $\mathrm{K}$ & 50 & 01 & Gaositai & Placer PGE & $\mathrm{Pt}$ \\
\hline $\mathrm{K}$ & 50 & 02 & Yanliao & Placer Au & $\mathrm{Au}$ \\
\hline $\mathrm{K}$ & 51 & 01 & Kamikita & Placer Fe & $\mathrm{Fe}$ \\
\hline $\mathrm{K}$ & 51 & 02 & $\begin{array}{l}\text { Liaodong(Eastern Liaoning } \\
\text { Province) }\end{array}$ & Placer Au & $\mathrm{Au}$ \\
\hline $\mathrm{K}$ & 52 & 01 & $\begin{array}{l}\text { Hunchun-Huadian, Jilin } \\
\text { Province }\end{array}$ & Placer $\mathrm{Au}$ and paleoplacer $\mathrm{Au}$ & $\mathrm{Au}$ \\
\hline $\mathrm{K}$ & 54 & 01 & Horokanai & Placer PGE & $\mathrm{Pt}, \mathrm{Cr}, \mathrm{Au}$ \\
\hline $\mathrm{K}$ & 54 & 02 & Shirokingawa & Placer PGE & $\mathrm{Pt}, \mathrm{Au}$ \\
\hline $\mathrm{K}$ & 54 & 03 & Imagane & Placer and paleoplacer $\mathrm{Au}$ & $\mathrm{Au}$ \\
\hline $\mathrm{K}$ & 54 & 04 & Funkawan & Placer Fe & $\mathrm{Fe}$ \\
\hline $\mathrm{K}$ & 54 & 05 & Matsumae & Placer and paleoplacer $\mathrm{Au}$ & $\mathrm{Au}$ \\
\hline $\mathrm{L}$ & 45 & 01 & Altai, Xinjiang & Placer $\mathrm{Au}$ & $\mathrm{Au}$ \\
\hline $\mathrm{L}$ & 47 & 01 & Dovont & Placer $\mathrm{Au}$ & $\mathrm{Au}$ \\
\hline $\mathrm{L}$ & 48 & 01 & Uyanga-Taragt & Placer $\mathrm{Au}$ & $\mathrm{Au}$ \\
\hline $\mathrm{L}$ & 48 & 02 & Baruun-Urt & Placer Au & $\mathrm{Au}$ \\
\hline $\mathrm{L}$ & 48 & 03 & Janchivlan & Placer Sn & $\mathrm{SnO} 2$ \\
\hline $\mathrm{L}$ & 48 & 04 & Baga Gazar & Placer Sn & $\mathrm{SnO} 2$ \\
\hline $\mathrm{L}$ & 49 & 01 & Modot & Placer Sn & $\mathrm{SnO} 2$ \\
\hline $\mathrm{L}$ & 52 & 01 & Jiayin & Placer $\mathrm{Au}$ & $\mathrm{Au}$ \\
\hline $\mathrm{L}$ & 52 & 02 & Huanan & Placer Au & $\mathrm{Au}$ \\
\hline $\mathrm{L}$ & 52 & 03 & Dongning & Placer $\mathrm{Au}$ & $\mathrm{Au}$ \\
\hline $\mathrm{L}$ & 54 & 01 & Teshio & Placer PGE & PGE, Cr, Au \\
\hline $\mathrm{L}$ & 54 & 02 & Esashi & Placer and paleoplacer $\mathrm{Au}$ & $\mathrm{Au}$ \\
\hline $\mathrm{L}$ & 54 & 03 & Il'inka River & Placer Au & $\mathrm{Au}$ \\
\hline $\mathrm{L}$ & 54 & 04 & Koppi-Nelman & Placer Ti & $\mathrm{Ti}$ \\
\hline $\mathrm{M}$ & 46 & 01 & Burgastain-gol & Placer $\mathrm{Au}$ & $\mathrm{Au}$ \\
\hline $\mathrm{M}$ & 48 & 01 & Yoroo-gol & Placer Au & $\mathrm{Au}$ \\
\hline $\mathrm{M}$ & 48 & 02 & Sharyn-gol-Bukhlei & Placer Au & $\mathrm{Au}$ \\
\hline $\mathrm{M}$ & 48 & 03 & Boroo-Zuunmod & Placer Au & $\mathrm{Au}$ \\
\hline $\mathrm{M}$ & 48 & 04 & Zaamar & Placer Au & $\mathrm{Au}$ \\
\hline $\mathrm{M}$ & 49 & 01 & Baljiin-gol & Placer Au & $\mathrm{Au}$ \\
\hline M & 49 & 02 & Terelj & Placer $\mathrm{Au}$ & $\mathrm{Au}$ \\
\hline $\mathrm{M}$ & 49 & 03 & Turgen & Placer Au & $\mathrm{Au}$ \\
\hline M & 49 & 04 & Narsynkhondlon & Placer $\mathrm{Au}$ & $\mathrm{Au}$ \\
\hline $\mathrm{M}$ & 49 & 05 & Deed Kumiir & Placer Sn & $\mathrm{SnO} 2$ \\
\hline $\mathrm{M}$ & 49 & 06 & Zuuntarts & Placer Sn & $\mathrm{SnO} 2$ \\
\hline M & 52 & 01 & $\begin{array}{l}\text { Malokhingansky (Malokhingan) } \\
\text { district }\end{array}$ & Placer Au & $\mathrm{Au}$ \\
\hline $\mathrm{M}$ & 52 & 02 & $\begin{array}{l}\text { Blagoveshchensk-Svobodnensky } \\
\text { district }\end{array}$ & Placer $\mathrm{Au}$ & $\mathrm{Au}$ \\
\hline $\mathrm{M}$ & 52 & 03 & Turansky district & Placer Au & $\mathrm{Au}$ \\
\hline $\mathrm{M}$ & 52 & 04 & Huma & Placer Au & $\mathrm{Au}$ \\
\hline $\mathrm{M}$ & 54 & 01 & Oemku & Placer Au & $\mathrm{Au}$ \\
\hline $\mathrm{M}$ & 54 & 02 & Langeriiskoe & Placer Au & $\mathrm{Au}$ \\
\hline
\end{tabular}




\begin{tabular}{|l|l|l|l|l|l|}
\hline Row & Col. & No. & Deposit Name & Deposit Type & Major Metals \\
\hline
\end{tabular}

\begin{tabular}{|c|c|c|c|c|c|}
\hline $\mathrm{N}$ & 51 & 01 & Verkhnegilyui & Placer $\mathrm{Au}$ & $\mathrm{Au}$ \\
\hline $\mathrm{N}$ & 51 & 02 & Svrednenyu Kazhinsky & Placer Au & $\mathrm{Au}$ \\
\hline $\mathrm{N}$ & 51 & 03 & Verkhneamursky & Placer Au & $\mathrm{Au}$ \\
\hline $\mathrm{N}$ & 51 & 04 & Gonzhinsky (Gonzha) & Placer $\mathrm{Au}$ & $\mathrm{Au}$ \\
\hline $\mathrm{N}$ & 51 & 05 & Mohe & Placer Au & $\mathrm{Au}$ \\
\hline $\mathrm{N}$ & 52 & 01 & Verkhnezeisky & Placer Au & $\mathrm{Au}$ \\
\hline $\mathrm{N}$ & 52 & $02 \mathrm{a}$ & Dambuki-Part A & Placer $\mathrm{Au}$ & $\mathrm{Au}$ \\
\hline $\mathrm{N}$ & 52 & $02 \mathrm{~b}$ & Dambuki-Part B & Placer $\mathrm{Au}$ & $\mathrm{Au}$ \\
\hline $\mathrm{N}$ & 52 & 03 & Dzhagdy & Placer $\mathrm{Au}$ & $\mathrm{Au}$ \\
\hline $\mathrm{N}$ & 52 & $04 a$ & Zeiya-Selemdzha-Part A & Placer $\mathrm{Au}$ & $\mathrm{Au}$ \\
\hline $\mathrm{N}$ & 52 & $04 \mathrm{~b}$ & Zeiya-Selemdzha-Part B & Placer Au & $\mathrm{Au}$ \\
\hline $\mathrm{N}$ & 52 & $04 \mathrm{c}$ & Zeiya-Selemdzha-Part C & Placer Au & $\mathrm{Au}$ \\
\hline $\mathrm{N}$ & 53 & 01 & Verkhne-Selemdzha & Placer $\mathrm{Au}$ & $\mathrm{Au}$ \\
\hline $\mathrm{N}$ & 53 & 02 & Sofiiskoe & Placer Au & $\mathrm{Au}$ \\
\hline $\mathrm{N}$ & 53 & 03 & Kerbi & Placer $\mathrm{Au}$ & $\mathrm{Au}$ \\
\hline $\mathrm{N}$ & 54 & 01 & Ulskoe & Placer Au & $\mathrm{Au}$ \\
\hline $\mathrm{N}$ & 54 & 02 & Kolchanskoe & Placer $\mathrm{Au}$ & $\mathrm{Au}$ \\
\hline $\mathrm{N}$ & 54 & 03 & Kherpuchinskoe & Placer $\mathrm{Au}$ & $\mathrm{Au}$ \\
\hline $\mathrm{N}$ & 54 & 04 & Oktyabrskoe & Placer $\mathrm{Au}$ & $\mathrm{Au}$ \\
\hline $\mathrm{N}$ & 54 & 05 & Schmidtovskoe & Placer $\mathrm{Au}$ & $\mathrm{Au}$ \\
\hline $\mathrm{O}$ & 51 & 01 & Nizhnenyukzhinsky & Placer $\mathrm{Au}$ & $\mathrm{Au}$ \\
\hline $\mathrm{O}$ & 53 & 01 & Kurun-Uryakh & Placer Au & $\mathrm{Au}$ \\
\hline $\mathrm{O}$ & 53 & 02 & Kondyor & Placer PGE & PGE \\
\hline $\mathrm{O}$ & 53 & 03 & Sash-Yular & Placer Ti & $\mathrm{Ti}$ \\
\hline $\mathrm{O}$ & 54 & 01 & Okhotsk & Placer Au & $\mathrm{Au}$ \\
\hline $\mathrm{P}$ & 54 & 01 & Allakh-Yun & Placer $\mathrm{Au}$ & $\mathrm{Au}$ \\
\hline $\mathrm{Q}$ & 52 & 01 & Verkhoyansk & Placer Au & $\mathrm{Au}$ \\
\hline $\mathrm{Q}$ & 53 & 01 & Verkhne-Yansky & Placer Sn & $\mathrm{Sn}$ \\
\hline $\mathrm{Q}$ & 53 & 02 & Adychan & Placer $\mathrm{Au}$ & $\mathrm{Au}$ \\
\hline Q & 54 & 01 & Verkhne-Indigirsky & Placer $\mathrm{Au}$ & $\mathrm{Au}$ \\
\hline $\mathrm{R}$ & 53 & 01 & Kular & Placer Au & $\mathrm{Au}$ \\
\hline $\mathrm{R}$ & 54 & 01 & Polousnensky & Placer Sn & $\mathrm{Sn}$ \\
\hline $\mathrm{R}$ & 54 & 02 & Khatynnak-Sala & Placer $\mathrm{Au}$ & $\mathrm{Au}$ \\
\hline
\end{tabular}




\begin{tabular}{|c|c|c|c|c|c|}
\hline $\begin{array}{l}\text { Name } \\
\text { (Symbol) }\end{array}$ & $\begin{array}{l}\text { Mineral Deposit Models } \\
\text { (Major Deposits) }\end{array}$ & Country, Region & $\begin{array}{l}\text { Unit or Strucutre } \\
\text { Related to Origin of } \\
\text { Metallogenic Belt }\end{array}$ & Age Range of Metallogenic Belt & $\begin{array}{l}\text { Tectonic Event for Origin of Metallogenic Belt. } \\
\text { Comments }\end{array}$ \\
\hline
\end{tabular}

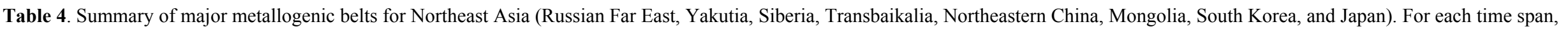
metallogenic belts are listed from west to east, progressing from north to south.

\begin{tabular}{|c|c|c|c|c|c|}
\hline \multicolumn{6}{|c|}{ ARCHEAN (> 2500 Ma) METALLOGENIC BELTS } \\
\hline $\begin{array}{l}\text { West Aldan } \\
\text { (WA) }\end{array}$ & $\begin{array}{l}\text { Banded iron formation (BIF, } \\
\text { Algoma Fe) } \\
\text { (Charskoye, Tarynnakh, } \\
\text { Nelyuki, Dagda, Sulumatskoye, } \\
\text { Severnoye and Yuzhnoye } \\
\text { NizhneSakukan, } \\
\text { Sakukannyrskoye and Oleng- } \\
\text { Turritakhskoye); } \\
\text { Au in shear zone and quartz } \\
\text { vein } \\
\text { (Lemochi, Olondo) }\end{array}$ & $\begin{array}{l}\text { Russia, Southern } \\
\text { Yakutia }\end{array}$ & $\begin{array}{l}\text { West Aldan terrane } \\
\text { (Granite-greenstone). }\end{array}$ & $\begin{array}{l}\text { Archean to Paleoproterozoic. } \\
\text { Metavolcanic and sedimentary } \\
\text { rocks interlayered with BIF have } \\
\text { isotopic ages of } 2.7 \text { to } 3.2 \mathrm{Ga} \text {. } \\
\text { Age of Au occurrences is Late } \\
\text { Archean to Paleoproterozoic. }\end{array}$ & $\begin{array}{l}\text { Belt interpreted as forming in back-arc basin and (or) } \\
\text { island arc. Au occurrences mainly in the shear zones } \\
\text { cutting metabasalt, amphibilite, and ultramafic rock. } \\
\text { Shear zones formed during amalgamation of terranes or } \\
\text { during later tectonic events. BIF (magnetite quartzite) } \\
\text { forms stratiform layers and lenses in metabasalt and } \\
\text { amphibolite, and local siliceous metavolcanic rock and } \\
\text { schist. }\end{array}$ \\
\hline $\begin{array}{l}\text { Sutam } \\
(\mathrm{St})\end{array}$ & $\begin{array}{l}\text { Banded iron formation (BIF, } \\
\text { Algoma Fe) } \\
\text { (Olimpiyskoe) }\end{array}$ & $\begin{array}{l}\text { Russia, Southern } \\
\text { Yakutia }\end{array}$ & $\begin{array}{l}\text { Central Aldan } \\
\text { superterrane (Granulite- } \\
\text { orthogneiss) containing } \\
\text { Sutam terrane with high-T } \\
\text { and high-P granulites. }\end{array}$ & $\begin{array}{l}\text { Archean. Gneiss in Sutam block } \\
\text { has isotopic age of } 2.5 \text { to } 3.0 \mathrm{Ga} \text {. }\end{array}$ & $\begin{array}{l}\text { Two rock groups with BIF occur in belt. (1) Magnetite- } \\
\text { hypersthene and magnetite-pyroxene gneiss interbedded } \\
\text { with amphibole-pyroxene and magnetite-pyroxen- } \\
\text { plagioclase schist. BIF consists of magnetite and } \\
\text { hypersthene-magnetite quartzite occur in outer part of an } \\
\text { antiform. (2) Feldspar quartzite interlayered with garnet- } \\
\text { and sillimanite-bearing schist with diopside calciphyre. } \\
\text { Also occurring are magnetite-hypersthene and garnet- } \\
\text { magnetite hypersthene layers. }\end{array}$ \\
\hline $\begin{array}{l}\text { Sharizhalgai } \\
\text { skiy } \\
(\mathrm{Shz})\end{array}$ & $\begin{array}{l}\text { Banded iron formation (BIF, } \\
\text { Algoma Fe); } \\
\text { Talc (magnesite) replacement } \\
\text { (Sosnovy Baits, Baikalskoye, } \\
\text { Savinskoye) }\end{array}$ & $\begin{array}{l}\text { Russia, Southern- } \\
\text { Eastern Siberia } \\
\text { (East Sayan) }\end{array}$ & $\begin{array}{l}\text { Sharizhalgay terrane } \\
\text { (Tonalite-trondhjemite } \\
\text { gneiss) and Onot terrane } \\
\text { (Granite-greenstone) (too } \\
\text { small to show on } 10 \mathrm{M} \\
\text { scale map) }\end{array}$ & $\begin{array}{l}\text { Archean. Sharyzhalgay series has } \\
\mathrm{U}-\mathrm{Pb}, \mathrm{Rb}-\mathrm{Sr}, \mathrm{Sm}-\mathrm{Nd} \text { isotopic } \\
\text { ages of } 2.42 \text { to } 3.12 \mathrm{Ga} \text {. } \\
\text { Sedimentary rocks in Onot terrane } \\
\text { are Paleoproterozoic. }\end{array}$ & $\begin{array}{l}\text { Some deposits (Kitoy group and Baikalskoye deposit) } \\
\text { are Archean sequences. Others (Onot group - Sosnovy } \\
\text { Baits deposits) are Proterozoic. Layering in ferruginous } \\
\text { quartzite and occurrence in two-pyroxene schists are } \\
\text { interpreted as derived from ferruginous volcanic and } \\
\text { sedimentary rock sequences. }\end{array}$ \\
\hline $\begin{array}{l}\text { Yanbei } \\
(\mathrm{YB})\end{array}$ & $\begin{array}{l}\text { Metamorphic graphite } \\
\text { (Xinghe) }\end{array}$ & Northern China & $\begin{array}{l}\text { Sino-Korean Craton - } \\
\text { Erduosi terrane } \\
\text { (Granulite-paragneiss) }\end{array}$ & Interpreted as Late Archean. & $\begin{array}{l}\text { Deposits hosted in shallow marine clastic and carbonate } \\
\text { sedimentary rocks that formed in a passive Late Archean } \\
\text { continental margin and were metamorphosed to } \\
\text { granulite facies. Host rocks are part of the Late Archean } \\
\text { Upper Jining Group that consists of a khondalite series. }\end{array}$ \\
\hline $\begin{array}{l}\text { Jidong } \\
\text { (JD) }\end{array}$ & $\begin{array}{l}\text { Banded iron formation (BIF, } \\
\text { Algoma Fe) (Shuichang); } \\
\text { Au in shear zone and quartz } \\
\text { vein (Jinchangyu) }\end{array}$ & Northern China & $\begin{array}{l}\text { Sino-Korean Craton - } \\
\text { West Liaoning-Hebei- } \\
\text { Shanxi terrane (Granulite- } \\
\text { orthogneiss) }\end{array}$ & $\begin{array}{l}\text { Archean for BIF deposits that } \\
\text { have } \mathrm{Rb}-\mathrm{Sr} \text { isotopic age greater } \\
\text { than } 3,500 \mathrm{Ma} \text {. Proterozoic or } \\
\text { younger for Au deposits in shear } \\
\text { and retrograde metamorphic } \\
\text { zones with isotopic ages of } 2.5 \text { to } \\
2.6 \mathrm{Ga} ., 1.7 \text { to } 1.8 \mathrm{Ga} \text {., or } \\
\text { younger. }\end{array}$ & $\begin{array}{l}\text { BIF interpreted as forming in volcanic and } \\
\text { sedimentation basin along an unstable proto-continental } \\
\text { margin, or in fragment of Archean craton. Au deposits } \\
\text { interpreted as forming during retrograde metamorphism } \\
\text { to greenschist facies. }\end{array}$ \\
\hline
\end{tabular}




\begin{tabular}{|c|c|c|c|c|c|}
\hline $\begin{array}{l}\text { Name } \\
\text { (Symbol) }\end{array}$ & $\begin{array}{l}\text { Mineral Deposit Models } \\
\text { (Major Deposits) }\end{array}$ & Country, Region & $\begin{array}{l}\text { Unit or Strucutre } \\
\text { Related to Origin of } \\
\text { Metallogenic Belt }\end{array}$ & Age Range of Metallogenic Belt & $\begin{array}{l}\text { Tectonic Event for Origin of Metallogenic Belt. } \\
\text { Comments }\end{array}$ \\
\hline $\begin{array}{l}\text { Liaoji } \\
(\mathrm{LJ})\end{array}$ & $\begin{array}{l}\text { Banded iron formation (BIF, } \\
\text { Algoma Fe) (Gongchangling); } \\
\text { Volcanogenic } \mathrm{Zn}-\mathrm{Pb}-\mathrm{Cu} \\
\text { massive sulfide (Hongtoushan); } \\
\text { Au in shear zone and quartz } \\
\text { vein (Jiapigou) }\end{array}$ & $\begin{array}{l}\text { Northeastern } \\
\text { China }\end{array}$ & $\begin{array}{l}\text { Sino-Korean Craton, Jilin- } \\
\text { Liaoning-East Shandong } \\
\text { terrane }\end{array}$ & $\begin{array}{l}\text { Late Archean. Metamorphic age } \\
\text { of the Anshan Group hosting BIF } \\
\text { is } 2,500 \text { to } 2,650 \text { Ma. Isotopic age } \\
\text { of BIF units probably older than } \\
2,800 \mathrm{Ma} \text {. U-Pb zircon isotopic } \\
\text { age for trondhjemite (mylonite) is } \\
3,804 \mathrm{Ma} \text {. }\end{array}$ & $\begin{array}{l}\text { Host greenstone belt in Northern Liaoning (Hunbei) area } \\
\text { interpreted as forming in an active continental margin } \\
\text { whereas greenstone belts in Anshan-Benxi and Jiapigou } \\
\text { areas interpreted as forming in oceanic rifts along a } \\
\text { continental margin. Au deposits interpreted as forming } \\
\text { during retrograde metamorphism to greenschist facies. }\end{array}$ \\
\hline \multicolumn{6}{|c|}{ PALEOPROTEROZOIC (2500 to 1600 Ma) METALLOGENIC BELTS } \\
\hline $\begin{array}{l}\text { Uguy- } \\
\text { Udokanskiy } \\
\text { (UU) }\end{array}$ & $\begin{array}{l}\text { Zoned mafic-ultramafic Cr- } \\
\text { PGE; } \\
\text { Sediment-hosted Cu; Ta-Nb- } \\
\text { REE alkaline metasomatite; } \\
\text { (Chineyskoye; Udokanskoye, } \\
\text { Pravo-Ingamakit, Sakinskoye, } \\
\text { Sulbanskoye, Katuginskoye) }\end{array}$ & $\begin{array}{l}\text { Russia, Southern } \\
\text { Yakutia }\end{array}$ & $\begin{array}{l}\text { West Aldan terrane } \\
\text { (Granite-greenstone) }\end{array}$ & $\begin{array}{l}\text { Paleoproterozoic. Cu sandstone in } \\
\text { Udokan deposit is } 2.2 \text { to } 1.8 \mathrm{Ga} \text {. } \\
\mathrm{Ta}, \mathrm{Nb}, \mathrm{REE} \text { alkaline } \\
\text { metasomatite deposits age is } 2.0 \\
\text { to } 1.6 \mathrm{Ga}\end{array}$ & $\begin{array}{l}\mathrm{Cr} \text { and PGEdeposits in zoned mafic-ultramafic plutons } \\
\text { and } \mathrm{Cu} \text { in the sedimentary rocks interpreted as forming } \\
\text { along a passive continental-margin rift. Ta-Nb-REE } \\
\text { alkaline metasomatite deposits interpreted as forming } \\
\text { during later collision and formation of anatectic granite. }\end{array}$ \\
\hline $\begin{array}{l}\text { Kalar- } \\
\text { Stanovoy } \\
(\mathrm{KS})\end{array}$ & $\begin{array}{l}\text { Au in shear zone and quartz } \\
\text { vein (Ledyanoe, Namark, } \\
\text { Pravokabaktanskoe) }\end{array}$ & $\begin{array}{l}\text { Russia, Southern } \\
\text { Yakutia }\end{array}$ & $\begin{array}{l}\text { Veins in Kalar tectonic } \\
\text { melange zone }\end{array}$ & $\begin{array}{l}\text { Interpreted as Paleoproterozoic } \\
\text { (about } 2,000 \mathrm{Ma} \text { ) }\end{array}$ & $\begin{array}{l}\text { Belt interpreted as forming during the collision between } \\
\text { Tynda and West Aldan terranes in Aldan-Stanovoy } \\
\text { region and during subsequent collapse of orogenic belt. } \\
\text { Cause of collision was amalgamation of terranes during } \\
\text { the formation of the North Asia Craton. Au deposits } \\
\text { occur shear zones that cut metamorphosed mafic and } \\
\text { ultramafic and plutonic rock. }\end{array}$ \\
\hline
\end{tabular}




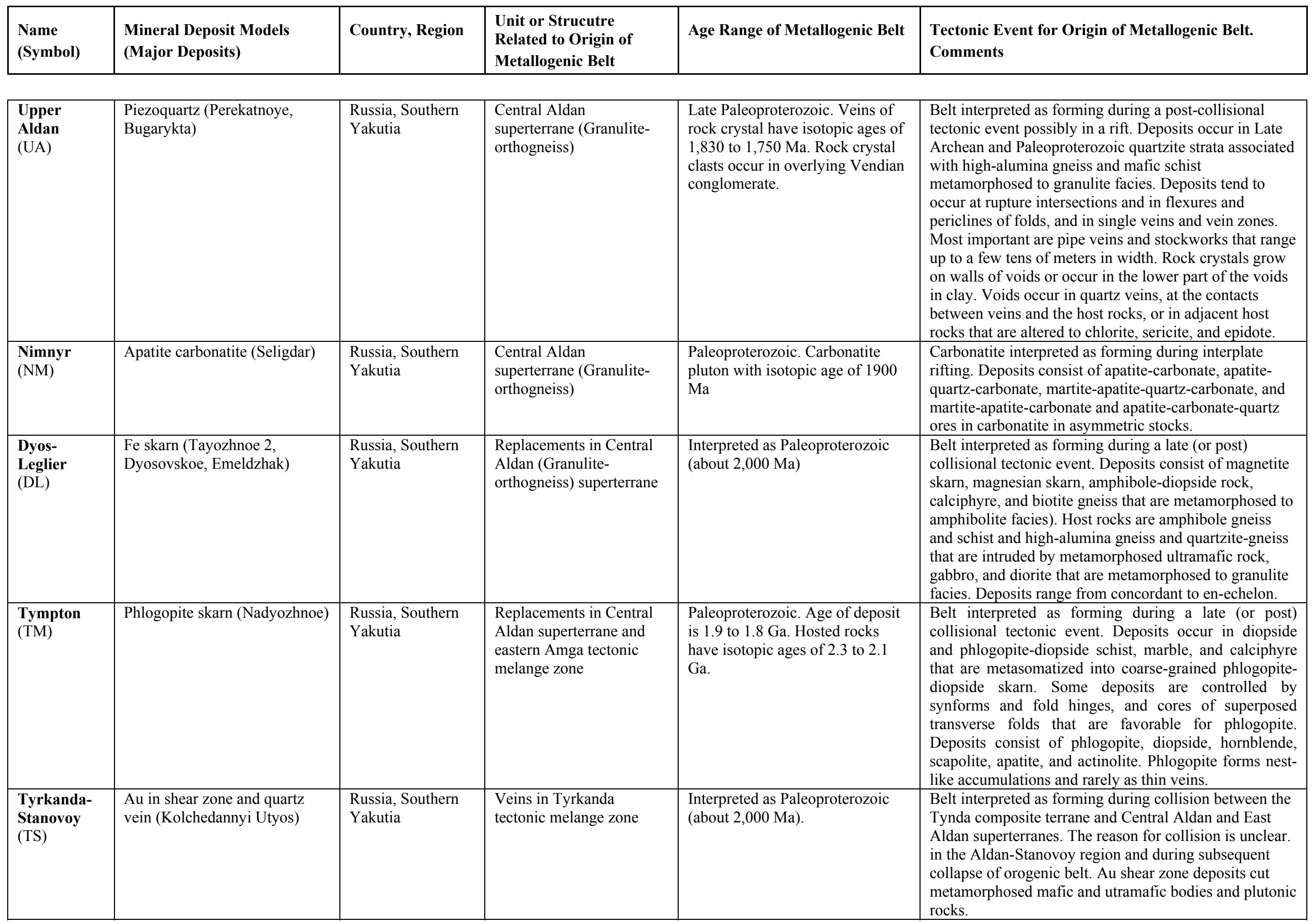




\begin{tabular}{|c|c|c|c|c|c|}
\hline $\begin{array}{l}\text { Name } \\
\text { (Symbol) }\end{array}$ & $\begin{array}{l}\text { Mineral Deposit Models } \\
\text { (Major Deposits) }\end{array}$ & Country, Region & $\begin{array}{l}\text { Unit or Strucutre } \\
\text { Related to Origin of } \\
\text { Metallogenic Belt }\end{array}$ & Age Range of Metallogenic Belt & $\begin{array}{l}\text { Tectonic Event for Origin of Metallogenic Belt. } \\
\text { Comments }\end{array}$ \\
\hline $\begin{array}{l}\text { Uchur } \\
\text { (UH) }\end{array}$ & Phlogopite skarn (Megyuskan) & $\begin{array}{l}\text { Russia, Southern } \\
\text { Yakutia }\end{array}$ & $\begin{array}{l}\text { Replacements in East } \\
\text { Aldan superterrane - } \\
\text { Batomga composite } \\
\text { terrane ) }\end{array}$ & $\begin{array}{l}\text { Interpreted as Paleoproterozoic } \\
\text { (about } 2,000 \mathrm{Ma} \text { ) }\end{array}$ & $\begin{array}{l}\text { Belt interpreted as forming during a late-stage or post- } \\
\text { collisional tectonic event as a result of collision between } \\
\text { the Central Aldan and East Aldan superterranes. The } \\
\text { reason for the collision is unclear. }\end{array}$ \\
\hline $\begin{array}{l}\text { Kavakta } \\
(\mathrm{KV})\end{array}$ & $\begin{array}{l}\text { Magmatic and metasomatic } \\
\text { apatite(?) (Kavakta) }\end{array}$ & $\begin{array}{l}\text { Russia, Southern } \\
\text { Yakutia }\end{array}$ & $\begin{array}{l}\text { Mafic-ultramafic plutons } \\
\text { in Amga tectonic melange } \\
\text { zone }\end{array}$ & Interpreted as Paleoproterozoic & $\begin{array}{l}\text { Belt interpreted forming during rifting related to } \\
\text { break up of a hypothetical Late Archean continent } \\
\text { at } 2.5 \text { to } 2.3 \mathrm{Ga} \text {. }\end{array}$ \\
\hline $\begin{array}{l}\text { Mugursk } \\
(\mathrm{MG})\end{array}$ & $\begin{array}{l}\text { Banded iron formation (BIF, } \\
\text { Algoma Fe) (Mugurskoye) }\end{array}$ & $\begin{array}{l}\text { Southeast Tuva, } \\
\text { Altai-Sayan folded } \\
\text { area, Russia }\end{array}$ & $\begin{array}{l}\text { Sangilen terrane (Passive } \\
\text { continental margin) }\end{array}$ & Paleoproterozoic & $\begin{array}{l}\text { Belt interpreted as forming in Tuva-Mongolian } \\
\text { microcontinent margin as a fragment of Laurasia. BIF } \\
\text { deposits occur in metamorphosed Paleoproterozoic } \\
\text { sedimentary rocks. }\end{array}$ \\
\hline $\begin{array}{l}\text { Khan Hohii } \\
(\mathrm{KH})\end{array}$ & $\begin{array}{l}\text { Banded iron formation (BIF, } \\
\text { Algoma Fe) (Tomorchuluut) }\end{array}$ & $\begin{array}{l}\text { Northwestern } \\
\text { Mongolia }\end{array}$ & $\begin{array}{l}\text { Baydrag terrane } \\
\text { (Cratonal), Khan Hohii } \\
\text { and North Songino } \\
\text { fragments }\end{array}$ & $\begin{array}{l}\text { Interpreted age if } \\
\text { Paleoproterozoic. } \mathrm{Pb}-\mathrm{Pb} \text { zircon } \\
\text { isotopic age of Songino gneiss is } \\
1,863 \mathrm{Ma}\end{array}$ & $\begin{array}{l}\text { BIF deposits hosted in Paleoproterozoic gneiss, } \\
\text { amphibolite, crystalline schist marble and quartzite } \\
\text { derived from a volcanic and clastic sedimentary rock } \\
\text { basin that is interpreted as forming in a continental } \\
\text { margin arc. }\end{array}$ \\
\hline $\begin{array}{l}\text { Tarvagatai } \\
\text { (TA) }\end{array}$ & $\begin{array}{l}\text { Banded iron formation (BIF, } \\
\text { Algoma Fe)(Salbart group); } \\
\text { Mafic-ultramafic related Ti-Fe } \\
( \pm \mathrm{V}) \text { (Salbart uul) }\end{array}$ & Central Mongolia & $\begin{array}{l}\text { Baydrag terrane } \\
\text { (Cratonal), Tarvagatai } \\
\text { fragment }\end{array}$ & $\begin{array}{l}\text { Paleoproterozoic(?). Host } \\
\text { metamorphic complex is intruded } \\
\text { by Most uul gabbro and } \\
\text { anorthosite complex with isotopic } \\
\text { ages of } 1,800 \text { to } 3,000 \mathrm{Ma} \text {. Zircon } \\
\text { isotopic ages for anorthosite range } \\
\text { from } 1800 \text { to } 3,000 \mathrm{Ma}\end{array}$ & $\begin{array}{l}\text { BIF occurrences are hosted in lower Proterozoic gneiss, } \\
\text { amphibolite, schist marble and quartzite derived from a } \\
\text { volcaniclastic and sedimentary rock deposited in a } \\
\text { volcaniclastic basin. Anorthosite hosting Ti-Fe } \\
\text { occurrences is interpreted as forming in a continental } \\
\text { margin arc. }\end{array}$ \\
\hline
\end{tabular}




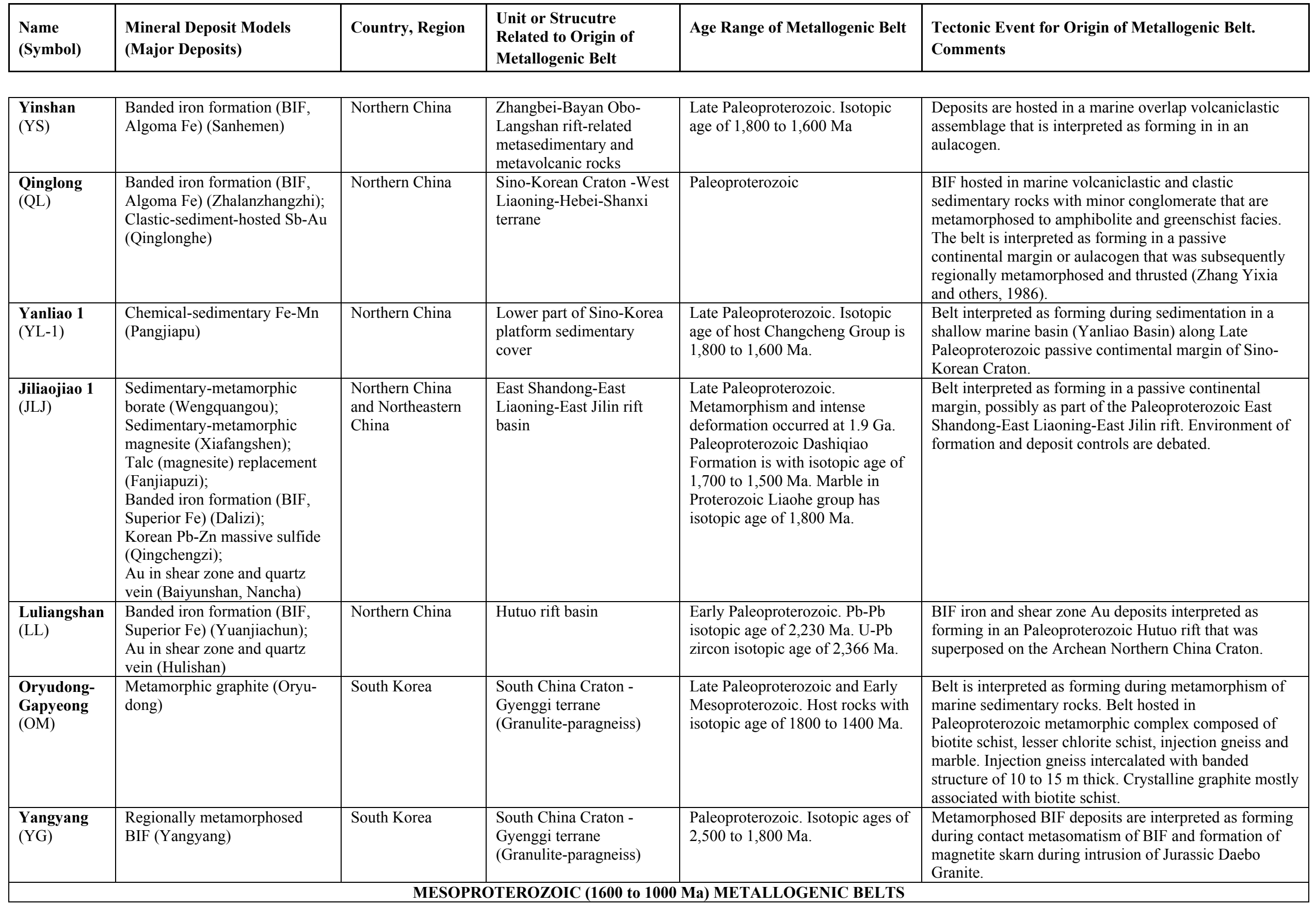




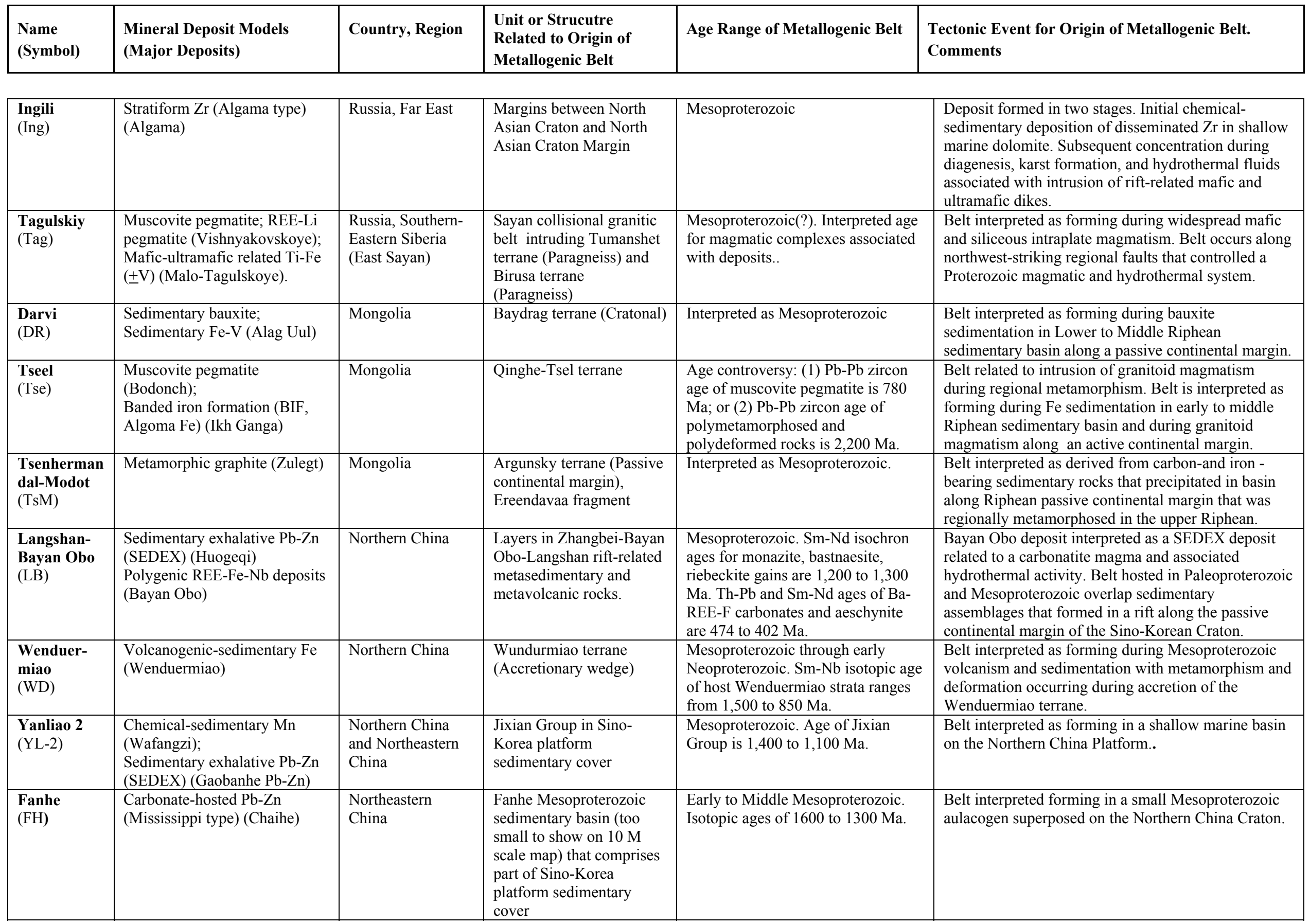




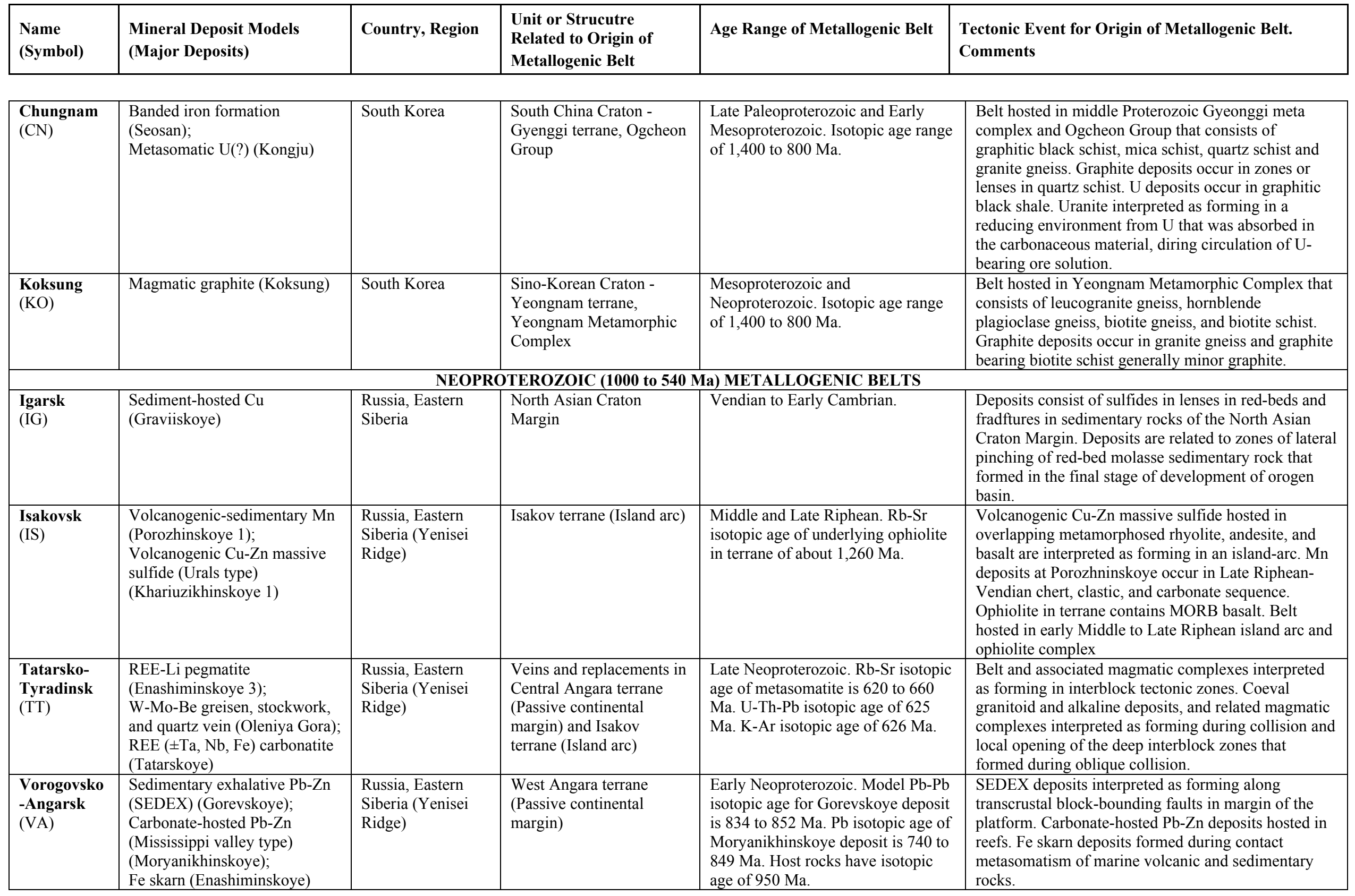




\begin{tabular}{|c|c|c|c|c|c|}
\hline $\begin{array}{l}\text { Name } \\
\text { (Symbol) }\end{array}$ & $\begin{array}{l}\text { Mineral Deposit Models } \\
\text { (Major Deposits) }\end{array}$ & Country, Region & $\begin{array}{l}\text { Unit or Strucutre } \\
\text { Related to Origin of } \\
\text { Metallogenic Belt }\end{array}$ & Age Range of Metallogenic Belt & $\begin{array}{l}\text { Tectonic Event for Origin of Metallogenic Belt. } \\
\text { Comments }\end{array}$ \\
\hline $\begin{array}{l}\text { Kyllakh } \\
(\mathrm{KY})\end{array}$ & $\begin{array}{l}\text { Carbonate-hosted } \mathrm{Pb}-\mathrm{Zn} \\
\text { (Mississippi valley type) } \\
\text { (Sardana, Urui, Pereval'noe) }\end{array}$ & Russia, Far East & $\begin{array}{l}\text { North Asian Craton } \\
\text { Margin - Verkhoyansk } \\
\text { fold and thrust belt }\end{array}$ & Vendian & $\begin{array}{l}\text { Belt interpreted as forming on passive margin of the } \\
\text { North Asian Craton in the Vendian. Economic } \\
\text { deposits occur in areas of facial thinning of dolomite. }\end{array}$ \\
\hline $\begin{array}{l}\text { Angara-Pit } \\
\text { (AP) }\end{array}$ & $\begin{array}{l}\text { Sedimentary siderite Fe } \\
\text { (Nizhne-Angarskoye); } \\
\text { Volcanogenic-sedimentary Fe } \\
\end{array}$ & $\begin{array}{l}\text { Russia, Eastern } \\
\text { Siberia (Yenisei } \\
\text { Ridge) } \\
\end{array}$ & $\begin{array}{l}\text { North Asian Craton } \\
\text { Margin (East Angara fold } \\
\text { and thrust belt) }\end{array}$ & Upper Riphean. & $\begin{array}{l}\text { Belt interpreted as forming during pre-orogenic } \\
\text { subsidence of the North Asian Craton margin in a } \\
\text { back-arc (interland) sedimentary basin. }\end{array}$ \\
\hline $\begin{array}{l}\text { Kansk } \\
(\mathrm{KN})\end{array}$ & $\begin{array}{l}\text { Au in shear zone and quartz } \\
\text { vein (Bogunai); } \\
\text { REE-Li pegmatite } \\
\text { (Barginskoye), } \\
\text { W-Mo-Be greisen, stockwork, } \\
\text { and quartz vein (Kanskoye) }\end{array}$ & $\begin{array}{l}\text { Southern Russia, } \\
\text { Yenisei Ridge }\end{array}$ & $\begin{array}{l}\text { Veins in Kan terrane } \\
\text { (Cratonal) }\end{array}$ & $\begin{array}{l}\text { Early Neoproterozoic . Pb isochron } \\
\text { is } 850 \pm 50 \mathrm{Ma} \text {; U-Th- } \mathrm{Pb} \text { isochron of } \\
920 \pm 50 \mathrm{Ma} \text {. }\end{array}$ & $\begin{array}{l}\text { Belt interpreted as forming during tectonic and } \\
\text { magmatic activation of the Angara-Kan block. Au } \\
\text { deposits related to small mafic intrusions that occur } \\
\text { along the Sayan-Yenisei deep fault zone. W-Mo } \\
\text { greisen and REE vein and pegmatite deposits of } \\
\text { presumed Late-Riphean age related to early-stage } \\
\text { collisional granitoids. }\end{array}$ \\
\hline $\begin{array}{l}\text { Tonodskiy } \\
\text { (Tnd) }\end{array}$ & $\begin{array}{l}\text { Au in black shale (Chertovo } \\
\text { Koryto) }\end{array}$ & $\begin{array}{l}\text { Russia, Northern } \\
\text { Transbaikalia }\end{array}$ & Tonod greenschist terrane & Riphean. & $\begin{array}{l}\text { Initial gold deposition from hydrothermal- } \\
\text { metamorphic processes that occurred during } \\
\text { Proterozoic regional metamorphism related to } \\
\text { accretion and generation of Chuya-Nechera } \\
\text { granitoids. Subsequent economic concentration } \\
\text { during late Riphean tectonism and magmatism. } \\
\text { Subsequent economic concentration during late } \\
\text { Riphean tectonism and magmatism with intrusion of } \\
\text { magmatic rocks along transform microplate } \\
\text { boundaries and within plate (plume) environment. }\end{array}$ \\
\hline $\begin{array}{l}\text { Baikalo- } \\
\text { Muiskiy } \\
\text { (BM) }\end{array}$ & $\begin{array}{l}\text { Volcanogenic-hydrothermal- } \\
\text { sedimentary massive sulfide } \\
\mathrm{Pb}-\mathrm{Zn}( \pm \mathrm{Cu}) ; \\
\text { Polymetallic }(\mathrm{Pb}, \mathrm{Zn}, \mathrm{Ag}) \\
\text { carbonate-hosted metasomatite; } \\
\text { Serpentinite-hosted asbestos } \\
\text { (Kholodninskoye, Lugovoye, } \\
\text { Molodezhnoye) }\end{array}$ & $\begin{array}{l}\text { Russia, Northern } \\
\text { Transbaikalia }\end{array}$ & $\begin{array}{l}\text { Baikal-Muya terrane } \\
\text { (Island arc) and Muya } \\
\text { terrane (Metamorphic) }\end{array}$ & Neoproterozoic. & $\begin{array}{l}\text { Various deposits in belt interpreted as forming in } \\
\text { Baikal-Muya island arc or during Riphean accretion } \\
\text { of terrane with Muya metamorphic terrane and } \\
\text { Olokit-Delunuran accretionary wedge terrane. }\end{array}$ \\
\hline
\end{tabular}




\begin{tabular}{|c|c|c|c|c|c|}
\hline $\begin{array}{l}\text { Name } \\
\text { (Symbol) }\end{array}$ & $\begin{array}{l}\text { Mineral Deposit Models } \\
\text { (Major Deposits) }\end{array}$ & Country, Region & $\begin{array}{l}\text { Unit or Strucutre } \\
\text { Related to Origin of } \\
\text { Metallogenic Belt }\end{array}$ & Age Range of Metallogenic Belt & $\begin{array}{l}\text { Tectonic Event for Origin of Metallogenic Belt. } \\
\text { Comments }\end{array}$ \\
\hline $\begin{array}{l}\text { Olokitskiy } \\
\text { (OL) }\end{array}$ & $\begin{array}{l}\text { Volcanogenic-hydrothermal- } \\
\text { sedimentary massive sulfide } \\
\mathrm{Pb}-\mathrm{Zn}( \pm \mathrm{Cu}) \text { (Kuroko, Altai } \\
\text { types) (Kholodninskoye) }\end{array}$ & $\begin{array}{l}\text { Russia, Northern } \\
\text { Transbaikalia }\end{array}$ & $\begin{array}{l}\text { Olokit-Delunuran terrane } \\
\text { (Accretionary wedge) }\end{array}$ & $\begin{array}{l}\text { Neoproterozoic. Isotopic ages of } \\
\text { about } 1,000 \text { to } 740 \mathrm{Ma} \text {. }\end{array}$ & $\begin{array}{l}\text { Belt is interpreted as forming in island arc or back arc } \\
\text { sequence incorporated into an accretionary wedge. }\end{array}$ \\
\hline $\begin{array}{l}\text { Mrass } \\
(\mathrm{MR})\end{array}$ & $\begin{array}{l}\text { Sedimentary phosphate } \\
\text { (Tamalykskoye) }\end{array}$ & $\begin{array}{l}\text { Russia, Southern- } \\
\text { Eastern Siberia } \\
\text { (Kuznetsk Alatau } \\
\text { Mountains) }\end{array}$ & $\begin{array}{l}\text { Altai-Sayan back-arc } \\
\text { basin (Mrassu-Bateni unit) }\end{array}$ & Vendian to Early Cambrian & $\begin{array}{l}\text { Belt interpreted as forming during shallow-water } \\
\text { marine sedimentation in a back-arc environment. }\end{array}$ \\
\hline $\begin{array}{l}\text { Lysansk } \\
\text { (LS) }\end{array}$ & $\begin{array}{l}\text { Mafic-ultramafic related Ti-Fe } \\
( \pm \mathrm{V}) \text { (Lysanskoye) }\end{array}$ & $\begin{array}{l}\text { Russia, Southern- } \\
\text { Eastern Siberia } \\
\text { (East Sayan) }\end{array}$ & $\begin{array}{l}\text { Mafic-ultramafic plutons } \\
\text { in Kuvai terrane } \\
\text { (Accretionary wedge) } \\
\end{array}$ & Middle to Late Riphean. & $\begin{array}{l}\text { Belt interpreted as forming in a Middle to Late } \\
\text { Riphean ensialic island arc that was incorporated into } \\
\text { an accretionary wedge. }\end{array}$ \\
\hline $\begin{array}{l}\text { Prisayan- } \\
\text { skiy } \\
(\operatorname{PrS})\end{array}$ & $\begin{array}{l}\text { REE ( } \pm \mathrm{Ta}, \mathrm{Nb}, \mathrm{Fe}) \text { carbonatite; } \\
\text { Mafic-ultramafic related Ti-Fe } \\
( \pm \mathrm{V}) \text { (Beloziminskoye) } \\
\text { Diamond-bearing kimberlite } \\
\text { (Onotskoe) } \\
\text { Talc (magnesite) replacement } \\
\text { (Ingashinskoye) }\end{array}$ & $\begin{array}{l}\text { Russia, Southern- } \\
\text { Eastern Siberia } \\
\text { (East Sayan) }\end{array}$ & $\begin{array}{l}\text { Various units associated } \\
\text { with the Onot granite- } \\
\text { greenstone and } \\
\text { Sharizhalgay tonalite- } \\
\text { trondhjemite gneiss } \\
\text { terranes: (1) mafic- } \\
\text { ultramafic plutons in the } \\
\text { Ziminsky complex; (2) } \\
\text { upper part of Onot terrane } \\
\text { that consists of } \\
\text { interbedded amphibolite, } \\
\text { and magnesite and talc } \\
\text { layers; and (3) ultramafic } \\
\text { alkaline plutonic rocks } \\
\text { that intrude; and (4) sparse } \\
\text { micaceous kimberlite } \\
\text { dikes. Various host units } \\
\text { are too small to show at } 10 \\
\text { M scale. }\end{array}$ & $\begin{array}{l}\text { Late Neoproterozoic. } \mathrm{Rb}-\mathrm{Sr} \\
\text { isochron age for talc deposit is } 633 \\
\mathrm{Ma}\end{array}$ & $\begin{array}{l}\text { Belt occurs in enderbite-gneiss, tonalite-trondjemite, } \\
\text { anorthosite-paragneiss formation of terranes that are } \\
\text { fragments of Precambrian craton crystalline } \\
\text { basement. Host terranes are uplifted parts of North } \\
\text { Asian Craton. }\end{array}$ \\
\hline
\end{tabular}




\begin{tabular}{|c|c|c|c|c|c|}
\hline $\begin{array}{l}\text { Name } \\
\text { (Symbol) }\end{array}$ & $\begin{array}{l}\text { Mineral Deposit Models } \\
\text { (Major Deposits) }\end{array}$ & Country, Region & $\begin{array}{l}\text { Unit or Strucutre } \\
\text { Related to Origin of } \\
\text { Metallogenic Belt }\end{array}$ & Age Range of Metallogenic Belt & $\begin{array}{l}\text { Tectonic Event for Origin of Metallogenic Belt. } \\
\text { Comments }\end{array}$ \\
\hline $\begin{array}{l}\text { Bokson- } \\
\text { Kitoiskiy } \\
\text { (B-K) }\end{array}$ & $\begin{array}{l}\text { Sedimentary bauxite } \\
\text { (Boksonskoye); } \\
\text { Magmatic nepheline } \\
\text { (Botogolskoye); } \\
\text { Serpentine-hosted asbestos; } \\
\text { Au in shear zone and quartz } \\
\text { vein (Zun-Kholba) }\end{array}$ & $\begin{array}{l}\text { Russia, Southern- } \\
\text { Eastern Siberia } \\
\text { (East Sayan) }\end{array}$ & $\begin{array}{l}\text { Layers in, and veins and } \\
\text { plutons intruding or } \\
\text { associated with Belaya- } \\
\text { Kitoy metamorphic } \\
\text { terrane, Hug accretionary } \\
\text { wedge terrane, Tunka } \\
\text { tonalite-trondhjemite- } \\
\text { gneiss terrane, Tannuola } \\
\text { plutonic belt, and } \\
\text { Huvsgol-Bokson } \\
\text { sedimentary overlap } \\
\text { assemblage. }\end{array}$ & $\begin{array}{l}\text { Neoproterozoic through Silurian. } \\
\text { Neoproterozoic sedimentary rocks } \\
\text { with and Cambrian through Silurian } \\
\text { metamorphism, hydrothermal } \\
\text { alternation, and plutonic intrusion. } \\
\text { Younger suture complex of } \\
\text { Sumsunur tonalite complex with U- } \\
\mathrm{Pb} \text { and } \mathrm{Rb}-\mathrm{Sr} \text { isotopics ages of } 790 \\
\mathrm{Ma} \text {. }\end{array}$ & $\begin{array}{l}\text { Belt hosted in metamorphic, oceanic, accretionary } \\
\text { wedge, and accretionary wedge, and tonalite- } \\
\text { trondhjemite-gneiss terranes that underwent Cambrian } \\
\text { through Silurian metamorphism, hydrothermal } \\
\text { alternation, and plutonic intrusion. Deposits formed in } \\
\text { multiple events. }\end{array}$ \\
\hline $\begin{array}{l}\text { Lake } \\
\text { (LA) }\end{array}$ & $\begin{array}{l}\text { Volcanogenic Cu-Zn massive } \\
\text { sulfide (Urals type) (Borts uul); } \\
\text { Volcanogenic-sedimentary Fe; } \\
\text { Podiform } \mathrm{Cr} \text {; Mafic-ultramafic } \\
\text { related Ti-Fe }( \pm \mathrm{V}) ; \\
\mathrm{Cu}( \pm \mathrm{Fe}, \mathrm{Au}, \mathrm{Ag}, \mathrm{Mo}) \text { skarn; } \\
\mathrm{Fe} \text { skarn; } \\
\text { Granitoid-related Au vein } \\
\text { (Khyargas) } \\
\text { Cyprus Cu-Zn Massive Sulfide } \\
\text { (Naran Davaa); } \\
\text { Mafic-ultramafic related Cu- } \\
\text { Ni-PGE (Tsagdaltyn Davaa) }\end{array}$ & Western Mongolia & Lake terrane (Island arc) & $\begin{array}{l}\text { Late Neoproterozoic. Khantayshir } \\
\text { ophiolite with } \mathrm{U}-\mathrm{Pb} \text { zircon isotopic } \\
\text { age of } 568 \pm 4 \mathrm{Ma} \text {. Dariv ophiolite } \\
\text { with } \mathrm{U}-\mathrm{Pb} \text { zircon isotopic age of } 573 \\
6 \mathrm{Ma} \text {. }\end{array}$ & $\begin{array}{l}\text { Various deposits in belt are interpreted as forming } \\
\text { during sea floor spreading volcanism and related } \\
\text { mafic-ultramafic magmatism, and in subduction- } \\
\text { related island arc volcanism and mafic plutonism, and } \\
\text { and multiple-phase granitic magmatism. }\end{array}$ \\
\hline $\begin{array}{l}\text { Tsagaa- } \\
\text { nolom } \\
(\mathrm{TO})\end{array}$ & $\begin{array}{l}\text { Sedimentary phosphate (Zuun } \\
\text { Arts); } \\
\text { Volcanogenic-sedimentary Mn } \\
\text { (Khagnuur) }\end{array}$ & Central Mongolia & $\begin{array}{l}\text { Huvsgol-Bokson } \\
\text { sedimentary overlap } \\
\text { assemblage }\end{array}$ & Vendian through Early Cambrian. & $\begin{array}{l}\text { Belt interpreted as forming during sedimentation in } \\
\text { carbonate-dominated basin along a continental shelf. }\end{array}$ \\
\hline
\end{tabular}




\begin{tabular}{|c|c|c|c|c|c|}
\hline $\begin{array}{l}\text { Name } \\
\text { (Symbol) }\end{array}$ & $\begin{array}{l}\text { Mineral Deposit Models } \\
\text { (Major Deposits) }\end{array}$ & Country, Region & $\begin{array}{l}\text { Unit or Strucutre } \\
\text { Related to Origin of } \\
\text { Metallogenic Belt }\end{array}$ & Age Range of Metallogenic Belt & $\begin{array}{l}\text { Tectonic Event for Origin of Metallogenic Belt. } \\
\text { Comments }\end{array}$ \\
\hline $\begin{array}{l}\text { Jixi } \\
(\mathrm{JX})\end{array}$ & $\begin{array}{l}\text { Banded iron formation (BIF, } \\
\text { Algoma Fe) (Shuangyashan); } \\
\text { Homestake Au } \\
\text { (Dongfengshan); } \\
\text { Metamorphic graphite; } \\
\text { (Liumao); } \\
\text { Metamorphic sillimanite }\end{array}$ & $\begin{array}{l}\text { Northeastern } \\
\text { China }\end{array}$ & $\begin{array}{l}\text { Jiamusi terrane } \\
\text { (Metamorphic) terrane and } \\
\text { Zhangguangcailing } \\
\text { (Continental margin arc) } \\
\text { superterrane }\end{array}$ & Neoproterozoic to Cambrian. & $\begin{array}{l}\text { Belt part of a khondalite that is interpreted as derived } \\
\text { from Al-rich mudstone and carbonates of the Mashan } \\
\text { and the Xingdong groups that were deposited in a } \\
\text { shallow sea and isolated oceanic basin and lagoon. }\end{array}$ \\
\hline $\begin{array}{l}\text { Damiao } \\
(\mathrm{DM})\end{array}$ & $\begin{array}{l}\text { Mafic-ultramafic related Ti-Fe } \\
( \pm \text { V) (Damiao); } \\
\text { Zoned mafic-ultramafic Cr- } \\
\text { PGE (Gaositai) }\end{array}$ & Northern China & $\begin{array}{l}\text { Mafic-ultramafic plutons } \\
\text { intruding Sino-Korean } \\
\text { Craton - West Liaoning- } \\
\text { Hebei-Shanxi terrane } \\
\text { (Granulite-orthogneiss) }\end{array}$ & $\begin{array}{l}\text { Neoproterozoic. K-Ar age of the } \\
\text { deposit-related anorthosite is } 604 \text { to } \\
992 \mathrm{Ma} \text {. }\end{array}$ & $\begin{array}{l}\text { Belt hosted in Neoproterozoic mafic-ultramafic } \\
\text { plutons that intrude Archean gneiss that intrude } \\
\text { Archean crystalline rocks of West Liaoning-Hebei- } \\
\text { Shanxi terrane. The plutons occur along northwest- } \\
\text { trending major deep faults along the northern margin } \\
\text { of the Sino-Korean Platform. The mafic and } \\
\text { ultramafic intrusions have isotopic ages of } 604.4 \text { to } \\
992 \mathrm{Ma} \text {. The plutons and deposits are interpreted as } \\
\text { forming during interplate magmatism related to an } \\
\text { Neoproterozoic active continental margin along the } \\
\text { north margin of the Sino-Korean Craton. }\end{array}$ \\
\hline \multicolumn{6}{|c|}{ CAMBRIAN THROUGH SILURIAN (540 to 410 Ma) METALLOGENIC BELTS } \\
\hline $\begin{array}{l}\text { Tuora-Sis } \\
\text { (Tuo) }\end{array}$ & $\begin{array}{l}\text { Carbonate-hosted } \mathrm{Pb}-\mathrm{Zn} \\
\text { (Mississippi valley type) } \\
\text { (Mengeniler) }\end{array}$ & Russia,Yakutia & $\begin{array}{l}\text { North Asian Craton } \\
\text { Margin - Verkhoyansk } \\
\text { fold and thrust belt } \\
\text { (Passive continental } \\
\text { margin) }\end{array}$ & Early Cambrian. & $\begin{array}{l}\text { Belt interpreted as forming during sedimentation after } \\
\text { Neoproterozoic rifting along the passive continental } \\
\text { margin of North Asian Craton. Economic deposits } \\
\text { occur in areas of facial thinning of dolomite. }\end{array}$ \\
\hline $\begin{array}{l}\text { Bedobinsk } \\
\text { (BED) }\end{array}$ & $\begin{array}{l}\text { Sediment-hosted } \mathrm{Cu} \\
\text { (Bedobinsk, Kurishskoye) }\end{array}$ & $\begin{array}{l}\text { Russia, Eastern } \\
\text { Siberia (Yenisey } \\
\text { Ridge area) }\end{array}$ & North Asian Craton & Middle to Late Cambrian. & $\begin{array}{l}\text { Belt interpreted as forming in an inland-sea basin } \\
\text { during post-saline stage of rock deposition. Main } \\
\text { source of copper were weathered Riphean rocks as } \\
\text { well as lode deposits in the Yenisei Ridge, and from } \\
\text { hydrothermal activity along deep-fault zones related } \\
\text { to rifting. }\end{array}$ \\
\hline
\end{tabular}




\begin{tabular}{|c|c|c|c|c|c|}
\hline $\begin{array}{l}\text { Name } \\
\text { (Symbol) }\end{array}$ & $\begin{array}{l}\text { Mineral Deposit Models } \\
\text { (Major Deposits) }\end{array}$ & Country, Region & $\begin{array}{l}\text { Unit or Strucutre } \\
\text { Related to Origin of } \\
\text { Metallogenic Belt }\end{array}$ & Age Range of Metallogenic Belt & $\begin{array}{l}\text { Tectonic Event for Origin of Metallogenic Belt. } \\
\text { Comments }\end{array}$ \\
\hline $\begin{array}{l}\text { Kiyalykh- } \\
\text { Uzen } \\
\text { (Kiy) }\end{array}$ & $\begin{array}{l}\mathrm{Cu}( \pm \mathrm{Fe}, \mathrm{Au}, \mathrm{Ag}, \mathrm{Mo}) \text { skarn } \\
\text { (Kiyalykh-Uzen, Juliya } \\
\text { Mednaya); } \\
\mathrm{W} \pm \mathrm{Mo} \pm \mathrm{Be} \text { skarn (Tuim); } \\
\text { Fe skarn (Samson); } \\
\text { W-Mo-Be greisen, stockwork, } \\
\text { and quartz vein (Verhne- } \\
\text { Askizskoye, Turtek) }\end{array}$ & $\begin{array}{l}\text { Russia, Southern- } \\
\text { Eastern Siberia } \\
\text { (Kuznetsk Alatau) }\end{array}$ & $\begin{array}{l}\text { Replacements related to } \\
\text { Tannuola plutonic belt } \\
\text { located in Altai-Sayan } \\
\text { back-arc basin (Mrassu- } \\
\text { Bateni unit) }\end{array}$ & $\begin{array}{l}\text { Early Ordovician to Early Silurian. } \\
{ }^{40} \mathrm{Ar}{ }^{39} \mathrm{Ar} \text { host-rock isotopic age of } \\
480 \text { to } 420 \mathrm{Ma}\end{array}$ & $\begin{array}{l}\text { Belt related to early Paleozoic collisional granitoids } \\
\text { that intrude Vendian and Cambrian shelf carbonate } \\
\text { and clastic-carbonate rocks during dextral-slip } \\
\text { movement along the Kuznetsk Alatau fault. }\end{array}$ \\
\hline $\begin{array}{l}\text { Kizir-Kazyr } \\
(\mathrm{KK})\end{array}$ & $\begin{array}{l}\text { Fe skarn (Irbinskoye); } \\
\text { Volcanogenic-sedimentary Fe } \\
\text { (Belokitatskoye); } \\
\text { Granitoid-related Au vein } \\
\text { (Olkhovskoye) }\end{array}$ & $\begin{array}{l}\text { Russia, Southern- } \\
\text { Eastern Siberia } \\
\text { (Eastern Sayan } \\
\text { Ridge) }\end{array}$ & $\begin{array}{l}\text { Replacements related to } \\
\text { Tannuola plutonic belt } \\
\text { (too small to show at } 10 \mathrm{M} \\
\text { scale) }\end{array}$ & $\begin{array}{l}\text { Middle Silurian. K-Ar isotopic age } \\
\text { for deposit-related gabbro, diorite, } \\
\text { and granodiorite plutons Irbinskoye } \\
\text { district is } 430 \mathrm{Ma}\end{array}$ & $\begin{array}{l}\text { Deposits hosted in gabbro, diorite, and granodiorite in } \\
\text { the collisional Tannuola plutonic belt, and in } \\
\text { volcanogenic-sedimentary rocks of the Kizir-Kazir } \\
\text { island-arc terrane. }\end{array}$ \\
\hline $\begin{array}{l}\text { North- } \\
\text { Sayanian } \\
\text { (NS) }\end{array}$ & $\begin{array}{l}\text { Fe skarn (Abakanskoye, } \\
\text { Anzass); } \\
\text { Cyprus Cu-Zn massive sulfide } \\
\text { (Mainskoye) }\end{array}$ & $\begin{array}{l}\text { Russia, Southern- } \\
\text { Eastern Siberia } \\
\text { (West Sayan } \\
\text { Mountains) }\end{array}$ & $\begin{array}{l}\text { Replacements related to } \\
\text { North Sayan terrane } \\
\text { (Island arc) }\end{array}$ & Early to Middle Cambrian. & $\begin{array}{l}\text { Belt interpreted as forming in volcanic basins in an } \\
\text { island-arc. Major faults played a significant role and } \\
\text { controlled sedimentary, volcanic, and intrusive } \\
\text { processes as well as the general linear structure of the } \\
\text { belt. }\end{array}$ \\
\hline $\begin{array}{l}\text { Khemchik- } \\
\text { Kurtushi- } \\
\text { binsk } \\
(\mathrm{KhK}) \\
\end{array}$ & $\begin{array}{l}\text { Serpentinite-hosted asbestos } \\
\text { (Actovrak, Sayanskoye) }\end{array}$ & $\begin{array}{l}\text { Russia, Southern- } \\
\text { Eastern Siberia } \\
\text { (Tuva area) }\end{array}$ & $\begin{array}{l}\text { Replacements related to } \\
\text { Kurtushiba terrane } \\
\text { (Accretionary wedge) }\end{array}$ & Vendian to Early Cambrian. & $\begin{array}{l}\text { Belt interpreted as forming during accretion of } \\
\text { Kurtushiba ophiolite belt along the major Tuva- } \\
\text { Sayanian fault in the Kurtushiba accretionary wedge } \\
\text { terrane that contains mainly oceanic rocks. }\end{array}$ \\
\hline $\begin{array}{l}\text { Ondumsk } \\
(\mathrm{ON})\end{array}$ & $\begin{array}{l}\text { Au skarn (Tardan); } \\
\text { Granitoid-related Au vein } \\
\text { (Proezdnoye) }\end{array}$ & $\begin{array}{l}\text { Russia, Southern- } \\
\text { Eastern Siberia } \\
\text { (Tuva area) }\end{array}$ & $\begin{array}{l}\text { Replacements related to } \\
\text { Tannuola plutonic belt }\end{array}$ & Late Cambrian to Ordovician & $\begin{array}{l}\text { Belt hosted in granitoid intrusions of the collisional } \\
\text { Tannuola complex that intrudes Early Cambrian } \\
\text { carbonate and volcanic rocks that are part of the } \\
\text { Ondum ensialic island arc terrane. }\end{array}$ \\
\hline
\end{tabular}




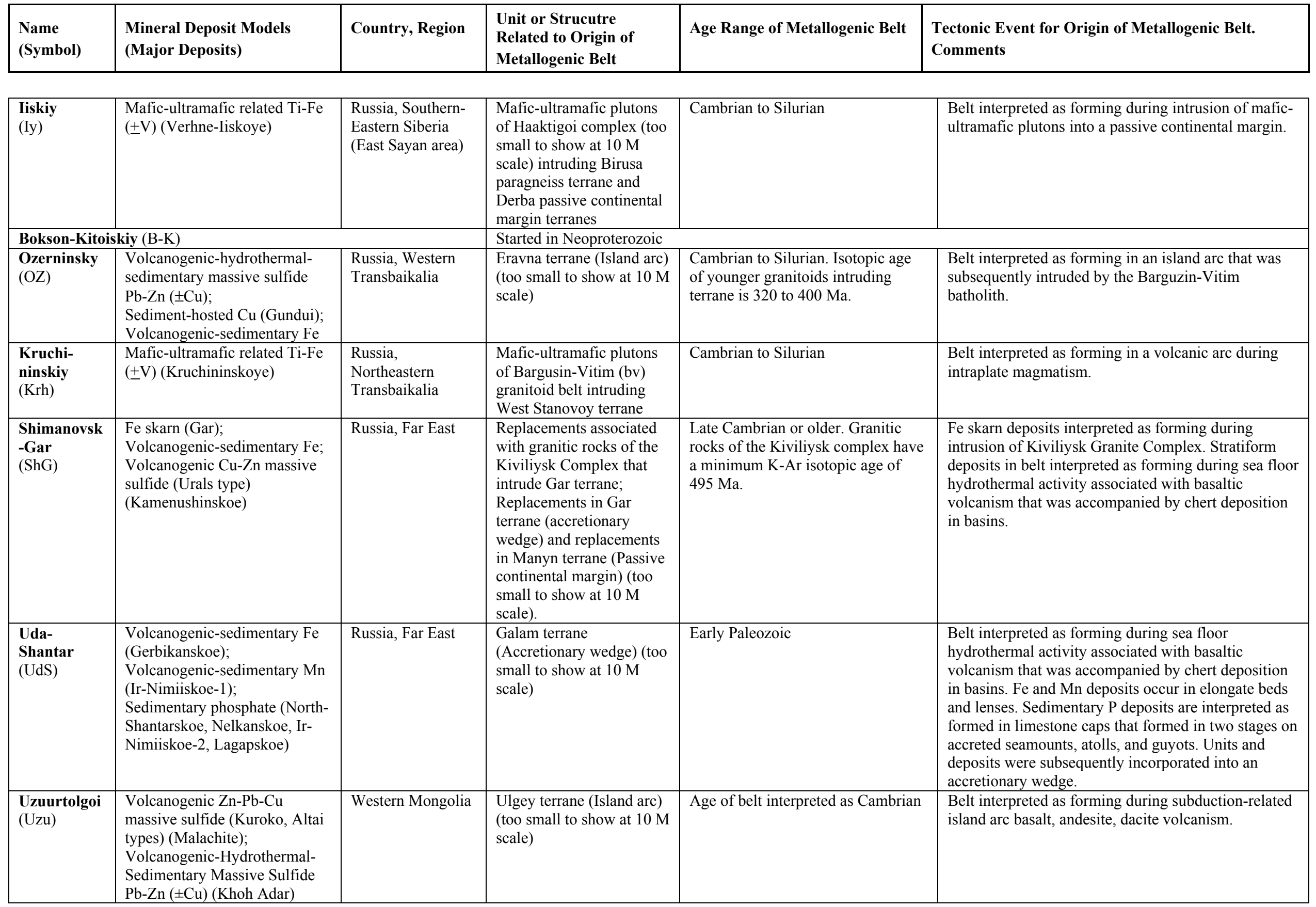




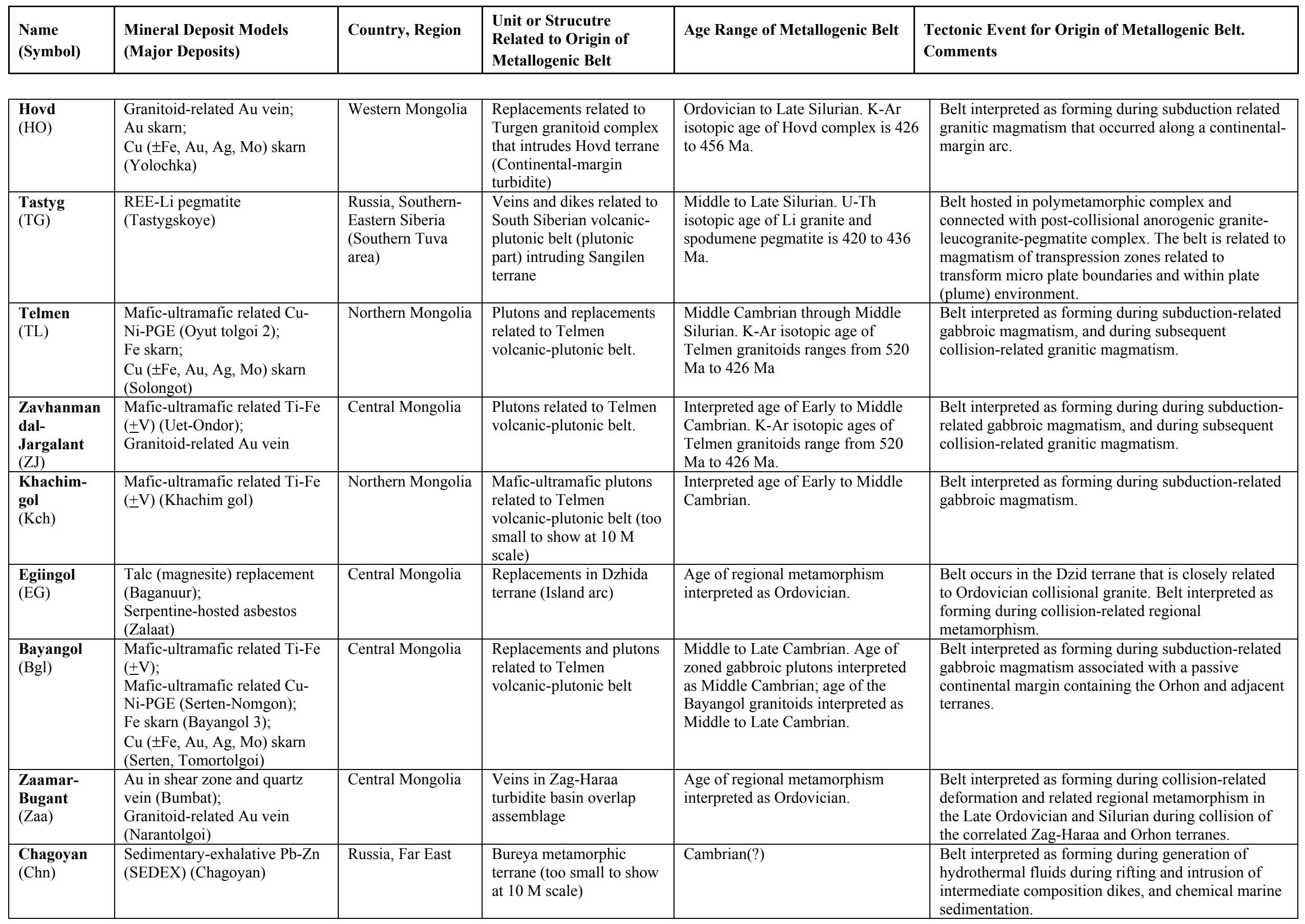




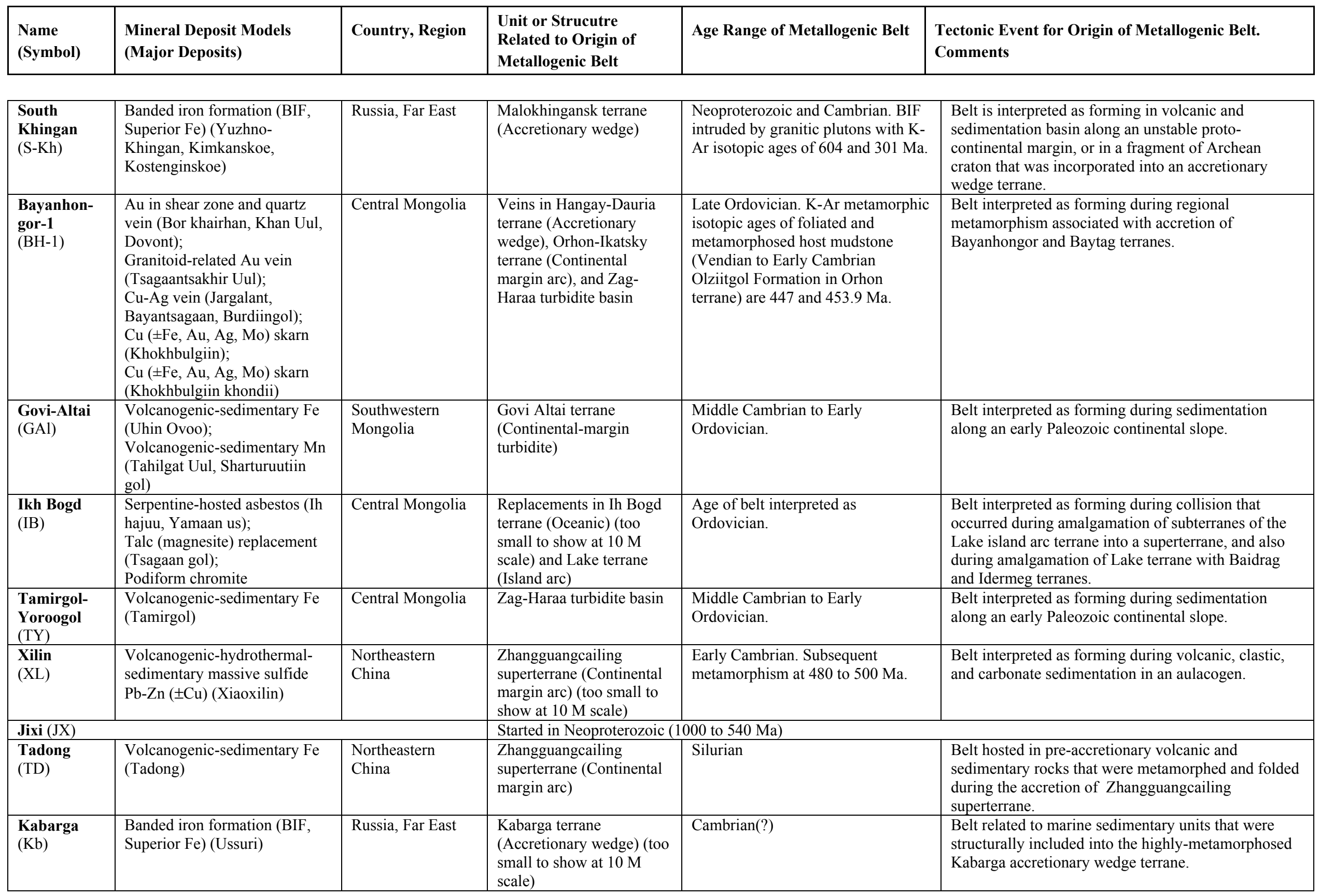




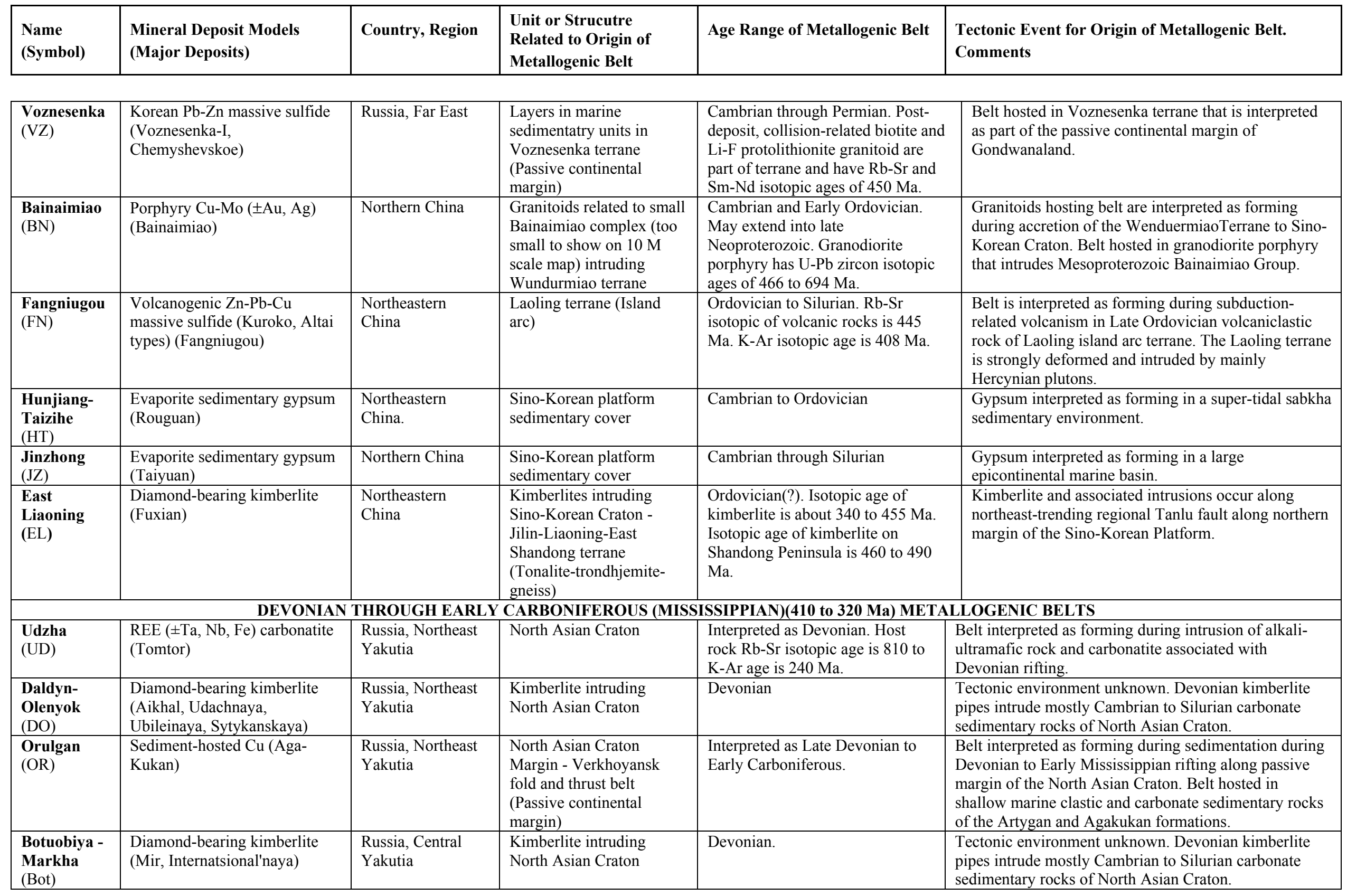




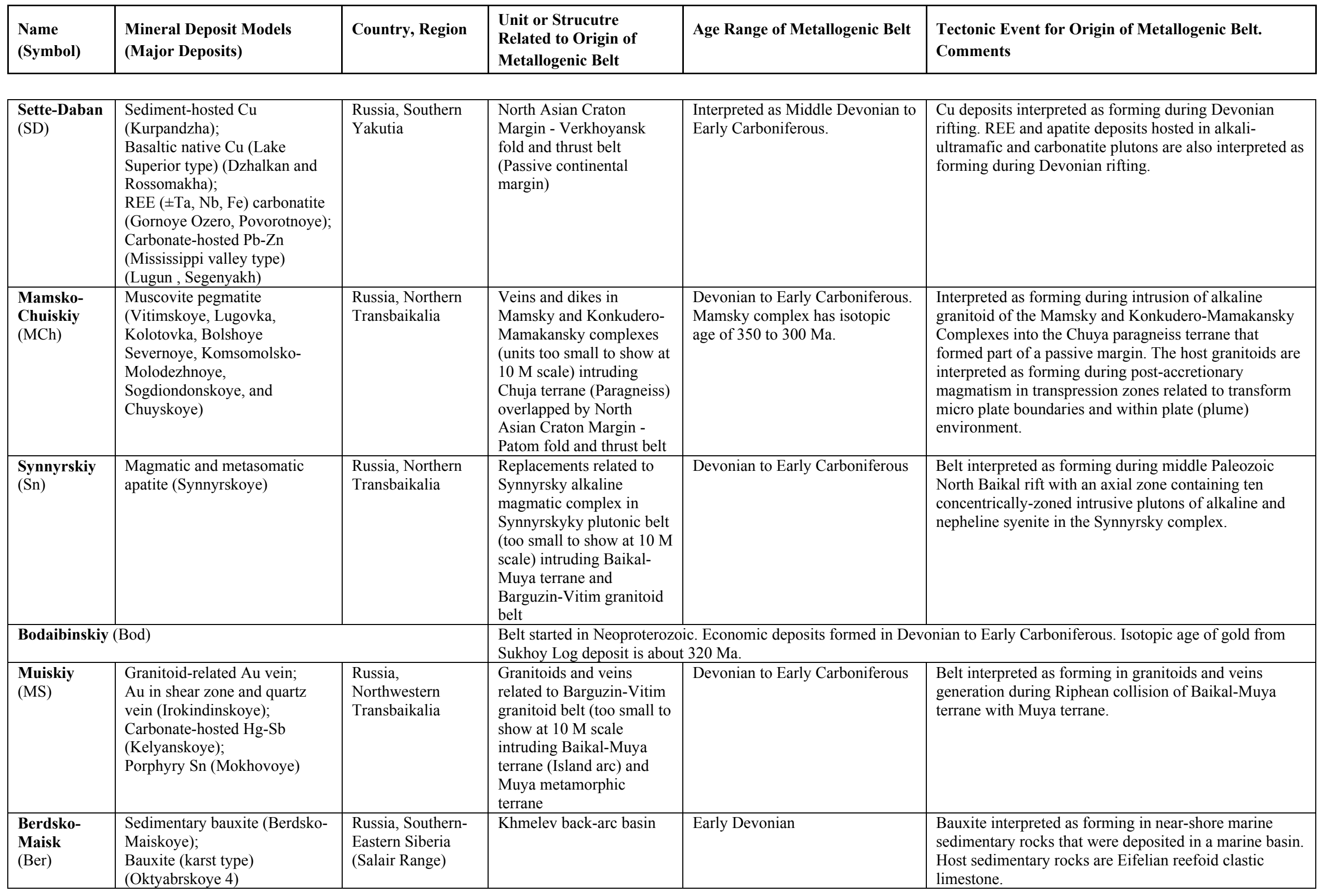




\begin{tabular}{|c|c|c|c|c|c|}
\hline $\begin{array}{l}\text { Name } \\
\text { (Symbol) }\end{array}$ & $\begin{array}{l}\text { Mineral Deposit Models } \\
\text { (Major Deposits) }\end{array}$ & Country, Region & $\begin{array}{l}\text { Unit or Strucutre } \\
\text { Related to Origin of } \\
\text { Metallogenic Belt }\end{array}$ & Age Range of Metallogenic Belt & $\begin{array}{l}\text { Tectonic Event for Origin of Metallogenic Belt. } \\
\text { Comments }\end{array}$ \\
\hline $\begin{array}{l}\text { Kiya- } \\
\text { Shaltyr } \\
\text { (Ksh) }\end{array}$ & $\begin{array}{l}\text { Magmatic nepheline (Kiya- } \\
\text { Shaltyr) }\end{array}$ & $\begin{array}{l}\text { Russia, Southern- } \\
\text { Eastern Siberia } \\
\text { (Kuznetsk Alatau } \\
\text { Mountains) }\end{array}$ & $\begin{array}{l}\text { Deposits related to } \\
\text { intrusions of South } \\
\text { Siberan volcanic-plutonic } \\
\text { belt }\end{array}$ & $\begin{array}{l}\text { Middle Devonian. Rb-Sr isochron } \\
\text { age of } 383 \mathrm{Ma}\end{array}$ & $\begin{array}{l}\text { Belt interpreted as forming during rift-related } \\
\text { magmatism above a hot spot. A }{ }^{87} \mathrm{Sr} /{ }^{86} \mathrm{Sr} \text { ratio of } 0.7053 \\
\text { for gabbros and urtite of the Kiya-Shaltyr pluton } \\
\text { indicates a deep-mantle origin. Nepheline plutons occur } \\
\text { along major fault zones. }\end{array}$ \\
\hline $\begin{array}{l}\text { Sorsk } \\
(\mathrm{SO})\end{array}$ & $\begin{array}{l}\text { Porphyry Mo }( \pm \mathrm{W}, \mathrm{Bi}) \\
\text { (Sorskoye); } \\
\text { Polymetallic }(\mathrm{Pb}, \mathrm{Zn}, \mathrm{Ag}) \\
\text { carbonate-hosted metasomatite } \\
\text { (Karasuk); } \\
\mathrm{Zn}-\mathrm{Pb}( \pm \mathrm{Ag}, \mathrm{Cu}) \text { skarn (Julia } \\
\text { Svintsovaya) }\end{array}$ & $\begin{array}{l}\text { Russia, Southern- } \\
\text { Eastern Siberia } \\
\text { (Kuznetsk Alatau } \\
\text { Mountains) }\end{array}$ & $\begin{array}{l}\text { Granitoids and associated } \\
\text { replacements related to } \\
\text { South Siberian volcanic- } \\
\text { plutonic belt. }\end{array}$ & $\begin{array}{l}\text { Early and Middle Devonian. }{ }^{40} \mathrm{Ar}- \\
{ }^{39} \mathrm{Ar} \text { isotopic age of deposits is } \\
385 \text { to } 400 \mathrm{Ma} \text {. K-feldspar and } \\
\text { albite metasomatite age is } 400 \text { to } \\
380 \mathrm{Ma} \text {. Host volcanic rocks with } \\
\mathrm{K}-\mathrm{Ar} \text { age of } 396 \mathrm{Ma} \text { and } \mathrm{Rb}-\mathrm{Sr} \\
\text { age of } 416 \mathrm{Ma} \text {. }\end{array}$ & $\begin{array}{l}\text { Belt interpreted as forming during Devonian subalkalic } \\
\text { porphyry magmatism related to interplate rifting. } \\
\text { Deposit-related porphyry intrusions intrude older early } \\
\text { Paleozoic granitoid plutons. Skarn and metasomatic } \\
\text { polymetallic deposits hosted in Vendian and Cambrian } \\
\text { shallow-water marine carbonate rocks. }\end{array}$ \\
\hline $\begin{array}{l}\text { Teisk } \\
\text { (TE) }\end{array}$ & $\begin{array}{l}\text { Fe skarn (Teiskoye, } \\
\text { Khaileolovskoye); } \\
\text { Mafic-ultramafic related Ti-Fe } \\
( \pm \mathrm{V}) \text { (Patynskoye, Kul-Taiga); } \\
\text { Volcanogenic-Sedimentary Fe } \\
\text { (Chilanskoye) }\end{array}$ & $\begin{array}{l}\text { Russia, Southern- } \\
\text { Eastern Siberia } \\
\text { (Kuznetsk Alatau } \\
\text { Mountains) }\end{array}$ & $\begin{array}{l}\text { Deposits related to } \\
\text { plutonic rocks of South } \\
\text { Siberian volcanic-plutonic } \\
\text { belt }\end{array}$ & $\begin{array}{l}\text { Early Devonian. K-Ar isotopic } \\
\text { ages for syenite-diorite of Malaya } \\
\text { Kul-Taiga pluton are } 411 \text { and } 438 \\
\text { Ma. K-Ar isotopic age of } \\
\text { Devonian volcanic rocks is } 396 \\
\text { Ma and Rb-Sr isotopic age is } 416 \\
\text { Ma. }\end{array}$ & $\begin{array}{l}\text { Belt interpreted as forming during interplate rifting that } \\
\text { formed South Minusa volcanic basin. Deposit-related } \\
\text { Early Devonian granosyenite plutons occur along } \\
\text { marginal faults of Devonian basins. }\end{array}$ \\
\hline $\begin{array}{l}\text { Chapsordag } \\
(\mathrm{ChD})\end{array}$ & $\begin{array}{l}\text { Barite vein (Chapsordag); } \\
\text { Polymetallic } \mathrm{Pb}-\mathrm{Zn} \pm \mathrm{Cu}( \pm \mathrm{Ag} \text {, } \\
\mathrm{Au} \text { ) vein and stockwork } \\
\text { (Bazikskoye); } \\
\text { Carbonate-hosted fluorspar } \\
\text { (Zhurskoye) }\end{array}$ & $\begin{array}{l}\text { Russia, Southern- } \\
\text { Eastern Siberia }\end{array}$ & $\begin{array}{l}\text { Replacements related to } \\
\text { granitoids related to South } \\
\text { Siberian volcanic-plutonic } \\
\text { belt. }\end{array}$ & Devonian & $\begin{array}{l}\text { Belt interpreted as forming during rift-related } \\
\text { magmatism that formed that the South Siberian } \\
\text { volcanic-plutonic belt. }\end{array}$ \\
\hline $\begin{array}{l}\text { Agulsk } \\
\text { (AG) }\end{array}$ & $\begin{array}{l}\text { Porphyry Cu-Mo }( \pm \mathrm{Au}, \mathrm{Ag}) \\
\text { (Agulskoye, Dzhetskoye }\end{array}$ & $\begin{array}{l}\text { Russia, Southern- } \\
\text { Eastern Siberia } \\
\text { (Eastern Sayan) }\end{array}$ & $\begin{array}{l}\text { Granitoids related to } \\
\text { South Siberian volcanic- } \\
\text { plutonic belt }\end{array}$ & $\begin{array}{l}\text { Early and Middle Devonian. K-Ar } \\
\text { isotopic age for biotite granite } \\
\text { related to Irbinskoye Fe-skarn } \\
\text { deposit ranges from } 398 \text { to } 418 \\
\text { Ma. }{ }^{40} \mathrm{Ar}{ }^{39} \mathrm{Ar} \text { isotopic age of the } \\
\text { porphyry magmatism is } 400 \text { to } \\
380 \mathrm{Ma} \text {. }\end{array}$ & $\begin{array}{l}\text { Belt interpreted as forming during rift-related granitoid } \\
\text { magmatism of South Siberian volcanic-plutonic belt. }\end{array}$ \\
\hline
\end{tabular}




\begin{tabular}{|c|c|c|c|c|c|}
\hline $\begin{array}{l}\text { Name } \\
\text { (Symbol) }\end{array}$ & $\begin{array}{l}\text { Mineral Deposit Models } \\
\text { (Major Deposits) }\end{array}$ & Country, Region & $\begin{array}{l}\text { Unit or Strucutre } \\
\text { Related to Origin of } \\
\text { Metallogenic Belt }\end{array}$ & Age Range of Metallogenic Belt & $\begin{array}{l}\text { Tectonic Event for Origin of Metallogenic Belt. } \\
\text { Comments }\end{array}$ \\
\hline $\begin{array}{l}\text { Rudny Altai } \\
\text { (RA) }\end{array}$ & $\begin{array}{l}\text { Volcanogenic } \mathrm{Zn}-\mathrm{Pb}-\mathrm{Cu} \\
\text { massive sulfide (Kuroko, Altai } \\
\text { types) (Korbalihinskoye, } \\
\text { Stepnoye, Talovskoye, } \\
\text { Rubtsovskoye, Zakharovskoye, } \\
\text { Jubileinoye); } \\
\text { Barite vein (Zarechenskoye, } \\
\text { Zmeinogorskoye); } \\
\text { Volcanic-hosted metasomatite }\end{array}$ & $\begin{array}{l}\text { Russia, Southern- } \\
\text { Eastern Siberia }\end{array}$ & $\begin{array}{l}\text { Rudny Altai terrane } \\
\text { (Island arc) }\end{array}$ & Middle to Late Devonian & $\begin{array}{l}\text { Belt interpreted as forming in an island arc. Belt hosted } \\
\text { in shallow marine shelf volcanic rocks }\end{array}$ \\
\hline $\begin{array}{l}\text { Korgon- } \\
\text { Kholzun } \\
(\mathrm{KKh})\end{array}$ & $\begin{array}{l}\text { Volcanogenic-sedimentary Fe } \\
\text { (Kholzunskoye, Inskoye, } \\
\text { Beloretskoye); } \\
\text { Fe skarn, } \\
\text { Mafic-ultramafic related Ti-Fe } \\
( \pm \text { V) (Kharlovskoye); } \\
\text { Polymetallic }(\mathrm{Pb}, \mathrm{Zn}, \mathrm{Ag}) \\
\text { carbonate-hosted metasomatite } \\
\text { (Charyshskoye) }\end{array}$ & $\begin{array}{l}\text { Russia, Southern- } \\
\text { Eastern Siberia } \\
\text { (Gorny Altai area) }\end{array}$ & $\begin{array}{l}\text { Deposits related to Altai } \\
\text { volcanic-plutonic belt that } \\
\text { overlap and intrude Altai } \\
\text { and Charysh continental } \\
\text { margin turbidite terranes }\end{array}$ & Devonian to Carboniferous & $\begin{array}{l}\text { Belt interpreted as forming along an active Hercynian- } \\
\text { continental margin arc. }\end{array}$ \\
\hline $\begin{array}{l}\text { Shirgaita } \\
\text { (SH) }\end{array}$ & $\begin{array}{l}\text { Sedimentary-exhalative } \mathrm{Zn}-\mathrm{Pb} \\
\text { (SEDEX) (Shirgaita); } \\
\text { Volcanogenic } \mathrm{Zn}-\mathrm{Pb}-\mathrm{Cu} \\
\text { massive sulfide (Kuroko, Altai } \\
\text { types) (Ursulskoye) }\end{array}$ & $\begin{array}{l}\text { Russia, Southern- } \\
\text { Eastern Siberia } \\
\text { (Gorny Altai area) }\end{array}$ & $\begin{array}{l}\text { Altai volcanic-plutonic } \\
\text { belt }\end{array}$ & Early to Middle Devonian & $\begin{array}{l}\text { Belt interpreted as forming along back-arc of an island } \\
\text { arc. Belt hosted in clastic and carbonate rocks, andesite } \\
\text { and diabase porphyries, tuff, tuff breccia, } \\
\text { felsicporphyries, and siliceous tuff. }\end{array}$ \\
\hline
\end{tabular}




\begin{tabular}{|c|c|c|c|c|c|}
\hline $\begin{array}{l}\text { Name } \\
\text { (Symbol) }\end{array}$ & $\begin{array}{l}\text { Mineral Deposit Models } \\
\text { (Major Deposits) }\end{array}$ & Country, Region & $\begin{array}{l}\text { Unit or Strucutre } \\
\text { Related to Origin of } \\
\text { Metallogenic Belt }\end{array}$ & Age Range of Metallogenic Belt & $\begin{array}{l}\text { Tectonic Event for Origin of Metallogenic Belt. } \\
\text { Comments }\end{array}$ \\
\hline $\begin{array}{l}\text { Khalzan- } \\
\text { burged } \\
(\mathrm{KhZ})\end{array}$ & $\begin{array}{l}\text { Peralkaline granitoid-related } \\
\text { Nb-Zr-REE; (Ulaantolgoi); } \\
\text { Ta-Nb-REE alkaline } \\
\text { metasomatite (Shartolgoi) }\end{array}$ & Western Mongolia & $\begin{array}{l}\text { Alkaline granite plutons in } \\
\text { the Altai volcanic-plutonic } \\
\text { belt }\end{array}$ & $\begin{array}{l}\text { Interpreted as Early Devonian. K- } \\
\text { Ar amphibole isotopic age of } \\
\text { nordmarkite is } 423 \mathrm{Ma} \text {. U- } \mathrm{Pb} \text { age } \\
\text { of calcite granite is } 396 \mathrm{Ma} \text {. Sm- } \\
\text { Nd fluorite isochron age for the } \\
\text { Halzanburegtei pluton is } 325 \mathrm{Ma} \text {. }\end{array}$ & $\begin{array}{l}\text { Belt interpreted as forming along an active Andean-type } \\
\text { active continental margin. }\end{array}$ \\
\hline $\begin{array}{l}\text { Bayan-Kol } \\
(\mathrm{BK})\end{array}$ & $\begin{array}{l}\text { Magmatic nepheline (Bayan- } \\
\text { Kol, Korgere-Daba) }\end{array}$ & $\begin{array}{l}\text { Russia, Southern- } \\
\text { Eastern Siberia } \\
\text { (Tuva area); } \\
\text { Northern Mongolia }\end{array}$ & $\begin{array}{l}\text { Granitoids related to } \\
\text { South Siberian volcanic- } \\
\text { plutonic belt intruding } \\
\text { Salair terrane }\end{array}$ & $\begin{array}{l}\text { Late Mississippian and } \\
\text { Pennsylvanian. K-Ar biotite } \\
\text { average isotopic age for nepheline } \\
\text { syenite is } 310 \text { Ma. Korgere-Daba } \\
\text { pluton has age of } 296 \text { Ma; Ulan- } \\
\text { Erginsk pluton has age of } 313 \\
\text { Ma, Pichekol pluton has age of } \\
323 \mathrm{Ma} \text {. }\end{array}$ & $\begin{array}{l}\text { Belt interpreted as forming during middle Paleozoic } \\
\text { intraplate rifting. }\end{array}$ \\
\hline $\begin{array}{l}\text { Bugseingol- } \\
\text { Ovormaraat } \\
\text { (BOM) }\end{array}$ & $\begin{array}{l}\text { Magmatic nepheline } \\
\text { (Ovormaraat, Doshiin gol, } \\
\text { Beltesiin gol); } \\
\text { Peralkaline granitoid-related } \\
\text { Nb-Zr-REE (Altanboom, } \\
\text { Uranhem, Arsaan, Shignuul } \\
\text { gol, Ust gol Nb-Zr-REE; Ar } \\
\text { gol, Yarhis gol); } \\
\text { Ta-Nb-REE alkaline } \\
\text { metasomatite (Altanboom) }\end{array}$ & Northern Mongolia & $\begin{array}{l}\text { Belt is related to alkaline } \\
\text { intrusive magmatic } \\
\text { complex that occurs west } \\
\text { and south of Hovsgol } \\
\text { Lake. The alkaline } \\
\text { complex is part of an } \\
\text { alkaline magmatic aureole } \\
\text { that also occurs in eastern } \\
\text { Tuva and eastern Sayan } \\
\text { area of Russia. }\end{array}$ & $\begin{array}{l}\text { Belt interpreted as starting in } \\
\text { Early Devonian and continuing to } \\
\text { to Permian. Isotopic ages range } \\
\text { from } 400 \text { to } 396 \mathrm{Ma} \text { and } 325 \text { to } \\
300 \mathrm{Ma} \text {. }\end{array}$ & $\begin{array}{l}\text { Belt interpreted as forming along an active Andean-type } \\
\text { active continental margin. Deposits hosted in anorogenic } \\
\text { alkaline gabbro, nepheline syenite, alkaline syenite, and } \\
\text { alkaline granite. }\end{array}$ \\
\hline $\begin{array}{l}\text { Tomurtein } \\
\text { Nuruu } \\
\text { (TN) }\end{array}$ & $\begin{array}{l}\text { Clastic-sediment-hosted Sb-Au } \\
\text { (Talynmeltes) }\end{array}$ & $\begin{array}{l}\text { Southwestern } \\
\text { Mongolia }\end{array}$ & $\begin{array}{l}\text { Beitianshan-Atasbogd } \\
\text { terrane (Island arc) }\end{array}$ & $\begin{array}{l}\text { Lower to Middle Devonian. } \\
\text { Interpreted age for associated } \\
\text { greenschist facies metamorphism. }\end{array}$ & $\begin{array}{l}\text { Belt interpreted as forming during regional } \\
\text { metamorphism and vein emplacement associated with } \\
\text { accretion of Beitianshan-Atasbogd and Zhongtianshan } \\
\text { terranes. }\end{array}$ \\
\hline
\end{tabular}




\begin{tabular}{|c|c|c|c|c|c|}
\hline $\begin{array}{l}\text { Name } \\
\text { (Symbol) }\end{array}$ & $\begin{array}{l}\text { Mineral Deposit Models } \\
\text { (Major Deposits) }\end{array}$ & Country, Region & $\begin{array}{l}\text { Unit or Strucutre } \\
\text { Related to Origin of } \\
\text { Metallogenic Belt }\end{array}$ & Age Range of Metallogenic Belt & $\begin{array}{l}\text { Tectonic Event for Origin of Metallogenic Belt. } \\
\text { Comments }\end{array}$ \\
\hline $\begin{array}{l}\text { Baruunhuur } \\
\text { ai } \\
(\mathrm{BAN})\end{array}$ & $\begin{array}{l}\text { Au in shear zone and quartz } \\
\text { vein (Khaltar Uul II) }\end{array}$ & $\begin{array}{l}\text { Southwestern } \\
\text { Mongolia }\end{array}$ & $\begin{array}{l}\text { Replacements in } \\
\text { Waizunger-Baaran terrane } \\
\text { (Island arc) }\end{array}$ & Early to Middle Carboniferous & $\begin{array}{l}\text { Belt interpreted as forming during regional } \\
\text { metamorphism and vein emplacement association with } \\
\text { accretion of the Beitianshan-Atasbogd and } \\
\text { Zhongtianshan terranes. }\end{array}$ \\
\hline $\begin{array}{l}\text { Hangai } \\
\text { (HAN) }\end{array}$ & $\begin{array}{l}\text { Volcanogenic-sedimentary } \mathrm{Mn} \text {; } \\
\text { Volcanogenic-sedimentary Fe } \\
\text { (Zoogiin) }\end{array}$ & Central Mongolia & $\begin{array}{l}\text { Hangay-Dauria terrane } \\
\text { (Accretionary wedge). }\end{array}$ & Lower to Middle Devonian & $\begin{array}{l}\text { Belt interpreted as forming in marine sedimentary rocks } \\
\text { incorporated into an accretionary wedge. }\end{array}$ \\
\hline $\begin{array}{l}\text { Edrengiin } \\
\text { (ED) }\end{array}$ & $\begin{array}{l}\text { Volcanogenic } \mathrm{Cu}-\mathrm{Zn} \text { massive } \\
\text { sulfide (Urals type) (Olgii } \\
\text { nuruu); } \\
\text { Volcanogenic-sedimentary } \mathrm{Mn} \text {; } \\
\text { Volcanogenic-sedimentary Fe } \\
\text { (Olgiibulag) }\end{array}$ & $\begin{array}{l}\text { Southwestern } \\
\text { Mongolia }\end{array}$ & Edren terrane (Island arc) & Early Devonian & $\begin{array}{l}\text { Belt interpreted as forming in island arc or ophiolite } \\
\text { complex. Deposits hosted in pillow basalt and siliceous } \\
\text { rocks. }\end{array}$ \\
\hline $\begin{array}{l}\text { Bayangovi } \\
\text { (BG) }\end{array}$ & $\begin{array}{l}\text { Au in shear zone and quartz } \\
\text { vein (Bayangovi district) }\end{array}$ & Southern Mongolia & $\begin{array}{l}\text { Replacements in Govi } \\
\text { Altai terrane (Continental- } \\
\text { margin turbidite) }\end{array}$ & Devonian & $\begin{array}{l}\text { Belt interpreted as forming regional metamorphism of } \\
\text { the Govi-Altai terrane during collision with the Lake } \\
\text { terrane. }\end{array}$ \\
\hline $\begin{array}{l}\text { Bayanleg } \\
\text { (BL) }\end{array}$ & $\begin{array}{l}\text { Besshi } \mathrm{Cu}-\mathrm{Zn}-\mathrm{Ag} \text { massive } \\
\text { sulfide (Bayantsagaan 1) }\end{array}$ & Southern Mongolia & $\begin{array}{l}\text { Bayanleg terrane } \\
\text { (Accretionary wedge - } \\
\text { type B) }\end{array}$ & Early Devonian & $\begin{array}{l}\text { Belt interpreted as forming in marine sedimentary rocks } \\
\text { incorporated into an accretionary wedge. }\end{array}$ \\
\hline $\begin{array}{l}\text { Ulziit } \\
\text { (UZ) }\end{array}$ & $\begin{array}{l}\text { Au in shear zone and quartz } \\
\text { vein (Olon Ovoot) }\end{array}$ & Southern Mongolia & $\begin{array}{l}\text { Replacements in Govi } \\
\text { Altai terrane (Continental- } \\
\text { margin turbidite) }\end{array}$ & Devonian(?) & $\begin{array}{l}\text { Belt interpreted as forming regional metamorphism of } \\
\text { Govi-Altai terrane during collision with the Idermeg } \\
\text { terrane. }\end{array}$ \\
\hline $\begin{array}{l}\text { Sulinheer } \\
\text { (Sul) }\end{array}$ & Podiform chromite (Sulinheer) & $\begin{array}{l}\text { Southeastern } \\
\text { Mongolia }\end{array}$ & $\begin{array}{l}\text { Solon terrane } \\
\text { (Accretionary wedge) }\end{array}$ & Carboniferous(?) & $\begin{array}{l}\text { Belt interpreted as forming in a middle Paleozoic } \\
\text { ophlolite complex that was structurally incorporated into } \\
\text { an accretionary wedge. }\end{array}$ \\
\hline $\begin{array}{l}\text { Hegenshan } \\
\text { (Heg) }\end{array}$ & $\begin{array}{l}\text { Podiform chromite } \\
\text { (Hegenshan-3756) }\end{array}$ & $\begin{array}{l}\text { Northeastern } \\
\text { China }\end{array}$ & $\begin{array}{l}\text { Dunite dikes in } \\
\text { Heilongjiang terrane } \\
\text { (Accretionary wedge) (too } \\
\text { small to show at } 10 \mathrm{M} \\
\text { scale) }\end{array}$ & $\begin{array}{l}\text { Middle Devonian. K-Ar isotopic } \\
\text { age of peridotite is } 380 \mathrm{Ma} \text {. }\end{array}$ & $\begin{array}{l}\text { Belt interpreted as forming in a middle Paleozoic } \\
\text { ophlolite complex that was structurally incorporated into } \\
\text { an accretionary wedge. }\end{array}$ \\
\hline $\begin{array}{l}\text { Yaroslavka } \\
\text { (YA) }\end{array}$ & $\begin{array}{l}\text { Fluorite greisen (Voznesenka- } \\
\text { II); } \\
\text { Sn-W greisen, stockwork, and } \\
\text { quartz vein (Yaroslavskoe) }\end{array}$ & Russia, Far East & $\begin{array}{l}\text { Granitoids intruding } \\
\text { Voznesenka terrane } \\
\text { (Passive continental } \\
\text { margin) }\end{array}$ & $\begin{array}{l}\text { Late Cambrian and though } \\
\text { Devonian. Granitoids have K-Ar } \\
\text { isotopic ages of } 440 \text { to } 396 \mathrm{Ma} \text {. }\end{array}$ & $\begin{array}{l}\text { Belt interpreted as forming in a collisional arc that } \\
\text { formed in a fragment of Gondwanaland. Host } \\
\text { leucogranite plutons interpreted as forming during early } \\
\text { Paleozoic collision of the Voznesenka and Kabarga } \\
\text { terranes. Deposit-related granitoids intrude Cambrian } \\
\text { clastic rocks and limestone. }\end{array}$ \\
\hline
\end{tabular}




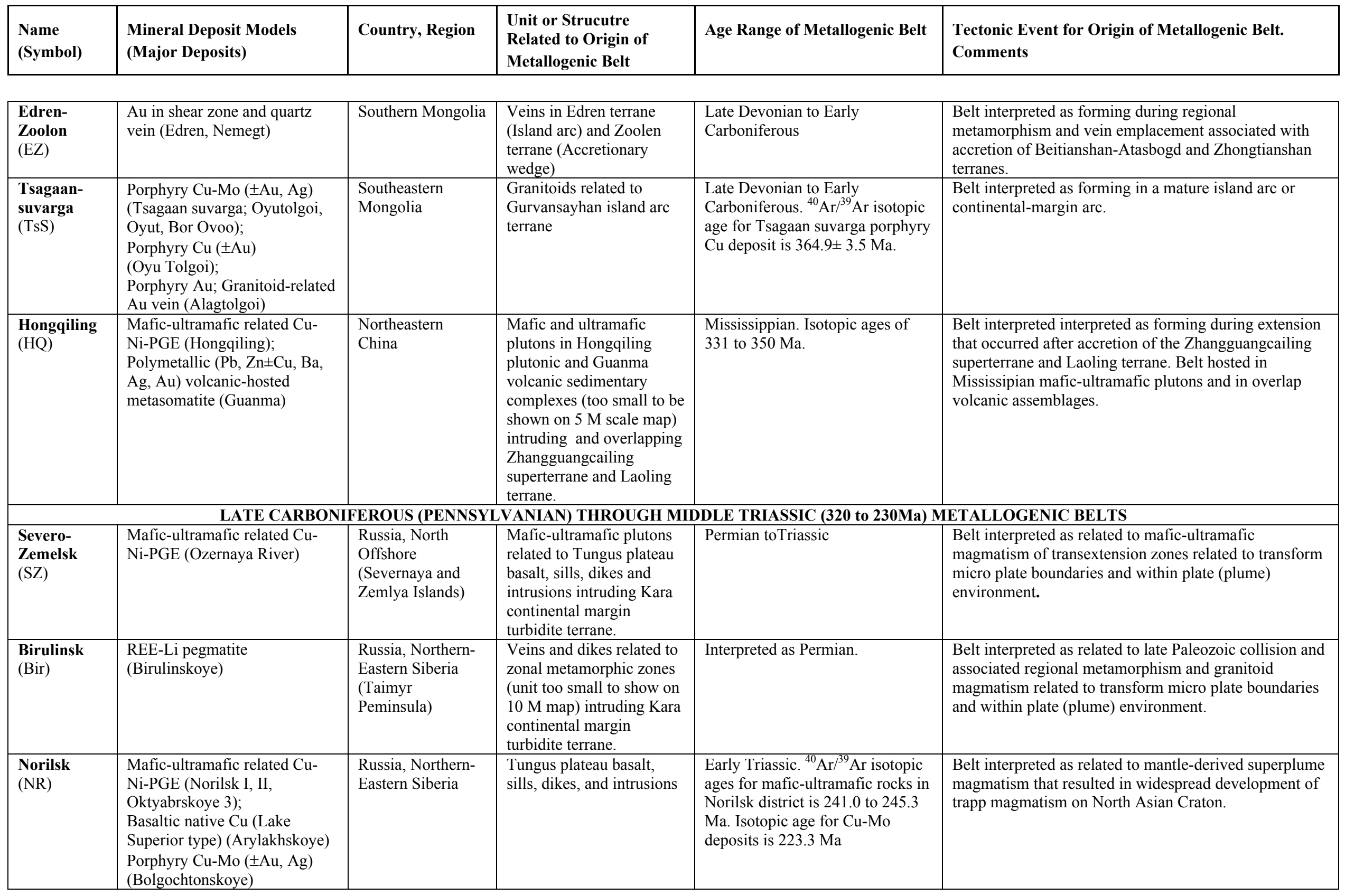




\begin{tabular}{|c|c|c|c|c|c|}
\hline $\begin{array}{l}\text { Name } \\
\text { (Symbol) }\end{array}$ & $\begin{array}{l}\text { Mineral Deposit Models } \\
\text { (Major Deposits) }\end{array}$ & Country, Region & $\begin{array}{l}\text { Unit or Strucutre } \\
\text { Related to Origin of } \\
\text { Metallogenic Belt }\end{array}$ & Age Range of Metallogenic Belt & $\begin{array}{l}\text { Tectonic Event for Origin of Metallogenic Belt. } \\
\text { Comments }\end{array}$ \\
\hline $\begin{array}{l}\text { Kureisko- } \\
\text { Tungsk } \\
(\mathrm{KT})\end{array}$ & $\begin{array}{l}\text { Fe skarn (Suringdakonskoye); } \\
\text { Mafic-ultramafic related Cu- } \\
\text { Ni-PGE (Bilchany River); } \\
\text { Metamorphic graphite } \\
\text { (Noginskoye) }\end{array}$ & $\begin{array}{l}\text { Russia, Northern- } \\
\text { Eastern Siberia }\end{array}$ & $\begin{array}{l}\text { Replacements and plutons } \\
\text { related to Tungus plateau } \\
\text { basalt, sills, dikes, and } \\
\text { intrusions }\end{array}$ & Permian to Triassic & $\begin{array}{l}\text { Belt interpreted as related to mantle superplume } \\
\text { magmatism that resulted in widespread development of } \\
\text { trapp magmatism on North Asian Craton along the long- } \\
\text { lived West-Siberian rift system and Yenisei } \\
\text { sublongitudinal major fault. }\end{array}$ \\
\hline $\begin{array}{l}\text { West } \\
\text { Verkho- } \\
\text { yansk } \\
\text { (WV) }\end{array}$ & $\begin{array}{l}\text { Au in black shale } \\
\text { (Mangazeika); } \\
\text { Polymetallic } \mathrm{Pb}-\mathrm{Zn} \pm \mathrm{Cu}( \pm \mathrm{Ag} \text {, } \\
\text { Au) vein and stockwork } \\
\text { (Kysyltas) }\end{array}$ & $\begin{array}{l}\text { Russia, East- } \\
\text { Central Yakutia } \\
\text { (Verkhoyansk } \\
\text { area) }\end{array}$ & $\begin{array}{l}\text { North Asian Craton } \\
\text { Margin - Verkhoyansk } \\
\text { fold and thrust belt } \\
\text { (Passive continental } \\
\text { margin). }\end{array}$ & $\begin{array}{l}\text { Interpreted as Late Carboniferous } \\
\text { to Early Permian. Lead from the } \\
\text { ores of the Mangazeika deposit } \\
\text { have isotopic age of } 183 \text { to } 120 \\
\text { Ma. Oldest ages of } 183 \text { to } 174 \mathrm{Ma} \\
\text { obtained from the upper section } \\
\text { of Carboniferous host rock. }\end{array}$ & $\begin{array}{l}\text { Belt interpreted as forming along passive continental } \\
\text { margin of North Asian Craton during rifting. }\end{array}$ \\
\hline $\begin{array}{l}\text { Central } \\
\text { Tungusk } \\
(\mathrm{CT})\end{array}$ & $\begin{array}{l}\text { Hydrothermal Iceland spar } \\
\text { (Krutoye, Gonchak) }\end{array}$ & Russia, Yakutia & $\begin{array}{l}\text { Replacements related to } \\
\text { Tungus plateau basalt, } \\
\text { sills, dikes, and intrusions }\end{array}$ & $\begin{array}{l}\text { Early Triassic. }{ }^{40} \mathrm{Ar} /{ }^{39} \mathrm{Ar} \text { isotopic } \\
\text { age for basalt from the northern } \\
\text { part of the Tungussk syncline is } \\
248.0 \text { to } 248.9 \mathrm{Ma} \text {. }\end{array}$ & $\begin{array}{l}\text { Belt interpreted as related to widespread development of } \\
\text { trapp magmatism on North Asian Craton. Belt coincides } \\
\text { with Triassic tholeiite volcanic and intrusive rocks. }\end{array}$ \\
\hline $\begin{array}{l}\text { Angara-Ilim } \\
\text { (AI) }\end{array}$ & $\begin{array}{l}\text { Fe skarn (Korshunovskoye); } \\
\text { REE ( } \pm \mathrm{Ta}, \mathrm{Nb}, \mathrm{Fe}) \text { carbonatite } \\
\text { (Chuktukonskoye); } \\
\text { Weathering crust carbonatite } \\
\text { REE-Zr-Nb-Li } \\
\text { (Chuktukonskoye) }\end{array}$ & $\begin{array}{l}\text { Russia, Southwest } \\
\text { Yakutia }\end{array}$ & $\begin{array}{l}\text { Replacements related to } \\
\text { Tungus plateau basalt, } \\
\text { sills, dikes, and intrusions } \\
\text { intruding North Asian } \\
\text { Craton }\end{array}$ & $\begin{array}{l}\text { Late Permian to Early Triassic(?). } \\
\text { Isotopic age of related igneous } \\
\text { rock ranges from } 260 \text { to } 200 \mathrm{Ma} \text {. }\end{array}$ & $\begin{array}{l}\text { Belt interpreted as related to widespread development of } \\
\text { trapp magmatism on North Asian Craton. Fe skarn } \\
\text { deposits associated with Triassic explosive and intrusive } \\
\text { basaltic trapp complexes in diatremes. REE-Ta-Nb } \\
\text { carbonatite deposits associated with alkali-ultramafic } \\
\text { intrusions. }\end{array}$ \\
\hline $\begin{array}{l}\text { Barlaksk } \\
(\mathrm{BA})\end{array}$ & $\begin{array}{l}\text { W-Mo-Be greisen, stockwork, } \\
\text { and quartz vein } \\
\text { (Kolyvanskoye) }\end{array}$ & $\begin{array}{l}\text { Eastern Siberia, } \\
\text { Russia }\end{array}$ & $\begin{array}{l}\text { Replacements related to } \\
\text { granitoids of Belokurikha } \\
\text { plutonic belt (too small to } \\
\text { show at } 10 \mathrm{M} \text { scale) } \\
\text { intruding Kolyvan-Tom } \\
\text { back-arc basin }\end{array}$ & $\begin{array}{l}\text { Middle Triassic. Isotopic ages of } \\
\text { deposit-related leucogranitic } \\
\text { intrusions are } 235.9 \text { to } 232.0 \mathrm{Ma} \text {. }\end{array}$ & $\begin{array}{l}\text { Belt interpreted as related to interplate rifting and } \\
\text { associated strike-slip faulting. Belt hosted in intraplate } \\
\text { granitoids in the Barlak pluton that intruded along } \\
\text { strike-slip faults. }\end{array}$ \\
\hline
\end{tabular}




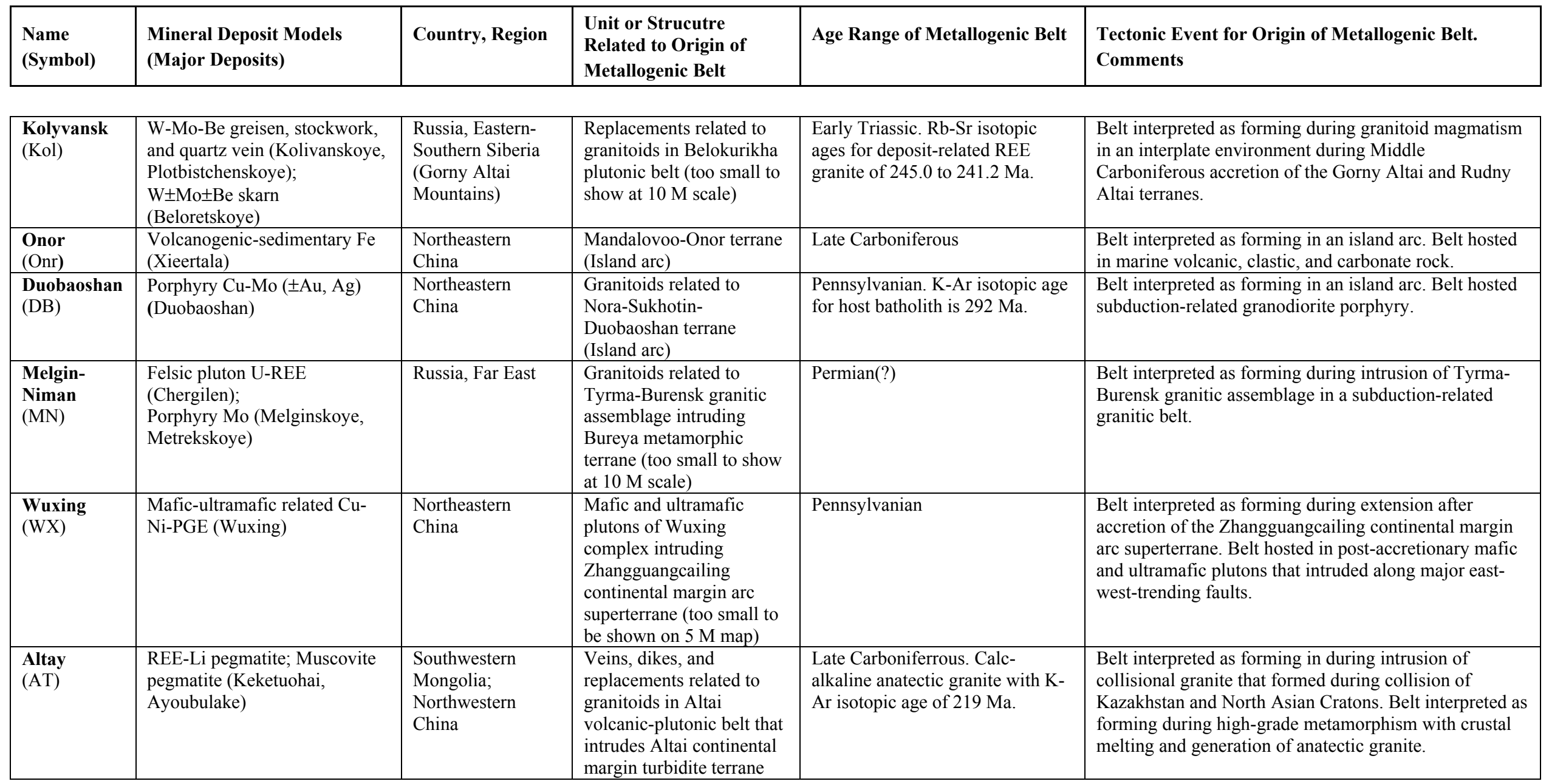




\begin{tabular}{|c|c|c|c|c|c|}
\hline $\begin{array}{l}\text { Name } \\
\text { (Symbol) }\end{array}$ & $\begin{array}{l}\text { Mineral Deposit Models } \\
\text { (Major Deposits) }\end{array}$ & Country, Region & $\begin{array}{l}\text { Unit or Strucutre } \\
\text { Related to Origin of } \\
\text { Metallogenic Belt }\end{array}$ & Age Range of Metallogenic Belt & $\begin{array}{l}\text { Tectonic Event for Origin of Metallogenic Belt. } \\
\text { Comments }\end{array}$ \\
\hline $\begin{array}{l}\text { Battsengel- } \\
\text { Uyanga- } \\
\text { Erdenedalai } \\
\text { (BUE) }\end{array}$ & $\begin{array}{l}\text { Granitoid-related Au vein } \\
\text { (Mongot, Battsengel, Uyanga } \\
\text { groups, Sharga Ovoo, Tsagaan } \\
\text { Ovoo) }\end{array}$ & Central Mongolia & $\begin{array}{l}\text { Small stitching plutons } \\
\text { that formed in early stage } \\
\text { of intrusion of Hangay } \\
\text { plutonic belt that intrudes } \\
\text { Hangay-Dauria and Onon } \\
\text { accretionary wedge } \\
\text { terranes. }\end{array}$ & Late Carboniferous to Permian & $\begin{array}{l}\text { Belt interpreted as forming in subduction-related gabbro, } \\
\text { diorite, and granodiorite stocks and dikes along along } \\
\text { the North Govi active continental margin arc. }\end{array}$ \\
\hline $\begin{array}{l}\text { Orhon- } \\
\text { Selenge } \\
(\mathrm{OS})\end{array}$ & $\begin{array}{l}\text { Porphyry Cu-Mo ( } \pm \mathrm{Au}, \mathrm{Ag}) \\
\text { (Erdenetiin Ovoo, Central, } \\
\text { Oyut; Shand; Zuiliin gol) }\end{array}$ & Central Mongolia & $\begin{array}{l}\text { Granitoids in Selenga } \\
\text { sedimentary-volcanic } \\
\text { plutonic belt. }\end{array}$ & $\begin{array}{l}\text { Triassic. Quartz-sericite } \\
\text { metasomatite of the Erdenetiin } \\
\text { Ovoo deposit has K-Ar isotopic } \\
\text { age } 210 \text { to } 190 \mathrm{Ma} \text {. Explosive } \\
\text { breccia has age if } 210 \mathrm{Ma} \text {. K-Ar } \\
\text { age of deposit-related granite } \\
\text { ranges from } 185 \mathrm{Ma} \text { to } 240 \text { to } 250 \\
\mathrm{Ma}{ }^{40} \mathrm{Ar} /{ }^{39} \mathrm{Ar} \text { isochron isotopic } \\
\text { age of } 207 \pm 2 \mathrm{Ma} \text { for white mica } \\
\text { from highest grade part of } \\
\text { Erdenet mine. }\end{array}$ & $\begin{array}{l}\text { Belt interpreted as forming during oblique subduction of } \\
\text { oceanic crust of the Mongol-Okhotsk paleoocean under } \\
\text { the southern margin of the Siberian continent. Basaltic } \\
\mathrm{Cu} \text { hosted in basalt and trachybasalt of the mafic } \\
\text { volcanic rock in the Permian Khanui Seri Formation. }\end{array}$ \\
\hline
\end{tabular}




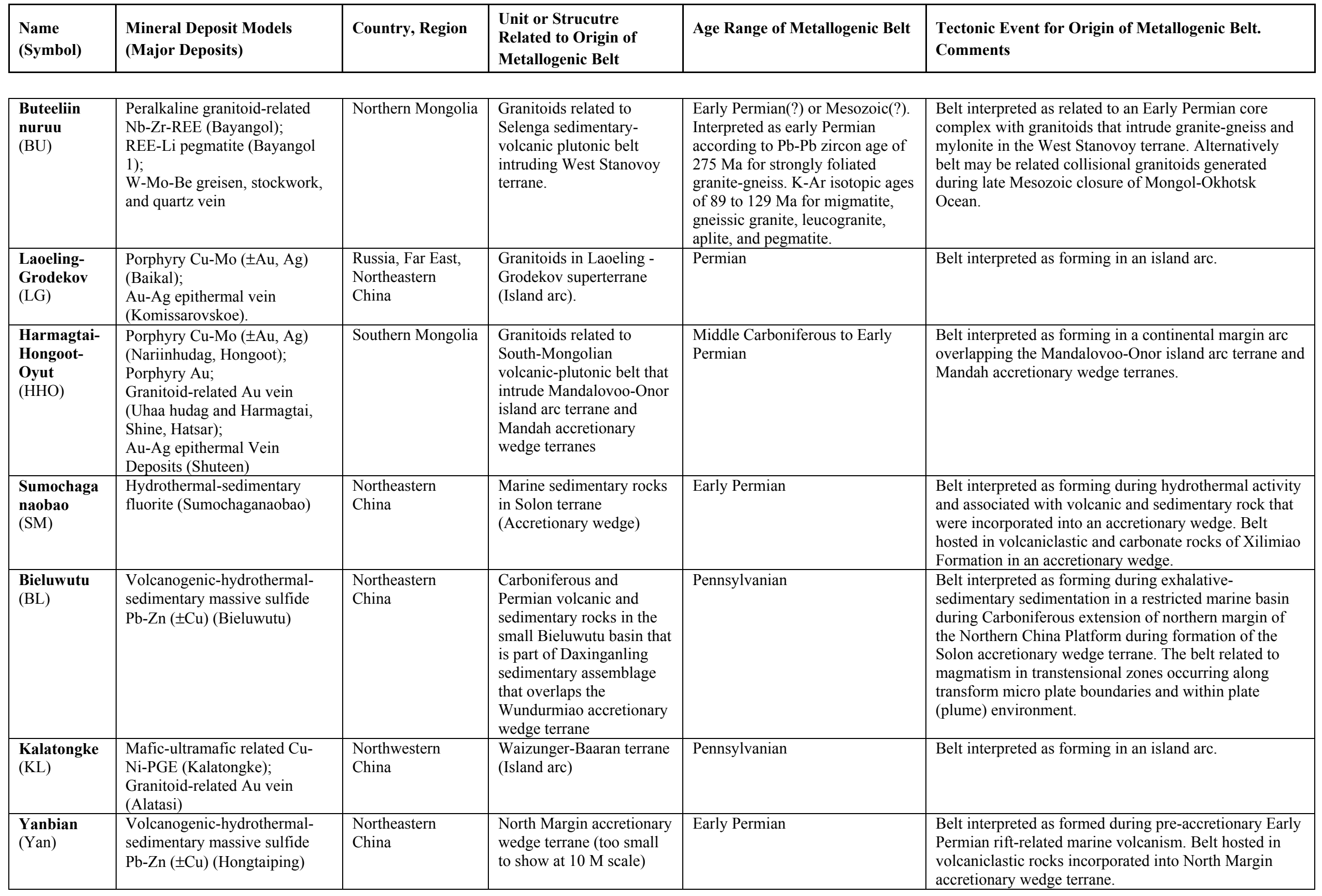




\begin{tabular}{|c|c|c|c|c|c|}
\hline $\begin{array}{l}\text { Name } \\
\text { (Symbol) }\end{array}$ & $\begin{array}{l}\text { Mineral Deposit Models } \\
\text { (Major Deposits) }\end{array}$ & Country, Region & $\begin{array}{l}\text { Unit or Strucutre } \\
\text { Related to Origin of } \\
\text { Metallogenic Belt }\end{array}$ & Age Range of Metallogenic Belt & $\begin{array}{l}\text { Tectonic Event for Origin of Metallogenic Belt. } \\
\text { Comments }\end{array}$ \\
\hline $\begin{array}{l}\text { Zibe } \\
\text { (ZB) }\end{array}$ & Sedimentary bauxite (Zibe) & Northern China & $\begin{array}{l}\text { Sino-Korea platform } \\
\text { sedimentary cover } \\
\text { (Proterozoic through } \\
\text { Triassic) overlapping } \\
\text { Sino-Korean Craton - } \\
\text { West Liaoning-Hebei- } \\
\text { Shanxi terrane. }\end{array}$ & Late Permian & $\begin{array}{l}\text { Belt formed during weathering of metamorphic rocks of } \\
\text { the Northern China Platform. Bauxite deposits hosted in } \\
\text { karst and lagoonal basins in a littoral shallow sea. }\end{array}$ \\
\hline $\begin{array}{l}\text { Mino- } \\
\text { Tamba- } \\
\text { Chugoku } \\
\text { (MTC) }\end{array}$ & $\begin{array}{l}\text { Volcanogenic-sedimentary Mn } \\
\text { (Hamayokokawa); } \\
\text { Podiform chromite } \\
\text { (Wakamatsu); } \\
\text { Besshi Cu-Zn-Ag massive } \\
\text { sulfide (Yanahara) }\end{array}$ & Japan & $\begin{array}{l}\text { Mino Tamba Chichibu } \\
\text { terrane (Accretionary } \\
\text { wedge) }\end{array}$ & $\begin{array}{l}\text { Interpreted as Permian (or older) } \\
\text { to Jurassic. }\end{array}$ & $\begin{array}{l}\text { Belt is hosted in an accretionary wedge complex } \\
\text { composed of marine sedimentary and volcanic rock, and } \\
\text { fragments of oceanic crust with ultramafic rock. Besshi } \\
\text { deposits are interpreted as forming along a spreading } \\
\text { ridge. The belt contains fragments of oceanic crust with } \\
\text { podiform chromite deposits hosted in ultramafic rocks, } \\
\text { and chert-hosted Mn deposits. Deposits and host rocks } \\
\text { were subsequently incorporated into an accretionary } \\
\text { wedge. }\end{array}$ \\
\hline $\begin{array}{l}\text { Hitachi } \\
\text { (Hit) }\end{array}$ & $\begin{array}{l}\text { Volcanogenic } \mathrm{Zn}-\mathrm{Pb}-\mathrm{Cu} \\
\text { massive sulfide (Kuroko, Altai } \\
\text { types) (Hitachi) }\end{array}$ & Japan & $\begin{array}{l}\text { South Kitakami terrane } \\
\text { (Island arc) }\end{array}$ & Permian & Belt interpreted as forming in an island arc. \\
\hline \multicolumn{6}{|c|}{ LATE TRIASSIC THROUGH EARLY JURASSIC (230 to 175 Ma) METALLOGENIC BELTS } \\
\hline $\begin{array}{l}\text { North } \\
\text { Taimyr } \\
\text { (NT) }\end{array}$ & $\begin{array}{l}\text { W-Mo-Be greisen, stockwork, } \\
\text { and quartz vein (Kolomeitseva } \\
\text { River); } \\
\text { W } \pm \text { Mo } \pm \text { Be skarn } \\
\text { Morzhovoye); } \\
\text { Porphyry Cu-Mo ( } \pm \mathrm{Au}, \mathrm{Ag}) \\
\text { (Mamont River) }\end{array}$ & $\begin{array}{l}\text { Russia, Northern- } \\
\text { Eastern Siberia } \\
\text { (Taimyr } \\
\text { Peminsula) }\end{array}$ & $\begin{array}{l}\text { Replacements associated } \\
\text { with granitoids (too small } \\
\text { to show at } 5 \mathrm{M} \text { scale) } \\
\text { intruding Permian-Triassic } \\
\text { volcanic and sedimentary } \\
\text { rocks of Lenivaya- } \\
\text { Chelyuskin sedimentary } \\
\text { assemblage, Central } \\
\text { Taimyr superterrane, Kara } \\
\text { terrane. }\end{array}$ & $\begin{array}{l}\text { Middle and Late Triassic. Age of } \\
\text { deposit-related granitoids is about } \\
223 \text { to } 233 \text { Ma. }\end{array}$ & $\begin{array}{l}\text { Belt interpreted as forming during generation of } \\
\text { granitoids during and after collision between the } \\
\text { Siberian and Kara continents. Belt hosted in intrusions } \\
\text { in tectonic blocks bounded by post-orogenic faults. }\end{array}$ \\
\hline
\end{tabular}




\begin{tabular}{|c|c|c|c|c|c|}
\hline $\begin{array}{l}\text { Name } \\
\text { (Symbol) }\end{array}$ & $\begin{array}{l}\text { Mineral Deposit Models } \\
\text { (Major Deposits) }\end{array}$ & Country, Region & $\begin{array}{l}\text { Unit or Strucutre } \\
\text { Related to Origin of } \\
\text { Metallogenic Belt }\end{array}$ & Age Range of Metallogenic Belt & $\begin{array}{l}\text { Tectonic Event for Origin of Metallogenic Belt. } \\
\text { Comments }\end{array}$ \\
\hline $\begin{array}{l}\text { Mongol } \\
\text { Altai } \\
\text { (MA) }\end{array}$ & $\begin{array}{l}\text { W-Mo-Be greisen, stockwork, } \\
\text { and quartz vein (Ulaan Uul, } \\
\text { Tsunheg) }\end{array}$ & Western Mongolia & $\begin{array}{l}\text { Small bodies of } \\
\text { leucogranite (too small to } \\
\text { show at } 5 \mathrm{M} \text { scale) that } \\
\text { intrude Altai and Hovd } \\
\text { Hovd terranes }\end{array}$ & $\begin{array}{l}\text { Interpreted as Late Triassic to } \\
\text { Early Jurassic. }\end{array}$ & $\begin{array}{l}\text { Belt interpreted as forming during Mesozoic intraplate } \\
\text { rifting related to magmatism along transextension zones } \\
\text { along transform micro plate boundaries and within plate } \\
\text { (plume) environment. }\end{array}$ \\
\hline $\begin{array}{l}\text { Chergak } \\
\text { (ChG) }\end{array}$ & $\begin{array}{l}\text { Ni-Co arsenide vein (Chergak, } \\
\text { Tolailyk) }\end{array}$ & $\begin{array}{l}\text { Russia, Southern- } \\
\text { Eastern Siberia } \\
\text { (Tuva region) }\end{array}$ & $\begin{array}{l}\text { Veins and associated } \\
\text { gabbro intrusions related } \\
\text { to Akchem, Severo- } \\
\text { Tannuola, and Eldigkhem } \\
\text { faults cutting Khemchik- } \\
\text { Sistigkhem basin, Tuva } \\
\text { molasse basin, and West } \\
\text { Sayan terrane }\end{array}$ & Interpreted as Triassic. & $\begin{array}{l}\text { Belt interpreted as forming during Mesozoic intraplate } \\
\text { rifting that resulted in magmatism along transextension } \\
\text { zones along transform micro plate boundaries and within } \\
\text { plate (plume) environment. }\end{array}$ \\
\hline $\begin{array}{l}\text { Khovuak- } \\
\text { sinsk } \\
(\mathrm{KhA})\end{array}$ & $\begin{array}{l}\text { Ni-Co arsenide vein (Hovu- } \\
\text { Aksinskoye, Uzun-Oy) }\end{array}$ & $\begin{array}{l}\text { Russia, Southern- } \\
\text { Eastern Siberia } \\
\text { (Tuva region) }\end{array}$ & $\begin{array}{l}\text { Veins related to Ubsunur- } \\
\text { Bayankol fault cutting } \\
\text { Tuva molasse basin and } \\
\text { Tannuola subterrane }\end{array}$ & Interpreted as Triassic. & $\begin{array}{l}\text { Belt interpreted as forming during Mesozoic intraplate } \\
\text { rifting that resulted in magmatism along transextension } \\
\text { zones along transform micro plate boundaries and within } \\
\text { plate (plume) environment. }\end{array}$ \\
\hline $\begin{array}{l}\text { Ulug- } \\
\text { Tanzek } \\
\text { (UT) }\end{array}$ & $\begin{array}{l}\text { Ta-Nb-REE alkaline } \\
\text { metasomatite (Ulug-Tanzek) }\end{array}$ & $\begin{array}{l}\text { Russia, Southern- } \\
\text { Eastern Siberia } \\
\text { (Tuva region) }\end{array}$ & $\begin{array}{l}\text { Replacements related to } \\
\text { Ulug-Tanzek granite } \\
\text { intrusion (to small to show } \\
\text { on } 10 \mathrm{M} \text { map) that } \\
\text { intrudes Sangilen terrane } \\
\text { (passive continental } \\
\text { margin) }\end{array}$ & $\begin{array}{l}\text { Late Triassic. Isotopic ages of } 231 \\
\text { to } 228 \text { Ma for Bren massive, } 217 \\
\text { Ma for Ulug-Tanzek complex, } \\
\text { and } 209 \text { Ma for younger related } \\
\text { intrusive rocks }\end{array}$ & $\begin{array}{l}\text { Belt interpreted as forming during intraplate tectonism } \\
\text { and magmatism in an intraplate rift setting. Belt hosted } \\
\text { in alkali granite plutons in the Ulug-Tanzek intrusive } \\
\text { complex. The belt is characterized by magmatic rocks } \\
\text { related to transform micro plate boundaries and within } \\
\text { plate (plume) environment. }\end{array}$ \\
\hline \multicolumn{3}{|c|}{$\begin{array}{l}\text { Orhon-Selenge } \\
\text { (OS) }\end{array}$} & \multicolumn{3}{|c|}{ Started in Late Carboniferrous and continued into Middle Triassic } \\
\hline
\end{tabular}




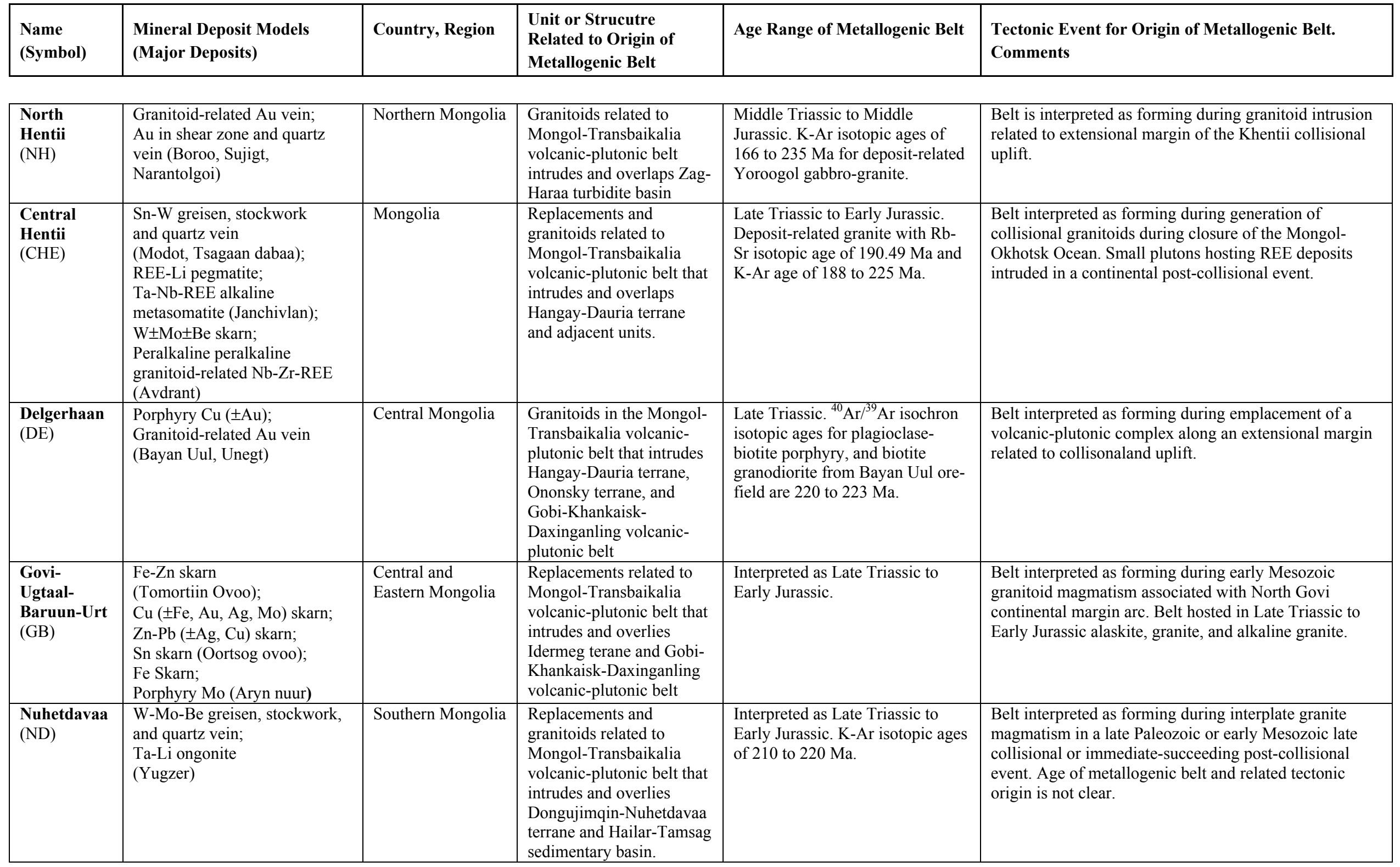




\begin{tabular}{|c|c|c|c|c|c|}
\hline $\begin{array}{l}\text { Name } \\
\text { (Symbol) }\end{array}$ & $\begin{array}{l}\text { Mineral Deposit Models } \\
\text { (Major Deposits) }\end{array}$ & Country, Region & $\begin{array}{l}\text { Unit or Strucutre } \\
\text { Related to Origin of } \\
\text { Metallogenic Belt }\end{array}$ & Age Range of Metallogenic Belt & $\begin{array}{l}\text { Tectonic Event for Origin of Metallogenic Belt. } \\
\text { Comments }\end{array}$ \\
\hline $\begin{array}{l}\text { Wulashan- } \\
\text { Zhangbei } \\
\text { (WZ) }\end{array}$ & $\begin{array}{l}\text { Alkaline complex-hosted } \mathrm{Au} ; \\
\text { (Dongping); } \\
\text { Au potassium metasomatite } \\
\text { (Hadamen); } \\
\text { Granitoid-related Au vein }\end{array}$ & Northern China & $\begin{array}{l}\text { Granitoids related to } \\
\text { Alashan-Yinshan Triassic } \\
\text { plutonic belt (too small to } \\
\text { show at } 10 \mathrm{M} \text { scale) } \\
\text { intruding Sino-Korean } \\
\text { Craton - Erduosi terrane, } \\
\text { Solon terrane, and } \\
\text { adjacent units }\end{array}$ & $\begin{array}{l}\text { Middle Jurassic. }{ }^{40} \mathrm{Ar}^{39} \mathrm{Ar} \\
\text { isotopic ages is } 327 \mathrm{Ma} \text { and } 157 \\
\text { to } 177 \mathrm{Ma} \text { for intrusion and } \\
\text { deposit potassic feldspar, } \\
\text { respectively. }\end{array}$ & $\begin{array}{l}\text { Belt interpreted as forming during granitoids generated } \\
\text { above a mantle plume in an extensional tectonic setting. } \\
\text { Belt related to Late Triassic to Early Jurassic alkaline to } \\
\text { subalkaline granite. }\end{array}$ \\
\hline $\begin{array}{l}\text { Fanshan } \\
\text { (FS) }\end{array}$ & $\begin{array}{l}\text { Magmatic and metasomatic } \\
\text { apatite (Fanshan) }\end{array}$ & Northern China & $\begin{array}{l}\text { Mafic-ultramafic plutons } \\
\text { occurring along major } \\
\text { fault cutting Sino-Korea } \\
\text { platform sedimentary } \\
\text { cover and Sino-Korean } \\
\text { Craton - West Liaoning- } \\
\text { Hebei-Shanxi terrane } \\
\end{array}$ & $\begin{array}{l}\text { Late Triassic. Rb-Sr isochron age } \\
\text { of } 218.8 \mathrm{Ma} \text {. }\end{array}$ & $\begin{array}{l}\text { Belt interpreted as forming during intrusion of intraplate } \\
\text { mafic-ultramafic plutons associated with the subduction } \\
\text { of Kula plate under the Eurasian plate. }\end{array}$ \\
\hline $\begin{array}{l}\text { Gyeonggi } \\
\text { (GA) }\end{array}$ & $\begin{array}{l}\text { Mafic-ultramafic related Ti-Fe } \\
( \pm \mathrm{V}) \text { (Soyounpyong-do); } \\
\text { Polymetallic } \mathrm{Pb}-\mathrm{Zn} \pm \mathrm{Cu}( \pm \mathrm{Ag}, \\
\text { Au) vein and stockwork } \\
(\mathrm{Chilbo}) \\
\mathrm{W} \pm \mathrm{Mo} \pm \mathrm{Be} \text { skarn (Bupyung) }\end{array}$ & South Korea & $\begin{array}{l}\text { Mafic-ultramafic plutons } \\
\text { and granitoids related to } \\
\text { Daebu Granite belt } \\
\text { intruding South China } \\
\text { Craton - Gyenggi terrane }\end{array}$ & Interpreted as Early Jurassic. & $\begin{array}{l}\text { Belt is related to magmatism along transextension zones } \\
\text { along transform micro plate boundaries and within plate } \\
\text { (plume) environment. Ti-Fe deposits interpreted as } \\
\text { forming during intrusion of mafic and ultramafic plutons } \\
\text { associated with Late Jurassic to Early Cretaceous Daebo } \\
\text { orogeny. Polymetallic vein deposits formed during } \\
\text { hydrothermal fluid activity, and skarns formed during } \\
\text { contact metasomatism along contact zones of } \\
\text { hornblende biotite granite and dikes. }\end{array}$ \\
\hline \multicolumn{3}{|c|}{ Mino-Tamba-Chugoku (MTC) } & \multicolumn{3}{|c|}{ Started in Late Carboniferous through Middle Triassic } \\
\hline
\end{tabular}




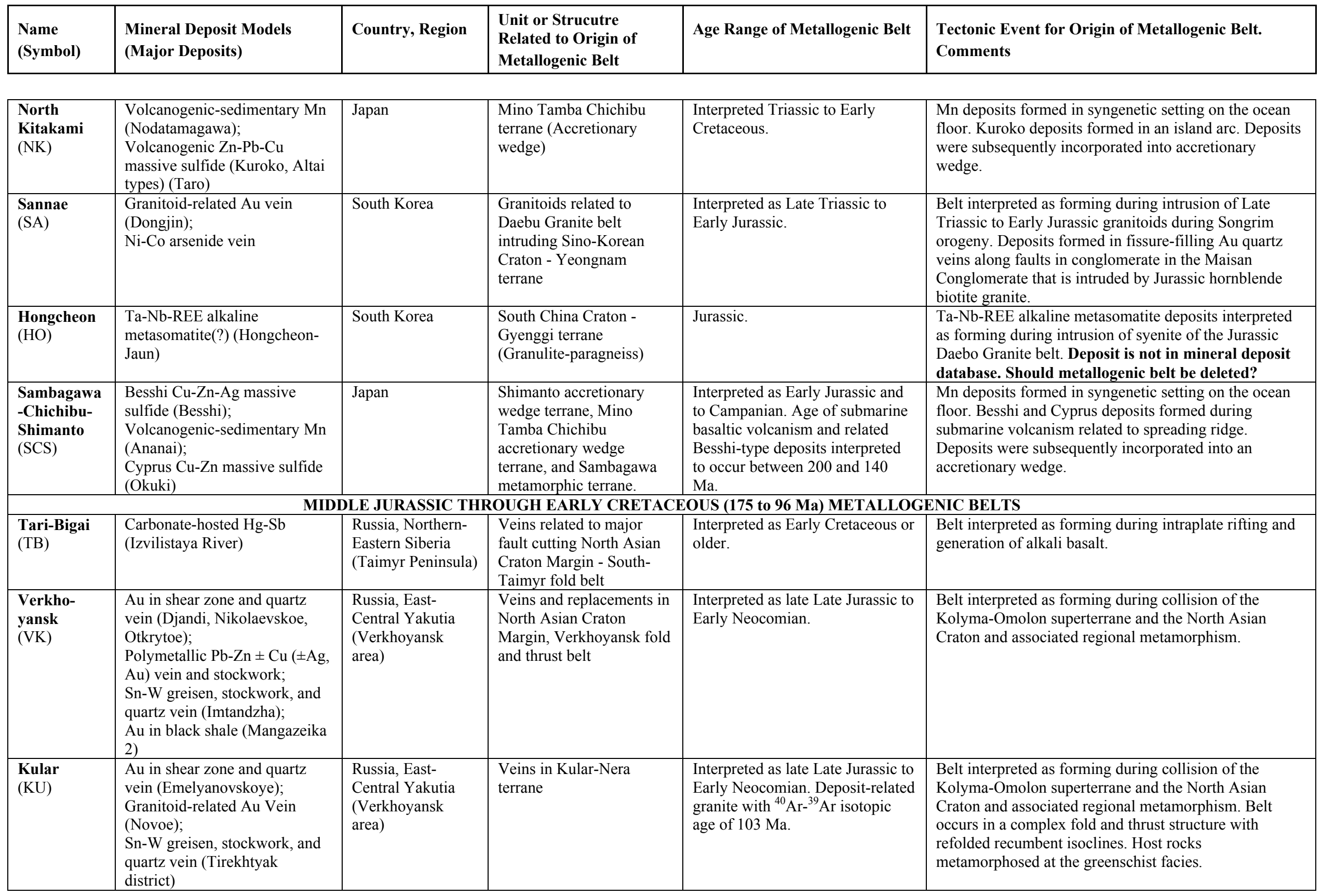




\begin{tabular}{|c|c|c|c|c|c|}
\hline $\begin{array}{l}\text { Name } \\
\text { (Symbol) }\end{array}$ & $\begin{array}{l}\text { Mineral Deposit Models } \\
\text { (Major Deposits) }\end{array}$ & Country, Region & $\begin{array}{l}\text { Unit or Strucutre } \\
\text { Related to Origin of } \\
\text { Metallogenic Belt }\end{array}$ & Age Range of Metallogenic Belt & $\begin{array}{l}\text { Tectonic Event for Origin of Metallogenic Belt. } \\
\text { Comments }\end{array}$ \\
\hline $\begin{array}{l}\text { Adycha- } \\
\text { Nera } \\
(\mathrm{AN})\end{array}$ & $\begin{array}{l}\text { Au in shear zone and quartz } \\
\text { vein (Uchui); } \\
\text { Sn-W greisen, stockwork, and } \\
\text { quartz vein (Imtachan); } \\
\text { Granitoid-related Au vein } \\
\text { (Delyuvialnoe) }\end{array}$ & $\begin{array}{l}\text { Russia, East- } \\
\text { Central Yakutia } \\
\text { (Verkhoyansk } \\
\text { area) }\end{array}$ & $\begin{array}{l}\text { Veins in Kular-Nera } \\
\text { terrane }\end{array}$ & $\begin{array}{l}\text { Interpreted as late Late Jurassic to } \\
\text { Early Neocomian. }\end{array}$ & $\begin{array}{l}\text { Belt interpreted as forming in two stages: (1) initial } \\
\text { accumulation of disseminated Au in the late Paleozoic } \\
\text { early Lower Mesozoic black slate; and (2) mobilization } \\
\text { during regional metamorphism and collisional granitoid } \\
\text { intrusion during accretion of Kolyma-Omolon } \\
\text { superterrane to northeastern margin of the North Asian } \\
\text { Craton. }\end{array}$ \\
\hline $\begin{array}{l}\text { Polousny } \\
(\mathrm{PO})\end{array}$ & $\begin{array}{l}\text { Cassiterite-sulfide-silicate vein } \\
\text { and stockwork deposits } \\
\text { (Ulakhan-Sala); } \\
\text { Polymetallic Pb- } \mathrm{Zn} \pm \mathrm{Cu}( \pm \mathrm{Ag} \text {, } \\
\mathrm{Au} \text { ) vein and stockwork } \\
\text { deposits (Aragochan, Dalnee) }\end{array}$ & $\begin{array}{l}\text { Russia, East- } \\
\text { Central Yakutia } \\
\text { (Verkhoyansk } \\
\text { area) }\end{array}$ & $\begin{array}{l}\text { Granitoids related to } \\
\text { Northern granite belt (too } \\
\text { small to show at } 10 \mathrm{M} \\
\text { scale) }\end{array}$ & $\begin{array}{l}\text { Interpreted as Middle Cretaceous } \\
\text { (Neocomian to Aptian). }{ }^{40} \mathrm{Ar}-{ }^{39} \mathrm{Ar} \\
\text { isotopic age of } 120 \text { to } 130 \mathrm{Ma} \text {. }\end{array}$ & $\begin{array}{l}\text { Belt interpreted as forming during collision of the } \\
\text { Kolyma-Omolon superterrane and the North Asian } \\
\text { Craton and associated regional metamorphism and } \\
\text { generation of anatectic granitoids. }\end{array}$ \\
\hline $\begin{array}{l}\text { Yana- } \\
\text { Adycha } \\
\text { (YAd) }\end{array}$ & $\begin{array}{l}\text { Cassiterite-sulfide-silicate vein } \\
\text { and stockwork (Ege-Khaya, } \\
\text { Ilin-Tas, Burgochan); } \\
\text { Sn-W greisen, stockwork, and } \\
\text { quartz vein (Kester) }\end{array}$ & $\begin{array}{l}\text { Russia, East- } \\
\text { Central Yakutia } \\
\text { (Verkhoyansk } \\
\text { area) }\end{array}$ & $\begin{array}{l}\text { Replacements in } \\
\text { Transverse granite belt } \\
\text { (too small to show at } 10 \mathrm{M} \\
\text { scale) }\end{array}$ & Interpreted as mid-Cretaceous. & $\begin{array}{l}\text { Belt interpreted as forming during collision of the } \\
\text { Kolyma-Omolon superterrane and the North Asian } \\
\text { Craton and associated regional metamorphism and } \\
\text { generation of anatectic granitoids. }\end{array}$ \\
\hline $\begin{array}{l}\text { Tompo } \\
\text { (TO) }\end{array}$ & $\begin{array}{l}\mathrm{W} \pm \mathrm{Mo} \pm \text { Be skarn (Agylky); } \\
\text { Sn-W greisen, stockwork, and } \\
\text { quartz vein (Erikag, } \\
\text { Dzhuptagan) }\end{array}$ & $\begin{array}{l}\text { Russia, East- } \\
\text { Central Yakutia } \\
\text { (Verkhoyansk } \\
\text { area) }\end{array}$ & $\begin{array}{l}\text { Replacements in } \\
\text { Transverse granite belt } \\
\text { (too small to show at } 10 \mathrm{M} \\
\text { scale) }\end{array}$ & Interpreted as Neocomian. & $\begin{array}{l}\text { Belt interpreted as forming during collision of the } \\
\text { Kolyma-Omolon superterrane and the North Asian } \\
\text { Craton and associated regional metamorphism and } \\
\text { generation of anatectic granitoids. Belt occurs along } \\
\text { sublatitudinal high-angle, probable strike-slip faults that } \\
\text { cut Permian to Middle Jurassic sandstone and shale. }\end{array}$ \\
\hline
\end{tabular}




\begin{tabular}{|c|c|c|c|c|c|}
\hline $\begin{array}{l}\text { Name } \\
\text { (Symbol) }\end{array}$ & $\begin{array}{l}\text { Mineral Deposit Models } \\
\text { (Major Deposits) }\end{array}$ & Country, Region & $\begin{array}{l}\text { Unit or Strucutre } \\
\text { Related to Origin of } \\
\text { Metallogenic Belt }\end{array}$ & Age Range of Metallogenic Belt & $\begin{array}{l}\text { Tectonic Event for Origin of Metallogenic Belt. } \\
\text { Comments }\end{array}$ \\
\hline $\begin{array}{l}\text { Kondyor- } \\
\text { Feklistov } \\
\text { (KDF) }\end{array}$ & $\begin{array}{l}\text { Zoned mafic-ultramafic Cr- } \\
\text { PGE (Kondyor) }\end{array}$ & Russia, Far East & $\begin{array}{l}\text { Mafic-ultramafic } \\
\text { intrusions (too small to } \\
\text { show at } 10 \mathrm{M} \text { scale) } \\
\text { intruded along major fault } \\
\text { cuitting North Asian } \\
\text { Craton and northeastern } \\
\text { part of Tukuringra- } \\
\text { Dzhagdy terrane }\end{array}$ & $\begin{array}{l}\text { Early Cretaceous. K-Ar isotopic } \\
\text { ages for the zoned mafic- } \\
\text { ultramafic intrusions in the } \\
\text { Kondyor metallogenic belt range } \\
\text { from } 110 \text { to } 160 \mathrm{Ma} .{ }^{40} \mathrm{Ar}-{ }^{39} \mathrm{Ar} \\
\text { isotopic age of } 127 \mathrm{Ma} \text { recently } \\
\text { obtained for the alkalic mafic and } \\
\text { ultramafic igneous rocks at } \\
\text { Ingagli. }\end{array}$ & $\begin{array}{l}\text { Belt interpreted as forming during intrusion of mafic- } \\
\text { ultramafic plutons along a deep-seated fault that formed } \\
\text { along the North Asian Craton margin during collision } \\
\text { and accretion of outboard terranes. }\end{array}$ \\
\hline $\begin{array}{l}\text { Mavrinsk } \\
(\mathrm{MV})\end{array}$ & $\begin{array}{l}\text { Clastic sediment-hosted } \mathrm{Hg} \pm \mathrm{Sb} \\
\text { (Mavrinskoye, Orlinogorskoye) }\end{array}$ & $\begin{array}{l}\text { Russia, Southern- } \\
\text { Eastern Siberia } \\
\text { (Salair Range) }\end{array}$ & $\begin{array}{l}\text { Replacements along major } \\
\text { fault between Salair } \\
\text { terrane and Kuznetsk } \\
\text { orogenic basin }\end{array}$ & $\begin{array}{l}\text { Early Jurassic or younger. K-Ar } \\
\text { isotopic age of } 190 \text { to } 200 \mathrm{Ma} \text { for } \\
\text { lamprophyre dikes provides a } \\
\text { minimum age for } \mathrm{Hg} \text { deposits. }\end{array}$ & $\begin{array}{l}\text { Belt interpreted as forming during intraplate rifting and } \\
\text { interblock strike-slip faulting during the Late Paleozoic } \\
\text { to Early Mesozoic. }\end{array}$ \\
\hline $\begin{array}{l}\text { Kuznetsk } \\
(\mathrm{KE})\end{array}$ & $\begin{array}{l}\text { Volcanic-hosted Hg } \\
\text { (Kupriyanovskoye, Belo- } \\
\text { Osipovskoye); } \\
\text { Carbonate-hosted Hg (Pezass) }\end{array}$ & $\begin{array}{l}\text { Russia, Eastern } \\
\text { Siberia }\end{array}$ & $\begin{array}{l}\text { Replacements along major } \\
\text { faults cutting Kuznetsk } \\
\text { orogenic basin, Altai } \\
\text { volcanic-plutonic belt and } \\
\text { Telbes-Kitat island-arc } \\
\text { terrane. }\end{array}$ & Middle to Late Jurassic. & $\begin{array}{l}\text { Belt interpreted as forming during intraplate rifting and } \\
\text { interblock strike-slip faulting during the Late Paleozoic } \\
\text { to Early Mesozoic. Belt occurs along the major } \\
\text { Kuznetsk fault. }\end{array}$ \\
\hline $\begin{array}{l}\text { Sistigkhem } \\
\text { (SS) }\end{array}$ & $\begin{array}{l}\text { Carbonate-hosted Hg-Sb } \\
\text { (Kukshinskoye, Oktyabrskoye) }\end{array}$ & $\begin{array}{l}\text { Russia, Southern- } \\
\text { Eastern Siberia } \\
\text { (Tuva area) }\end{array}$ & $\begin{array}{l}\text { Replacements along and } \\
\text { adjacent to Khemchic- } \\
\text { Kurtushiba fault and } \\
\text { conjugate faults that } \\
\text { bound the Kurtushiba and } \\
\text { Alambai terranes. }\end{array}$ & $\begin{array}{l}\text { Interpreted as Middle to Late } \\
\text { Jurassic. }\end{array}$ & $\begin{array}{l}\text { Belt is interpreted as related to magmatism along } \\
\text { transextension zones along transform micro plate } \\
\text { boundaries and within plate (plume) environment. }\end{array}$ \\
\hline $\begin{array}{l}\text { Eravninsky } \\
\text { (Era) }\end{array}$ & $\begin{array}{l}\text { Cassiterite-sulfide-silicate vein } \\
\text { and stockwork } \\
\text { (Kydzhimitskoye); } \\
\text { Carbonate-hosted fluorspar } \\
\text { (Egitinskoye) }\end{array}$ & $\begin{array}{l}\text { Russia, Western } \\
\text { Transbaikalia }\end{array}$ & $\begin{array}{l}\text { Replacements, volcanic } \\
\text { complexes related to } \\
\text { Trans-Baikalian- } \\
\text { Daxinganling (trbv) } \\
\text { sedimentary-volcanic- } \\
\text { plutonic belt that intrudes } \\
\text { and overlaps the Orhon- } \\
\text { Ikatsky terrane, Barguzin- } \\
\text { Vitim granitoid belt, and } \\
\text { Selenga sedimentary- } \\
\text { volcanic plutonic belt }\end{array}$ & $\begin{array}{l}\text { Interpreted as Middle Jurassic to } \\
\text { Early Cretaceous. }\end{array}$ & $\begin{array}{l}\text { Belt is interpreted as related to magmatism along } \\
\text { transextension zones along transform micro plate } \\
\text { boundaries and within plate (plume) environment. }\end{array}$ \\
\hline
\end{tabular}




\begin{tabular}{|c|c|c|c|c|c|}
\hline $\begin{array}{l}\text { Name } \\
\text { (Symbol) }\end{array}$ & $\begin{array}{l}\text { Mineral Deposit Models } \\
\text { (Major Deposits) }\end{array}$ & Country, Region & $\begin{array}{l}\text { Unit or Strucutre } \\
\text { Related to Origin of } \\
\text { Metallogenic Belt }\end{array}$ & Age Range of Metallogenic Belt & $\begin{array}{l}\text { Tectonic Event for Origin of Metallogenic Belt. } \\
\text { Comments }\end{array}$ \\
\hline $\begin{array}{l}\text { Nerchinsky } \\
\text { (Ner) }\end{array}$ & $\begin{array}{l}\text { Granitoid-related Au vein } \\
\text { (Darasunskoye); } \\
\text { W-Mo-Be greisen, stockwork, } \\
\text { and quartz vein } \\
\text { (Muoklakanskoye); } \\
\text { Fluorspar vein (Usuglinskoye) }\end{array}$ & $\begin{array}{l}\text { Russia, Eastern } \\
\text { Transbaikalia }\end{array}$ & $\begin{array}{l}\text { Granitoids and } \\
\text { replacements related to } \\
\text { Trans-Baikalian- } \\
\text { Daxinganling } \\
\text { sedimentary-volcanic- } \\
\text { plutonic belt intruding and } \\
\text { overlapping West } \\
\text { Stanovoy terrane, } \\
\text { Barguzin-Vitim granitoid } \\
\text { belt, and Selenga } \\
\text { sedimentary-volcanic } \\
\text { plutonic belt }\end{array}$ & $\begin{array}{l}\text { Interpreted as Middle Jurassic to } \\
\text { Early Cretaceous. }\end{array}$ & $\begin{array}{l}\text { Belt is interpreted as related to magmatism along } \\
\text { transextension zones along transform micro plate } \\
\text { boundaries and within plate (plume) environment. The } \\
\text { belt is related to granitoids in the Trans-Baikalian- } \\
\text { Daxinganling sedimentary-volcanic-plutonic belt. }\end{array}$ \\
\hline $\begin{array}{l}\text { Shilkinsko- } \\
\text { Tukuringrs } \\
\text { kiy } \\
(\mathrm{ShT})\end{array}$ & $\begin{array}{l}\text { Granitoid-related Au vein } \\
\text { (Ukonikskoe); } \\
\text { Porphyry Au; Au skarn; Au-Ag } \\
\text { epithermal vein; } \\
\text { Porphyry Mo ( } \pm \mathrm{W}, \mathrm{Bi}) \\
\text { (Zhirekenskoye) } \\
\text { W-Mo-Be greisen, stockwork, } \\
\text { and quartz vein; } \\
\text { Cassiterite-sulfide-silicate vein } \\
\text { and stockwork; } \\
\text { Ta-Nb-REE alkaline } \\
\text { metasomatite; } \\
\text { Polymetallic Pb-Zn } \pm \mathrm{Cu}( \pm \mathrm{Ag} \text {, } \\
\text { Au) vein and stockwork } \\
\text { (Berezitovoe ); Au-Ag } \\
\text { epithermal vein (Baleyskoe); } \\
\text { Fluorite vein (Kalanguyskoye) }\end{array}$ & $\begin{array}{l}\text { Russia, Eastern } \\
\text { Transbaikalia }\end{array}$ & $\begin{array}{l}\text { Granitoids, volcanic rocks, } \\
\text { and replacements related } \\
\text { to Trans-Baikalian- } \\
\text { Daxinganling } \\
\text { sedimentary-volcanic- } \\
\text { plutonic belt (too small to } \\
\text { show at } 10 \mathrm{M} \text { scale) } \\
\text { intruding and overlapping } \\
\text { West Stanovoy terrane, } \\
\text { Ononsky terrane, } \\
\text { Argunsky terrane, and } \\
\text { adjacent units. }\end{array}$ & $\begin{array}{l}\text { Interpreted as Middle Jurassic to } \\
\text { Early Cretaceous. }\end{array}$ & $\begin{array}{l}\text { Belt is interpreted as related to magmatism along } \\
\text { transextension zones along transform micro plate } \\
\text { boundaries and within plate (plume) environment. Belt } \\
\text { occurs in basins with continental sedimentary rocks and } \\
\text { alkaline magmatic plutonic and volcanic rocks that } \\
\text { occur along the Mongol-Okhotsk suture that separates } \\
\text { various terranes and the North Asian Craton and the } \\
\text { Sino-Korean Craton. }\end{array}$ \\
\hline
\end{tabular}




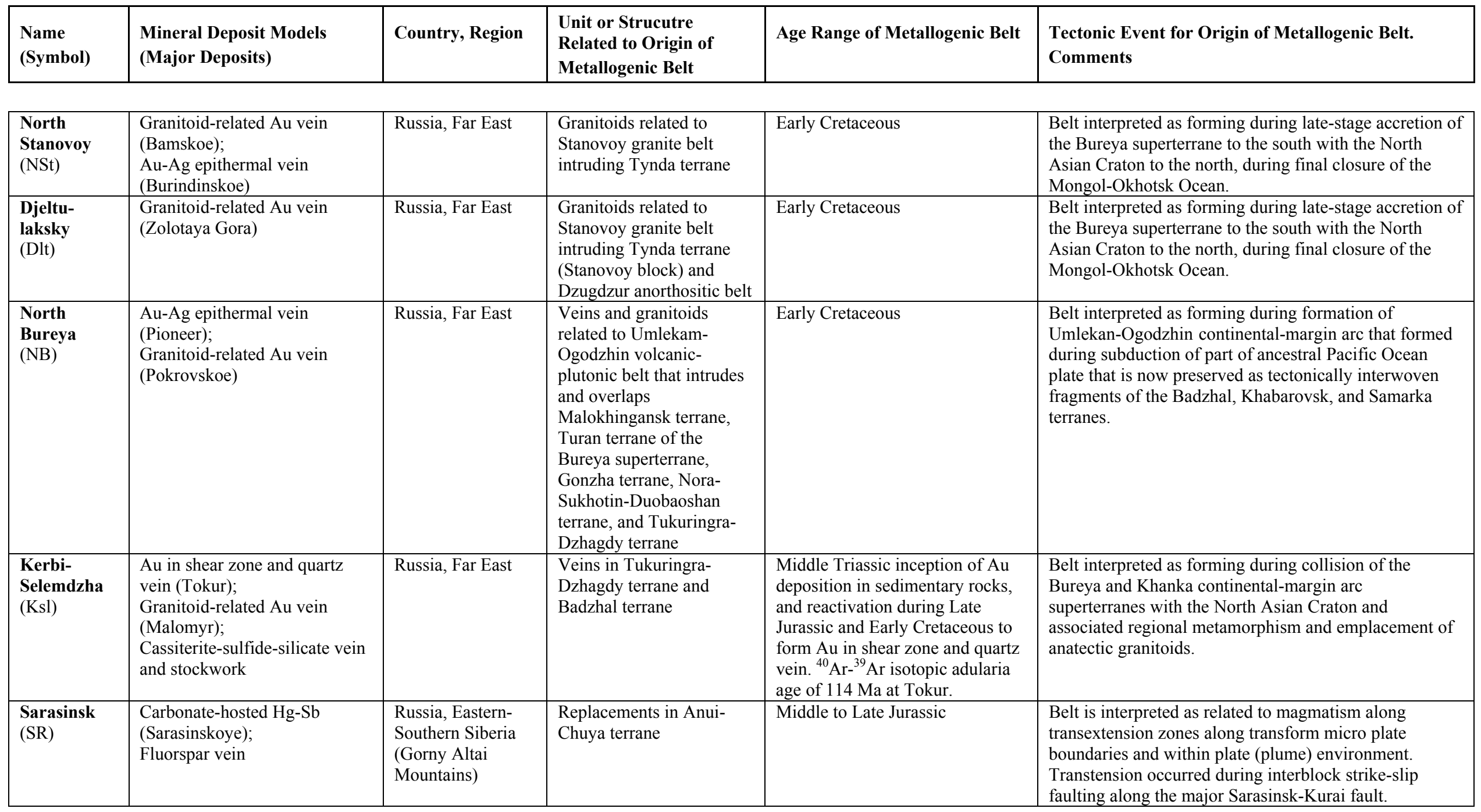




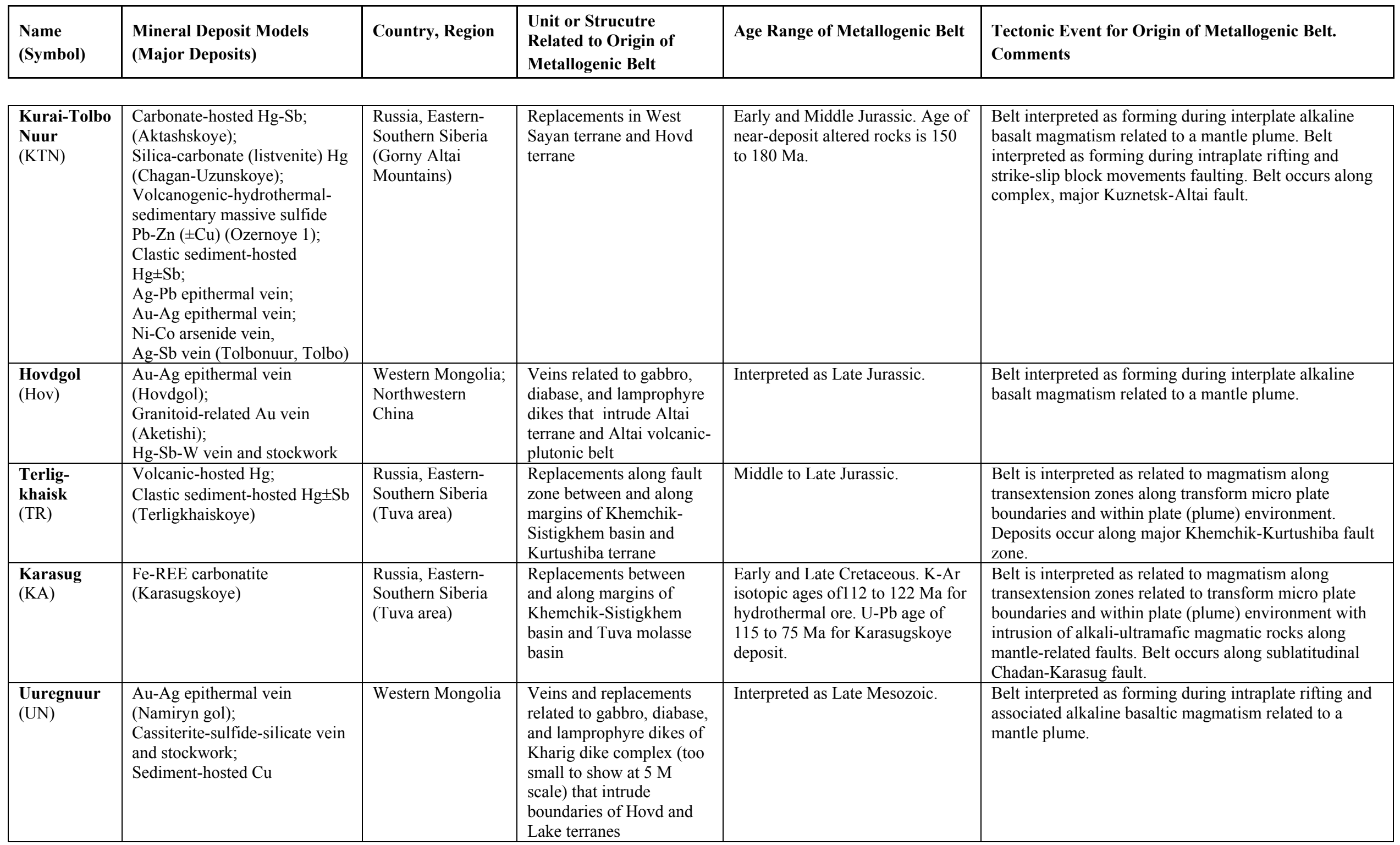




\begin{tabular}{|c|c|c|c|c|c|}
\hline $\begin{array}{l}\text { Name } \\
\text { (Symbol) }\end{array}$ & $\begin{array}{l}\text { Mineral Deposit Models } \\
\text { (Major Deposits) }\end{array}$ & Country, Region & $\begin{array}{l}\text { Unit or Strucutre } \\
\text { Related to Origin of } \\
\text { Metallogenic Belt }\end{array}$ & Age Range of Metallogenic Belt & $\begin{array}{l}\text { Tectonic Event for Origin of Metallogenic Belt. } \\
\text { Comments }\end{array}$ \\
\hline $\begin{array}{l}\text { Khilokskiy } \\
\text { (Khl) }\end{array}$ & $\begin{array}{l}\text { Sn-W greisen, stockwork, and } \\
\text { quartz vein (Bom- } \\
\text { Gorkhonskoye) }\end{array}$ & $\begin{array}{l}\text { Russia, Western } \\
\text { Transbaikalia }\end{array}$ & $\begin{array}{l}\text { Veins, replacements, } \\
\text { granitoids, volcanic } \\
\text { complexes related to } \\
\text { Trans-Baikalian- } \\
\text { Daxinganling } \\
\text { sedimentary-volcanic- } \\
\text { plutonic belt that overlies } \\
\text { and intrudes Barguzin- } \\
\text { Vitim granitoid belt and } \\
\text { Selenga sedimentary- } \\
\text { volcanic plutonic belt. }\end{array}$ & $\begin{array}{l}\text { Interpreted as Middle Jurassic to } \\
\text { Early Cretaceous. }\end{array}$ & $\begin{array}{l}\text { Belt is interpreted as related to magmatism that occurred } \\
\text { transpression zones related to transform micro plate } \\
\text { boundaries and within plate (plume) environment. }\end{array}$ \\
\hline $\begin{array}{l}\text { Onon- } \\
\text { Chikoiskiy } \\
(\mathrm{OCH})\end{array}$ & $\begin{array}{l}\text { Sn-W greisen, stockwork, and } \\
\text { quartz vein (Shumilovskoye); } \\
\text { W-Mo-Be greisen, stockwork, } \\
\text { and quartz vein (Upper Kumyr) }\end{array}$ & $\begin{array}{l}\text { Russia, Eastern } \\
\text { Transbaikalia }\end{array}$ & $\begin{array}{l}\text { Veins, replacements, } \\
\text { volcanic complexes and } \\
\text { granitoids related to } \\
\text { Trans-Baikalian- } \\
\text { Daxinganling (trbv) } \\
\text { sedimentary-volcanic- } \\
\text { plutonic belt that overlies } \\
\text { and intrudes Hangay- } \\
\text { Dauria terrane, Zag-Haraa } \\
\text { turbidite basin, and } \\
\text { Selenga sedimentary- } \\
\text { volcanic plutonic belt. }\end{array}$ & $\begin{array}{l}\text { Interpreted as Middle Jurassic to } \\
\text { Early Cretaceous. }\end{array}$ & $\begin{array}{l}\text { Belt interpreted as related to magmatism that occurred } \\
\text { transpression zones related to transform micro plate } \\
\text { boundaries and within plate (plume) environment. }\end{array}$ \\
\hline
\end{tabular}




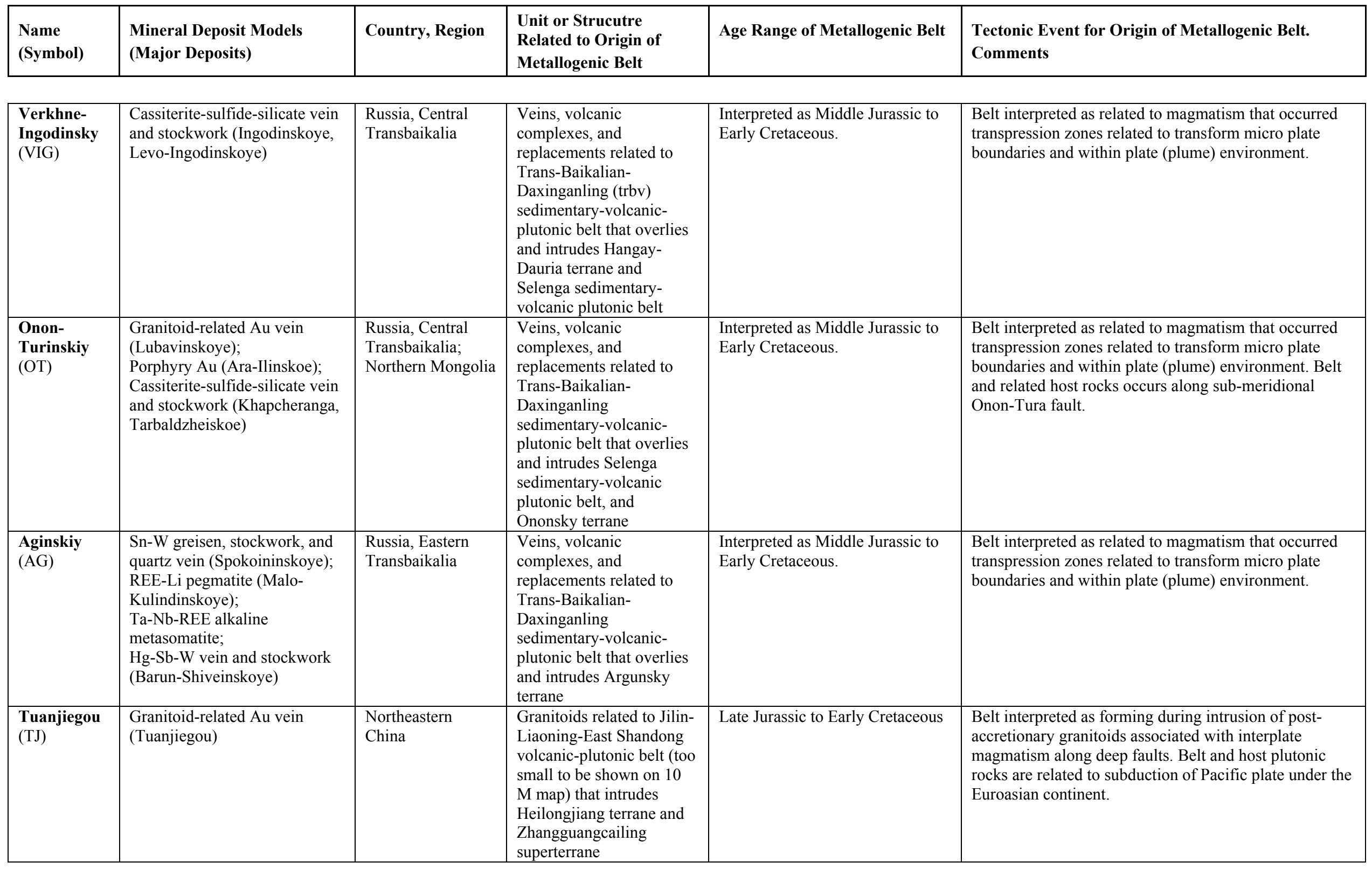




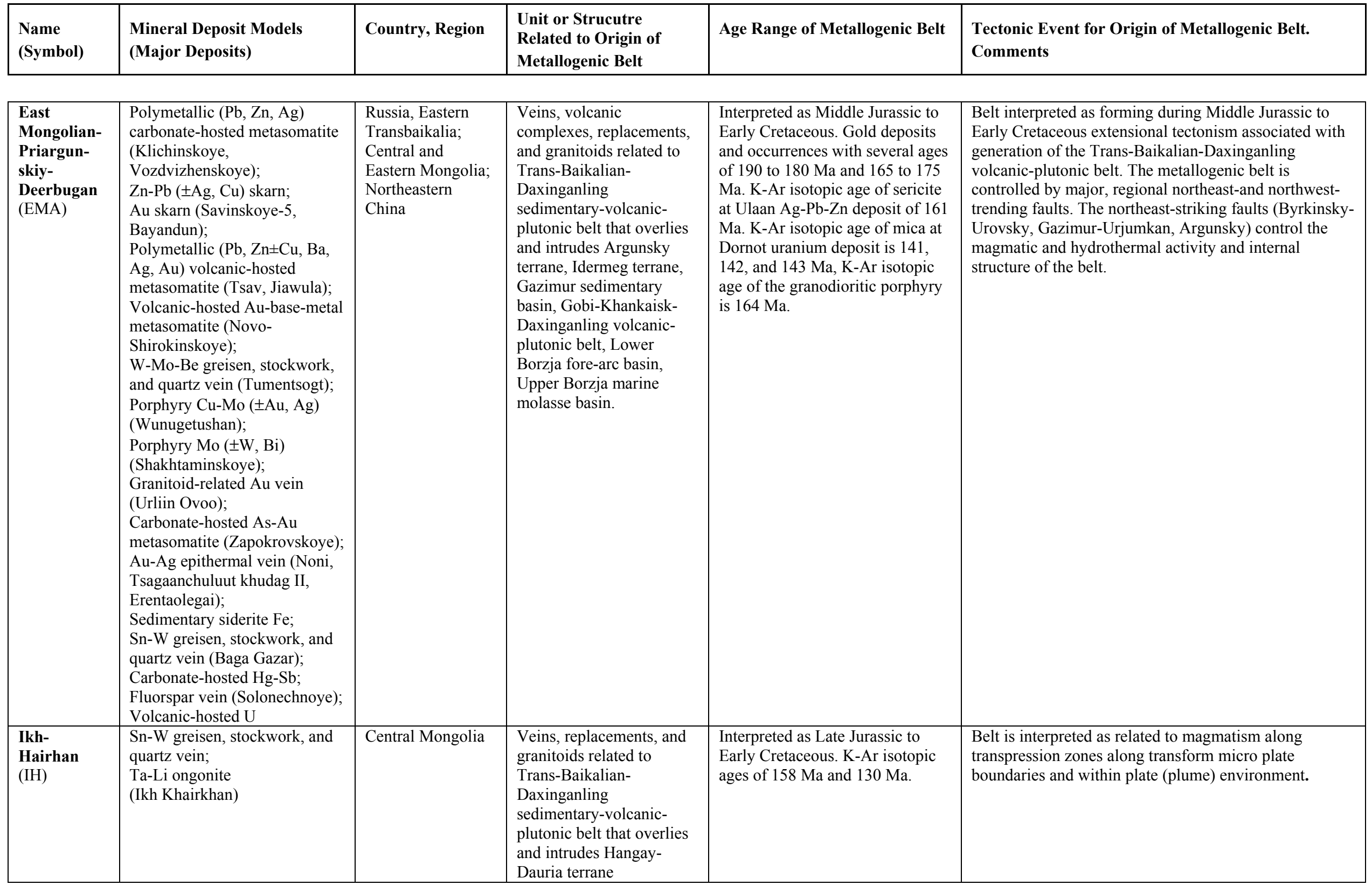




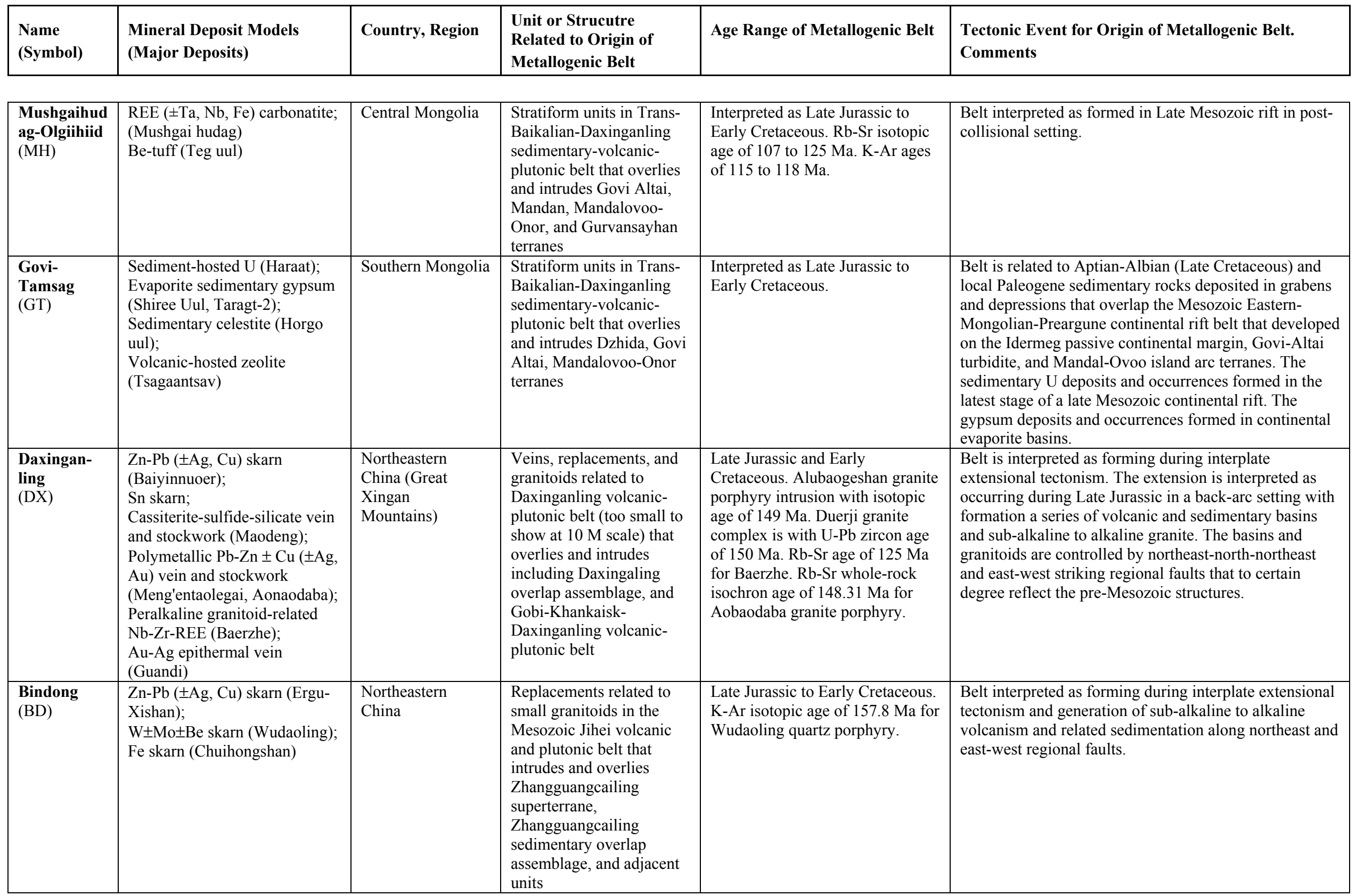




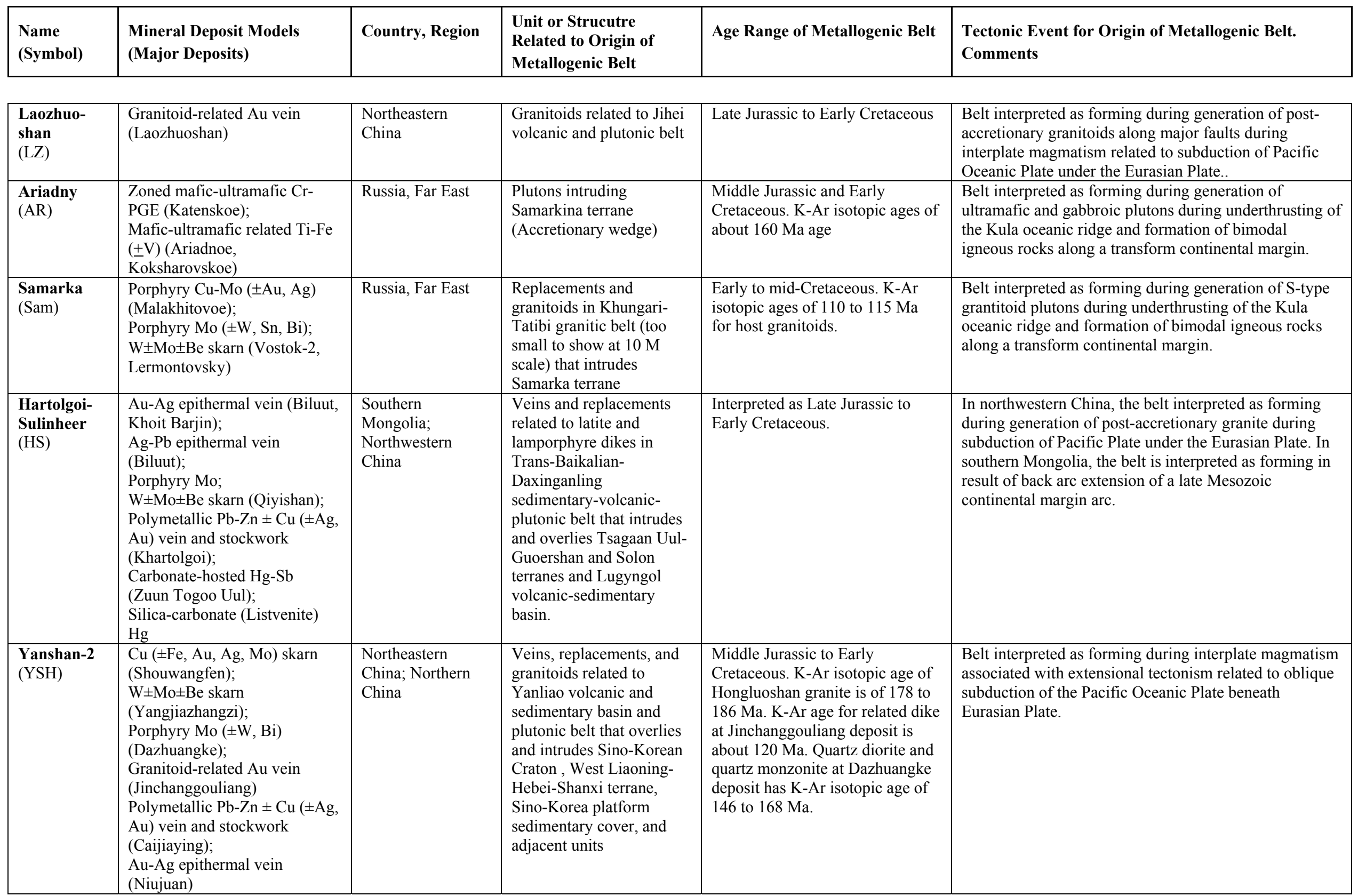




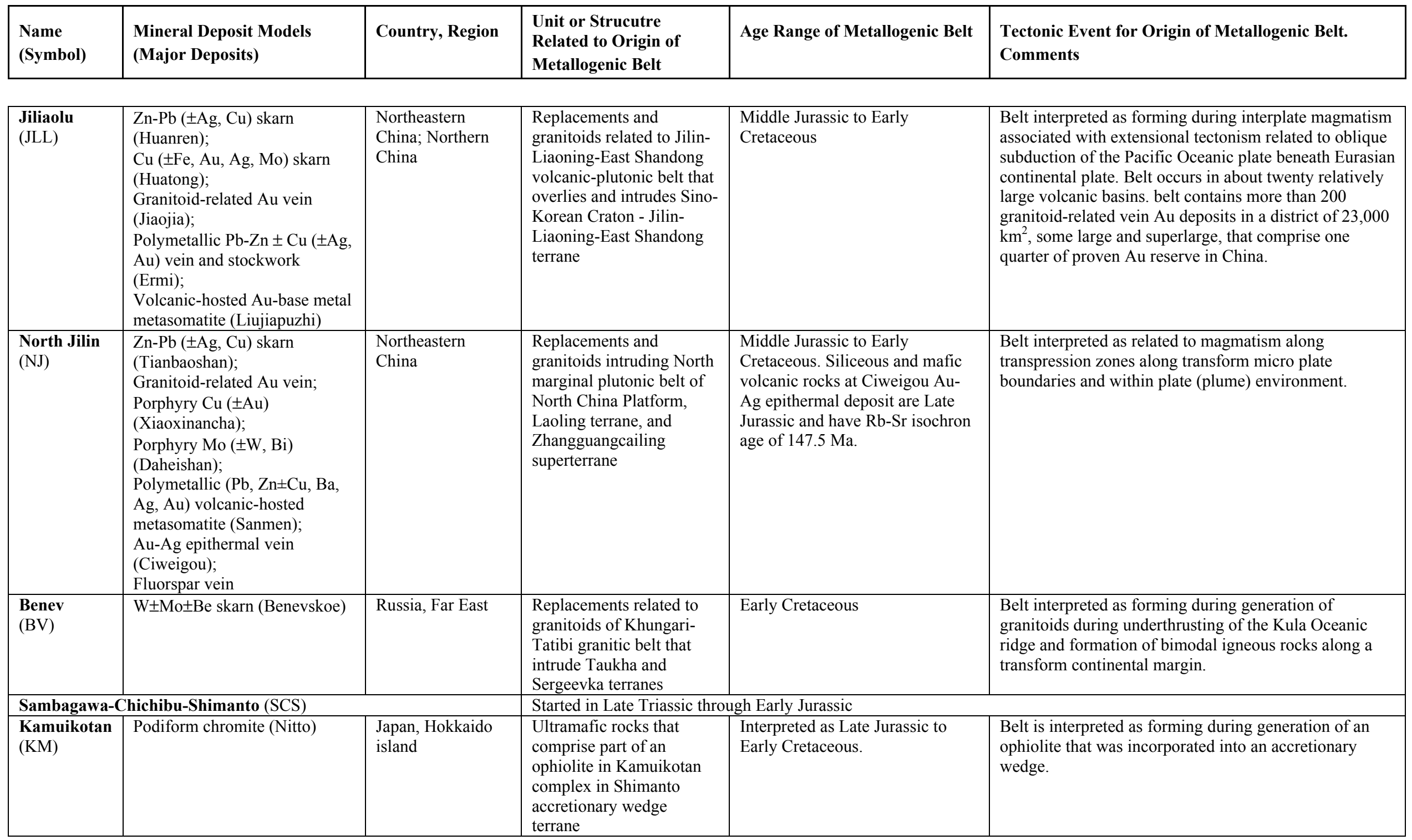




\begin{tabular}{|c|c|c|c|c|c|}
\hline $\begin{array}{l}\text { Name } \\
\text { (Symbol) }\end{array}$ & $\begin{array}{l}\text { Mineral Deposit Models } \\
\text { (Major Deposits) }\end{array}$ & Country, Region & $\begin{array}{l}\text { Unit or Strucutre } \\
\text { Related to Origin of } \\
\text { Metallogenic Belt }\end{array}$ & Age Range of Metallogenic Belt & $\begin{array}{l}\text { Tectonic Event for Origin of Metallogenic Belt. } \\
\text { Comments }\end{array}$ \\
\hline $\begin{array}{l}\text { Laiwu } \\
(\mathrm{LW})\end{array}$ & Fe skarn (Jinling) & Northern China & $\begin{array}{l}\text { Replacements related to } \\
\text { Late Jurassic to Early } \\
\text { Cretaceous granitoids in } \\
\text { Jilin-Liaoning-East } \\
\text { Shandong volcanic- } \\
\text { plutonic belt that intrudes } \\
\text { Sino Korean Craton - } \\
\text { West Liaoning-Hebei- } \\
\text { Shanxi terrane }\end{array}$ & $\begin{array}{l}\text { Late Jurassic to Early Cretaceous. } \\
\text { Isotopic ages of } 110 \text { to } 128 \mathrm{Ma} \text {. }\end{array}$ & $\begin{array}{l}\text { Belt interpreted as forming during granitoid plutonism } \\
\text { associated with extensional faults related to subduction } \\
\text { of Pacific Plate under Eurasian Plate. Host granitoids of } \\
\text { the Jinling Complex are interpreted as forming during } \\
\text { the Yanshan orogeny. }\end{array}$ \\
\hline $\begin{array}{l}\text { Taebaegsan } \\
\text { (Tae) }\end{array}$ & $\begin{array}{l}\text { Fe skarn (Kangwon, Dongnam, } \\
\text { Susuk); } \\
\text { Fe-Zn skarn (Yomisan); Zn-Pb } \\
\text { (Ag, Cu, W) skarn; } \\
\mathrm{W} \pm \mathrm{Mo} \pm \mathrm{Be} \text { skarn (Wondong, } \\
\text { Sangdong); REE-Li pegmatite; } \\
\text { Au in shear zone and quartz } \\
\text { vein (Seojom) }\end{array}$ & South Korea & $\begin{array}{l}\text { Replacements and dikes } \\
\text { related to Middle Jurassic } \\
\text { through Early Cretaceous } \\
\text { granitoids in Daebu } \\
\text { Granite intruding } \\
\text { Yeongnam Meta Complex } \\
\text { and Great Limestone } \\
\text { Group, (too small to show } \\
\text { at } 10 \mathrm{M} \text { scale). }\end{array}$ & $\begin{array}{l}\text { Interpreted as Middle Jurassic } \\
\text { through Early Cretaceous. }\end{array}$ & $\begin{array}{l}\text { Belt interpreted as forming during intrusion of } \\
\text { granitoids associated with Late Jurassic to Early } \\
\text { Cretaceous Daebo granite that intruded during the } \\
\text { Daebo orogeny. Granite consists of biotite granite, } \\
\text { feldspar porphyry, and granite porphyry that intrude } \\
\text { Precambrian metasedimentary rocks. Deposits formed } \\
\text { during contact metasomatism of calcareous layers in } \\
\text { metasedimentary rock. }\end{array}$ \\
\hline $\begin{array}{l}\text { Kitakami } \\
\text { (Kit) }\end{array}$ & $\begin{array}{l}\mathrm{Cu}( \pm \mathrm{Fe}, \mathrm{Au}, \mathrm{Ag}, \mathrm{Mo}) \text { skarn } \\
\text { (Kamaishi); } \\
\text { Granitoid-related } \mathrm{Au} \text { vein } \\
\text { (Oya) }\end{array}$ & Japan & $\begin{array}{l}\text { Replacements in Early } \\
\text { Cretaceous Hiroshima } \\
\text { granitic belt (too small to } \\
\text { show at } 10 \mathrm{M} \text { scale) } \\
\text { intruding South Kitakami } \\
\text { and Mino-Tamba- } \\
\text { Chichibu terranes. }\end{array}$ & $\begin{array}{l}\text { Early Cretaceous (Aptian through } \\
\text { Albian). K-Ar isotopic ages of } \\
120 \mathrm{Ma} \text { to } 110 \mathrm{Ma} \text { for deposit- } \\
\text { related granitic rocks in the } \\
\text { Kitakami Mountains. }\end{array}$ & $\begin{array}{l}\text { Belt interpreted as forming during intrusion of } \\
\text { granitoids associated with a continental-margin arc and } \\
\text { siliceous magmatism. }\end{array}$ \\
\hline $\begin{array}{l}\text { Chokhchur- } \\
\text { Chekur- } \\
\text { dakh } \\
\text { (CC) }\end{array}$ & $\begin{array}{l}\text { Cassiterite-sulfide-silicate vein } \\
\text { and stockwork (Churpunya, } \\
\text { Chokurdakh) }\end{array}$ & $\begin{array}{l}\text { Russia, East- } \\
\text { Central Yakutia } \\
\text { (Verkhoyansk } \\
\text { area) }\end{array}$ & $\begin{array}{l}\text { Veins and replacements in } \\
\text { Svyatoi Nos volcanic belt } \\
\text { (too small to show at } 10 \mathrm{M} \\
\text { scale) }\end{array}$ & $\begin{array}{l}\text { Interpreted as Aptian to Late } \\
\text { Cretaceous. The granitoids are } \\
\text { dated by }{ }^{40} \mathrm{Ar}-{ }^{39} \mathrm{Ar} \text { at } 105 \text { to } 106 \\
\text { Ma. }\end{array}$ & $\begin{array}{l}\text { Belt interpreted as forming during post-accretionary } \\
\text { extension related to initiation of opening of Eurasia } \\
\text { Basin. Belt occurs along the Yana fault. Belt hosted in } \\
\text { granodiorite, amphibole-biotite granite, and subalkali } \\
\text { granite that form part of Svyatoy Nos magmatic arc. }\end{array}$ \\
\hline
\end{tabular}




\begin{tabular}{|c|c|c|c|c|c|}
\hline $\begin{array}{l}\text { Name } \\
\text { (Symbol) }\end{array}$ & $\begin{array}{l}\text { Mineral Deposit Models } \\
\text { (Major Deposits) }\end{array}$ & Country, Region & $\begin{array}{l}\text { Unit or Strucutre } \\
\text { Related to Origin of } \\
\text { Metallogenic Belt } \\
\end{array}$ & Age Range of Metallogenic Belt & $\begin{array}{l}\text { Tectonic Event for Origin of Metallogenic Belt. } \\
\text { Comments }\end{array}$ \\
\hline $\begin{array}{l}\text { Eckyuchu- } \\
\text { Billyakh } \\
\text { (EB) }\end{array}$ & $\begin{array}{l}\text { Polymetallic } \mathrm{Pb}-\mathrm{Zn} \pm \mathrm{Cu}( \pm \mathrm{Ag}, \\
\mathrm{Au} \text { ) vein and stockwork } \\
\text { (Prognoz); } \\
\text { Clastic-sediment-hosted Sb- } \\
\mathrm{Au} \text {; Hg-Sb-W vein and } \\
\text { stockwork (Zvyozdochka); } \\
\text { Ag-Sb vein vein; } \\
\text { Au-Ag epithermal vein }\end{array}$ & $\begin{array}{l}\text { Russia, East- } \\
\text { Central Yakutia } \\
\text { (Verkhoyansk } \\
\text { area) }\end{array}$ & $\begin{array}{l}\text { Veins and replacements } \\
\text { related to Transverse } \\
\text { granite belt (too small to } \\
\text { show at } 10 \mathrm{M} \text { scale) that } \\
\text { intrudes North Asian } \\
\text { Craton Margin - } \\
\text { Verkhoyansk fold and } \\
\text { thrust belt }\end{array}$ & $\begin{array}{l}\text { Interpreted as Aptian to Late } \\
\text { Cretaceous. Granitoid stocks and } \\
\text { dikes of various composition are } \\
\text { have }{ }^{40} \mathrm{Ar}-{ }^{39} \mathrm{Ar} \text { isotopic ages of } \\
\text { older than } 120 \text { Ma. Khoboyatu- } \\
\text { Echiy granite pluton has }{ }^{40} \mathrm{Ar}-{ }^{39} \mathrm{Ar} \\
\text { age of } 97 \mathrm{Ma} \text {. }\end{array}$ & $\begin{array}{l}\text { Belt interpreted as forming during post-accretionary } \\
\text { extension related to initiation of opening of Eurasia } \\
\text { Basin. Belt hosted in granitoid stocks and dikes that } \\
\text { occur at the terminations of the Transverse granitoid } \\
\text { belt. }\end{array}$ \\
\hline $\begin{array}{l}\text { Taryn } \\
\text { (Tar) }\end{array}$ & $\begin{array}{l}\text { Clastic-sediment-hosted Sb-Au } \\
\text { (Senatachan, Sarylakh, } \\
\text { Kupol'noe) }\end{array}$ & $\begin{array}{l}\text { Russia, East- } \\
\text { Central Yakutia } \\
\text { (Verkhoyansk } \\
\text { area) }\end{array}$ & $\begin{array}{l}\text { Veins and replacements in } \\
\text { Kular-Nera terrane and } \\
\text { North Asian Craton } \\
\text { Margin - Verkhoyansk } \\
\text { fold and thrust belt }\end{array}$ & $\begin{array}{l}\text { Interpreted as Aptian to Late } \\
\text { Cretaceous. }\end{array}$ & $\begin{array}{l}\text { Belt interpreted as forming during post-accretionary } \\
\text { extension related to initiation of opening of Eurasia } \\
\text { Basin. }\end{array}$ \\
\hline $\begin{array}{l}\text { Selennyakh } \\
\text { (SE) }\end{array}$ & $\begin{array}{l}\text { Carbonate-hosted Hg-Sb (Gal } \\
\text { Khaya, Pologoye, Arbat); } \\
\text { Volcanic-hosted Hg (Dogdo); } \\
\text { Ag-Sb vein (Kysylga) }\end{array}$ & $\begin{array}{l}\text { Russia, East- } \\
\text { Central Yakutia } \\
\text { (Verkhoyansk } \\
\text { area) }\end{array}$ & $\begin{array}{l}\text { Veins and replacements } \\
\text { related to Uyandina- } \\
\text { Yasachnaya volcanic belt } \\
\text { that overlies Omulevka } \\
\text { passive continental margin } \\
\text { terrane of Kolyma- } \\
\text { Omolon superterrane, and } \\
\text { adjacent terranes. }\end{array}$ & $\begin{array}{l}\text { Interpreted as Aptian to Late } \\
\text { Cretaceous. }\end{array}$ & $\begin{array}{l}\text { Belt interpreted as forming during post-accretionary } \\
\text { extension related to initiation of opening of Eurasia } \\
\text { Basin. }\end{array}$ \\
\hline $\begin{array}{l}\text { Khandyga } \\
\text { (Kha) }\end{array}$ & $\begin{array}{l}\text { Ag-Sb vein; } \\
\text { Carbonate-hosted As-Au } \\
\text { metasomatite; } \\
\text { Clastic-sediment-hosted } \mathrm{Sb}-\mathrm{Au} \\
\text { (Senduchen); } \\
\text { Clastic sediment-hosted } \mathrm{Hg} \pm \mathrm{Sb} \\
\text { (Seikimyan) }\end{array}$ & $\begin{array}{l}\text { Russia, East- } \\
\text { Central Yakutia } \\
\text { (Verkhoyansk } \\
\text { area) }\end{array}$ & $\begin{array}{l}\text { Veins and replacements in } \\
\text { North Asian Craton } \\
\text { Margin - Verkhoyansk } \\
\text { fold and thrust belt }\end{array}$ & $\begin{array}{l}\text { Interpreted as Aptian to Late } \\
\text { Cretaceous. }\end{array}$ & $\begin{array}{l}\text { Belt interpreted as forming during post-accretionary } \\
\text { extension related to initiation of opening of Eurasia } \\
\text { Basin. Belt occurs in veins and replacements in the } \\
\text { southern Verkhoyansk fold and thrust alon the Sette- } \\
\text { Daban tectonic zone. }\end{array}$ \\
\hline
\end{tabular}




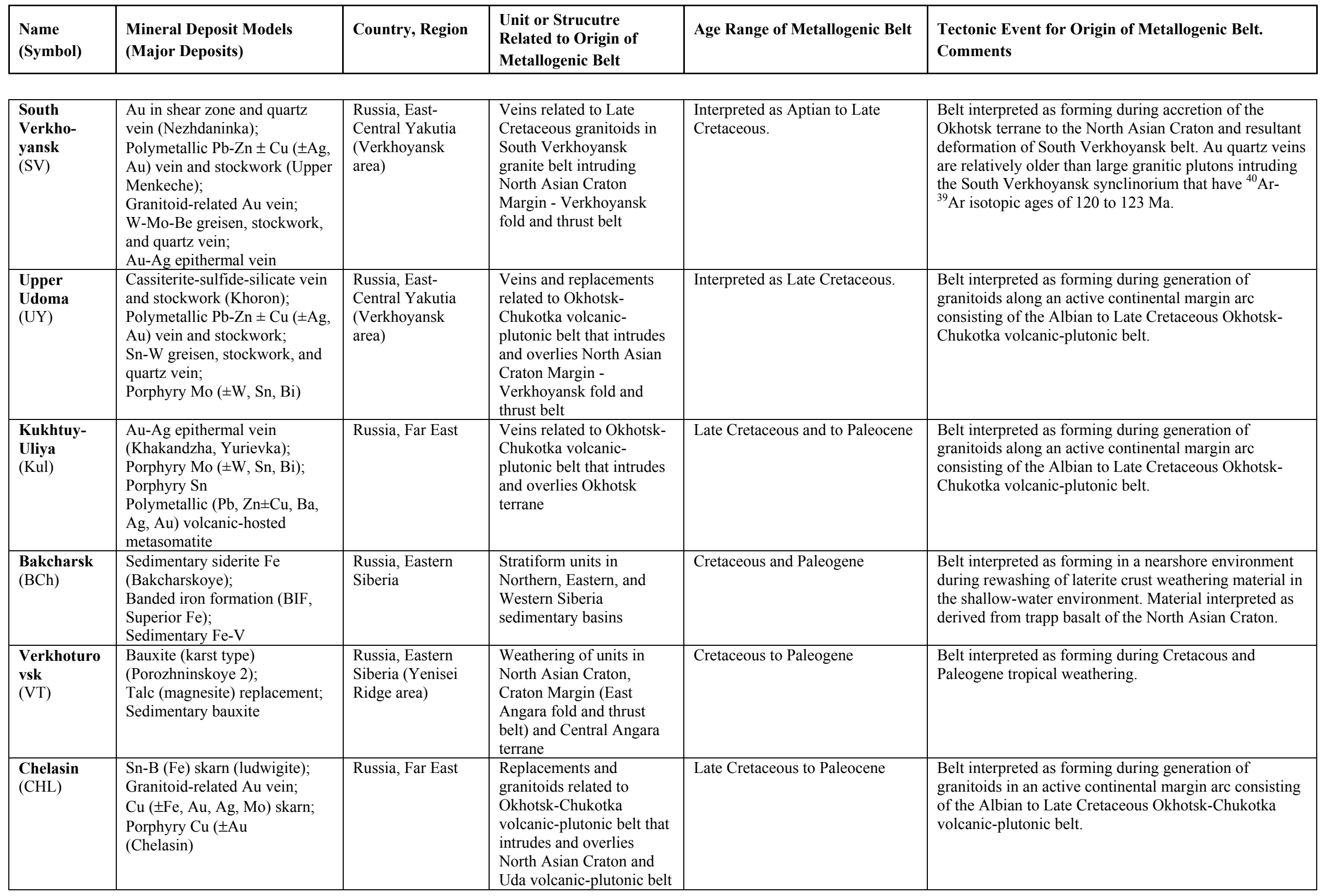




\begin{tabular}{|c|c|c|c|c|c|}
\hline $\begin{array}{l}\text { Name } \\
\text { (Symbol) }\end{array}$ & $\begin{array}{l}\text { Mineral Deposit Models } \\
\text { (Major Deposits) }\end{array}$ & Country, Region & $\begin{array}{l}\text { Unit or Strucutre } \\
\text { Related to Origin of } \\
\text { Metallogenic Belt }\end{array}$ & Age Range of Metallogenic Belt & $\begin{array}{l}\text { Tectonic Event for Origin of Metallogenic Belt. } \\
\text { Comments }\end{array}$ \\
\hline $\begin{array}{l}\text { Belininsk } \\
\text { (Bel) }\end{array}$ & $\begin{array}{l}\text { Bauxite (karst type) } \\
\text { (Barzasskoye); } \\
\text { Lateritic Ni (Belininskoye); } \\
\text { Sedimentary bauxite; } \\
\text { Talc (magnesite) replacement }\end{array}$ & $\begin{array}{l}\text { Russia, Southern- } \\
\text { Eastern Siberia } \\
\text { (Salair Range, } \\
\text { Kuznetsk Alatau } \\
\text { Mountains) }\end{array}$ & $\begin{array}{l}\text { Weathering of } \\
\text { sedimentary rocks in } \\
\text { Salair and Telbes-Kitat } \\
\text { island-arc terranes, and } \\
\text { ultramafic-mafic bodies in } \\
\text { Alambai accretionary } \\
\text { wedge terrane }\end{array}$ & Late Cretaceous to Paleocene & $\begin{array}{l}\text { Belt interpreted as forming as deuterogenic laterite } \\
\text { derived from argillic karst material. Lateritic Ni deposits } \\
\text { formed from of weathering crusts on serpentinized } \\
\text { ultramafic rocks. }\end{array}$ \\
\hline $\begin{array}{l}\text { Djotsk } \\
\text { (DJ) }\end{array}$ & $\begin{array}{l}\text { Weathering crust } \mathrm{Mn}( \pm \mathrm{Fe}) \\
\text { (Seibinskoye) }\end{array}$ & $\begin{array}{l}\text { Russia, Eastern } \\
\text { Siberia }\end{array}$ & $\begin{array}{l}\text { Weathering of } \\
\text { sedimentary rocks in } \\
\text { Kizir-Kazir terrane (Island } \\
\text { arc) (too small to show at } \\
10 \mathrm{M} \text { scale) }\end{array}$ & Late Cretaceous to Paleogene & $\begin{array}{l}\text { Belt interpreted as forming from weathering crusts } \\
\text { developed on Neoproterozoic Mn-bearing rocks. }\end{array}$ \\
\hline $\begin{array}{l}\text { Ezop-Yam- } \\
\text { Alin } \\
\text { (EYA) }\end{array}$ & $\begin{array}{l}\text { W-Mo-Be greisen, stockwork, } \\
\text { and quartz vein (Lednikovy- } \\
\text { Sarmaka); } \\
\text { Sn-W greisen, stockwork, and } \\
\text { quartz vein; } \\
\text { Cassiterite-sulfide-silicate vein } \\
\text { and stockwork; } \\
\text { Porphyry Mo ( } \pm \text { W, Sn, Bi) } \\
\text { (Ippatinskoe, Olgakanskoe, } \\
\text { Shirotnoe) }\end{array}$ & Russia, Far East & $\begin{array}{l}\text { Veins and replacements } \\
\text { related to Khingan- } \\
\text { Okhotsk volcanic-plutonic } \\
\text { belt }\end{array}$ & $\begin{array}{l}\text { Late Cretaceous. Sn granite with } \\
\text { isotoics ages of } 75 \text { to } 100 \mathrm{Ma} \text {. }\end{array}$ & $\begin{array}{l}\text { Belt interpreted as forming during generation of } \\
\text { granitoids along along the Khingan transform } \\
\text { continental-margin arc consisting of the Khingan- } \\
\text { Okhotsk volcanic-plutonic belt that is related to oblique } \\
\text { subduction of ancestral Pacific Ocean Plate. }\end{array}$ \\
\hline $\begin{array}{l}\text { Pilda- } \\
\text { Limuri } \\
(\mathrm{PLL})\end{array}$ & $\begin{array}{l}\text { Sn-W greisen, stockwork, and } \\
\text { quartz vein; } \\
\text { W-Mo-Be greisen, stockwork, } \\
\text { and quartz vein; } \\
\text { Ag-Sb vein (Dyapp); } \\
\text { Polymetallic } \mathrm{Pb}-\mathrm{Zn} \pm \mathrm{Cu}( \pm \mathrm{Ag} \text {, } \\
\text { Au) vein and stockwork } \\
\text { (Uchaminskoye); } \\
\text { Granitoid-related Au vein } \\
\text { (Agnie-Afanasievskoye) }\end{array}$ & Russia, Far East & $\begin{array}{l}\text { Veins, replacements, and } \\
\text { granitoids related to } \\
\text { Khingan-Okhotsk } \\
\text { volcanic-plutonic belt }\end{array}$ & Late Cretaceous & $\begin{array}{l}\text { Belt interpreted as forming during generation of } \\
\text { granitoids along along the Khingan transform } \\
\text { continental-margin consisting of the Khingan-Okhotsk } \\
\text { volcanic-plutonic belt that is related to oblique } \\
\text { subduction of ancestral Pacific Ocean Plate. }\end{array}$ \\
\hline
\end{tabular}




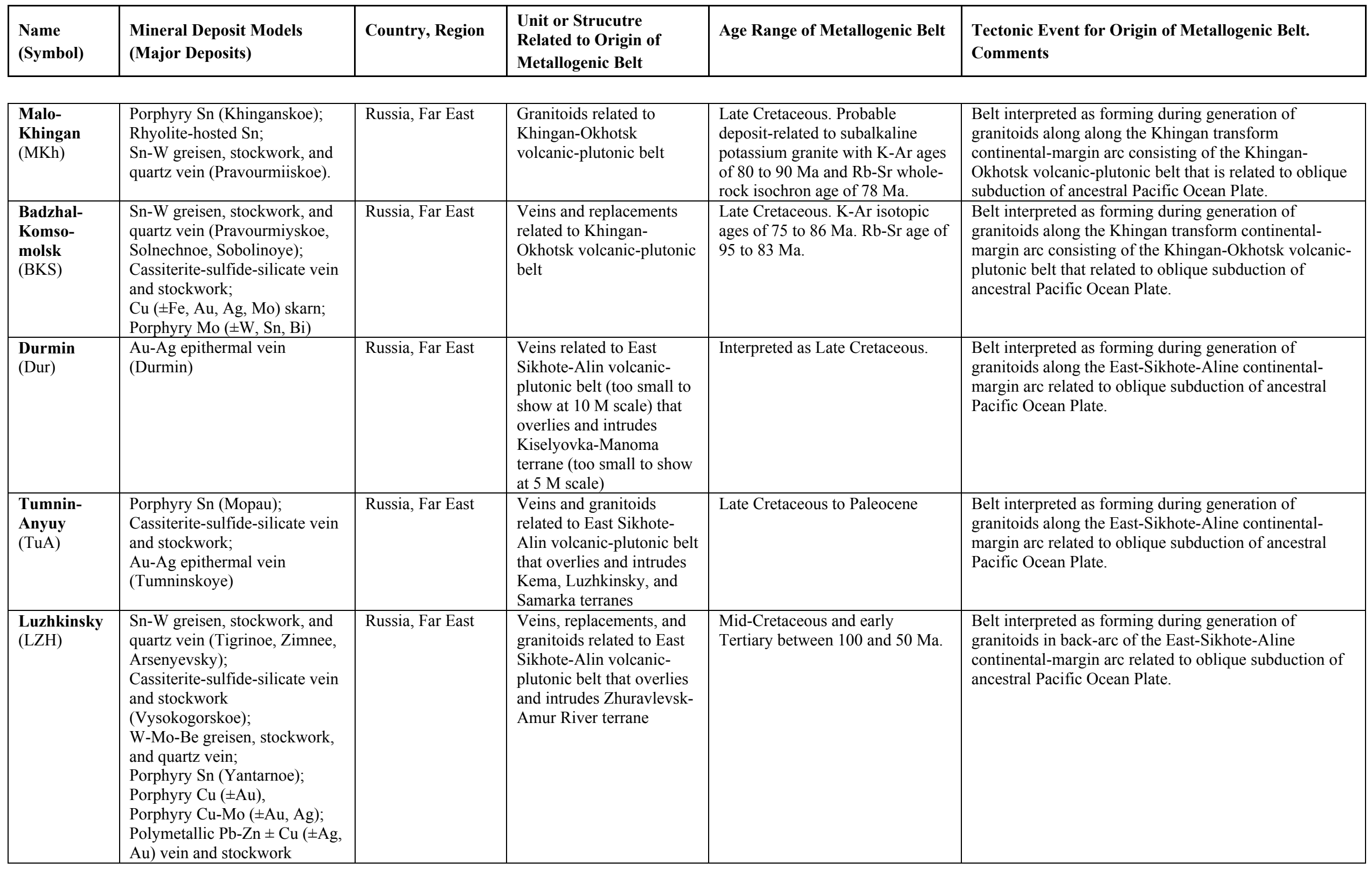




\begin{tabular}{|c|c|c|c|c|c|}
\hline $\begin{array}{l}\text { Name } \\
\text { (Symbol) }\end{array}$ & $\begin{array}{l}\text { Mineral Deposit Models } \\
\text { (Major Deposits) }\end{array}$ & Country, Region & $\begin{array}{l}\text { Unit or Strucutre } \\
\text { Related to Origin of } \\
\text { Metallogenic Belt }\end{array}$ & Age Range of Metallogenic Belt & $\begin{array}{l}\text { Tectonic Event for Origin of Metallogenic Belt. } \\
\text { Comments }\end{array}$ \\
\hline $\begin{array}{l}\text { Sergeevka- } \\
\text { Taukha } \\
\text { (Ser) }\end{array}$ & $\begin{array}{l}\text { Granitoid-related Au vein } \\
\text { (Progress, Askold); } \\
\text { Boron (datolite) skarn } \\
\text { (Dalnegorsk); } \\
\mathrm{Zn}-\mathrm{Pb}( \pm \mathrm{Ag}, \mathrm{Cu}) \text { skarn } \\
\text { (Nikolaevskoe, Partizanskoe); } \\
\text { Polymetallic Pb-Zn } \pm \mathrm{Cu}( \pm \mathrm{Ag}, \\
\text { Au) vein and stockwork; } \\
\text { Porphyry Sn; } \\
\text { Polymetallic }(\mathrm{Pb}, \mathrm{Zn} \pm \mathrm{Cu}, \mathrm{Ba} \text {, } \\
\mathrm{Ag}, \mathrm{Au}) \text { volcanic-hosted } \\
\text { metasomatite } \\
\text { (Krasnogorskoye); } \\
\text { Au-Ag epithermal vein; } \\
\text { Porphyry } \mathrm{Cu}( \pm \mathrm{Au})\end{array}$ & Russia, Far East & $\begin{array}{l}\text { Veins and granitoids } \\
\text { related to East Sikhote- } \\
\text { Alin volcanic-plutonic belt } \\
\text { that overlies and intrudes } \\
\text { Sergeevka, Samarka, and } \\
\text { Taukha terranes }\end{array}$ & $\begin{array}{l}\text { Late Cretaceous and early } \\
\text { Tertiary. K-Ar ages of deposits } \\
\text { range between } 60 \text { and } 80 \text { Ma. }\end{array}$ & $\begin{array}{l}\text { Belt interpreted as forming during generation of } \\
\text { granitoids along the East-Sikhote-Aline continental- } \\
\text { margin arc related to subduction of ancestral Pacific } \\
\text { Ocean Plate. }\end{array}$ \\
\hline \multicolumn{3}{|c|}{$\begin{array}{l}\text { Sambagawa-Chichibu-Shimanto } \\
\text { (SCS) }\end{array}$} & \multicolumn{3}{|c|}{ Started in Late Triassic through Early Jurassic } \\
\hline $\begin{array}{l}\text { Hidaka } \\
\text { (HD) }\end{array}$ & $\begin{array}{l}\text { Cyprus Cu-Zn massive sulfide } \\
\text { (Shimokawa) }\end{array}$ & Japan, Hokkaido & $\begin{array}{l}\text { Stratiform units in } \\
\text { Shimanto accretionary } \\
\text { wedge terrane }\end{array}$ & Middle Cretaceous to Eocene & $\begin{array}{l}\text { Belt interpreted as forming in basalt generated along the } \\
\text { Kula-Pacific ridge. Subsequent structural } \\
\text { incorporatation of host rocks and deposits into an } \\
\text { accretionary wedge. }\end{array}$ \\
\hline $\begin{array}{l}\text { Inner Zone } \\
\text { Southwest } \\
\text { Japan } \\
\text { (ISJ) }\end{array}$ & $\begin{array}{l}\mathrm{Zn}-\mathrm{Pb}( \pm \mathrm{Ag}, \mathrm{Cu}) \text { skarn } \\
\text { (Kamioka Tochibara); } \\
\text { W-Mo-Be greisen, stockwork, } \\
\text { and quartz vein (Otani); } \\
\mathrm{W} \pm \mathrm{Mo} \pm \mathrm{Be} \text { skarn; } \\
\mathrm{Cu}( \pm \mathrm{Fe}, \mathrm{Au}, \mathrm{Ag}, \mathrm{Mo}) \text { skarn } \\
\text { (Bandojima); } \\
\text { Porphyry Mo }( \pm \mathrm{W}, \mathrm{Sn}, \mathrm{Bi}) ; \\
\text { Polymetallic } \mathrm{Pb}-\mathrm{Zn} \pm \mathrm{Cu}( \pm \mathrm{Ag}, \\
\mathrm{Au} \text { ) vein and stockwork } \\
\text { (Ikuno); } \\
\text { Fluorspar vein; } \\
\text { Metamorphic graphite }\end{array}$ & Japan & $\begin{array}{l}\text { Veins and replacements in } \\
\text { Nohi rhyolite volcanic belt } \\
\text { and Hiroshima granitic } \\
\text { belt that overlie and } \\
\text { intrude Hida, Sangun- } \\
\text { Hidagaien-Kurosegawa, } \\
\text { Akiyoshi-Maizuru, Mino- } \\
\text { Tamba-Chichibu terranes } \\
\text { (some too small to show at } \\
10 \mathrm{M} \text { scale). }\end{array}$ & $\begin{array}{l}\text { Cretaceous to Paleogene. } \\
\text { Cretaceous age of deposit-related } \\
\text { granitic rocks in Ryoke and } \\
\text { Sanyo belts are. Paleogene age } \\
\text { mostly for Sanin belt. }\end{array}$ & $\begin{array}{l}\text { Belt interpreted as forming during generation of } \\
\text { granitoids along a East Asia magmatic arc related to } \\
\text { subduction of of Kula and Pacific plates. }\end{array}$ \\
\hline
\end{tabular}




\begin{tabular}{|c|c|c|c|c|c|}
\hline $\begin{array}{l}\text { Name } \\
\text { (Symbol) }\end{array}$ & $\begin{array}{l}\text { Mineral Deposit Models } \\
\text { (Major Deposits) }\end{array}$ & Country, Region & $\begin{array}{l}\text { Unit or Strucutre } \\
\text { Related to Origin of } \\
\text { Metallogenic Belt }\end{array}$ & Age Range of Metallogenic Belt & $\begin{array}{l}\text { Tectonic Event for Origin of Metallogenic Belt. } \\
\text { Comments }\end{array}$ \\
\hline $\begin{array}{l}\text { Gyeongpuk } \\
\text { (GP) }\end{array}$ & $\begin{array}{l}\text { Polymetallic } \mathrm{Pb}-\mathrm{Zn} \pm \mathrm{Cu}( \pm \mathrm{Ag} \text {, } \\
\mathrm{Au} \text { ) vein and stockwork } \\
\text { (Darak, Chilgok); } \\
\text { W-Mo-Be greisen, stockwork, } \\
\text { and quartz vein (Kyeongju); } \\
\text { Sn-W greisen, stockwork, and } \\
\text { quartz vein (Wangpiri); } \\
\text { Fe skarn; } \\
\text { Polymetallic Ni vein } \\
\text { (Samkwang). }\end{array}$ & South Korea & $\begin{array}{l}\text { Veins and replacements } \\
\text { related to Cretaceous } \\
\text { Bulgugsa granite (biotite } \\
\text { granite and granodiorite) } \\
\text { intruding Sino-Korean } \\
\text { Craton - Yeongnam } \\
\text { terrane. }\end{array}$ & $\begin{array}{l}\text { Interpreted as Cenomanian } \\
\text { through Campanian. }\end{array}$ & $\begin{array}{l}\text { Belt interpreted as forming during generation of } \\
\text { Bulgugsa granite during Late Cretaceous to Early } \\
\text { Tertiary Bulgugsa orogeny. Bulgugsa granite consists of } \\
\text { biotite granite, granodiorite, porphyry, and felsic and } \\
\text { quartz-porphyry. Deposits consist of hydrothermal, } \\
\text { fissure-filling sulfide veins in granite gneiss, } \\
\text { granodiorite, and biotite granite. }\end{array}$ \\
\hline $\begin{array}{l}\text { Gyeongnam } \\
(\mathrm{GN})\end{array}$ & $\begin{array}{l}\text { Polymetallic } \mathrm{Pb}-\mathrm{Zn} \pm \mathrm{Cu}( \pm \mathrm{Ag} \text {, } \\
\mathrm{Au}) \text { vein and stockwork; } \\
\text { Polymetallic }(\mathrm{Pb}, \mathrm{Zn} \pm \mathrm{Cu}, \mathrm{Ba}, \\
\mathrm{Ag}, \mathrm{Au}) \text { volcanic-hosted } \\
\text { metasomatite (Gwymyeong, } \\
\text { Mulkum, Kuryong); } \\
\text { Fe skarn Ulsan); } \\
\text { W-Mo-Be greisen, stockwork, } \\
\text { and quartz vein; } \\
\text { Porphyry Mo ( } \pm \mathrm{W}, \mathrm{Sn}, \mathrm{Bi}) ; \\
\mathrm{Cu}-\mathrm{Ag} \text { vein (Goseong, } \\
\text { Tongyoung) } \\
\text { Au in shear zone and quartz } \\
\text { vein (Cheolma) }\end{array}$ & South Korea & $\begin{array}{l}\text { Veins and replacements } \\
\text { related to Cretaceous } \\
\text { Bulgugsa granite (biotite } \\
\text { and feldspar porphyry) } \\
\text { intruding Sino-Korean } \\
\text { Craton - Yeongnam } \\
\text { terrane. }\end{array}$ & $\begin{array}{l}\text { Interpreted as Cenomanian } \\
\text { through Campanian (96-75 Ma). }\end{array}$ & $\begin{array}{l}\text { Belt interpreted as forming during generation of } \\
\text { Bulgugsa granite (biotite granite, granodiorite and } \\
\text { quartz-porphyry) during Late Cretaceous to Early } \\
\text { Tertiary Bulgugsa orogeny. Deposits occur along the } \\
\text { fissures and shear zones. }\end{array}$ \\
\hline $\begin{array}{l}\text { Popigay } \\
\text { (PP) }\end{array}$ & $\begin{array}{l}\text { Impact diamond } \\
\text { (Popigay) }\end{array}$ & $\begin{array}{l}\text { Russia, Northern } \\
\text { Yakutia }\end{array}$ & $\begin{array}{l}\text { Astrobleme formed on } \\
\text { North Asian Craton }\end{array}$ & $\begin{array}{l}\text { Eocene. Tagamite and impact } \\
\text { glasses have }{ }^{40} \mathrm{Ar}-{ }^{39} \mathrm{Ar} \text { isotopic } \\
\text { ages of } 35.7 \mathrm{Ma} \text {. }\end{array}$ & $\begin{array}{l}\text { Belt hosted in Popigay ring structure is interpreted as } \\
\text { resulting from meteoritic impact with formation of } \\
\text { pseudotachylites, high-grade shock metamorphic } \\
\text { minerals, and allogenic breccia. }\end{array}$ \\
\hline \multicolumn{3}{|c|}{ Lower Yana (LY) } & \multicolumn{3}{|c|}{ Started in Cenomanian through Campanian } \\
\hline \multicolumn{3}{|c|}{ Chokhchur-Chekurdakh (CC) } & \multicolumn{3}{|c|}{ Started in Cenomanian through Campanian } \\
\hline \multicolumn{3}{|c|}{ Central Polousnyy (CP) } & \multicolumn{3}{|c|}{ Started in Cenomanian through Campanian } \\
\hline \multicolumn{3}{|c|}{ Eckyuchu-Billyakh (EB) } & \multicolumn{3}{|c|}{ Started in Cenomanian through Campanian } \\
\hline
\end{tabular}




\begin{tabular}{|c|c|c|c|c|c|}
\hline $\begin{array}{l}\text { Name } \\
\text { (Symbol) }\end{array}$ & $\begin{array}{l}\text { Mineral Deposit Models } \\
\text { (Major Deposits) }\end{array}$ & Country, Region & $\begin{array}{l}\text { Unit or Strucutre } \\
\text { Related to Origin of } \\
\text { Metallogenic Belt }\end{array}$ & Age Range of Metallogenic Belt & $\begin{array}{l}\text { Tectonic Event for Origin of Metallogenic Belt. } \\
\text { Comments }\end{array}$ \\
\hline
\end{tabular}

\begin{tabular}{|c|c|c|c|c|c|}
\hline \multicolumn{3}{|l|}{ Taryn (TR) } & \multicolumn{3}{|c|}{ Started in Cenomanian through Campanian } \\
\hline \multicolumn{3}{|l|}{ Selennyakh } & \multicolumn{3}{|c|}{ Started in Cenomanian through Campanian } \\
\hline \multicolumn{3}{|c|}{ Khandyga $(\mathrm{KH})$} & \multicolumn{3}{|c|}{ Started in Cenomanian through Campanian } \\
\hline \multicolumn{3}{|c|}{ South Verkhoyansk } & \multicolumn{3}{|c|}{ Started in Cenomanian through Campanian } \\
\hline \multicolumn{3}{|c|}{ Kukhtuy-Uliya (KU) } & \multicolumn{3}{|c|}{ Started in Cenomanian through Campanian } \\
\hline \multicolumn{3}{|c|}{ Chelasin (CHL) } & \multicolumn{3}{|c|}{ Started in Cenomanian through Campanian } \\
\hline \multicolumn{3}{|c|}{ Preddzhugdzhursky (PRD) } & \multicolumn{3}{|c|}{ Started in Cenomanian through Campanian } \\
\hline $\begin{array}{l}\text { Lower } \\
\text { Amur } \\
\text { (LAM) }\end{array}$ & $\begin{array}{l}\text { Au-Ag epithermal vein } \\
\text { (Mnogovershinnoe); } \\
\text { Epithermal quartz-alunite } \\
\text { (Iskinskoe); } \\
\text { Porphyry } \mathrm{Au} \text {; } \\
\text { Porphyry } \mathrm{Cu}( \pm \mathrm{Au}) \text {; } \\
\text { Sn-W greisen, stockwork, and } \\
\text { quartz vein }\end{array}$ & Russia, Far East & $\begin{array}{l}\text { Veins and granitoids } \\
\text { related to East Sikhote- } \\
\text { Alin volcanic-plutonic belt } \\
\text { that intrudes and overlies } \\
\text { Amur River and } \\
\text { Kiselyovka-Manoma } \\
\text { accretionary-wedge } \\
\text { terranes. }\end{array}$ & $\begin{array}{l}\text { Late Cretaceous and Paleocene. } \\
\mathrm{K}-\mathrm{Ar} \text { isotopic deposit ages of } 49 \\
\text { to } 69 \mathrm{Ma}\end{array}$ & $\begin{array}{l}\text { Belt interpreted as forming during generation of } \\
\text { granitoids along a continental-margin arc related to } \\
\text { subduction of ancestral Pacific Ocean Plate. }\end{array}$ \\
\hline $\begin{array}{l}\text { Kema } \\
(\text { Kem) }\end{array}$ & $\begin{array}{l}\text { Ag-Au epithermal vein } \\
\text { (Glinyanoe, Tayozhnoe 1); } \\
\text { Porphyry Cu-Mo }( \pm \mathrm{Au}, \mathrm{Ag}) \\
\text { Sukhoi Creek; } \\
\text { Porphyry } \mathrm{Cu}( \pm \mathrm{Au}) \\
\text { Verkhnezolotoe); } \\
\text { Porphyry Mo }( \pm \mathrm{W}, \mathrm{Sn}, \mathrm{Bi}) \\
\end{array}$ & Russia, Far East & $\begin{array}{l}\text { Veins related to East } \\
\text { Sikhote-Alin volcanic- } \\
\text { plutonic belt that intrudes } \\
\text { and overlies the Kema } \\
\text { terrane }\end{array}$ & Early Tertiary & $\begin{array}{l}\text { Belt interpreted as forming during generation of } \\
\text { granitoids along a continental-margin arc related to } \\
\text { subduction of ancestral Pacific Ocean Plate. }\end{array}$ \\
\hline \multicolumn{3}{|c|}{ Luzhkinsky (LZH) } & \multicolumn{3}{|c|}{ Started in Cenomanian through Campanian } \\
\hline \multicolumn{3}{|c|}{ Hidaka (HD) } & \multicolumn{3}{|c|}{ Started in Cenomanian through Campanian } \\
\hline \multicolumn{3}{|c|}{ Inner Zone Southwest Japan (ISJ) } & \multicolumn{3}{|c|}{ Started in Cenomanian through Campanian } \\
\hline \multicolumn{6}{|c|}{ MIOCENE THROUGH QUATERNARY (24 to 0 Ma) } \\
\hline $\begin{array}{l}\text { Northeast } \\
\text { Hokkaido } \\
\text { (NEH) }\end{array}$ & $\begin{array}{l}\text { Au-Ag epithermal vein } \\
\text { (Konomai); } \\
\text { Volcanic-hosted } \mathrm{Hg} \text { (Itomuka); } \\
\text { Hg-Sb-W vein and stockwork } \\
\text { (Ryushoden); } \\
\text { Clastic sediment-hosted } \mathrm{Hg} \pm \mathrm{Sb}\end{array}$ & Japan, Hokkaido & $\begin{array}{l}\text { Veins and replacements in } \\
\text { Quaternary Japan volcanic } \\
\text { belt and Neogene Japan } \\
\text { sedimentary basin that } \\
\text { overlies and intrudes } \\
\text { Hidaka zone of the } \\
\text { Shimanto accretionarry } \\
\text { wedge terrane. }\end{array}$ & $\begin{array}{l}\text { Miocene to Quaternary. Two ages } \\
\text { of deposits: early stage ( } 14.4 \text { to } \\
11.2 \mathrm{Ma} \text { ); and late stage }(8.1 \text { to } \\
0.3 \mathrm{Ma}) \text {. }\end{array}$ & $\begin{array}{l}\text { Belt interpreted as forming along an island arc related to } \\
\text { subduction of the Pacific Plate beneath eastern } \\
\text { Hokkaido Island. }\end{array}$ \\
\hline
\end{tabular}




\begin{tabular}{|c|c|c|c|c|c|}
\hline $\begin{array}{l}\text { Name } \\
\text { (Symbol) }\end{array}$ & $\begin{array}{l}\text { Mineral Deposit Models } \\
\text { (Major Deposits) }\end{array}$ & Country, Region & $\begin{array}{l}\text { Unit or Strucutre } \\
\text { Related to Origin of } \\
\text { Metallogenic Belt }\end{array}$ & Age Range of Metallogenic Belt & $\begin{array}{l}\text { Tectonic Event for Origin of Metallogenic Belt. } \\
\text { Comments }\end{array}$ \\
\hline $\begin{array}{l}\text { Northeast } \\
\text { Japan } \\
\text { (NEJ) }\end{array}$ & $\begin{array}{l}\text { Volcanogenic } \mathrm{Zn}-\mathrm{Pb}-\mathrm{Cu} \\
\text { massive sulfide (Kuroko, Altai } \\
\text { types) (Kosaka, Shakanai); } \\
\text { Au-Ag epithermal vein } \\
\text { (Sado, Hosokura, Toyoha); } \\
\text { Polymetallic (Pb, Zn } \pm \mathrm{Cu}, \mathrm{Ba} \text {, } \\
\mathrm{Ag}, \mathrm{Au}) \text { volcanic-hosted } \\
\text { metasomatite; } \\
\text { Sulfur-sulfide (S, FeS } 2 \text { ) } \\
\text { (Horobetsu); } \\
\text { Polymetallic } \mathrm{Pb}-\mathrm{Zn} \pm \mathrm{Cu}( \pm \mathrm{Ag} \text {, } \\
\text { Au) vein and stockwork } \\
\text { (Ashio); } \\
\text { Mn vein; } \\
\text { Volcanogenic-sedimentary Mn } \\
\text { (Kinjo); } \\
\text { Chemical-sedimentary Fe-Mn; } \\
\text { Limonite from spring water } \\
\text { (Gumma) }\end{array}$ & Japan & $\begin{array}{l}\text { Layers and veins in } \\
\text { Quaternary Japan volcanic } \\
\text { belt and Neogene Japan } \\
\text { sedimentary basin that } \\
\text { overlie and intrude } \\
\text { Hiroshima granitic } \\
\text { plutonic belt, and Mino- } \\
\text { Tamba-Chichibu and } \\
\text { South Kitakami terranes. }\end{array}$ & $\begin{array}{l}\text { Miocene to Quaternary. Many } \\
\text { Kuroko-type deposits were } \\
\text { formed in middle Miocene, at } \\
\text { about } 13 \text { Ma. K-Ar ages of vein } \\
\text { deposits suggest two stages of } \\
\text { formation: early stage ( } 15 \text { to } 10 \\
\text { Ma), and late stage ( } 8 \text { to } 2 \mathrm{Ma} \text { ). } \\
\text { Sulfur-sulfide (S, FeS2) and } \\
\text { limonite deposits formed on } \\
\text { Quaternary volcanoes. }\end{array}$ & $\begin{array}{l}\text { Volcanogenic massive sulfide deposits interpreted as } \\
\text { forming in back-arc region of an island arc related to } \\
\text { subduction of the Pacific Plate beneath eastern } \\
\text { Hokkaido Island. Au-Ag epithermal vein deposits } \\
\text { interpreted as forming along axial part of an island arc } \\
\text { volcanism. Sulfur-sulfide and limonite deposits formed } \\
\text { in active island arc. Island arc magmatism related to } \\
\text { subduction of Pacific Plate. }\end{array}$ \\
\hline $\begin{array}{l}\text { Hokuriku- } \\
\text { Sanin } \\
\text { (Hok) }\end{array}$ & $\begin{array}{l}\text { Au-Ag epithermal vein } \\
\text { (Omori); } \\
\text { Polymetallic } \mathrm{Pb}-\mathrm{Zn} \pm \mathrm{Cu}( \pm \mathrm{Ag} \text {, } \\
\text { Au) vein and stockwork } \\
\text { (Taishu); } \\
\text { Ag-Sb vein; } \\
\text { Clastic-sediment-hosted } \mathrm{U}\end{array}$ & Japan & $\begin{array}{l}\text { Veins and replacements } \\
\text { related to Neogene Japan } \\
\text { sedimentary basin that } \\
\text { overlies and intrudes } \\
\text { Hiroshima granitic } \\
\text { plutonic belt, and } \\
\text { Akiyoshi-Maizuru and } \\
\text { Mino-Tamba-Chichibu } \\
\text { terranes. }\end{array}$ & Miocene to Pleistocene & $\begin{array}{l}\text { Belt interpreted as forming along an island arc during } \\
\text { back-arc rifting or axial part of an island arc that was } \\
\text { related to subduction of Philippine Sea Plate. }\end{array}$ \\
\hline
\end{tabular}




\begin{tabular}{|c|c|c|c|c|c|}
\hline $\begin{array}{l}\text { Name } \\
\text { (Symbol) }\end{array}$ & $\begin{array}{l}\text { Mineral Deposit Models } \\
\text { (Major Deposits) }\end{array}$ & Country, Region & $\begin{array}{l}\text { Unit or Strucutre } \\
\text { Related to Origin of } \\
\text { Metallogenic Belt }\end{array}$ & Age Range of Metallogenic Belt & $\begin{array}{l}\text { Tectonic Event for Origin of Metallogenic Belt. } \\
\text { Comments }\end{array}$ \\
\hline $\begin{array}{l}\text { Kyushu } \\
\text { (Kus) }\end{array}$ & $\begin{array}{l}\text { Au-Ag epithermal vein } \\
\text { (Hishikari, Kushikino, Taio) }\end{array}$ & Japan & $\begin{array}{l}\text { Veins and replacements } \\
\text { related to Quaternary } \\
\text { Japan volcanic belt and } \\
\text { Neogene Japan } \\
\text { sedimentary basin that } \\
\text { overlie and intrude } \\
\text { Akiyoshi-Maizuru, } \\
\text { Shimanto, and Mino- } \\
\text { Tamba-Chichibu terranes. }\end{array}$ & Pliocene to Quaternary & $\begin{array}{l}\text { Belt is interpreted as forming during hydrothermal } \\
\text { activitiy along a Pliocene and Quaternary island arc } \\
\text { during back-arc rifting or the axial part of an island arc } \\
\text { that was related to subduction of Philippine Sea Plate. }\end{array}$ \\
\hline
\end{tabular}

